Portland State University

PDXScholar

Summer 8-4-2014

\title{
Testing the Ecological and Physiological Factors Influencing Reproductive Success in Mosses
}

Erin Elizabeth Shortlidge

Portland State University

Follow this and additional works at: https://pdxscholar.library.pdx.edu/open_access_etds

Part of the Other Plant Sciences Commons, and the Plant Biology Commons Let us know how access to this document benefits you.

\section{Recommended Citation}

Shortlidge, Erin Elizabeth, "Testing the Ecological and Physiological Factors Influencing Reproductive Success in Mosses" (2014). Dissertations and Theses. Paper 1951.

https://doi.org/10.15760/etd.1950

This Dissertation is brought to you for free and open access. It has been accepted for inclusion in Dissertations and Theses by an authorized administrator of PDXScholar. Please contact us if we can make this document more accessible: pdxscholar@pdx.edu. 
Testing the Ecological and Physiological Factors Influencing

Reproductive Success in Mosses

by

Erin Elizabeth Shortlidge

A dissertation submitted in partial fulfillment of the requirements for the degree of

Doctor of Philosophy in

Biology

\begin{abstract}
Dissertation Committee:
Sarah Eppley, Chair

Daniel Ballhorn

Catherine de Rivera

Jason Podrabsky

Todd Rosenstiel
\end{abstract}

Portland State University

2014 
(C) 2014 Erin Elizabeth Shortlidge 


\begin{abstract}
As non-vascular, early land plants with an aquatic ancestry, mosses do not regulate internal water conditions separate from that of their environment and as a result, evolved mechanisms to survive in a terrestrial world out of water. Yet, there is a widely accepted dogma that moss reproductive success is solely dependent on rainfall events carrying swimming, bi-flagellate sperm across the landscape to reproductively mature and receptive female mosses but this classic view of moss reproduction may be too simplistic. In this dissertation I test the assumptions of reproductive limitation in mosses and present novel findings in a basal, yet understudied terrestrial plant mating system.
\end{abstract}

I find evidence of environmental desiccation tolerance in moss sperm, thus offering the potential for stress-tolerant gametes on the landscape possibly suited for various transport vectors, reminiscent of a pollen grain. To investigate the broad evolutionary implications of this newfound complexity in moss sexual reproduction, I tested classic tenets of plantpollinator theory on the ancient mosses and their ubiquitous microarthropod inhabitants. Experimental results show that mosses and microarthropods are engaged in a "pollination-like" syndrome guided by sex-specific volatile cues that differentially affect microarthropod behavior. These data indicate an existing complex mutualistic relationship and provides new evidence of sexually dimorphic investment by male and female mosses into reproductive 
success. Further, these data put forth the idea that female mosses challenge an inherent mate limitation by investing into reproductive assurance via maintaining a relationship with microarthropods.

Experimental work further confirms a role for invertebrates in moss reproductive success and tests mutualism theory through ongoing experiments. Such experiments include an assessment of moss genetic diversity, paternity, and male fitness traits as it relates to mosses with or without the presence of microarthropods, thereby testing for fitness benefits gained by mosses possibly engaged in a transport mutualism with microarthropods.

I further tested mutualism, community ecology and moss sexual reproduction concepts in extreme geothermal moss populations living at the edges of inhabitable Earth, and results show that even geothermal moss canopies are diverse and host differential and abundant life. In a first field test of mutualism I found that although extreme heat stress may constrain sexual reproduction in mosses, a correlation between within-population moss genetic diversity and microarthropod abundances exists. To further examine mosses in extreme environments, and how these environments may constrain sexual reproductive success, I evaluated the effects of simulated warming on Antarctic moss physiology and reproductive biology. Data indicates that simulated warming relieves mosses of physiological stress, and results in a greater investment into primary productivity and sexual reproduction. These 
data support the hypothesis that with less stress, sexual reproduction is increased. Mosses are an ideal system by which to understand organisms that exist in environments ranging from the mesic to the extreme, in the laboratory and the field and even in the classroom, where the small functioning ecosystem of mosses can be used for discovery-based biology education as described in the Mosscosms curriculum.

Overall, this work contributes significantly to the field of bryophyte and plant biology by revealing novel insights into the biotic and abiotic factors influencing sexual reproduction in mosses. 
The fairest thing we can experience is the mysterious. It is the fundamental emotion which stands at the cradle of true art and true science. He who knows it not and can no longer wonder, no longer feel amazement, is as good as dead, a snuffed-out candle.

Albert Einstein, The World as I See it (1931) 


\section{Acknowledgements}

First and foremost I would like to acknowledge the never-ending love from my mom - no matter what my diverse endeavors have been, you have supported them. I would like to thank my cat Emmett who has sat on my keyboard and/or halfway over my right arm for at least half of the words written in this dissertation.

I thank my advisor Sarah Eppley for her ability to always see the path forward although it was not always clear to me. You have believed in my ability to conquer this $\mathrm{PhD}$ and I deeply appreciate your confidence and continuous generosity. I clearly would not be here, writing this without you. I thank Todd Rosenstiel for invaluable insights and endless encouragement as well as Suzanne Estes and Jason Podrabsky for general scientific, moral and logistical support, even at a moment's notice. I also would like to thank Catherine de Rivera for years of support and guidance, and Daniel Ballhorn for your insight.

I am grateful for the opportunity to participate in the GK-12 program as it allowed me work with incredible students and helped me to see what aspects of my scientific endeavors actually matter the most. The fieldwork and life I experienced in Antarctica with Angelica Casanova-Katny are incomparable, and to everyone that I have been lucky enough to collaborate with - your talents are definitely not lost on me. 
I thank my labmates both past and present who have played a role of support and understanding that cannot be replicated outside of our insular world, and the undergraduates and post-bacs who worked diligently and sometimes tirelessly to help me with data collection.

I would like to acknowledge the funding sources that have allowed me to pursue this work and have enabled the experiences that I have gained along the way.

I also would like to thank my constant work companions: A few particular albums listened to on constant repeat while experiments were setup, maintained, and data collected, as well as most of Oregon Public Broadcasting's programming. Also, thank you to Besaw's.

And finally, I owe so much to my family and friends - both in Portland and far away, you know who you are, and each of you are very much appreciated and loved. I may not have made it through this (or any) phase of my life without you being there to tirelessly pick up the line when the phone calls came in. Thank you. 


\section{Table of Contents}

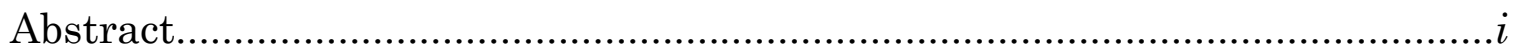

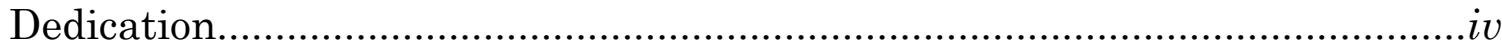

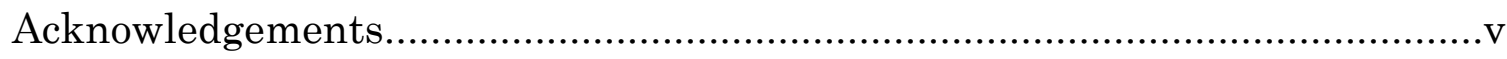

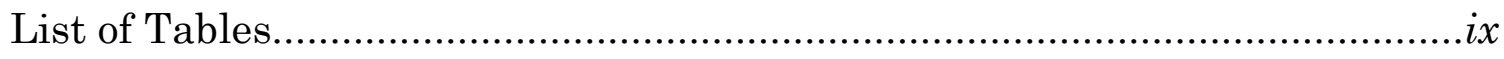

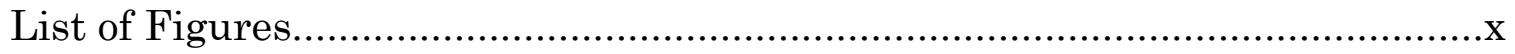

\section{Chapter 1}

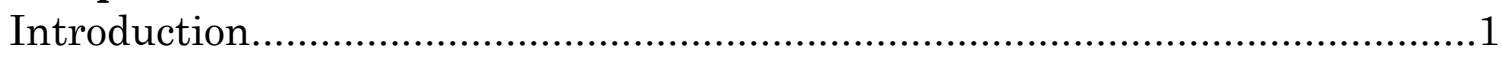

\section{Chapter 2}

Tolerance to environmental desiccation in moss sperm ..................................6

\section{Chapter 3}

Sex-specific volatile compounds influence microarthropod-mediated

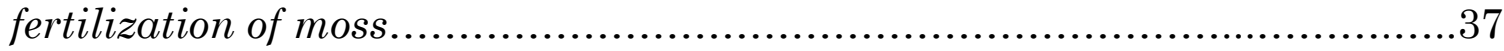

\section{Chapter 4}

Community ecology of geothermal mosses: what drives diversity at temperature extremes?.

\section{Chapter 5}

Warming reduces the physiological barrier to reproductive success in Antarctic mosses.

\section{Chapter 6}

Mosscosms: A classroom activity to encourage ecosystem-level thinking.

\section{Chapter 7}

Discussion.

References 


\section{Appendices}

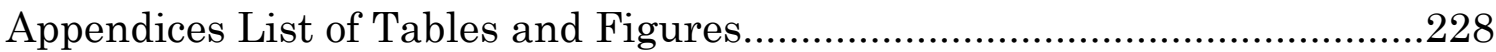

\section{Appendix A}

Supplemental materials to Chapter 3: Sex-specific volatile compounds

influence microarthropod-mediated fertilization of moss.

\section{Appendix B}

Testing biotic and abiotic influences on the reproductive biology of the monoicous moss, Physcomitrella patens.........................................265

\section{Appendix C}

Testing mutualism in two of Earth's most ancient lineages: the mosses and microarthropods..............................................................282

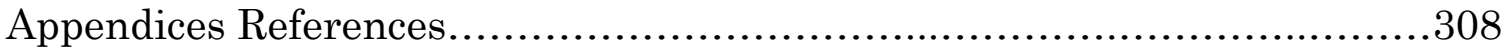




\section{List of Tables}

Table 2.1 Tolerance to desiccation in moss sperm...............................32

Table 4.1 Results of 2-way ANOVA between and among geothermal

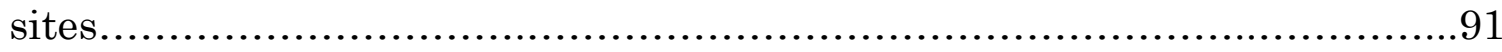

Table 4.2 Results of 2-way ANOVA between and among invertebrate collection method results and study site.......................................91

Table 4.3 Values and ANOVA statistics for variables by LVNP site...........92 Table 4.4. Principal Component Scores from multivariate community analysis on correlation matrix.....................................................

Table 5.1 Gametophyte numbers and relative percent of $P$. alpinum cores

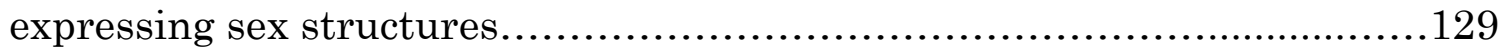

Table 6.1 Student TOSRA Survey Results......................................158 


\section{List of Figures}

2.1 Effects of dry-down rate on sperm cell recovery from environmental

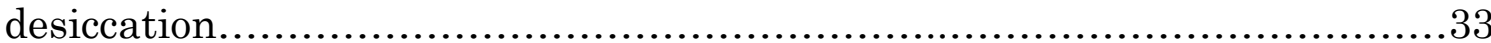

2.2 Exogenous sucrose aids sperm cells in recovery from environmental desiccation events

2.3 Variation among individuals in sperm cell recovery from environmental desiccation.

2.4 Effects of time and dehydration treatments on sperm cell integrity in three species of mosses.

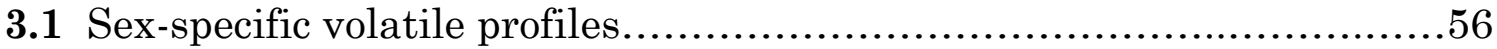

3.2 Significant differences in volatile composition............................57

3.3 Springtails prefer female moss.............................................58

3.4 Springtails enhance fertilization in moss microcosms.....................59

4.1 Abiotic profiles of five geothermal sites...................................95

4.2 C. purpureus temperatures along a vertical gradient........................96

4.3 Invertebrate community group diversity and abundance..................97

4.4 Total invertebrates collected by method at each of five sites...............98

4.5 C. purpureus within population genetic variation by site...................99

4.6 Principal component analysis of LVNP geothermal sites..................100

5.1 Temperatures in control and OTC plots in January, 2014 ..................130

5.2 Relative humidity at moss canopy level....................................131

5.3 Community assemblages by plot........................................ 132

5.4 Species abundances by treatment.........................................133

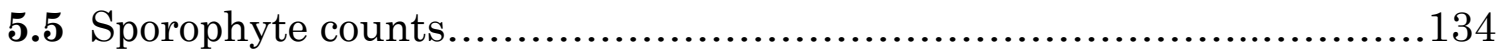

5.6 In situ physiological status of $P$. alpinum canopies.........................135 
5.7 Physiological status of $P$. alpinum by treatment............................136

5.8 Composition of $P$. alpinum cores............................................137

6.1 Student-generated springtail food choice results..............................160

6.2 TOSRA survey results..................................................161

6.3 Student responses to written questions.....................................162 


\section{Chapter 1}

\section{Introduction}

Mosses make up the largest phylum (Bryophyta) of non-vascular bryophytes, and is comprised of 10,000-15,000 individual moss species (Shaw \& Goffinet, 2009, Vanderpoorten \& Goffinet, 2009, Crosby \& Garden, 2000). The most basal extant relatives of modern bryophytes are some of the Earth's first terrestrial plants, dating back to the mid-late Ordovician, about 450 Mya (Kenrick \& Crane, 1997). A derived trait of all subsequent land plants is that they are embryophytes, meaning that the female egg is stationary and once fertilized, develops into an ovary that is nourished by the female to maturation (Island \& Arctic, 2001, Mishler \& Churchill, 1985), thus anchoring the plants and requiring the development of dispersal mechanisms. The mechanisms they evolved proved successful as mosses are now found across the globe from pole to pole (Shaw \& Goffinet, 2000).

In order to thrive in such wide-ranging environments mosses must be, and are, incredibly stress tolerant relative to many other taxa (During, 1979, Oliver et al., 2000) One of mosses' most necessary adaptations to terrestrial life out of water was the need to tolerate water stress (Oliver et al., 2005). Mosses do not have cuticles or physical barriers that keep them from dryingout, but instead are poikilohydric organisms. This means that they do not maintain an internal water balance separate from ambient conditions, 
therefore even mosses living in mesic environments maintain some degree of desiccation tolerance (Oliver et al., 2000, Proctor et al., 2007b). Mosses can essentially halt all metabolic processes upon experiencing desiccation stress, only to quickly resume normal function and metabolism upon rehydration (Oliver et al., 2005, Proctor et al., 2007). The majority of environments do not maintain ambient humidity conditions that would be ideal for moss, yet across the globe mosses are regularly surviving, growing and reproducing.

Like most other eukaryotes, mosses require the fusion of a sperm and an egg for sexual reproduction to occur. Some mosses have both male and female sex organs on the same gametophyte (monoicous) and others maintain separate sexes (dioicous), with chromosomally determined sex (McDaniel et al., 2013b). Mosses have biflagellate sperm that must travel ex planta to a receptive, mature female moss of the same species for fertilization success. This practice is relatively simplified when a monoicous moss has male and female organs on the same individual gametophyte. Given that these organisms are potentially regularly experiencing environmental stress, such as desiccation, this should theoretically limit the evolution of dioicous moss species and select for monoicy, but this is not the case. In fact, $\sim 60 \%$ of all moss species are dioicous (Wyatt \& Anderson, 1984), and there has been recurrent evolution towards dioicy in mosses (identified over 90 times) solidifying the prevalence of a dioicous mating system (McDaniel et al., 2013a). These data imply that selection is acting on the maintenance of 
separate sexes in the majority of mosses worldwide, but the "why" is still unresolved.

My thesis work is comprised of cross-disciplinary integrated approaches testing the biotic and abiotic factors that influence sexual reproduction and the overall success of common dioicous moss species. I test this question in mesic environments of the Pacific Northwest using laboratory, greenhouse-based, and outdoor microcosms, as well as in the field where mosses occur at environmental extremes on the front lines of abiotic stress and global climate change.

- In Chapter 2 I describe ways in which I have tested the physiology of moss sperm, in order to understand how the swimming sperm may survive their journey across the landscape.

- In Chapter 3 I use tenets of angiosperm plant-pollinator theory to guide comprehensive, ecologically relevant tests of the relationships between environmental factors, moss-canopy microarthropods and moss physiology on the fertilization success of a common dioicous moss species, Ceratodon purpureus.

- In Chapter 4 I investigate the relative influence of the biotic and abiotic factors impacting geothermal moss-community function and diversity along an environmental gradient.

- In Chapter 5 I assess the effects of simulated climate-warming on moss and lichen-dominated communities of the western Antarctic 
Peninsula, and on the physiology and reproductive efforts of a common cold-adapted dioicous moss, Polytrichastrum alpinum

- Chapter 6 I describe how to use the moss-microarthropod microcosm system as an active-learning model for teaching ecological concepts at the middle school level

- Chapter 7 I provide a discussion of the work presented

- In the Appendices I present supplementary data on C. purpureus volatile organic compounds and canopy metrics, implications for microarthropods on the reproduction of a monoicous moss species, and finally, I discuss ongoing work and intriguing preliminary data testing the implications of a mutualistic plant-pollinator-like relationship between canopy microarthropods and C. purpureus.

My dissertation work adds significantly to the existing body of knowledge on the often over-looked and over-simplified reproductive ecology of mosses by testing and investigating the influencing factors, as well as strengthens the argument for the importance of understanding the biology of these plants as a functioning, complex system. In Chapters 2-5, as outlined above, I describe the work I have completed towards this dissertation, in Chapter 6 I discuss how I used my dissertation research topic as a successful education-outreach, active learning model in a public middle school, generating original curricula that yielded novel scientific 
results, and Chapter 7 presents a discussion outlining the broader implications of this work and incorporates directions for future study. 


\title{
Chapter 2
}

\section{Tolerance to environmental desiccation in moss sperm}

\begin{abstract}
Sexual reproduction in mosses requires that sperm be released freely into the environment before finding and fertilizing a receptive female. After release from the male plant, moss sperm may experience a range of abiotic stresses; however, little data are available examining stress tolerance of moss sperm and whether there is genetic variation for stress tolerance in this important life stage. Here, we investigated the effects of environmental desiccation and recovery on the sperm cells of three moss species (Bryum argenteum, Campylopus introflexus, and Ceratodon purpureus). We found that a fraction of sperm cells were tolerant to environmental desiccation for extended periods (days) and that tolerance did not vary among species. We found that this tolerance occurs irrespective of ambient dehydration conditions, and that the addition of sucrose during dry-down improved cell recovery. Although we observed no interspecific variation, significant variation among individuals within species in sperm cell tolerance to environmental desiccation was observed, suggesting selection could potentially act on this basic reproductive trait. The observation of desiccation tolerant sperm in multiple moss species has important implications for understanding bryophyte reproduction, suggesting the presence of a
\end{abstract}


significant, uncharacterized complexity in the ecology of moss mating systems.

Key words: Ceratodon purpureus, bryophyte, sexual reproduction, sperm, stress tolerance 


\section{Introduction}

The majority of eukaryotic male organisms produce gametes in the form of sperm, which are generally motile and must travel before arriving at a receptive female. Within eukaryotic organisms, sperm may experience a diversity of situations before reaching the female egg, including free release into the environment (Franzen 1956, Jamieson \& Rouse, 1989, Lord \& Russell, 2002, Vanderpoorten \& Goffinet, 2009). Land plants require sperm to move some distance, ex planta, to fertilize the female egg and form a zygote, but they do so via diverse pathways. For the majority of tracheophytes, including gymnosperms and angiosperms, male sperm are contained during transport by pollen grains, which are carried by wind or insect across the terrestrial landscape to compatible female sex structures. Upon germination, the pollen grain grows a protective pollen tube that delivers the sperm internally to the egg (Brewbaker \& Kwack, 1963, Lord \& Russell, 2002, Taylor \& Hepler, 1997) although in some gymnosperms (e.g., cycads) flagellated sperm travel internally to fertilize the egg after pollination. Alternately, the non-seed tracheophytes including Pterophytes (ferns) and Bryophytes (mosses, liverworts, hornworts) release sperm onto the landscape for water dispersal (Garbary et al., 1993, Grout, 1933, Vanderpoorten \& Goffinet, 2009). The journey of these sperm is unique as the sperm are neither released into a body of water nor protected by a pollen grain, but 
instead are freed onto the terrestrial landscape in an often-ephemeral film of water (Muggoch \& Walton, 1942, Paolillo, 1981)

Bryophyta, or mosses, are the most species-rich of the three phyla of bryophytes, and the most widely distributed of all seedless tracheophytes (Shaw \& Goffinet, 2000). Male reproductive organs (antheridia) of sexually reproducing mosses dehisce lipid-rich sperm masses that contain numerous bi-flagellated sperm for dispersal into the environment (Paolillo, 1981). However, little information is known about the fate and journey of the moss sperm, a presumably sensitive life stage, once they are freed from the sperm mass to disperse and arrive at a receptive female. The common conjecture has been that the sperm of mosses are dispersed via mass flow of water or by water-droplets from splash cups, and as a result dispersal distances are relatively small (Clayton-Greene et al., 1977, Longton \& Greene, 1979, Wyatt, 1977). This limited view of sperm dispersal comes under scrutiny with further recent research indicating that moss sperm may disperse an order of magnitude farther than previously believed (Bisang et al., 2004, Van der Velde et al., 2001b). It has been long speculated that microarthropods are involved in sperm dispersal, as they are regular constituents of moss canopies worldwide (Gerson, 1969, Gibson \& Miller-Brown, 1927). This idea was tested and supported in laboratory experiments conducted by Cronberg et. al. (2006), using the moss Bryum argenteum in conjunction with added springtails and mites, which are both common inhabitants of moss canopies 
(Lindo \& Winchester, 2006). These data strongly suggest that a more comprehensive perception of the ecology of moss mating systems is necessary, particularly as over $60 \%$ of mosses have separate sexes (Wyatt \& Anderson, 1984) rendering potentially significant distances between male and female mosses a distinct possibility.

Similarly, our understanding of the relationship between sexual reproduction in mosses and environmental stress is limited (Convey \& Smith, 1993, Stark et al., 2000) despite the fact that mosses are globally prevalent and well-known for their ability to tolerate environmental stress (Clarke et al., 2008, Lovelock et al., 1995, Meyer \& Santarius, 1998, Seel et al., 1992b). The majority of research previously conduced on moss sperm focused on sperm architecture and the composition of the sperm mass (Bernhard \& Renzaglia, 1995, Paolillo, 1977, Paolillo, 1979, Paolillo, 1981, Renzaglia et al., 1995); the mechanism of initial sperm release (Paolillo, 1975); and the behavior of the released sperm mass upon encountering the air-water interface (Muggoch \& Walton, 1942, Paolillo, 1981). Working forward from these studies in combination with what we know about the daily stresses of many mosses, it seems pertinent to investigate the effects of stress on the reproductive cycle of mosses. To our knowledge, only one previous study examined the impacts of environmental stress on moss sperm (Rosenstiel \& Eppley, 2009). This study found that sperm cells of the geo-thermal moss Pohlia nutans exhibit remarkable thermo-tolerance, with cells maintaining 
integrity at temperatures of $60^{\circ} \mathrm{C}$. Whether this property is unique to geothermal populations of $P$. nutans, or more broadly observed in moss sperm, remains unknown. If moss sperm disperse over long distances and these distances include potentially stressful environments of extreme temperatures, sun-exposed canopies, and microarthropod bodies, selection may increase sperm tolerance to those environments, thus enhancing reproductive success of males with stress tolerant sperm.

All organisms require water for normal metabolic activity (Hochachka \& Somero, 2002) yet taxa from a variety of independent lineages are able to survive extreme dehydration (Giard, 1894). In plants, this ability is widespread at reproductive stages (e.g., pollen, seeds, spores), but less common in vegetative stages (primarily found in algae, mosses and lichen; (Alpert, 2000)). Surviving the loss of cellular water requires having a suite of stress response traits that are associated with preventing oxidative damage, stabilizing macromolecules, and maintaining membrane integrity (Hochachka \& Somero, 2002, Hoekstra et al., 2001). Plants vary in the degree to which they can undergo these responses, in whether they rely on constitutive or induced protection, and if they can repair damage upon recovery (Alpert, 2000). Several characteristics of plant response to extreme dehydration are common among taxa, including cellular recovery from stress being dependent on the rate of water loss (e.g., Oliver et al., 1997, Schonbek \& Bewley, 1981), and the ability to accumulate sugars during the latter 
stages of dehydration (e.g., Bewley, 1979, Hoekstra \& Vanroekel, 1988, Koster \& Leopold, 1988, Sun et al., 1994). Of the possible and probable abiotic stresses encountered by moss, water stress could be argued the most physiologically and ecologically relevant to the micro-world of mosses as they are poikilohydric plants (Proctor et al., 2007) and thereby are unable to regulate internal water relations separate from that of their surrounding environment. In some habitats, mosses may experience dehydration events that range from seasonal, to monthly, to daily (Alpert, 2000, Oliver et al., 2005). Most mosses are believed to possess some degree of desiccation tolerance (Proctor \& Pence, 2002), and like other organisms that tolerate extreme water stress, can dehydrate to exceedingly low water contents (5$10 \%$ of their dry weight) and regain physiological function upon rehydration. Gametophytic tolerance to desiccation in mosses has been well studied (Barker et al., 2005, Bewley, 1973, Bewley, 1979, Dilks \& Proctor, 1979, Proctor et al., 2007), and mosses are quickly emerging as an important model system for understanding the molecular and cellular basis of desiccation tolerance (Cove et al., 2006, Cuming et al., 2007, Wood \& Oliver, 2004). Nonetheless, only a few studies have focused on desiccation tolerance in moss reproductive structures, including on sporophytes and asexual propagules (Oliver et al., 2000b, Proctor \& Smirnoff, 2000, Rowntree et al., 2007, Stark, 2002). A remaining question is whether desiccation tolerance is also present in the sperm cells of mosses. Desiccation tolerance in moss sperm would 
significantly challenge the assumption that moss mating systems are dictated by available free water.

The objective of this study was to investigate how dehydrationrehydration events impact sperm cell integrity in three moss species. We chose three cosmopolitan species with separate sexes that all exhibit desiccation tolerance in their gametophyte phase (Proctor et al., 2007; Wood \& Oliver, 2007): Bryum argenteum (Bryaceae), Campylopus introflexus (Dicranaceae), and Ceratodon purpureus (Ditrichaceae). Specifically, we examined: 1) whether environmental desiccation tolerance in moss sperm is influenced by the rate at which cells dehydrate, 2) the impact of exogenous sugars (sucrose) on environmental desiccation tolerance of moss sperm, and 3) the potential for individual or species-level variation in moss sperm tolerance to environmental desiccation.

\section{Materials and Methods}

\section{Study species and selection of sexual structures}

Three moss species were used for experiments described in this study.

Ceratodon purpureus (Hedw.) Brid. and Bryum argenteum (Hedw) are nearly cosmopolitan species, with separate sexes (Shaw \& Beer, 1999, Smith \& Convey, 2002a). Campylopus introflexus Brid. also has separate sexes and is native to the southern hemisphere, but was relatively recently introduced to North America (Frahm, 1980, Gradstein \& Sipman, 1978). All three of these 
moss species have experimentally been found to be tolerant to desiccation events (Equihua \& Usher, 1993, Nabe et al., 2007, Proctor, 2001, Robinson et al., 2000).

Plants were collected in 2009 for this study from various locations. Three C. purpureus populations were collected in the Portland, Oregon greater metropolitan area from: 1) northeast Portland, 2) the Portland State University campus, and 3) North Plains, Oregon, with populations a minimum of $5.8 \mathrm{~km}$ apart. The $B$. argenteum plants were from 1) southern Arizona, 2) northeast Portland, 3) southwest Portland, and 4) the University of Kentucky campus. C. introflexus plants were collected from 1) Lassen Volcanic National Park (LVNP); (a subset of which were stored dry for transport and longevity, until we were successful at growing $C$. introflexus to sexual maturity in the greenhouse), and 2) the central Oregon coast. Plants were grown in $6.4-\mathrm{x}-6.4 \mathrm{~cm}$ pots on a substrate of $2: 1$ propagation grade sand and peat moss. Individual plants were field collected and then grown either from single spore or from gametophyte cuttings. Mosses grown from spore were maintained in Adaptis 1000 Conviron growth chambers (Pembina, ND, USA) through the early juvenile stage (protonemal stage) and then transferred to a greenhouse at Portland State University (PSU). Mosses were grown in a "common garden" setting to reduce variation due to previous environmental conditions of the parental plant material, allowing for the partitioning of genetic versus environmental variation (Shaw, 1986). Male 
moss plants expressing mature reproductive organs (antheridia) were selected for sperm extractions. Mature antheridia typically develop a yelloworange color, enabling them to be differentiated from immature, often green antheridia (Bold 1987). Senescent antheridia are often dark or wrinkled in appearance and can also be distinguished from mature antheridia (E. E. Shortlidge, pers. obs.). Individual mature male perigonia (clusters of antheridia with surrounding modified leaves) were identified with a Leica DMZ 9.5 stereo microscope and removed from the plants with sterile forceps for sperm extraction.

\section{Sperm extraction and cellular integrity assessment}

Sperm extraction from all species began with the selection of three to 20 perigonia per plant as described in Rosenstiel and Eppley (2009). Briefly, all sperm extractions and rehydrations were performed using locally collected rainwater (average $\mathrm{pH}: 6.0 \pm 0.09 \mathrm{SEM}$ ) that was frozen and filtered and supplemented with Tetracycline (20 $\left.\mu \mathrm{g} \mathrm{ml}^{-1}\right)$ to deter bacterial growth (Rosenstiel \& Eppley, 2009). Perigonia were placed in a small pool ( $10 \mu \mathrm{l}$ per perigonium) of rainwater on multiple glass microscope slides and gently agitated with curve-tipped forceps to encourage the sperm masses to release from the antheridial jackets. Once the sperm masses were dehisced (as evident by visible whitish masses emerging from the antheridia), the slides were placed in a hydration chamber for $\sim 30 \mathrm{~m}$ to encourage continued sperm 
mass release and subsequent sperm cell release (see Paolillo, 1981 for review). The rainwater and released sperm were then pipetted from the slides into micro-centrifuge tubes, briefly centrifuged at $3,000 \mathrm{G}$ in order to pellet any fragmented plant material, and the resulting sperm cell suspensions were used for experimentation. For all experiments other than when testing for genetic variance among individuals, each sample contained representative perigonia from multiple individuals to minimize bias or effects of individual inequalities. The number of intact sperm cells was assessed using Cell Vu DRM-600 Sperm Counting Chambers (Millennium Sciences Inc., New York) at 400x, under phase contrast, using a Leica DME compound microscope. Observations of cellular integrity (intact cells) by Cell Vu chambers before and after treatments were initially confirmed by ‘live-cell' staining (SYTO ${ }$ 12; Molecular Probes, Invitrogen, Eugene, OR), coupled with an Olympus BX60 epi-fluorescence microscope. Consistent with results from phasecontrast, epi-flourescence confirmed that a fraction of moss sperm cells, postdesiccation, were found to maintain cellular integrity. Further, results of flow cytometry cell counts (Millipore, Guava, Billerica, MA), and nuclear DNA staining of sperm cells with 4,6-diamidino-2-phenylindole (DAPI; Molecular Probes, Eugene, OR), before and after sonic disruption (Cole Parmer, GEX130, 40\% power, 30s) verified cellular integrity under phase-contrast. Phase-contrast was found to be a rapid and robust way to determine sperm cell integrity, without the use of dyes, replicate counts (a minimum of two) 
were taken for each sample at each time point. In all, over 500 sperm samples were assessed over the duration of the experiments. The use of Cell Vu Sperm Counting Chambers has been found to be reliable in giving reproducible accurate sperm cell counts (Lu et al., 2007, Mahmoud et al., 1997).

As our measure of sperm tolerance to desiccation, we report sperm cell integrity rather than sperm motility, which we have used previously (Rosenstiel \& Eppley, 2009) for two reasons: 1) the ability of bryophytes to regain cellular integrity after desiccation has been well-studied (Oliver et al., 2005), and 2) in further assessing moss spermatozoa systems, we have become aware of high variance in sperm motility; we have documented bimodal distributions within individuals for all three species in this study (E. E. Shortlidge, T. N. Rosenstiel \& S. M. Eppley, unpublished). We believe that future studies should examine the relationship between sperm motility and viability in bryophytes.

\section{Dehydration and rehydration of sperm cell suspensions of $C$.}

\section{purpureus}

To assess sperm dehydration tolerance after a single dehydration event and determine whether relative humidity $(\mathrm{RH})$ during dehydration influences sperm recovery, we allowed sperm suspensions (5 $\mu$ l each, placed within 0.6 ml open Eppendorf tubes) to dehydrate in air-tight chambers. C. purpureus 
sperm was collected from 10 individuals of four populations. Suspensions were poised over one of four saturated salt solutions following protocols outlined by (Blackman et al., 1992, Winston \& Bates, 1960), or over an anhydrous desiccant, (Drierite, W.A. Hammond Drierite Company LTD) (Young 1967) for a minimum of $48 \mathrm{~h}$. The saturated solutions used included: $\mathrm{NaCl}(75 \% \mathrm{RH}), \mathrm{MgSO}_{4}(55 \% \mathrm{RH}), \mathrm{MgNO}_{3}(33 \% \mathrm{RH})$, and anhydrous $\mathrm{CaSO}_{4}$. $(<10 \% \mathrm{RH})$. By $24 \mathrm{~h}$, all samples were found to be visibly dry. To verify the stability of each $\mathrm{RH}$ treatment, temperature and $\mathrm{RH}$ within each chamber was monitored with micro data loggers (HOBO Pro v2; Onset Computer Corporation, Bourne, MA). Average RH was as expected per treatment, and average temperature was $21.15^{\circ} \mathrm{C} \pm 0.73$ (SD). For rehydration treatments, at $48 \mathrm{~h}, 5 \mu \mathrm{l}$ room-temperature rainwater was added to each tube, centrifuged for $<10 \mathrm{~s}$ at $3,000 \mathrm{G}$, and left to rehydrate for a minimum of two hours, at which time sample suspensions were gently vortexed and assessed for recovery using Cell $\mathrm{Vu}$ counting chambers as above.

\section{Addition of exogenous sugars to sperm cell suspensions of $C$.}

\section{purpureus}

In a subset of experiments, we added sucrose to sperm suspensions of $C$. purpureus to determine how the addition of exogenous sucrose influences sperm cell recovery from environmental desiccation stress. We followed the procedure outlined above with the addition of sucrose (0-25 $\mathrm{mM}$ final 
concentration) to the dehydration suspension, the rehydration suspension, or both, using C. purpureus sperm collected from six individuals from three populations. After $48 \mathrm{~h}$ (sucrose addition experiment), or $360 \mathrm{~h}$ (concentration experiment) of dehydration, the sperm samples were rehydrated and cells were assessed using $\mathrm{Cell} \mathrm{Vu}$ counting chambers as above.

\section{Comparison of sperm cell tolerance to environmental desiccation among individuals of C. purpureus}

To determine the amount of variation in tolerance among individuals to environmental desiccation, we extracted sperm suspensions (as above) from nine individuals of $C$. purpureus. All plants were grown from the juvenile stage to maturity in a common garden greenhouse environment. Three perigonia from three different ramets (clonally produced plants) were used for each of the nine individuals and maintained as separate samples, resulting in three replicate samples per individual. Sperm cells were

collected, dehydrated, hydrated, and analyzed as described above. Cells were assessed at $72 \mathrm{~h}$ using $\mathrm{Cell} \mathrm{Vu}$ counting chambers as above.

\section{Comparison of sperm cell longevity and tolerance to environmental desiccation among three moss species}

To compare longevity and tolerance to environmental desiccation among species, sperm cells were collected and maintained hydrated or dehydrated (a 
subset with $25 \mathrm{mM}$ sucrose-rainwater as the dehydration media), rehydrated, and analyzed as described above from greenhouse grown individuals of $B$.

argenteum, C. introflexus and C. purpureus, as well as field collected plants of C. introflexus. Sperm cells were assessed in rainwater (fully hydrated) over the course of $96 \mathrm{~h}$ (four days). In total, we examined sperm cell longevity in 60 samples of $B$. argenteum from three populations, 99 samples of $C$. purpureus from three populations and 131 samples of $C$. introflexus, Due to the clonal growth of mosses, we are not able to determine the number of individuals from our C. introflexus populations, but sampled broadly.

\section{Statistical analyses}

Because the variance among treatments differed, we used a one-way Welch's ANOVA to determine whether the number of intact $C$. purpureus sperm was affected by humidity treatment $(<10 \%, 33 \%, 55 \%$ and $75 \%$ relative humidity; (Morton \& Forsythe, 1974). We used a simple one-way ANOVA to determine whether the number of intact C. purpureus sperm was affected by sucrose treatment $(0.0,0.25,1.0,10.0$, and $25.0 \mathrm{mM})$. For a comparison of tolerance to dehydration among individual C. purpureus plants grown under common garden conditions, we used a mixed-model, nested ANOVA to determine whether recovery of sperm cells from dehydration was affected by population (random effect) or by individual (random effect, nested in population). For a comparison of tolerance to dehydration among species grown under common 
conditions, we used a mixed-model ANOVA to determine how species (random effect), treatment (fixed effect; hydrated, dehydrated, and dehydrated with sugar), time (fixed effect; 24, 48, $96 \mathrm{hrs)}$ ), and interactions among these factors affected sperm counts. The original cell count was included as a covariate. For significant factors in this analysis, we used posthoc analyses to determine which levels of that factor were significantly different from one another. All analyses were performed with JMP 8 (SAS Institute Inc., 2009).

\section{Results}

\section{Dehydration and ambient humidity}

A fraction of initial number of intact Ceratodon purpureus sperm cells were still intact after dehydration-rehydration events and were therefore deemed tolerant to environmental desiccation $(17 \% \pm 0.02 \mathrm{SE}$, mean for all treatments at 48 h; Fig. 2.1). Sperm was not significantly affected by humidity treatments (Fig. 2.1, $\mathrm{F}_{3,9.2}=0.22 ; \mathrm{P}=0.88$ ). The treatment with the lowest relative humidity $(<10 \%$, created using Drierite $)$ showed the least variation among replicates.

\section{Dehydration and sucrose addition}

The addition of sucrose to the suspension media significantly affected cell recovery of sperm in dehydration treatments of $C$. purpureus, but timing of 
the addition was critical (Fig. 2.2a). We found that after $48 \mathrm{~h}$ of environmental desiccation, three treatments varied significantly $(\mathrm{F}=13.17 ; \mathrm{P}$ $=0.0002)$. The samples with sucrose added to the dry-down media showed significantly higher recovery from dehydration than the samples without sucrose added at either time $(\mathrm{P}=0.0003)$, and from those with $25 \mathrm{mM}$ sucrose added to the rehydration media only $(\mathrm{P}=0.0019)$. The sperm samples with sugar added to the rehydration media only showed slightly higher recovery than those without any sucrose added, but the results were not significant. We found that the concentration of sucrose at dehydration significantly affects sperm cell recovery after an extended period of dehydration (360 h; F $=7.54 ; \mathrm{P}=0.0002$, Fig. 2.2b). Specifically, Tukey post-hoc analysis indicates that $25 \mathrm{mM}$ differed significantly from the control, $0.0 \mathrm{mM}(\mathrm{P}=0.0003), 0.25$ $\mathrm{mM}(\mathrm{P}=0.0009)$, and $1.0 \mathrm{mM}(\mathrm{P}=0.02)$, and notably was the only concentration that differed significantly from the control or from any other pairwise combination.

\section{Comparison among individuals}

Individuals of C. purpureus demonstrated variance in the fraction of their sperm that are tolerant to environmental desiccation (Fig. 2.3), although all individuals had desiccation tolerant sperm. Individuals showed significantly different recovery from environmental desiccation $(\mathrm{F}=8.53 ; \mathrm{P}=0.006)$. 
Original cell number did not significantly affect the model $(\mathrm{F}=2.72 ; \mathrm{P}=0.14)$ and populations did not vary significantly in recovery $(\mathrm{F}=0.70 ; \mathrm{P}=0.63)$.

\section{Comparison among species}

Sperm cell recovery was not significantly different among species $(\mathrm{N}=277$;

Fig. 2.4; Table 2.1). Sperm cell recovery was significantly affected by treatment and time. Cell recovery was significantly higher in sperm that remained hydrated than in those that were dehydrated with and without sucrose (Fig. 2.4). The three species of moss sperm, when hydrated, maintained a high percentage of intact cells for an extended period of time, in the range of days rather than hours (Fig. 2.4a). Cell counts decreased significantly with each increasing time point. Sperm that were dehydrated with sugar had significantly higher survival than those dehydrated without sugar (Fig. 2.4b,c). The interaction between treatment and time was also significant, as survival declined rapidly in hydrated treatment, but was not significant in either the dehydrated treatment or the dehydrated treatment with sugar (Fig. 2.4). A three-way interaction of treatment, time and species was not significant.

In a subset of treatments of $B$. argenteum and $C$. introflexus sperm, sucrose was included in the rainwater media and they were assessed for longevity at $48 \mathrm{~h}$. Interestingly, the addition of sucrose to the media significantly affected cell longevity $(\mathrm{N}=29, \mathrm{~F}=4.65, \mathrm{P}=0.01)$, at $48 \mathrm{~h}$, the 
mean recovered cell count of sperm suspended with added sucrose was lower $(51.2 \% \pm 0.004 \mathrm{SE})$ than sperm without sucrose added to the hydration media $(62.0 \% \pm 0.004 \mathrm{SE})$, suggesting sperm cells do not utilize sucrose to increase longevity.

In all cases, we found that only a fraction of sperm from any individual survived environmental desiccation, suggesting variation within individuals. This result indicates that there may be different types of sperm within a single individual. Additionally, in all three species tested there appeared be a bimodal distribution in sperm motility within individual antheridia (E.E. Shortlidge, T.N. Rosenstiel, \& S.M. Eppley, unpublished). Generally, our observations suggest that slower sperm are more tolerant to environmental desiccation, but robust correlation will require further work.

\section{Discussion}

Although the strategy of freely releasing sperm into the environment is widespread and found in a diverse array of taxa, little attention has been given to determining how motile sperm maintain viability and function in the environment. Here, we examined the ability of moss sperm to tolerate extreme dehydration, such as they might encounter in the terrestrial landscape. We found that sperm from three moss species, from three families, display a significant capacity to tolerate environmental desiccation. All three mosses produce sperm cells capable of maintaining integrity over an 
extended period of time when hydrated (Fig. 2.4a), and of recovering from environmental desiccation events (Fig. 2.4b). To our knowledge, this study represents the first to explore tolerance to environmental desiccation in free motile sperm cells. Our results suggest that moss sperm may have the potential to persist for extended periods of time on the landscape. Such findings could have significant implications for our understanding of moss mating systems and the factors that influence moss reproductive success, including the possibility of a sperm bank existing on the landscape, increased opportunity for fertilization, and a new trajectory for scientific investigation into sperm transport. Below, we discuss 1) the characteristics of tolerance to environmental desiccation in moss sperm with respect to two characteristics that are common in desiccation tolerance in plants (correlation with dry-down rate and increased tolerance with additions of sugars) and 2) variation in measured traits among moss sperm.

\section{Tolerance to environmental desiccation}

For most organisms, rate of dehydration correlates with recovery from desiccation events, with a slower rate of dehydration generally resulting in increased recovery upon rehydration (e.g., Schonbek \& Bewley, 1981, Womersley \& Ching, 1989). For instance, vegetative tissues of the desiccation tolerant moss Tortula ruralis resumed RNA synthesis quickly upon rehydration when the desiccation rate was slow versus fast, due to 
adequate time for up-regulation of cellular water-loss responses (Oliver \& Bewley, 1984b, Oliver et al., 2000a, Proctor et al., 2007). However, in reproductive plant tissues such as seeds, in which desiccation is a programmed event during maturation, desiccation tolerance increases with faster dry-down resulting in higher survival, presumably because less time is spent at intermediate water levels, where more damage can occur (Pammenter \& Berjak, 1999), for review). Our data show that the relative humidity at which moss sperm dry-down is not correlated to their recovery. This result indicates that the rate of dry-down did not affect recovery rate. We did find that the lowest relative humidity, and therefore the fastest drydown speed, yielded the least variance around the mean for cell integrity. We know that vegetative desiccation-tolerant plants that can survive rapid drydown rely on both cellular repair and cellular protective mechanisms (Oliver et al., 2000b). Whether moss sperm are able to undergo an up-regulated stress response for cellular repair, or rely only on constitutive protective mechanisms remains uncertain, but our results of similar sperm cell recovery among humidity treatments (Fig. 2.1) indicate that the mechanism may be constitutive.

While we do not see a correlation with respect to rate of dry-down and recovery in our data, our results do support the role of sugars in facilitating tolerance to extreme dehydration. We found that the fraction of cells recovering from desiccation was significantly increased by the addition of 
exogenous sucrose at dry-down (Fig. 2.2a, Fig. 2.4c), and increased as a factor of increasing exogenous sucrose concentration (Fig. 2.2b). An extensive body of literature demonstrates the role of non-reducing disaccharides in protection from desiccation damage in prokaryotes and eukaryotes (e.g., (Crowe et al., 1984, Crowe et al., 1992, Garg et al., 2002, Ingram \& Bartels, 1996, Potts, 1994). Studies have also examined the role of sugars after desiccation events in plant vegetative structures (Bewley et al., 1978, Bianchi et al., 1991, Garg et al., 2002), seeds (e.g., Koster, 1991, Koster \& Leopold, 1988, Sun et al., 1994)), and pollen (e.g., Hoekstra \& Vanroekel, 1988). While no other studies to our knowledge have examined whether sugars affect stress tolerance in plant sperm, the impact of sugars on mouse sperm has been examined. Two studies found that adding exogenous trehalose to mouse sperm increased tolerance to desiccation (Bhowmick et al., 2003, McGinnis et al., 2005). The researchers speculated that increased sperm viability was due to the relative ease in which intracellular osmolytes could come to equilibrium with a dry-down media fortified with trehalose. A similar phenomenon may have occurred in our system.

The role of sucrose in desiccation tolerance of moss sperm is only ecologically relevant if sperm encounter sucrose in the environment. Interestingly, sucrose may be present in the terrestrial environment in which moss sperm are released. Not only does sucrose accumulate inside moss cells that endure desiccation and freezing events (Proctor et al., 2007, Rütten \& 
Santarius, 1992), but sucrose is a major component of the pulse-released suite of compounds found after a rewetting event, post desiccation (Coxson et al., 1992). Furthermore, sucrose is a constituent of the exudates from moss archegonia (Pfeffer, 1884, Kaiser et al., 1985, Ziegler et al., 1988) and potentially of other vegetation and fruits found in the plant canopy above. Hence, there is evidence of a multi-faceted role for sucrose in the ecophysiology of mosses.

\section{Sperm variation}

While we speculate that an individual moss will be at an advantage if its sperm tolerates environmental desiccation, genetic variation must be present for selection to act on the trait. Genetic variation for desiccation tolerance among populations and individuals has been found in many organisms, including plants (e.g., Basnayake et al., 1993, Glazer et al., 1991, Hoffmann \& Harshman, 1999, Jurenka et al., 2007). In Drosophila melanogaster, (Hoffmann \& Parsons, 1989) found that desiccation resistance is up to $60 \%$ heritable. High levels of genetic variation suggest this trait would respond rapidly to selection, which experiments confirmed (e.g., Chippindale et al., 1998). We found that variation in sperm tolerance to environmental desiccation does not occur among examined species, but does occur among individuals within a species (Table 2.1; Fig.2.3). Our mosses were grown in a common-garden, in uniform greenhouse conditions although collected from 
various locales, therefore differences among individuals are likely genetic, although we cannot rule-out increased variance in maternal effects, as we collected individuals from within and among populations. However, the most likely explanation is that genetic variation exists among individuals for sperm tolerance to environmental desiccation, leading to the question of why such a potentially adaptive trait would not sweep through a population.

Researchers have suggested that an organism's ability to tolerate any stress, not just desiccation, is inversely proportional to its ability to compete because of trade-off between stress tolerance and growth or metabolic rate (Arendt, 1997, Chapin et al., 1993, Grime, 1977, Hoffmann \& Parsons, 1989).

Research in echinoderms implies trade-offs in sperm velocity versus life-span (Levitan, 2000), suggesting that energetically-based life-history trade-offs can occur in sperm. Further research will be essential to determine whether there are trade-offs between differential stress tolerance in moss sperm and other sperm traits and how environmental conditions may inform these relationships.

We also have found that only a fraction of sperm from any individual of our three species survived desiccation, suggesting variation within individuals among sperm in tolerance to environmental desiccation, and perhaps suggesting the possibility of variable sperm types within a single moss individual. Heteromorphic sperm are widespread among animal taxa, including in such unrelated lineages as mollusks, insects, echinoderms, and 
vertebrates (e.g., Au et al., 1998, Buckland-Nicks et al., 1999, Hayakawa et al., 2007, Hodgson, 1997, Lee \& Wilkes, 1965, Lutzen et al., 2004, Van Look et al., 2007), many of which reproduce via external fertilization. Heteromorphic sperm also occur in angiosperms (e.g., Gou et al., 2009, Hirano \& Hoshino, 2010, Saito et al., 2002, Weterings \& Russell, 2004). Heteromorphic pollen in angiosperms, and sperm in animals have been suggested to be convergent, having both evolved via sexual selection to increase male success (Till-Bottraud et al., 2005). Sperm with variable DNA content have been documented within individual bryophytes (Bernhard \& Renzaglia, 1995) and researchers have speculated that due to the prevalence of heterospermy across animal taxa, variable sperm should also be present within non-seed plants (Till-Bottraud et al., 2005). Although the moss sperm are formed through a mitotic pathway, there is potential for variation in spermatogenesis such as during cytoplasmic reduction, or as a result from environmental treatments, as found in some ferns (Southworth \& Cresti, 1997 for review). The ecological significance of heteromorphic sperm is unknown in angiosperms (Weterings \& Russell, 2004). However, in animals, which like moss also release naked sperm, variation in sperm type within an individual is widespread. Among animals, heteromorphic sperm can vary in DNA content and fertilization ability, and have been hypothesized to have important co-functions in reproduction, e.g. protect co-sperm from harsh environmental conditions, aid in sperm competition, and facilitate movement 
of co-sperm in aqueous environments (Buckland-Nicks, 1998, Hayakawa, 2007, Higginson \& Pitnick, 2011, Silberglied et al., 1984). Whether the moss sperm in our study are truly heteromorphic with different developmental pathways, constitutive defenses, DNA contents, and/or abilities to fertilize, or whether they vary in tolerance to desiccation across an environmental spectrum remains to be seen. Future studies should consider not only the possibility of heteromorphic moss sperm and its implications for the evidently complex reproductive ecology of mosses, but also seek to understand the fitness consequences for individuals possessing functional variation in sperm traits.

\section{Acknowledgements}

We thank H. Le, C. Rupert, and J. Storey for laboratory support. Research was supported by the U.S. National Science Foundation (DEB 0743461 to S. Eppley and IOS 0719570 to T. Rosenstiel) and the 3M Corporation.

This chapter is reprinted from published work:

Shortlidge EE, Rosenstiel TN, Eppley SM (2012) Tolerance to environmental desiccation in moss sperm. New Phytologist, 194, 741-750.

Shortlidge has full rights to reprint the content in agreement with John Wiley and Sons and provided by Copyright Clearance Center, 2014. License no. 3440270401212 . 


\section{Tables and Figures}

Table 2.1 Tolerance to desiccation in moss sperm. To determine how species (random effect; Bryum argenteum, Campylopus introflexus, and Ceratodon purpureus), treatment (fixed effect; hydrated, dehydrated or dehydrated with sucrose), time (fixed effect; $24,48,96 \mathrm{~h}$ ), and interactions among these factors affected sperm cell recovery, we used a mixed-model ANOVA $(\mathrm{N}=277)$. The original cell count before treatment was included as a covariate. Significant P-values are indicated in bold.

\begin{tabular}{lcccc} 
& df & SS & F & P \\
\hline \hline Species & 1 & 0.35 & 3.74 & 0.15 \\
Time & 1 & 0.04 & 0.85 & 0.38 \\
Treatment & 1 & 6.72 & 181.48 & $<\mathbf{0 . 0 0 0 1}$ \\
Species*Time & 3 & 0.27 & 2.25 & 0.17 \\
Species*Treatment & 3 & 0.16 & 1.29 & 0.37 \\
Time*Treatment & 3 & 1.23 & 8.19 & $\mathbf{0 . 0 0 3}$ \\
Species*Time*Treatment & 7 & 0.28 & 1.34 & 0.23 \\
Original Cell Count & 1 & 0.001 & 0.05 & 0.83 \\
\hline \hline
\end{tabular}


Figure 2.1 Effects of dry-down rate on sperm cell recovery from environmental desiccation. Fraction of C. purpureus sperm intact after $48 \mathrm{~h}$ in four dehydration-rehydration treatments with variable relative humidity $(\mathrm{N}=30)$. Error bars represent data mean $\pm \mathrm{SE}$. Means do not vary significantly among treatments.

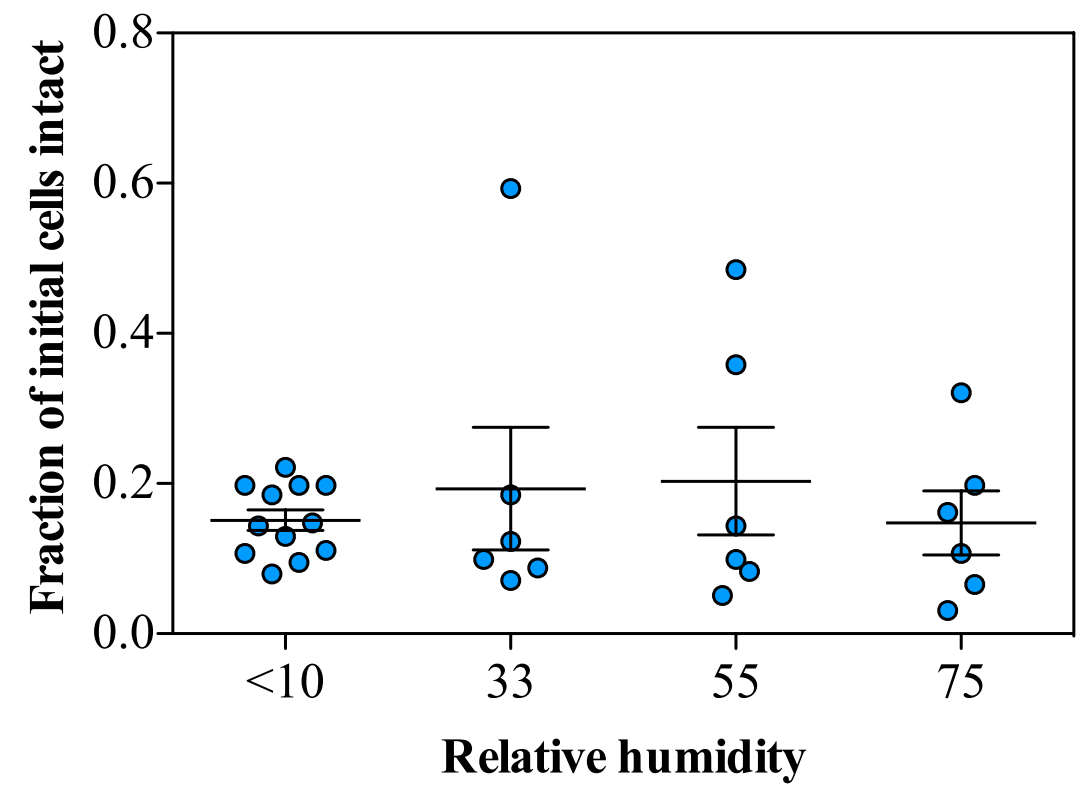


Figure 2.2 Exogenous sucrose aids sperm cells in recovery from environmental desiccation events. Fraction of C. purpureus sperm cells intact after dehydration-rehydration treatment with or without the addition of sucrose. a) Fraction of sperm intact after $48 \mathrm{~h}$ in one of three treatments: 1) -/- (rainwater for dehydration and rehydration), 2) -/+ (rainwater for dehydration, sucrose was added to rehydration media), 3) +/- (sucrose was added to dehydration media, rainwater for rehydration) $(\mathrm{N}=24, \mathrm{P}<0.0003)$. Bars represent data mean, whiskers are minimum to maximum values. b) Fraction of sperm intact after $360 \mathrm{~h}$ of dehydration-rehydration treatment with increasing concentrations of sucrose added to the dehydration media ( $\mathrm{N}$ $=36 ; \mathrm{P}=0.0002)$. Error bars represent data mean $\pm \mathrm{SE}$.

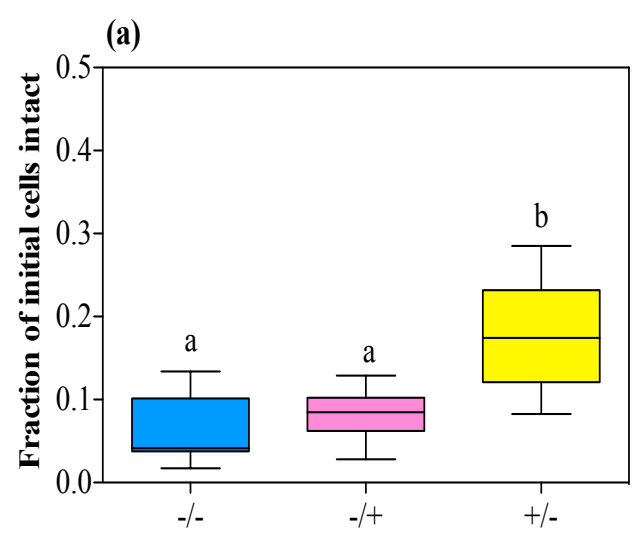

Dehydration/Rehydration treatment

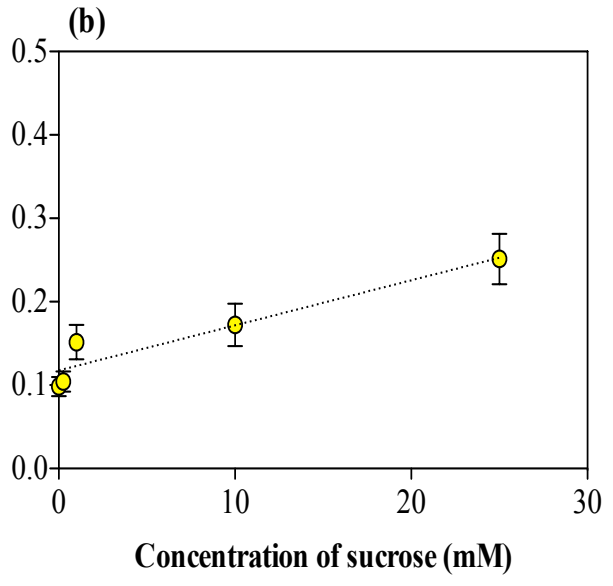


Figure 2.3 Variation among individuals in sperm cell recovery from environmental desiccation. Fraction of C.purpureus sperm intact after $72 \mathrm{~h}$ dehydration-rehydration treatment from nine individuals of four populations. $(\mathrm{N}=18, \mathrm{P}=0.006)$. Error bars represent data mean $\pm \mathrm{SE}$.

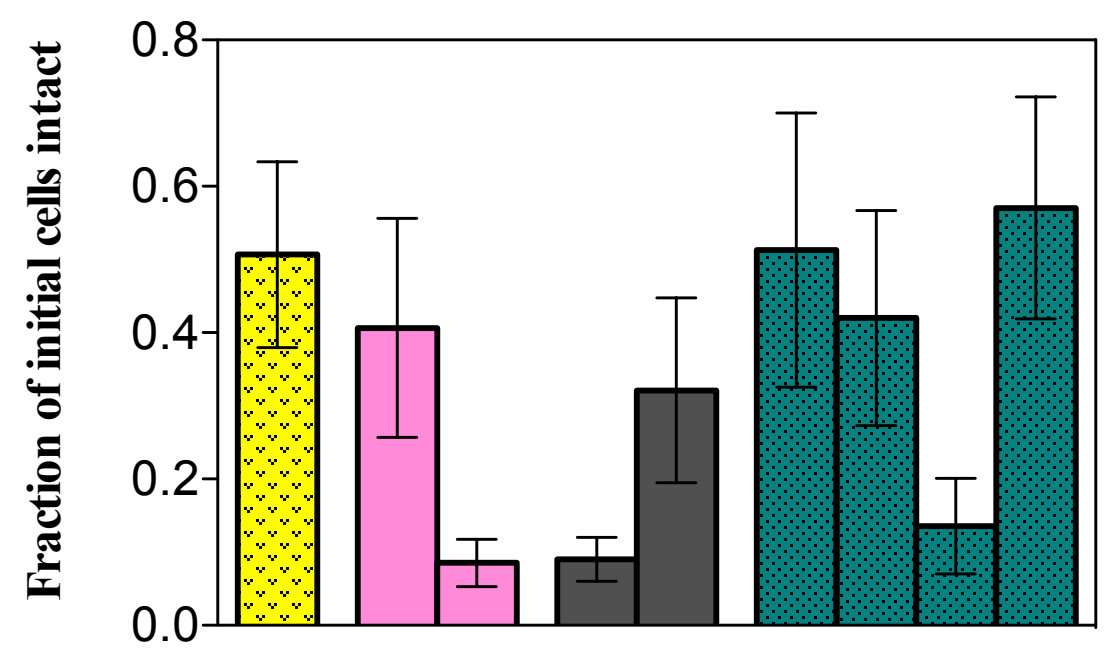

Nine individuals of four populations 
Figure 2.4 Effects of time and dehydration treatments on sperm cell integrity in three species of mosses. Closed circles indicate B. argenteum, open circles indicate C. purpureus, and squares indicate C. introflexus (a) Hydration treatment: fraction of sperm still intact over time. The sperm were stored in rainwater and assayed at 24, 48 and $96 \mathrm{~h}$ after sperm extraction $(\mathrm{N}=109)$. Error bars represent data mean $\pm \mathrm{SE}$. (b) Dehydration treatment: fraction of sperm still intact after dehydration-rehydration events. The sperm was rehydrated at 24, 48, and $96 \mathrm{~h}$ and assessed for cellular integrity $(\mathrm{N}=125)$. Error bars represent data mean $\pm \mathrm{SE}$. (c) Sucrose and dehydration treatment: Fraction of sperm cells intact after dehydrationrehydration events with sucrose added to dehydration media The sperm was rehydrated at 24, 48, and $96 \mathrm{~h}$ and assessed for cellular integrity $(\mathrm{N}=56)$. Error bars represent \pm SE.

(a)

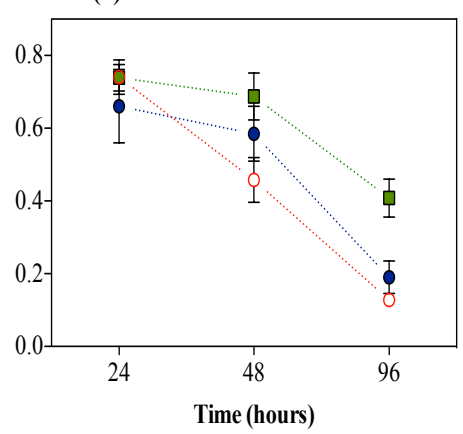

(b)

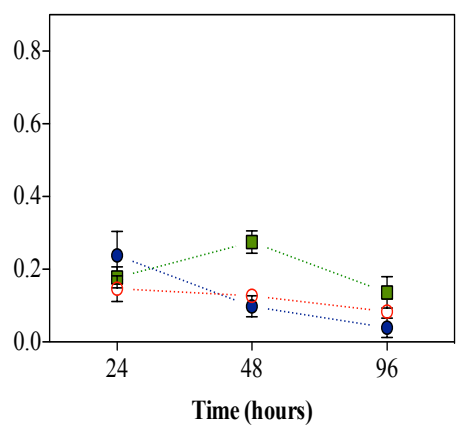

(c)

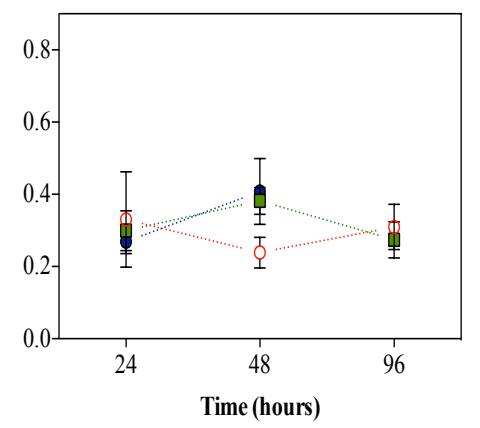




\title{
Chapter 3
}

\section{Sex-specific volatile compounds influence microarthropod-mediated fertilization of moss}

\begin{abstract}
Sexual reproduction in non-vascular plants requires that unicellular freemotile sperm travel from male to female reproductive structures across the terrestrial landscape (Paolillo, 1981). Recent data suggest that microarthropods can disperse sperm in mosses (Cronberg et al., 2006); however, little is known about the chemical communication, if any, involved in this interaction or the relative importance of microarthropod dispersal compared to abiotic dispersal agents in mosses. Here, we show that tissues of the cosmopolitan moss Ceratodon purpureus emit complex volatile scents, similar in chemical diversity to those described in pollination mutualisms between flowering plants and insects; that the chemical composition of $C$. purpureus volatiles are sex-specific; and that moss-dwelling microarthropods are differentially attracted to these sex-specific moss volatile cues. Further, using experimental microcosms, we show that microarthropods significantly increase moss fertilization rates, even in the presence of water spray, highlighting the important role of microarthropod dispersal in contributing to moss mating success. Taken together, our results suggest the presence of a
\end{abstract}


previously unknown scent-based "pollination-like" syndrome between two of Earth's most ancient terrestrial lineages, mosses and microarthropods. 


\section{Introduction}

The origin of bryophytes (mosses, liverworts, and hornworts) during the upper Ordovician represents a significant event in the evolution of life (Kenrick \& Crane, 1997, Nickrent et al., 2000), leading to the diversification of terrestrial organisms. From a mating systems perspective, bryophyte evolution resulted in sexual reproduction partially escaping the aquatic environment. As an ever present reminder of the aquatic origins of sex, sexual reproduction in mosses requires that free-motile sperm 'swim' with the aid of water across the terrestrial landscape to fertile females (Muggoch \& Walton, 1942, Paolillo, 1981). This model of 'swimming sperm' has led to the general view that sperm dispersal among bryophytes is quite limited, with most fertilization occurring within about $10 \mathrm{~cm}$ (Longton, 1976, Shaw \& Goffinet, 2000). However, recent research using the moss Bryum argenteum demonstrates that moss sperm can be dispersed by microarthropods (Cronberg et al., 2006), specifically springtails and oribatid mites which are common inhabitants of moss patches worldwide (Andrew et al., 2003). This new research builds on earlier, often overlooked work suggesting that arthropods may act as ecologically relevant sperm transport vectors (Muggoch \& Walton, 1942). Further, recent data show that moss sperm can be more long-lived and stress tolerant than previously believed (Rosenstiel \& Eppley, 2009, Shortlidge et al., 2012), potentially allowing sperm to survive during long-distance microarthropod dispersal. Although microarthropods 
are known to use volatile cues in foraging and for communication (Raspotnig et al., 2005, Verhoef et al., 1977), little is known about whether microarthropods may also use chemical cues to facilitate sexual reproduction in mosses.

\section{Materials and Methods Summary}

To examine volatile profiles in these mosses, gas chromatography was carried out using a LECO Pegasus 4D GC×GC-TOFMS system (LECO, St. Joseph, MI, USA). For each sample, 30-40 mg of intact (non-wounded) moss shoots was allowed to equilibrate in a glass vial for 120 minutes. Headspace sampling was then carried out for 60 minutes with a solid phase microextraction (SPME) fiber; thermal desorption of the SPME fiber and analysis by GC×GC-TOFMS followed, as described in (Pankow et al., 2011). Data are based on Ceratodon purpureus plants collected in Oregon and maintained in greenhouse culture.

To determine springtail preference for male versus female $C$.

purpureus samples of intact shoots, we conducted two sets of preference assays. First, for preference assays of whole moss patches, protocols were modified from well-established springtail food preference assays in Petri dishes (Sadaka-Laulan et al., 1998, Thimm \& Larink, 1995), and we used C. purpureus plants collected in Oregon and maintained in greenhouse culture. Second, for volatile preference assays, we used a custom-constructed static- 
air olfactometer designed for springtails (Staaden et al., 2011), and $C$.

purpureus plants were collected directly from the field in Oregon. We used two springtail species, Folsomia candida, and Sinella curviseta, for both sets of assays.

To determine the effect of springtails and water spray on moss fertilization, we maintained microcosms of C. purpureus ( $\mathrm{N}=72$ microcosms) and $B$. argenteum ( $\mathrm{N}=36$ microcosms) for approximately 15 weeks in a factorial design with treatments of added springtails and water spray, counting the number of sporophytes after initial sporophyte formation. $C$. purpureus and B. argenteum plants were collected in Oregon, Arizona, and Kentucky and maintained in greenhouse culture.

\section{Study system}

Ceratodon purpureus (Hedw.) Brid. and Bryum argenteum Hedw. are nearly cosmopolitan species, with dioicous breeding systems (Lawton, 1971). For this study, in 2009, we collected plants from three C. purpureus populations in the Portland, Oregon metro area from: 1) northeast Portland, 2) the Portland State University campus, and 3) a farm in North Plains, Oregon, with populations a minimum of $5.8 \mathrm{~km}$ apart. The $B$. argenteum plants were collected from 2008-2009 from four populations from: 1) southern Arizona, 2) the University of Kentucky campus, 3) southwest Portland, Oregon, and 4) downtown Portland, Oregon, with populations a minimum of $1.9 \mathrm{~km}$ apart. Plants were collected from field populations and grown in the Portland State 
University greenhouse in 6.4-x-6.4 cm pots for at least three months for all experiments except the olfactometer experiment (for which plants were collected directly from the field). Plants from the C. purpureus northeast Portland and Portland State University populations and the B. argenteum Arizona population were grown from spores (single spore isolations) to ensure separate individuals from these smaller populations, while the other plants were grown from single shoot cuttings collected from as far apart as possible within populations. Two commercially-available species of springtails, Folsomia candida and Sinella curviseta, were reared in airtight containers with natural charcoal, de-ionized water, and yeast, and they were kept in the same growth chambers in which the microcosm experiments were performed (see subsection d). F. candida is a model springtail species, growing in soils worldwide (Fountain \& Hopkin, 2005), and it occurs in high densities in soil and moss communities in the Pacific Northwest region of the USA and Canada (Johnson \& Wellington, 1980). S. curviseta is an emerging model system as it occurs in sites where F. candida is rare (Xu et al., 2009). Both species were used in all springtail experiments.

\section{Scent collection and GC×GC-TOFMS analyses}

To examine the volatile scent profiles of intact moss tissue, we used static headspace, a method which is sensitive to identifying small quantities of compounds but cannot be used for quantification of amounts of compounds in 
volatile signatures. For each tissue sample, 30-40 mg of intact (non-wounded) shoots was carefully removed from pots. Each sample was placed into a 2.0 $\mathrm{ml}$ screw-top glass vial and allowed to equilibrate for 120 minutes. A solid phase micro-extraction (SPME) fiber (polydimethylsiloxane/divinylbenzene, $65 \mu \mathrm{m}$ coating, Sigma-Aldrich, St. Louis, MO) was then exposed to the headspace for an additional 60 minutes; results did not change appreciably with additional exposure time (results not shown). Each analysis began by inserting the SPME fiber into the injector (with "SPME liner") of a twodimensional gas chromatograph (LECO Pegasus 4D GC×GC-TOFMS system; St. Joseph, MI, USA); the column and analytical conditions used were as previously described for biogenic volatiles (Pankow et al., 2011). Trace contaminants from ambient air blanks were identified and removed from each of the comprehensive volatile profiles prior to further data analysis. Comprehensive GCxGC-TOFMS analysis was chosen in order to minimize any a priori assumptions about the chemical nature of the volatile compounds emitted by the moss system.

To determine whether male and female plants of $C$. purpureus differed in scent composition, we compared overall variation in chemical composition between the sexes, using males and females from each of the three Portland, Oregon populations ( $\mathrm{N}=22$ plants total). For all analyses, we used plants that were producing gametoecia (perichaetia and perigonium, female and male sex organs, respectively, with clusters of modified leaves), and we 
enriched for these structures in the samples. However, initial screens of plant material without gametoecia suggest that male and female plants show a similar difference in volatile composition (data not shown); further work is required to determine sex-specific ontological and morphological variation in volatile emission rate, site of production, and phenological variation. The full list of volatile compounds we observed in the head-space analyses of $C$. purpureus is given in Table A.A1.

\section{Springtail preference assays}

To determine whether springtails prefer one reproductive moss sex or the other and whether this was due to volatile cues, as suggested by the GC×GCTOFMS analyses, we conducted two sets of preference assays with the moss C. purpureus. First, we used protocols modified from well-established springtail food preference assays to construct preference chambers from Petri dishes (Sadaka-Laulan et al., 1998, Thimm \& Larink, 1995), and we conducted preference assays in these dishes comparing male and female intact (non-wounded) reproductive C. purpureus samples. For each assay, we used a 55-x-15 mm Petri dish; placed a $55 \mathrm{~mm}$ diameter piece of filter paper in the bottom of the dish; and placed two smaller pieces of filter paper, separated by $1.5 \mathrm{~cm}$, on top of the larger filter paper. The two comparison samples (5 mm diameter moss patches of intact shoots) were placed on the two smaller filter papers. Moss samples were from two Oregon populations 
(Northeast Portland and Portland State University populations), and both males and females were producing gametoecia. Using a metal spatula, we placed 20-40 springtails in each dish between the moss samples, wrapped the dishes in parafilm, darkened them with foil, and placed them in the growth chamber in which the springtails were reared. After 120 minutes, we removed the moss samples and filter paper and determined the number of springtails within each moss sample, the number of springtails that did not occupy a moss sample, and the number of moss shoots and moss reproductive structures per sample. Plants were dried in a drying oven at $60{ }^{\circ} \mathrm{C}$ for 48 hours and dry weight was determined. We conducted 24 assays with 491 springtails choosing specific moss samples. Springtails were never reused in assays. We found no significant difference in dry weight between male and female moss samples $(\mathrm{P}=0.95 ;$ mean $\pm \mathrm{SE}=8.8 \pm 1.2 \mathrm{mg}$ and $9.1 \pm 1.3 \mathrm{mg}$, respectively); however male moss samples had significantly more shoots and gametoecia per shoot than did females $(\mathrm{P}=0.03$; mean $\pm \mathrm{SE}=25.7 \pm 1.88$ and $20.3 \pm 1.6$, respectively for shoots; $\mathrm{P}=0.001 ; 1.46 \pm 0.32$ and $0.22 \pm 0.02$, respectively for gametoecia per shoot).

To determine whether the preference we measured for female plants was due to springtails perceiving a volatile cue or another type of assessment (e.g., visual), we set up a second set of assays using a static air olfactometer with intact (non-wounded) male and female C. purpureus samples. The olfactometer was modified after (Steidle \& Schöller, 1997), with additional 
modification for springtails following (Staaden et al., 2011) The olfactometer was made of clear acrylic pipe with two sample compartments divided by a vertical plate. A walking arena for the springtail was placed above the compartments, with the springtails separated from the samples by a wetted opaque filter, to obscure visual choice. For each assay, a male and a female moss sample (15mm diameter moss patches of intact shoots) were added to separate compartments of the olfactometer. Plants were field collected in May 2012 from multiple sites within a large population $\left(>6,000 \mathrm{~m}^{2}\right)$ in North Plains, Oregon, and plants were nearing the end of the fertilization season, with females producing a few gametoecia and many new sporophytes, and males producing many gametoecia with ripe antheridia. Using a spatula, we placed 20-40 springtails on the walking arena; the olfactometer was closed and placed in the dark; and we recorded the springtails' choices every 30 minutes for 120 minutes. We conducted 10 assays with 276 springtails choosing specific moss samples. We dried and weighed the moss samples, as for the previous assays. We found no significant difference in dry weight or number of shoots between male and female samples $(\mathrm{P}=0.99$; mean \pm $\mathrm{SE}=176.31 \pm 18.86 \mathrm{mg}$ and $160.00 \pm 29.84 \mathrm{mg}$, respectively for dry weight; $\mathrm{P}=0.53 ; 0.21 \pm 0.03$ and $0.21 \pm 0.04$, respectively for shoots). Male moss samples did differ significantly from female samples in the number of gametoecia per shoot $(\mathrm{P}=0.0003 ;$ mean $\pm \mathrm{SE}=0.43 \pm 0.07$ and $0.21 \pm 0.04$, respectively). 


\section{Bryophyte microcosms}

To determine the effect of springtail and water spray on sperm dispersal in mosses, we set up factorial experiments in which we manipulated springtail and water spray levels in C. purpureus and B. argenteum, and we counted sporophyte number as an estimate of fertilization success following (Mishler, 1990). To establish microcosms, we propagated the moss on a substrate of a 2:1 mixture of propagation grade sand and peat moss. The mosses were propagated by chopping fresh plant material, and distributing the chopped material evenly among microcosms (6.4-x-6.4 cm pots), with microcosms containing either C. purpureus or B. argenteum. For each moss species, microcosms contained plant material from a mix of 3-5 populations and were composed of both males and females. The microcosms were placed in seedling trays and covered in humidomes to allow for under-watering of microcosms and to create an enclosed habitat that was conducive to growth for both the springtails and mosses. The experiments were set up in Adaptis 1000 Conviron growth chambers (Pembina, ND, USA), enabling us to control for temperature, light, and relative humidity (14 hours light/10 hours dark cycles with $18^{\circ} \mathrm{C}$ light $/ 8^{\circ} \mathrm{C}$ dark; 150 micromoles photons $\mathrm{m}^{-2} \mathrm{~s}^{-1}$; and $65 \%$ constant humidity). The microcosms were subjected to one of four treatments: 1) springtails only, 2), water spray only 3) springtails and water spray, 4) neither springtails nor water spray. Microcosms of water spray and no-spray treatments were evenly distributed among trays of one of two designations 
(springtails or no-springtails). One liter of water was maintained in the base of each tray, and each tray was covered with a humidome lid. Trays were rotated every two weeks within growth chambers to control for chamber effects. Water spray was applied once/week with a squirt bottle containing room temperature spring-water. After 80 days in microcosms, an excess of algae accumulated in the $B$. argenteum microcosms, and the spray treatment was intermittent to allow the plants to recover; however, the spray was maintained at least every 14 days. Springtails ( 20/microcosm) were added from stock cultures to all appropriate treatment trays once every 2-3 weeks and were observed living in the treatment microcosms in the weeks after application. We counted the number of sporophytes in each treatment after initial sporophyte formation (which we defined as the day when at least $15 \%$ of microcosms had sporophytes). The B. argenteum was started in August 2010 and took 44 days to reach initial sporophyte formation after planting. The C. purpureus were run as two separate experiments (starting in July 2010 and September 2010) with multiple trays per treatment for each set. One set took 231 days while the second set took 179 days to reach initial sporophyte formation after planting.

We used a third experiment of C. purpureus ( $\mathrm{N}=32$ microcosms) with the same treatments to test for variation among springtail treatments in 1) number of gametoecia; 2) chlorophyll fluorescence PSII efficiency (Fv/Fm); and 3) plant nitrogen content. We found no significant differences among 
springtail treatments in any of these measures, in our statistical model with springtails nested in tray and including tray as a factor, although adding springtails increased sporophyte production, as in the other experiments. These data suggest that the springtail addition did not enhance reproductive expression leading to more sporophyte production, nor did it alter overall plant health before sporophyte formation, consistent with a role of springtails in mediating sperm transfer. Data and statistics are presented in Appendix A, Fig. A.A4, Table A.A2.

\section{Data analysis}

Multivariate analysis was used to discriminate among volatile scent profiles (Van Dam \& Poppy, 2008). Specifically, nonmetric multidimensional scaling (NMDS) and analysis of similarities (ANOSIM) were performed using the Rstatistical package to test for differences among volatile scent composition between male and female plants. Prior to analyses, individual volatile compounds were sorted into one of 21 IAUPAC compounds classes and square-root transformed. All other analyses were conducted using JMP Version 10.0 (SAS Institute, 2012). T-tests were used to test for differences in the average number of VOCs released between male and female plants. We used G-tests to determine whether springtails preferentially chose between the two samples for each of the two types of preference assays (female vs male samples in Petri dishes or in the olfactometer) at 120 minutes. We used 
springtail choice data from the olfactometer assays at 120 minutes only because there was no significant difference among time points. For preference assays, we used t-tests to determine whether male and female moss patch samples differed in dry weight, shoot number, and number of gametoecia per shoot. We used logit analysis to determine the effect of the springtail treatment, the water spray treatment, and the interaction between these treatments on the fraction of microcosms with sporophytes. We included tray, nested in springtail treatment, in the model. We included species and interactions with species but found that these were not significant and were dropped from the model. We also used a similar mixed-model nested ANOVA to analyze how the number of sporophytes per microcosm (log transformed) were affected by these factors, and we found that no factors lost or gained significance by changing our outcome variable, suggesting that these results are robust (results not shown).

\section{Results}

Here, we assess the potential role of moss volatile cues and microarthropods (springtails) in mediating sperm dispersal in Ceratodon purpureus, a model cosmopolitan moss species with separate sexes. First, to fully capture the suite of possible volatile organic compounds (VOCs) emitted from intact (nonwounded), sexually-expressing male and female plants, we characterized headspace VOCs using two-dimensional gas chromatography - time of flight 
mass spectrometry (GC×GC-TOFMS). We found that for all sampled populations, female plants released a significantly greater number of VOCs than male plants $(104.00 \pm 9.27$ and $29.86 \pm 8.21$, respectively; $\mathrm{P}<0.0001$, Fig. 3.1). In addition, analyses of VOC composition revealed significant sexspecific differences (ANOSIM: $\mathrm{R}=0.79 ; \mathrm{P}=0.001$, stress value=3.8; Fig. 3.2). A surprising diversity of volatile compounds was identified in head-space analysis using our GC×GC-TOFMS approach, and many of these compounds have been previously identified in floral scents of flowering plants (Knudsen et al., 1993). Further details on sex-specific VOCs are provided in Appendix A, Fig. A.A1.

Second, to determine whether springtails were differentially attracted to the observed sex-specific VOC composition, we conducted a series of preference assays using intact (non-wounded) samples of male and female $C$. purpureus. In the first set of preference assays, springtails were given choices between male and female moss samples in Petri dishes, and springtails were significantly more likely to choose intact reproductive female plants over intact reproductive male plants $(\mathrm{G}=37.6 ; \mathrm{P}<0.0001$; Fig. 3.3). This result is similar to the result found by Cronberg et al. in which springtails and mites marginally preferred female to male reproductive $B$. argenteum plants. To confirm that springtail preference for female plants was due to femalespecific volatile cues, we used an olfactometer for additional preference assays, allowing microarthropods to assess scents produced by the samples 
but not allowing visual or physical cues. In the olfactometer assays, springtails chose intact reproductive female plants significantly more frequently than intact reproductive male plants $(\mathrm{G}=58.1 ; \mathrm{P}<0.0001)$. These results reveal the surprising role of volatile cues in influencing microarthropod choice of intact female moss plants.

Finally, we used a series of microcosm experiments where we manipulated springtail abundance and water spray to assess the importance of biotic versus abiotic factors in promoting sperm dispersal and fertilization in mosses. For this experiment, we used C. purpureus, as well as $B$. argenteum, the moss species for which springtail-mediated sperm dispersal has been previously demonstrated (Cronberg et al., 2006) Our results show that for both moss species, the addition of either springtails or water spray significantly increased the number of sporophytes formed per microcosm and the fraction of microcosms that developed sporophytes (Fig. 3.4). Further, the combination of treatments had a pronounced synergistic effect, more than doubling the effect of either treatment alone (Fig. 3.4c). These results highlight the significant role of microarthropods in facilitating fertilization in mosses, presumably through enhanced sperm transport.

\section{Discussion}

Plant-insect interactions were key to the diversification of flowering plants (Crepet, 1984), with floral scent representing a primary mode of 
communication between plants and their pollinators (Raguso, 2008, Schiestl, 2010). Our data suggest that mosses, despite their lack of flowering structures, may similarly utilize volatile scents as cues to manipulate microarthropod behavior, resulting in increased moss fertilization. Therefore, we suggest the potential of a significant "pollination-like" syndrome between microarthropods and mosses involving volatile scent cues.

Sex-specific floral scents have been found in over 20 species of flowering plants with separate sexes, and several hypotheses have been proposed to explain this pattern (Ashman, 2009). One hypothesis suggests that the most mate-limited sex is likely to evolve the greatest floral scent (Ashman, 2009). If this theory extends to bryophytes, then our results suggest that female mosses are more mate-limited than males, a likely scenario given the highly female-biased population sex ratios of these species (Shaw \& Gaughan, 1993, Stark et al., 2010), as is typical in mosses (Bisang $\&$ Hedenas, 2005). Another hypothesis suggests that differential pollinator rewards between the sexes may lead to selection for differential cues, including sex-specific VOCs (Hemborg \& Bond, 2005). If during the normal course of their movements, microarthropods inadvertently pick up released moss sperm from water film (Muggoch \& Walton, 1942), or if moss sperm are food reward for microarthropods, similar to pollen in some plant-pollinator systems, then the reward and cues for male and female moss plants are likely to be different. Females, for instance, may produce high concentrations of 
sucrose or fatty acids as a reward, as has been suggested (Cronberg et al., 2006). We have not yet distinguished between the composition and amounts of VOCs produced by the reproductive structures and the entire plant, nor between the moss tissue and any associated phyllospheric microbes. Sexspecific mutualistic interactions do occur between hosts and microbes (Varga \& Kytoviita, 2008) and can induce sex-specific VOC differences in the host (Voigt et al., 2005). Such interactions may also exist in bryophytes. Further studies are needed to establish the fundamentals of this moss-microarthropod signaling system, including determining which specific VOCs, or suites of VOCs, are most important for signaling as well as pinpointing the cells responsible for the production of key volatile cues. As mosses and microarthropods represent two of Earth's most ancient co-occurring terrestrial lineages, it is important to consider the potential role a "pollination-like" syndrome may have played in shaping the evolutionary ecology of moss mating systems.

\section{Acknowledgments}

We are grateful to A. Melnychenko, J. Pankow, W. Luo and L. Isabelle for excellent analytical assistance, to C. Rupert for assistance with the preference assays, to L. Stark and N. McLetchie for plant material, and to anonymous reviewers for comments on earlier drafts of this manuscript. 
Funding was provided by the 3M Corporation and National Science Foundation (DEB-0743461 to S. Eppley and IOS-0719570 to T. Rosenstiel).

This chapter is reprinted from published work:

Rosenstiel TN, Shortlidge EE, Melnychenko AN, Pankow JF, Eppley SM (2012) Sex-specific volatile compounds influence microarthropodmediated fertilization of moss. Nature, 489, 431-433.

As an author of this original (primary) research paper, Nature Publishing Group does not require permissions to reprint the article. 


\section{Figures}

Figure 3.1 Sex-specific volatile profiles. Representative two-dimensional GC×GC-TOFMS chromatograms of volatile compounds from intact shoots of $\mathbf{a}$. one reproductive male and $\mathbf{b}$. one reproductive female of the cosmopolitan moss Ceratodon purpureus.
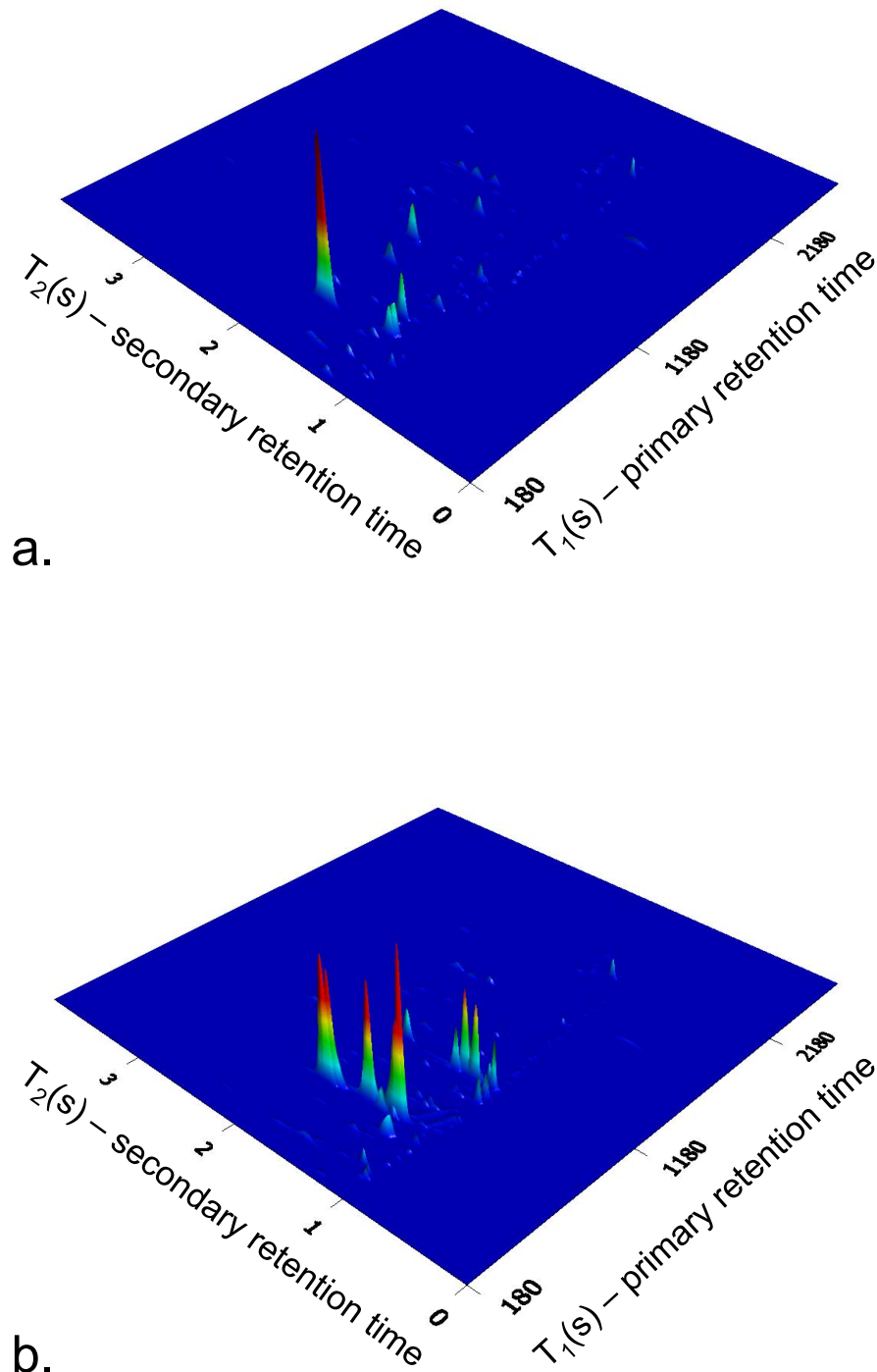
Figure 3.2 Significant differences in volatile composition. Nonmetric multidimensional scaling (NMDS) of volatile scent profiles of reproductive male and reproductive female plants of Ceratodon purpureus. Symbols represent scent profiles of individual males $(\Delta)$ and females (o), respectively. $\mathrm{N}=22 \mathrm{GC} \times \mathrm{GC}-\mathrm{TOFMS}$ analyses, stress $=3.8$. ANOSIM between male and female scent profiles: $\mathrm{R}=0.79 ; \mathrm{P}=0.001$.

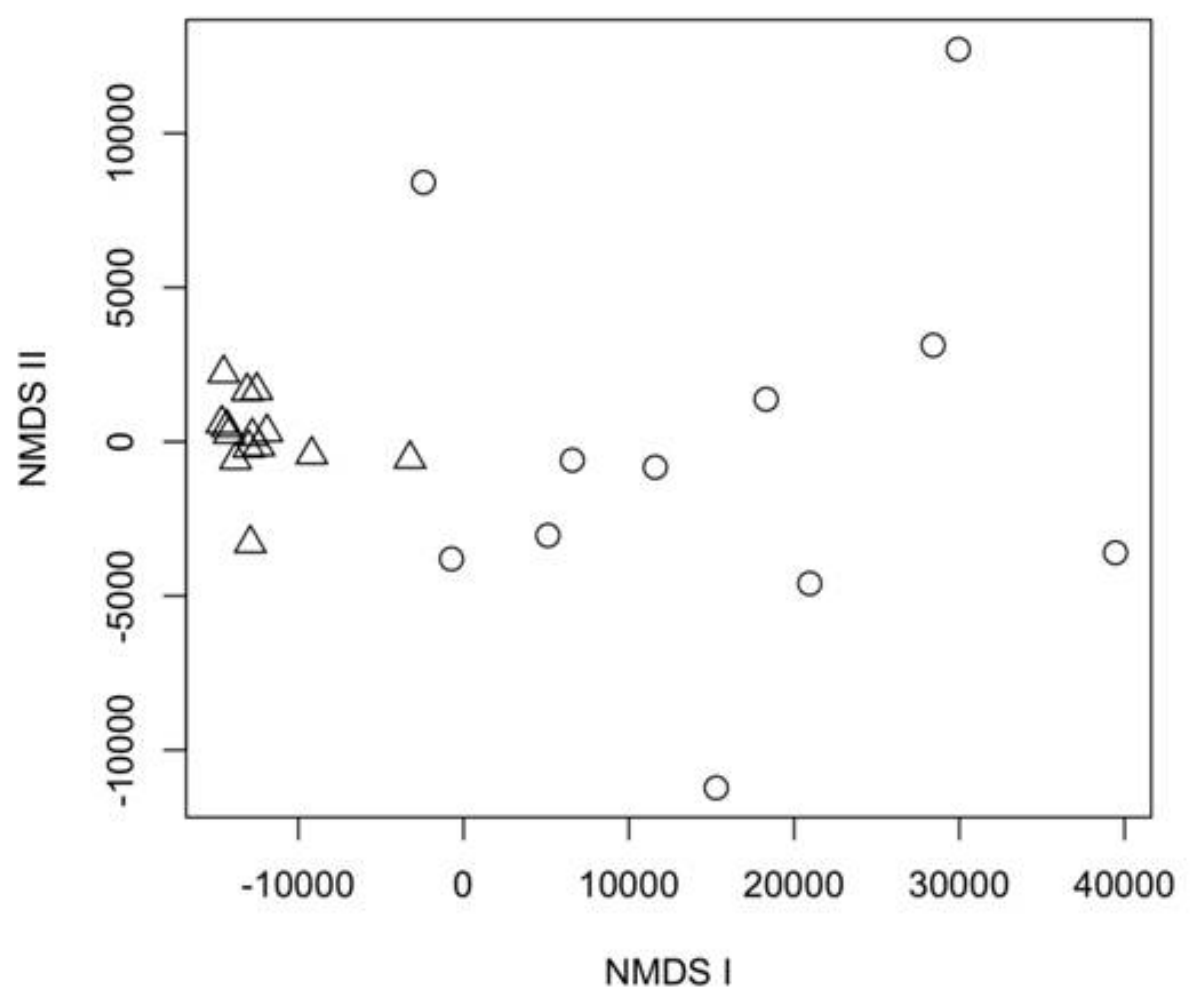


Figure 3.3 Springtails prefer female moss. The fraction of Ceratodon purpureus samples chosen by springtails (mean \pm se) in preference assays of a) male vs. female samples in Petri dishes ( $\mathrm{N}=24$ assays, 491 springtails counted); b) male vs. female samples in an olfactometer ( $\mathrm{N}=10$ assays, 276 springtails counted). ${ }^{* * *}$ Indicate means are significantly different $(\mathrm{P}<0.0001)$ within a graph.

a

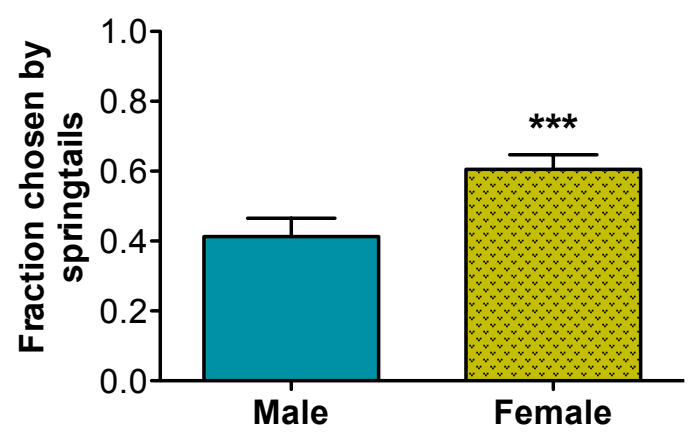

b

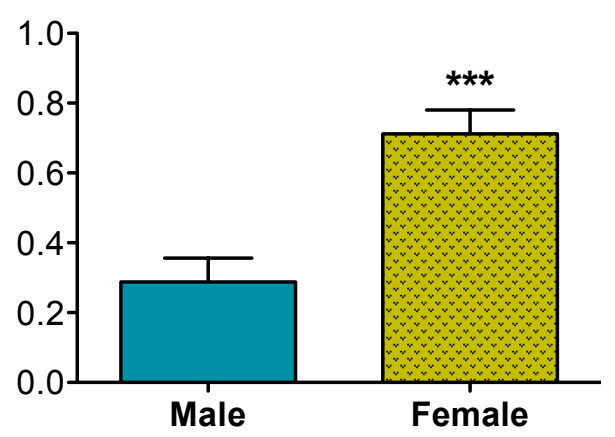


Figure 3.4 Springtails enhance fertilization in moss microcosms.

Fertilization success in Ceratodon purpureus and Bryum argenteum microcosms, measured as the fraction of microcosms that developed sporophytes (mean $\pm \mathrm{SE}$ ). The effect of a) springtail treatment (no springtails added vs. springtails added), b) water spray treatment (no spray vs. spray), and c) the interaction between these treatments on fertilization success. * Indicate means are significantly different $(\mathrm{P}<0.05)$ within a graph. $\mathrm{N}=108$ microcosms.

a

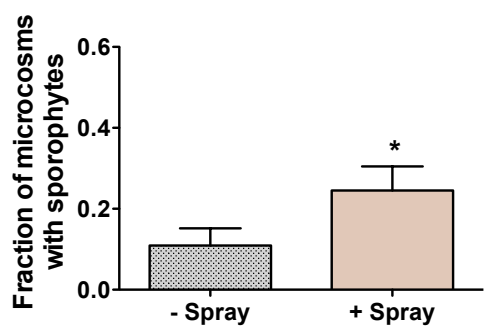

\section{b}

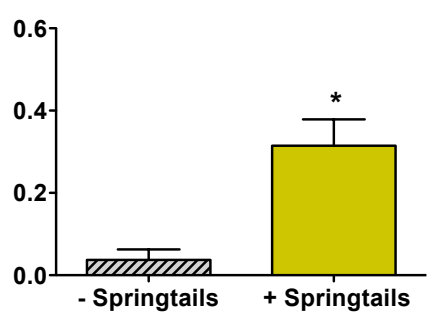

C

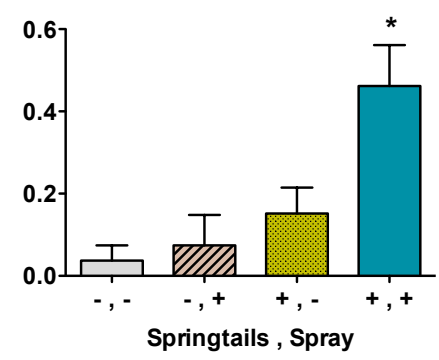




\title{
Chapter 4
}

\section{Community ecology of geothermal mosses: what drives biodiversity at temperature extremes?}

\begin{abstract}
Few organisms are capable of thriving at Earth's thermal extremes, yet those that do remain relatively unexplored, particularly ones engaged in complexmulti-trophic communities. While few vascular plants can survive in geothermal soils, non-vascular mosses are often found in some of the hottest regions of geothermal systems. We investigated the community ecology of geothermal mosses at Lassen Volcanic National Park, CA, in particular, asking what biotic and abiotic factors are driving this understudied, extreme ecosystem. We established study sites along an existing geothermal mosscommunity temperature gradient using the ubiquitous model moss species, Ceratodon purpureus as a focal species. We wanted to know if genetic variation exists among $C$. purpureus patches living in extreme temperatures, and if so, how does it relate to the structure and function of the mossassociated community? We profiled the invertebrate communities as well as fungal biomass, soil organic matter, and nutritive properties of the soil-moss complex across the geothermal gradient to obtain a snapshot of the moss ecosystem. We found significant trends in the biotic and abiotic factors
\end{abstract}


influencing moss-dominated communities along a geothermal gradient. We found that geothermal C. purpureus is not simply clonal, and that genetic variation exists primarily within localized populations, not among them, similar to findings of previous studies conducted in non-geothermal regions. The study site with the greatest moss genetic diversity fell at the mid-point of the temperature gradient and correlated positively with invertebrate diversity, fungal biomass and soil organic matter, presumably indicating a "hot spot" for community diversity and productivity. This study begins to disentangle and reconnect the multi-trophic factors driving geothermal moss communities. Our results indicate both intuitive and surprising ecological correlations, and present the intriguing prospect that the universal mossmicroarthropod relationship may be affecting moss diversity.

Keywords: Ceratodon purpureus, community ecology, geothermal, microarthropods, moss 


\section{Introduction}

Mosses are abundant worldwide (Shaw \& Goffinet, 2000) and found in mesic, urban, and extreme environments living on, under, within, among, and sometimes fairly isolated from other organisms. Most taxa do not thrive across the circumference of terrestrial Earth nor span the poles, yet mosses are capable of covering these abiotic ranges, making them exceptionally successful organisms (Glime, 2007). Mosses regularly colonize some of the coldest regions (Arft, 1999, Bramley-Alves et al., 2014, Longton, 1988, Ochyra et al., 2008) and some of the hottest regions worldwide (Bargagli et al., 1996, Bonini et al., 2005, Convey \& Lewis Smith, 2006, Elmarsdottir et al., 2003, Glime \& Hong, 1997, Kappen \& Smith, 1980, Smith, 1981). Throughout their ranges, mosses are responsible for significant global primary productivity and biogeochemical cycling (DeLuca et al., 2002, Kip et al., 2010, Lindo \& Gonzalez, 2010, Turetsky, 2003, Turetsky et al., 2012) and execute functional roles in establishing, regulating and moderating plant-based, multi-trophic communities in both mesic and extreme environments (Glime, 2007, Gornall et al., 2007, Jassey et al., 2013, Ohlson et al., 2001, Turetsky et al., 2012, Van Der Wal \& Brooker, 2004). These studies reveal that the often-overlooked mosses facilitate and regulate both local and global processes. Still many questions remain concerning aspects of the functional biology and dynamics of these unique communities, particularly those living 
at thermal extremes where one might expect the system itself to be relatively simplified, since few organisms can survive such conditions (Sunday et al., 2011).

Assessments of whole community dynamics at high temperatures are often focused on microbial organisms. Researchers have assessed microbial communities at hydrothermal vents (Grassle, 1986, Reysenbach \& Shock, 2002), and in geothermal sites cyanobacterial mats and isolated bacterial communities have been characterized (Klatt et al., 2011, Walker et al., 2005, Ward et al., 1998). Yet, many geothermal regions are comprised of complex multi-trophic communities, and likely due to the fact that relatively scant grasses and vascular plants even survive in geothermal regions (Stout \& AlNiemi, 2002), studies on these plants in geothermal systems remain sparse. Grasses growing in geothermal soils often have endophytic and mycorrhizal associations helping to enable root tolerances to high temperatures (Appoloni et al., 2008, Marquez et al., 2007, Rodriguez et al., 2008). Other studies have suggested that mosses living on geothermal soils may also aid in ameliorating thermal stress to grasses (Tercek \& Whitbeck, 2004) as they have in other stressful environments including serpentine (Freestone, 2006) and Antarctic soils (Casanova-Katny \& Cavieres, 2012).

Nonvascular communities are often the dominant plants in geothermal regions, and with limited exception, have been grossly understudied. Such exceptions include reports of geothermal moss community composition (Smith, 
1981, Smith, 2005), abiotic parameters (Chiarucci et al., 2008), and

relationships to soil characteristics and vascular plants (Glime \& Hong, 1997, Tercek \& Whitbeck, 2004). Mosses living at thermal extremes often have low viable sporophyte yields, indicating that there are significant limitations to reproduction for mosses living at high temperatures (Convey \& Smith, 1993, Eppley et al., 2011, Rosenstiel \& Eppley, 2009, Skotnicki et al., 2002, Stark et al., 2000). Yet, dispersal and genetic diversity of mosses worldwide have been found to be more complex than expected (Clarke et al., 2009, McDaniel \& Shaw, 2005, Skotnicki et al., 2004, Szovenyi et al., 2009, Van der Velde \& Bijlsma, 2000, Van der Velde et al., 2001b) and studies show that sexual reproduction in mosses can be partially facilitated by the microarthropod communities that ubiquitously thrive in moss patches (Cronberg et al., 2006, Gibson \& Miller-Brown, 1927, Rosenstiel et al., 2012).

Studies of the invertebrate communities living in moss patches typically identify the individuals (Bolter et al., 1997, Convey, 1996, Lindo \& Winchester, 2006, Usher \& Booth, 1984), while few other studies have worked to assess the entire moss-invertebrate community complex as a functional system (Davis, 1981, Gonzalez et al., 1998, Lindo \& Gonzalez, 2010). More recently, moss has been employed as scaffolding by which to assess and scale-up metacommunity dynamics (Chisholm et al., 2011, Gonzalez et al., 1998, Leibold et al., 2004, Srivastava et al., 2004, Staddon et al., 2010). These studies elegantly involve the moss-microarthropod complex 
as a model and a platform to test questions about concepts such as connectivity, nutrient cycling and patch dynamics (Chisholm et al., 2011, Staddon et al., 2010), but the mosses are often extracted from their original community and used to simulate a community structure. When examined in this manner it may be impossible to know what may actually be driving the systems in their natural states.

A few studies have profiled geothermal moss canopy-level interactions in the field (Elmarsdottir et al., 2003, Fraser et al., 2014) and to our knowledge, no studies have attempted to assess the drivers of geothermal moss-based community patterns and biodiversity, nor how genetic diversity of the foundation species may influence the functional ecology of the system. Extreme thermal environments often have simplified, less stable communities compared to more mesic environments (Tilman \& El Haddi, 1992, Wall \& Virginia, 1999). A shift in conditions could limit an organism's ability to survive and adapt if there is less genetic variation on the landscape. Genetic diversity of individuals in a community can influence not only fitness and diversity but also effect organisms of multiple trophic levels, in turn impacting the entire community (Hughes et al., 2008, Whitham et al., 2006). Whether there is low genetic or bio-diversity in moss-dominated geothermal communities remains unknown.

In this study we use concepts from functional community ecology to investigate the key players in a moss-dominated landscape along a 
geothermal gradient, in situ at Lassen Volcanic National Park (LVNP), California, USA. Incorporating an existing environmental gradient into community studies can be key to understanding the functional ecology (McGill et al., 2006). A small central group of mosses live in the hottest regions of LVNP (Eppley et al., 2011, Showers, 1982), one of which, Ceratodon purpureus is a model moss species whose distribution spans from the Arctic to the Antarctic (Crum, 1973). We used the occurrence of this common moss, growing along a geothermal gradient as a foundation by which to begin profiling the biotic and abiotic factors that drive the moss-dominated geothermal system balanced at Earth's thermal extremes. We predicted that a thermal gradient would dictate the community-wide moss diversity and function, and ultimately limit both at the hottest thermal extremes.

\section{Materials and Methods}

\section{Study site and experimental design.}

This study was conducted in the southwest region of Lassen Volcanic National Park (LVNP), where we have extensively surveyed for bryophytes around geothermal features (Eppley et al. 2011). Surveys in 2006 found bryophyte communities at Boiling Springs Lake (BSL), mean elevation of 1799 meters) and Devil's Kitchen (mean elevation of 1854 meters), but not at eight additional geothermal regions in the park where surveying was feasible. 
The chemical composition of the springs and geological system of the area have been well-documented (Muffler et al., 1983, Siering et al., 2006, Snyder, 2005, Thompson, 1982, Thompson, 1983). At the elevation of the springs, nongeothermal areas are primarily mixed coniferous forests of Jeffery Pine (Pinus Jeffreyi) and White fir (Abies concolour), with few bryophytes (Polytrichum juniperinum) in forest gaps.

Surrounding BSL, N 40.43404; W 121.39697), we previously identified five primary bryophyte species growing in geothermally-heated soils: Aulacomnium palustre (Hedw.) Schwägr., Campylopus introflexus (Hedw.) Brid., Ceratodon purpureus (Hedw.) Brid., Ptychostomum pseudotriquetrum (Hedw.) J.R. Spence \& H.P. Ramsay, and Bucklandiella affinis (Schleich. ex Weber \& Mohr, Ochyra et al., 2008). Angiosperms associated with the geothermal areas at LVNP include Dichanthelium acuminatum (Sw.) Gould \& C.A. Clark, Agrostis scabra Willd, and Mimulus guttatus DC.

In October 2012, five geothermal sites were established within $100 \mathrm{~m}$ of BSL predicated on the sufficient presence of our target species, Ceratodon purpureus occurring across a range of at least three meters in diameter. Morning rhizoid temperatures ranged from $13^{\circ} \mathrm{C}-50^{\circ} \mathrm{C}$, indicating a thermal gradient. The dominant moss species at each site other than C. purpureus was as follows: Site 1: B. affinis; Site 2: A. palustre; Site 3: C. introflexus; Site 4, A. paulstre, and Site 5, A. paulstre and P. pseudotriquetrum. All sites had zero to three of the above listed angiosperm species within the sites, 
typically growing out of the moss beds if present. The angiosperm occupied less percent cover than the mosses at all sites.

A central location of $C$. purpureus was determined and marked at each site, and three sequential rings around the center point were flagged (0.5, 1.0, $2.0 \mathrm{~m}$ from center) with four locations on each ring identified for the presence of $C$. purpureus, resulting in 13 locations including the central point per site. All sites are geothermal, and had varying percent cover of C. purpureus, ranging from $\sim 10 \%$ to $80 \%$. The contiguous patches of $C$. purpureus varied greatly in size (ranging approx. $1 \mathrm{~cm}$ to $50 \mathrm{~cm}$ diameter) both between and among sites.

\section{Temperature and relative humidity.}

To characterize our sites initially for temperature, in the fall of 2012 we collected canopy-level temperatures and lux values using data loggers (Pendant; Onset Computer Corporation, Bourne, MA) logging for every five minutes for eight consecutive days. Additionally, midday (MD) temperatures at moss rhizoid level (just below the photosynthetic green canopy where the moss root-like structure, the rhizoid, attaches to substrate) were recorded at each of 13 C. purpureus sub-sites using K-type thermocouple probes with a high-accuracy digital thermometer with a two-point temperature averaging function (HH-23A, Omega Engineering Inc., Stamford, CT), at all five sites prior to collecting moss tissue. 
To obtain the full moss-community temperature horizon, in the spring of 2013, temperatures of the central patch of C. purpureus of each site were recorded at 1) soil level (2 $\mathrm{cm}$ below rhizoids), 2) rhizoid level, and 3) canopy level ( $1 \mathrm{~cm}$ into the green canopy). Temperatures were recorded both before sunrise, predawn (PD) and just after sites were exposed to morning sun (AM). All temperatures were collected using K-type thermocouple probes with a high-accuracy digital thermometer with a two-point temperature averaging function (HH-23A, Omega Engineering Inc., Stamford, CT)

Data loggers to track percent relative humidity $(\mathrm{RH})$ were placed in each site at canopy-level (HOBO Pro v2; Onset Computer Corporation, Bourne, MA) and recorded for eight days in October 2012.

\section{Moss collection.}

We based our study on C. purpureus, and we did so for a number of reasons. First, it is a cosmopolitan moss (Crum, 1973) that widely occurs in geothermal systems (Convey et al., 2000). Further, it is a dioicous moss (having separate sexes, thereby unable to self-fertilize) that infrequently reproduces sexually in thermal regions (Eppley et al., 2011), although it is relatively abundant and is a known stress-tolerator (Cove et al., 2006) with typically frequent sporophyte production (McDaniel, 2005). The species is well studied from a population genetics perspective (McDaniel \& Shaw, 2005, Shaw \& Gaughan, 1993), a physiological perspective (e.g. Clarke \& Robinson 
2008, Turnbull et al. 2009) and from sexual and mating systems perspectives (McDaniel et al., 2013b, Rosenstiel et al., 2012). In addition, the C. purpureus genome of both males and females have been recently sequenced (pers comms SF McDaniel) and several primers for microsatellite regions in autosomal regions of the genome have been developed and tested (Clarke et al., 2008).

At all 13 locations at each site, C. purpureus was field identified and a small portion was collected using forceps into vouchers of $\sim 1 \mathrm{~cm}$ diameter and placed into paper envelopes. Specimens were immediately transferred into an airtight container over anhydrous desiccant (Drierite, W.A. Hammond Drierite Company LTD) and were verified for species identification by compound microscopy (Leica MZ) in the laboratory at Portland State University (PSU).

\section{Genetic variation.}

To assess fine-scale genetic variation among geothermal C. purpureus populations (Selkoe \& Toonen, 2006), genomic DNA of individual $C$. purpureus gametophytes from each location was extracted and analyzed using microsatellites to attempt to assess fine-scale genetic variation among geothermal C. purpureus populations (Selkoe \& Toonen, 2006). DNA extractions were conducted using a high-throughput system for rapid extraction and amplification of genomic DNA by use of Plant REDExtract-NAmp kit components (Sigma-Aldrich, USA) and using a modified version of 
manufacturer protocols. To extract DNA, single gametophytes were placed, using sterilized forceps, into microtubes with $20 \mu \mathrm{L}$ of extraction solution (Sigma-Aldrich) and briefly disrupted the tissue using pipette tips. Samples were incubated (Applied Biosystems Thermocycler 2720) at $65^{\circ} \mathrm{C}$ for 10 minutes (min), followed by $10 \mathrm{~min}$ at $95^{\circ} \mathrm{C}$ and then held at $4^{\circ} \mathrm{C} .20 \mu \mathrm{L}$ of neutralization solution was added to the incubated samples, vortexed briefly and DNA was used immediately for amplification or kept at $4^{\circ} \mathrm{C}$ until use with REDExtract-N-Amp PCR Ready Mix. We used 5' forward (FAM labeled) and reverse primers (Eurofins OPERON) designed and described in Clarke et al. 2008 (Clarke et al., 2008). Of five previously published markers, four reliably amplified the desired DNA regions.

For a $10 \mu \mathrm{L}$ reaction, $5 \mu \mathrm{L}$ REDE-mix, $0.5 \mu \mathrm{L}$ of both forward and reverse primers $(0.5 \mu \mathrm{M}$ final concentration), $3 \mu \mathrm{L}$ of $\mathrm{RNAse}$ free sterile water and $1 \mu \mathrm{L}$ of DNA template. DNA was amplified with Applied Biosystems Thermocycler 2720 with conditions as follows: $10 \mathrm{~min}$ at $94^{\circ} \mathrm{C}$, followed by 35 cycles of: $30 \mathrm{~s}$ at $94^{\circ} \mathrm{C}, 30 \mathrm{~s}$ at $57^{\circ} \mathrm{C}, 45 \mathrm{~s}$ of $72^{\circ} \mathrm{C}$, and followed by $10 \mathrm{~min}$ at $72^{\circ} \mathrm{C}$ and held at $\mathrm{t} 4^{\circ} \mathrm{C}$ until use or storage at $4^{\circ} \mathrm{C}$. For CEPU 111 the hold at $72^{\circ} \mathrm{C}$ was extended to 20 min to allow ample time for the final addition of the adenosine nucleotide as preliminary analyses indicated the extension time was necessary to confidently identify fragment lengths for this particular region. PCR products were tested for DNA amplification using 1.5\% agarose gel in 1 x TAE Buffer. Gels were run at $90 \mathrm{v}$ for $50 \mathrm{~min}$, and visualized on a 
Typhoon Trio Imager (Amersham Biosciences) to verify primer adequacy (by FAM fluorescence) and then verified by ethidium bromide or SYBR green staining and then visualized and photographed using a transilluminator. Samples were kept at $4^{\circ} \mathrm{C}$ until $1 \mu \mathrm{L}$ per sample was sent to the Center for Genome Research and Biocomputing Core Labs at Oregon State University (Corvallis, OR, USA) for sequencing via an AB 3730 capillary DNA sequencer to resolve fluorescently labeled DNA fragments for genotyping applications. Rox40 internal standard was used to score alleles (bp) using Peak Scanner software.

\section{Microarthropod collection.}

To determine how microarthropod communities correlate with temperature and moss patch characters, microarthropod communities were sampled from each site using two complimentary collection methods: pitfall traps and extraction funnels (Briggs 1961, Edwards 1991) on two occasions: first in the fall (October) of 2012 and again in the spring (June) of 2013. On both occasions pitfall trappings were conducted in situ using round hard-plastic cups (5.5 cm diameter, $6 \mathrm{~cm}$ deep) inserted into the ground at three random locations within the $3 \mathrm{~m}$ radius of each site with the lip of the cup flush with moss rhizoid level (Spence \& Niemela, 1994). The cups were filled to $3 / 4$ full with non-toxic propylene glycol (Weeks \& McIntyre, 1997), which effectively preserves and traps passing invertebrates but has a slow evaporation rate 
which is crucial in hot soils. All pitfall traps were left in the sites for 48 hours.

Immediately before leaving LVNP, $5.8 \mathrm{~cm}$ moss/rhizoid/soil complex cores (inserted $\sim 1 \mathrm{~cm}$ into the soil-rhizoid complex) were extracted from three random locations at each of five sites for microarthropod collections. Cores were collected into plastic bags and kept in a cooler until returning PSU (within 16 hours of collection) for extraction by modified Berlese funnels (Macfadyen, 1953) via a humidity and light gradient (Andrew et al., 2003). The cores ensure for controlled patch sizes and insect densities with sound replicates (Borcard \& Legendre, 1994). All three cores from each of five sites were pooled for invertebrate extraction and placed in collapsible canvas funnels (BioEquip, CA). Funnels were illuminated and heated by 15 watt incandescent light bulbs for 48 hours. Extracted invertebrates were collected into a dilute ethanol solution ( $80 \%$ ethanol). All samples from both sampling methods were sorted, counted, and identified into functional groups by site under a stereomicroscope (Leica MZ).

\section{Fungal Biomass Estimation.}

In June 2013, we collected moss canopy cores to quantify ergosterol content (a sterol found in fungi) to establish an estimate of living fungal biomass in our moss canopies. Three $3.8 \mathrm{~cm}$ diameter cores were taken from three locations at each site immediately before leaving LVNP and stored in a cooler 
until returning to PSU. Cores were then were binned by site, and whole gametophytes were washed thoroughly in deionized water to remove soil and coarse debris and lyophilized. 100-200 mg of lyophilized tissue was placed in $2 \mathrm{ml}$ microcentrifuge tubes with a zirconium bead and ground to a fine powder using a bead beater at $2500 \mathrm{RPM}$ for 40 seconds. To extract ergosterol from powdered tissues we used modified methods of (Dahlman et al., 2002). Samples were suspended in $1 \mathrm{ml} \mathrm{MeOH}$, agitated in an orbital shaker for $1 \mathrm{~h}$ at 320 RPM in darkness, and subsequently allowed to precipitate overnight at $4^{\circ} \mathrm{C}$. Extractions were then centrifuged for $1 \mathrm{hr}$ at $14,000 \mathrm{RPM}$ and $4^{\circ} \mathrm{C}$. Following centrifugation, the supernatant from each extraction was transferred to a new $2 \mathrm{ml}$ microcentrifuge tube and centrifuged for an additional $10 \mathrm{~m}$ at $140,000 \mathrm{RPM}$ and $4^{\circ} \mathrm{C}$. The supernatants from the secondary centrifugation were filtered through $0.2 \mu \mathrm{m}$ acrodiscs into amber autoanalyzer vials and stored at $4{ }^{\circ} \mathrm{C}$ until HPLC analysis. Extractions were analyzed following (Davey et al., 2012) Davey et al. 2012 on an 1100 Series HPLC (Agilent Technologies, Waldbronn, Germany).

\section{Carbon and Nitrogen Analyses.}

To determine how total vegetative and soil carbon and nitrogen correlate with temperature, total nitrogen and carbon content of dried and ground soil and moss tissue were determined via combustion with an elemental analyzer (Thermo-Fisher Scientific, Flash Series EA1112, CE Elantech, Lakewood, 
NJ) using the manufacturer's NC soils configuration and protocols. Moss cores collected for funnel microarthropod extractions from the spring 2013 field campaign were collected for use in multiple assays. After $48 \mathrm{~h}$ of invertebrate extraction the moss and soil was completely ambient-air dried. The moss cores were then carefully sorted to separate soil and debris from moss tissue. All separated materials were placed in paper bags and dried in a drying oven at $60^{\circ} \mathrm{C}$ for $48 \mathrm{~h}$. Dried soil was put through a fine sieve to remove debris and both soil and moss tissue were ground to the consistency of a homogeneous fine powder using individual sterilized ceramic mortars and pestles and immediately transferred into envelopes to be kept in air-tight containers containing anhydrous desiccant (Drierite, W.A. Hammond Drierite Company LTD) until processing. For moss tissue analysis, between 5 - $6 \mathrm{mg}$ of dried and ground tissue was used, and $12-13 \mathrm{mg}$ of dried soil was used for soil analysis, five replicates from each site were processed for both.

\section{Analysis of soil organic matter and soil pH.}

The remaining dry, ground soil from each core collected in June 2013 was used for analysis of $\mathrm{pH}$ and the percent soil organic matter (SOM). Three replicates of prepared soil from each site were dried, weighed, placed in crucibles where site replicates were randomized inside of an electric furnace (Cress Mfg. Co.) and fired to $550^{\circ} \mathrm{C}$ to combust organic matter. SOM was determined using the loss on ignition (LOI) method (Heiri et al., 2001). To 
test soil $\mathrm{pH}, 30 \mathrm{mg}$ of soil from each site was mixed thoroughly with $30 \mathrm{~mL}$ of deionized water in glass beakers and allowed to settle for one hour. $\mathrm{pH}$ and temperature of the supernatant was quantified using a standardized $\mathrm{pH}$ meter (Fisher Scientific). Seven $\mathrm{pH}$ readings of each site's soil were recorded.

\section{Data Analysis.}

Two-way ANOVA were performed on our between and within site temperature data from 2013 along a vertical gradient to test variance between and among sites and horizons. Mixed model ANOVA was performed on invertebrate data to assess if there were significant differences between sampling method (pitfall trap or funnel), sites and season (spring or fall). The Gini-Simpson Diversity Index, a biological method for calculating within population diversity (Rao, 1982) was determined for invertebrate group diversity at each site.

The partitioning of genetic variance within and among spatially segregated populations containing polymorphisms at our single loci was determined using Analysis of Molecular Variance (AMOVA) (Excoffier et al., 1992), yielding the PhiPT statistic, an analogue of $\mathrm{F}_{\mathrm{ST}}$ (Weir \& Cockerham, 1984) using GenALEx 6.5 software (Peakall \& Smouse, 2012, Peakall \& Smouse, 2006). The sum of squares within population (SSWP) (Michalakis \& Excoffier, 1996) statistic was subsequently used to infer C. purpureus diversity between sites. 
Ergosterol content was analyzed following (Davey et al., 2012) on an 1100 Series HPLC (Agilent Technologies, Waldbronn, Germany). SOM content was calculated using the LOI method (Heiri et al. 2001). Soil and tissue nitrogen values were integrated with a standard curve of known $\mathrm{C}$ and $\mathrm{N}$ content (aspartic acid) and calculated using K-type calibration for nonlinear values as recommended and carried out on manufacturer's software (Eager Xperience ver. 1.2, 2011).

For full community-level analyses, each site's average value of each collected variable was compiled into a data matrix. A correlation matrix was generated from the data matrix and principle component analyses (PCA) were performed on scaled correlations. Not all variables contributed to explain the variance in the dataset; therefore, those that contributed poorly to the model were systematically eliminated by assessing the variables with the greatest contribution to the lesser PCs (Jolliffe, 2005), until the first two PCs accounted for the majority of the variance in the data set. The variables that did not significantly contribute to the model (or were auto-correlated (i.e. rhizoid and canopy temperatures) were systematically dropped from the PCA included: soil $\mathrm{pH}$, rhizoid temperature, percent tissue $\mathrm{N}$, and moss tissue C:N. Non-oribatid mites, and all other invertebrate taxa auto-correlated with Invertebrate Diversity so were also dropped from the model. ANOVA were run on rhizoid temperatures, relative humidity, invertebrate numbers, soil 
and percent tissue $\mathrm{N}, \mathrm{C}: \mathrm{N}$, ergosterol content, $\mathrm{SOM}$ and $\mathrm{pH}$ values to determine variances between and within sites.

Community-level PC analyses were conducted using R Statistical Package (R Development Core Team, 2006). All other analyses unless otherwise described above were conducted using JMP 10.0.2 (SAS Institute, 2012).

\section{Results}

\section{Temperature and relative humidity of five sites}

In situ temperature measurements revealed that our sites follow a thermal gradient where mean canopy site temperatures deviate significantly from one another (Fig. 4.1a; $\mathrm{F}=901.6 ; \mathrm{P}<0.0001 ; \mathrm{N}=2343$ ) with mean fall temperatures ranging from $7.9^{\circ} \mathrm{C} \pm 0.04 \mathrm{SE}$ to $12.2^{\circ} \mathrm{C} \pm 0.10 \mathrm{SE}$ for the coolest and hottest sites respectively. We found the five sites to follow the same thermal gradient at rhizoid-level. Averaged point measurements of each site's subsites reveal fall rhizoid temperature means to be significantly different between sites (Fig. 4.1b; $\mathrm{F}=29.07 ; \mathrm{P}<0.0001 ; \mathrm{N}=13$ ), with means ranging from $15.5^{\circ} \mathrm{C} \pm 0.43 \mathrm{SE}$ to $33.4^{\circ} \mathrm{C} \pm 1.80 \mathrm{SE}$ at our coolest to hottest sites $(1,5)$ respectively. There are also significant differences between percent relative humidity between the five sites (Fig 4.1c; $\mathrm{F}=2185, \mathrm{P}<0.0001 ; \mathrm{N}=2335$ ) with the lowest mean relative humidity at the coolest site (Site 1 ) at $38.3 \% \pm 0.27$ SE and the highest at the hottest site (Site 5) with a mean of $86.6 \% \pm 0.08$. 
The relative humidity at these LVNP sites is commonly a product of the geothermal vents running along the soils or from the high evaporation rates of BSL. Lux values (light level; lumen per square meter) were also different between sites (Fig. 4.1d; F=136.8, $\mathrm{P}<0.0001 ; \mathrm{N}=4670$ for all sites except Site 3, $\mathrm{N}=2335$.) Again, Site 1 had the lowest mean value of $1896 \pm 107.2)$ and the highest light levels at Site 5 the highest $(5315 \pm 140.1)$. We want to note that during temperature and relative humidity data collections in the fall of 2012, an early snowstorm fell on our sites, we collected the data loggers after snowmelt (at geothermal sites) and speculate that mean temperatures in October are typically slightly higher than those reported here.

We also found significant differences in temperature along a vertical horizon between shallow soil, rhizoid and canopy levels in the sites during the spring of 2013. We found these differences to be present both in the morning pre-dawn (PD) and after dawn (AM) when each site was newly in full sun-exposure (Fig. 4.2). The majority of significant differences between sites and locations fell between soil and canopy temperatures, both PD and AM (for ANOVA statistics see Table 4.1). Our soil temperatures range from a low of $32.1^{\circ} \mathrm{C}$ to a high of $51.3^{\circ} \mathrm{C}$, rhizoid temperatures from $19.9^{\circ} \mathrm{C}$ to $41.5^{\circ} \mathrm{C}$, and canopy temperatures from $14.5^{\circ} \mathrm{C}$ to $36.5^{\circ} \mathrm{C}$, representative lows and highs were recorded in Sites 1 and 4, respectively.

\section{Moss variation}


We successfully amplified four microsatellite regions for at least five individuals from each site, up to 24 individuals per site. One somatic region was polymorphic, and this particular marker has been reported as the most polymorphic in worldwide C. purpureus diversity assessments (CEPU105, for details, see Clarke 2009). Of 99 successfully amplified and identified individuals $(20,18,27,15$, and 18 from each of Sites $1-5$, respectively) we found that $13 \%$ of genetic variation at this loci is found among populations, while $83 \%$ is within populations ( $\mathrm{PhiPT}=0.135, \mathrm{P}=0.001$ ). There was no correlation between number of samples amplified and identified and diversity. These data are consistent with other population genetic analyses of $C$. purpureus where the majority of variation is found within populations but not among (McDaniel \& Shaw, 2005). The estimated within population variation of the haploid population's sum of squares within population (SSWP) diversity statistics were: $6.85,6.72,9.70,7.90,6.20$ for Sites 1-5 respectively (Fig 4.5). In the other three of the four amplified microsatellite regions we saw no variation in allele size. We use the SSWP values from our polymorphic allele for representation of $C$. purpureus variation within our five sites in subsequent community analyses.

\section{Microarthropods}

Thousands of geothermal invertebrates (6113 total; 2860 in fall 2012; and 3203 in spring 2013) were successfully collected using the two collection 
methods. Invertebrates were identified to major orders or sub-orders. Each site varied in invertebrate abundance by site. The total number of invertebrates collected in October was: $59,818,1580,326$, and 77 , and in June was: 299, 602, 485, 941, and 876 respectively from Sites 1-5. (Fig. 4.4). Both collection methods successfully yielded invertebrates, with the pitfall trap overall invertebrate numbers higher than the funnel extraction method (3763 and 2350 respectively), although one method did not consistently yield more or less invertebrates among sites (Fig. 4.4) and the mixed model ANOVA reveals no significant differences in invertebrate abundances collected between or among site, method, time, or the interactions among them $(\mathrm{F}$ ratio $=0.98, \mathrm{P}=0.57)$. The species diversity of invertebrate groups in each site was calculated using the Gini-Simpson Index (Jost, 2006) with higher values representing greater invertebrate diversity. Gini-Simpson diversity values fall steadily with increasing temperature: $0.75,0.63,0.43$, 0.32 and 0.16 respectively (Fig. 4.3a).

The dominant microarthropods in our sites at LVNP were Acari and in particular, mites of the suborder Oribatida ( $47 \%$ of total), followed by Collembola species (37\%), non-oribatid mites (these mites were identified as either Prostigmata, Mesostigmata or Astigmata; 9\%), followed by other Arachnida, Coleoptera, Diptera, Hymenoptera and worms represented 1\% of the total invertebrate abundance each. We present the relative abundances 
of the invertebrate communities by major functional group at each of our five geothermal sites at LVNP (Fig. 4.3b).

\section{Community parameters}

Soil $\mathrm{pH}$, percent soil and tissue nitrogen, and moss tissue C:N, and soil organic matter all vary significantly between sites (see Table 4.3 for values and ANOVA statistics). Mean ergosterol (ug/g tissue) per site is reported in individual numbers as follows for Sites 1-5 respectively as samples were binned: $57.35,48.56,61.23,48.44$, and $23.89 \mathrm{ug} / \mathrm{g}$ dry tissue. Site 5 (the hottest site) had the lowest values of ergosterol (23.89 ug/g tissue), soil nitrogen $(0.07 \%)$, soil $\mathrm{pH}(4.7)$, and $\mathrm{SOM}\left(\mathrm{LOI}_{550}=0.25\right)$, as well as the lowest C.purpureus within population variation (SSWP of 6.2) Interestingly the site with the highest values for most parameters was Site 3. Site 3 maintained the greatest ergosterol levels (61.23 ug/g dry tissue), percent soil and tissue nitrogen $(0.65 \%$ and $0.84 \%$, respectively $)$ and $\mathrm{SOM}\left(\mathrm{LOI}_{550}=1.04\right)$. Site three also hosted the greatest within population variation of C. purpureus (SSWP), which correlated with SOM (94\%) and relative abundance of Collembola (74\%) and the highest number of overall invertebrates (Fig. 4.4). Relative abundance of lesser represented invertebrates as well as non-oribatid mites was greatest at Site 1, our coolest site where non-oribatids are significantly negatively correlated with soil temperature (-96\%), but positively with overall invertebrate diversity (89\%). Oribatid mites and high temperatures 
correlated positively (90\% with canopy temperatures), but inversely with fungal biomass (ergosterol, -84\%). A cluster analysis on the data matrix was executed (Ward method) and revealed that Sites 1 and 2 cluster, as do 4 and 5, with Site 3 also clustering with Sites 1-2 but forming a separate branch from the initial node, thus even with all variables considered, the cluster analysis and PCA both track the basic geothermal temperature gradient (Fig. 4.1). The aforementioned community relationships are illustrated in the principle component analysis where $88.7 \%$ of the variance in the data set is explained by the first two principle components (Fig $4.6 ; 51.4 \%$ by the first PC, and $37.3 \%$ by the second PC; for PC loadings see Table 4.4).

\section{Discussion}

We hypothesized that thermal extremes would limit the community-wide diversity in our study sites at LVNP, and we found that a temperature gradient dictates much of the community differences among our sites (Fig. 4.1a). Even though our geothermal system is extremely dynamic (Fig. 4.2) and mostly comprised by geothermal moss, our sites were quite divergent from one another in most measures. The sites that are more similar to one another are not the closest in physical location, moss composition, nor in distance from BSL, but instead in temperature indicating that even in a geothermal region where life is limited by thermal extremes, a physical gradient exists that at least partially defines the rest of the community. The 
findings that most directly support our temperature-gradient-driven community hypothesis are the invertebrate diversity results (Gini-Simpson Index). The highest invertebrate diversity is at the coolest site and declines sharply as site temperature increases, rendering the hottest site with the lowest invertebrate diversity (Fig. 4.4a). The two hottest site's invertebrate populations were dominated by oribatid mites (Site 5: $91 \%$ and Site 4: $82 \%$;

Fig. 4.4b), which are often the most common moss-microarthropod in patches worldwide from the Antarctic, to urban streets to geothermal sites (Convey et al., 2002, Lindo \& Winchester, 2006, Usher \& Booth, 1984), and our data would imply are the most stress tolerant. Interestingly, the temperature gradient did not correlate with gross invertebrate abundance (Fig. 4.3), which did not follow any discernable pattern other than tracking the mean relative humidity and light levels of the sites (Fig. 4.1c) with the exception of Site 5, which has high abiotic values, but lower overall invertebrate abundance. In fact, the coolest site, Site 1, although having the most invertebrate diversity, it had the least in abundance. Thus, our hypothesis was partially true in that the invertebrate diversity is correlated to temperature, but abundance is not.

In most systems relative humidity and light levels would be inversely related, but in our sites they are positively correlated (Fig. 4.1c,d), due to BSL near-constant evaporation rates and geothermal vents running throughout the sites, further solidifying this system as different from other 
extreme environments and uniquely suited to assess community structure drivers. Another interesting aspect of these data is that oribatid mite dominance is negatively related to ergosterol levels (-0.84; Fig. 4.6) which we can interpret to mean one of two things: either fungi is not as prevalent in the hottest sites, contrary to what has been found in geothermal vascular plant associations (Rodriguez et al., 2008), or that the mites (often fungivores) are abundant enough that they keep the fungal populations down as a function of their grazing. A more in-depth analysis of the fungal community, feeding guilds and preferences of oribatids and other invertebrates, as well as isotopic labeling of $\mathrm{N}$ and $\mathrm{C}$ to trace nutrient pathways could produce valuable insight into this relationship.

Perhaps the most interesting findings of our study occur at the site right in the middle of the thermal gradient. Other than in invertebrate diversity, which is greatest at Sites 1 and 2 , Site 3 , the median site, appears to be the biological "hot-spot" of the geothermal moss-communities surveyed. Site 3 has is dominated by an invasive extremely stress tolerant moss, $C$. introflexus, has the highest fungal and microbial loads (inferred from ergosterol content and SOM), as well as high levels of moss tissue nitrogen and carbon, and soil nitrogen (Table 4.3; Fig. 4.6). Further, the site has the highest numbers of overall invertebrates whose activities are thought to contribute to moss's role in facilitating microbial activity and increasing net nutrient cycling (Jassey et al., 2013, Turetsky, 2003), inducing higher levels 
of organic matter and decomposition rates, thus nutrients are likely more available in biologically active canopies. The invertebrate abundance at the site is mostly comprised of Collembola (76\%; Fig 4.4b). Collembola are ubiquitous moss-dwelling microarthropods that typically feed on microbial life, are key in soil detrital and nutrient cycles, particularly in increased $\mathrm{N}$ availability in the matrix (Hopkin, 1997, Osler \& Sommerkorn, 2007, Turetsky et al., 2012) and in turn, associated plant tissue (Bardgett \& Chan, 1999). Our data support such findings as the moss tissue and soil of Site 3 revealed the highest percent $\mathrm{N}$ of all sites (Table 4.3).

Further, recent studies imply that there may be an ancient "plantpollinator-like' mutualism between mosses and microarthropods (Rosenstiel et al., 2012). These two groups both show up in the fossil record in the late Ordovician-Silurian, 400-450 Mya, (Mishler \& Churchill, 1985) when terrestrial organisms were adapting to life on land, thus have evolved concurrently ever since. Collembola are the microarthropods that have been implicated in helping to facilitate moss sexual reproduction as they move about in the canopies (Cronberg et al., 2006, Rosenstiel et al., 2012), in essence relieving the "swimming" moss sperm of steep barriers to overcome. Highly correlated at Site $3(>0.75)$ is SOM, invertebrate abundance, Collembolan abundance, and moss genetic diversity (Fig 4.6), presenting the possibility that even in a geothermal system, microarthropods and their activities are intimately involved in the moss community structure and 
diversity. The overall invertebrate abundance and moss genetic diversity show strikingly similar patterns as we had hypothesized (Fig. 4.4 and 4.5), but the highest levels of both variables are not found in the coolest site as predicted, but in the median Site 3. We have seen little evidence of sexual reproduction of C. purpureus in LVNP (Eppley et al., 2011), yet we only have access to the park during half of the year, as it receives a large amount of snowfall during the fall and winter months, and it is therefore possible that we have missed phenological evidence supporting sexual reproduction in $C$. purpureus. Even if we overlooked often-cryptic sex expression, we have seen few dehisced sporophytes or old sporophyte stalks in these sites, as we would expect if sexual reproduction were regularly occurring, implying that perhaps sex occurs, but is rare. Sex-specific markers for C. purpureus have recently been developed (pers comms SF McDaniel) and using these across our populations will be an interesting way to assess the sexual structure of the populations to understand the underlying sex ratios and thus the reproductive potential of C. purpureus on the geothermal landscape.

With only one determined polymorphic microsatellite region in our sampled C. purpureus sites it is extremely difficult to draw conclusions about the overall genetic variation of our plants (Selkoe \& Toonen, 2006), but we can ascertain that our C. purpureus populations are not simply superadapted clones of the same genotype (SF McDaniel, pers comms). It is possible that the allele amplified is in a region of the C. purpureus genome 
experiencing rapid mutation and thus the variation we see is not a product of recombination. This explanation is worth considering with the low levels of sexual reproduction in LVNP (Eppley et al. 2011), but given the range and patchiness of $C$. purpureus among our sites, it is highly unlikely that the moss was distributed solely by fragmentation, and even less likely by clonal growth given the sometimes $>40 \mathrm{~m}$ distance between sites and low level of between population genetic variation in C. purpureus.

UV-B radiation have been cited as a cause of point mutations in Antarctic C. purpureus populations (Skotnicki et al., 2004) although others have argued against evidence of these findings (Clarke et al., 2008). Site 3 has minimal shadowing during the day and although we do not have UV radiation levels at our sites, we logged lux measurements and found that the light levels at Sites 3 and 5 are significantly higher than at the other sites and ultimately, Site 5, the hottest site is also the brightest site (Fig. 4.1a and d) also has the lowest level of genetic variation (SSWP) in C. purpureus gametophytes across the three meter diameter site. Therefore, if the region is rapidly mutating, we cannot determine that it is correlated to high temperature or light, nor that moss genetic variation, as far as we can tell is from mutation. There is also the possibility that interactions with invertebrates force fragmentation dispersal or that a physically induced somatic mutation causes genetic variation. Our data does however support 
the idea that there are complex, possibly overlapping drivers for diversity in an extreme environment.

By delving into the intricacies of this relatively un-explored geothermal, diminutive system and the respective roles for the biotic and abiotic players, we unearthed some interesting insights. Our thermal gradient showed that even in a geothermal system, where all temperatures are relatively extreme, temperature still plays a major role in structuring communities, but even the highest-temperature moss-based communities are not biologically limited or static. Further, we found evidence that moss genetic diversity is related to biologically active communities and to invertebrate, namely Collembolan, abundances. The moss-microarthropod mutualism has not to our knowledge, before this study, been tested in natural communities and we argue that the relationship deserves extended attention. Further tests could reveal empirical evidence of what may be the earliest plant-invertebrate transport mutualism. This study also confirms that a moss system is not simply a moss system to be viewed as scaffolding or as a major functional group that will all behave in a predictable manner, and further work investigating communities of more similar moss composition in extreme environments will help elucidate species-level drivers of community processes. This work demonstrates how moss-communities are part of a intricate, multi-trophic, interacting dynamic ecosystem that is as big and complex as any forest. 


\section{Acknowledgements}

We would like to thank the National Science Foundation, Doctoral

Dissertation Improvement Grant (no. 1210957, awarded to S. Eppley and E. Shortlidge) and the American Philosophical Society's Lewis and Clark Field Scholar Grant (awarded to E. Shortlidge) for the generous financial support in aid of this project. We would like to thank M. Balkan, S.C. Woll, J. Groody and C. Piedrahita for invaluable help in the laboratory and M. Balkan, M. Chmielewski and H. Prather for field support. Thank you to M. Magnusson at the National Forest Service for permitting and support. 


\section{Tables and Figures}

Table 4.1. Results of 2-way ANOVA between and among geothermal sites at two time points (PD and AM) and horizon (canopy, rhizoid and soil).

\begin{tabular}{lcccccc}
\hline $\begin{array}{l}\text { Source of } \\
\text { Variation }\end{array}$ & df & SS & MS & F & $\begin{array}{c}\text { \% Total } \\
\text { variation }\end{array}$ & $\begin{array}{c}\text { Significance } \\
\text { (P) }\end{array}$ \\
\hline \hline $\begin{array}{l}\text { Column factor } \\
\text { (time, horizon) }\end{array}$ & 5 & 2802 & 560.3 & 64.44 & 78.86 & $\mathbf{P}<\mathbf{0 . 0 0 0 1}$ \\
$\begin{array}{l}\text { Row factor } \\
\text { (site) }\end{array}$ & & & & & & \\
Residual & 20 & 173.9 & 8.696 & & & \\
\end{tabular}

Table 4.2. Results of 2-way ANOVA between and among invertebrate collection method results and study site.

\begin{tabular}{lcccccc}
\hline $\begin{array}{c}\text { Source of } \\
\text { Variation }\end{array}$ & df & SS & MS & F & $\begin{array}{c}\text { \% Total } \\
\text { variation }\end{array}$ & $\begin{array}{c}\text { Significance } \\
\text { (P) }\end{array}$ \\
\hline \hline $\begin{array}{l}\text { Column factor } \\
\text { (method) }\end{array}$ & 2 & 1445000 & 722641 & 3.011 & 25.92 & $\mathrm{~ns}, 0.11$ \\
Row factor (site) & 4 & 2210000 & 552511 & 2.302 & 39.64 & $\mathrm{~ns}, 0.15$ \\
Residual & 8 & 1920000 & 239996 & & &
\end{tabular}


Table 4.3 Values and ANOVA statistics for variables by site

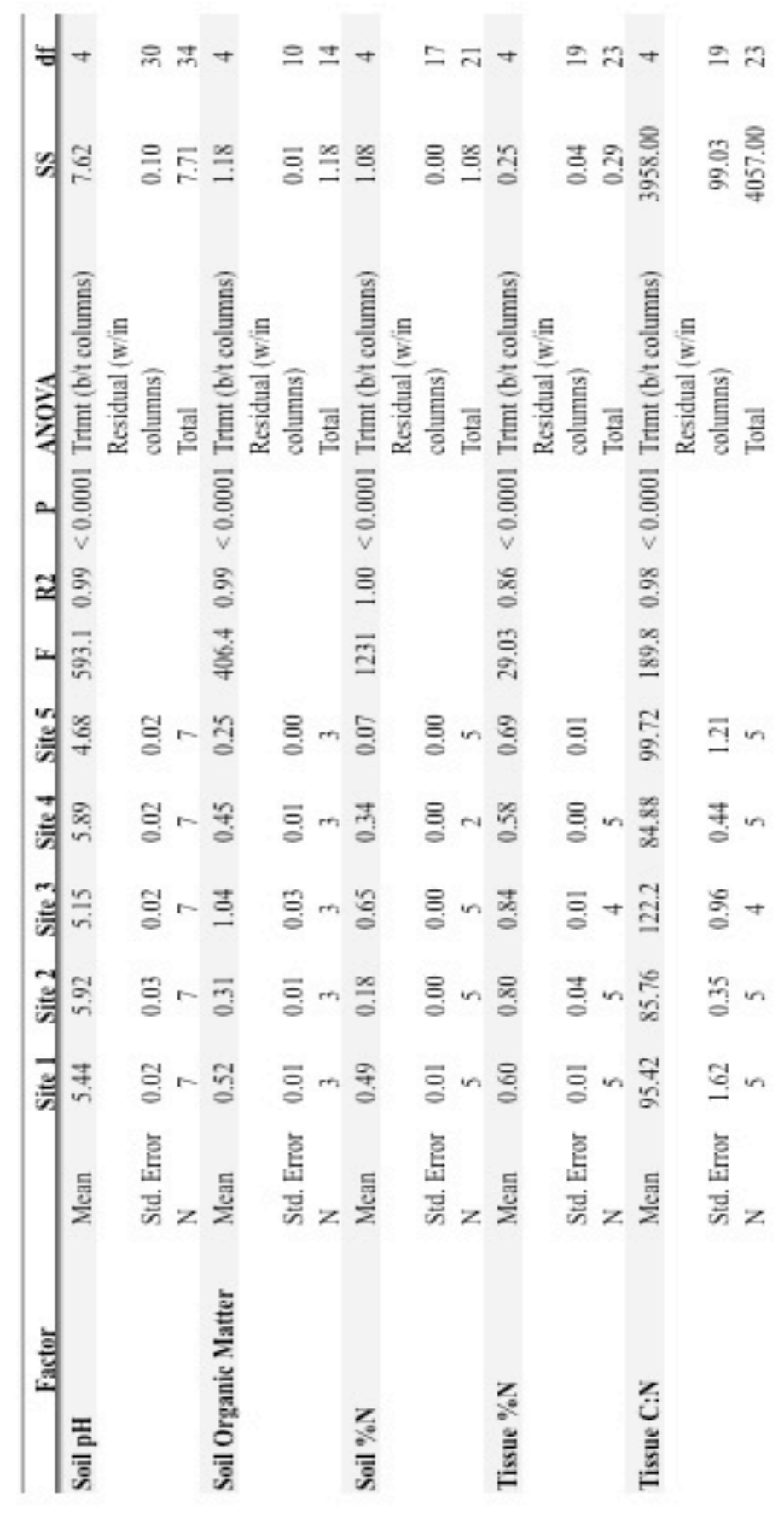


Table 4.4 Principal Component Scores from multivariate community analysis on correlation matrix.

\begin{tabular}{lrr}
\hline & $\begin{array}{r}\text { PC 1 } \\
\mathbf{( 5 1 . 4 \% )}\end{array}$ & $\begin{array}{r}\text { PC 2 } \\
\mathbf{( 3 7 . 3 \% )}\end{array}$ \\
\hline \hline Canopy Temperature & -0.37 & 0.146 \\
Collembola & 0.258 & 0.295 \\
Ergosterol & 0.385 & 0 \\
Invertebrate Abundance & 0.119 & 0.41 \\
Invertebrate Diversity (Gini-Simpson & 0.300 & -0.306 \\
Index) & 0.249 & 0.344 \\
Moss Variation (SSWP) & -0.385 & -0.056 \\
Oribatid & -0.200 & 0.394 \\
Relative Humidity (\%) & 0.344 & 0.121 \\
Soil Nitrogen (\%) & 0.300 & 0.279 \\
Soil Organic Matter & -0.263 & 0.308 \\
Soil Temperature & 0.119 & 0.41 \\
Lux & &
\end{tabular}


Figure 4.1 Abiotic profiles of five geothermal sites. Columns represent mean values, error bars are \pm SE. 1a) Temperatures of five geothermal study sites logged over eight days in the fall of 2012. b) Rhizoid-level temperatures recorded midday at each of five geothermal study sites in fall of 2012. c) Percent relative humidity of each geothermal study site recorded over eight days in October 2012. d) Lux levels of each site over eight days, October 2012.
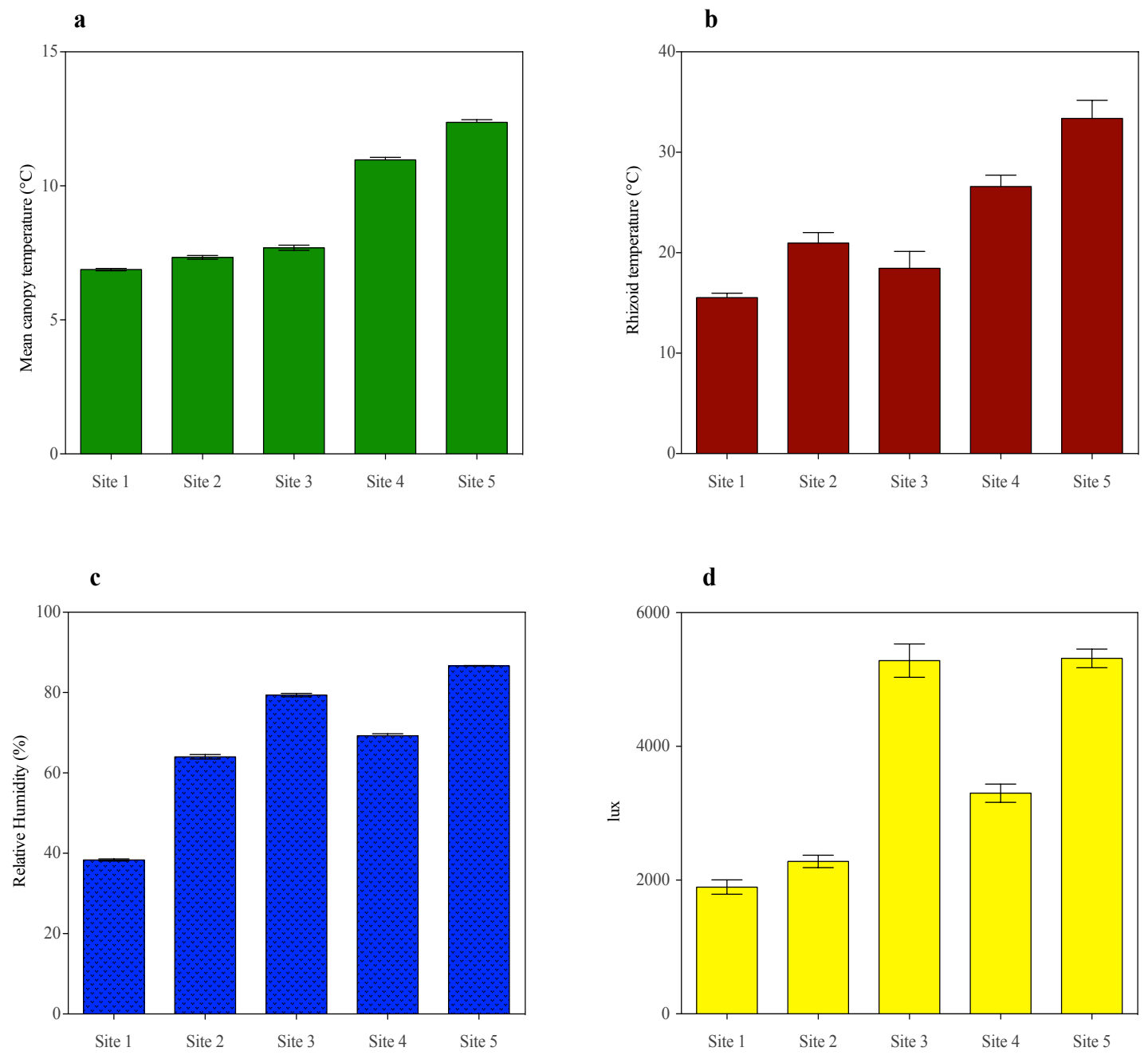
Figure 4.2 C. purpureus temperatures taken along a vertical gradient at two time points at five geothermal study sites, June 2013. Pre-dawn (PD) temperatures were measured just before sunrise, and AM and shortly after the sunrise. Each point is an average value of 4-6 measurements.

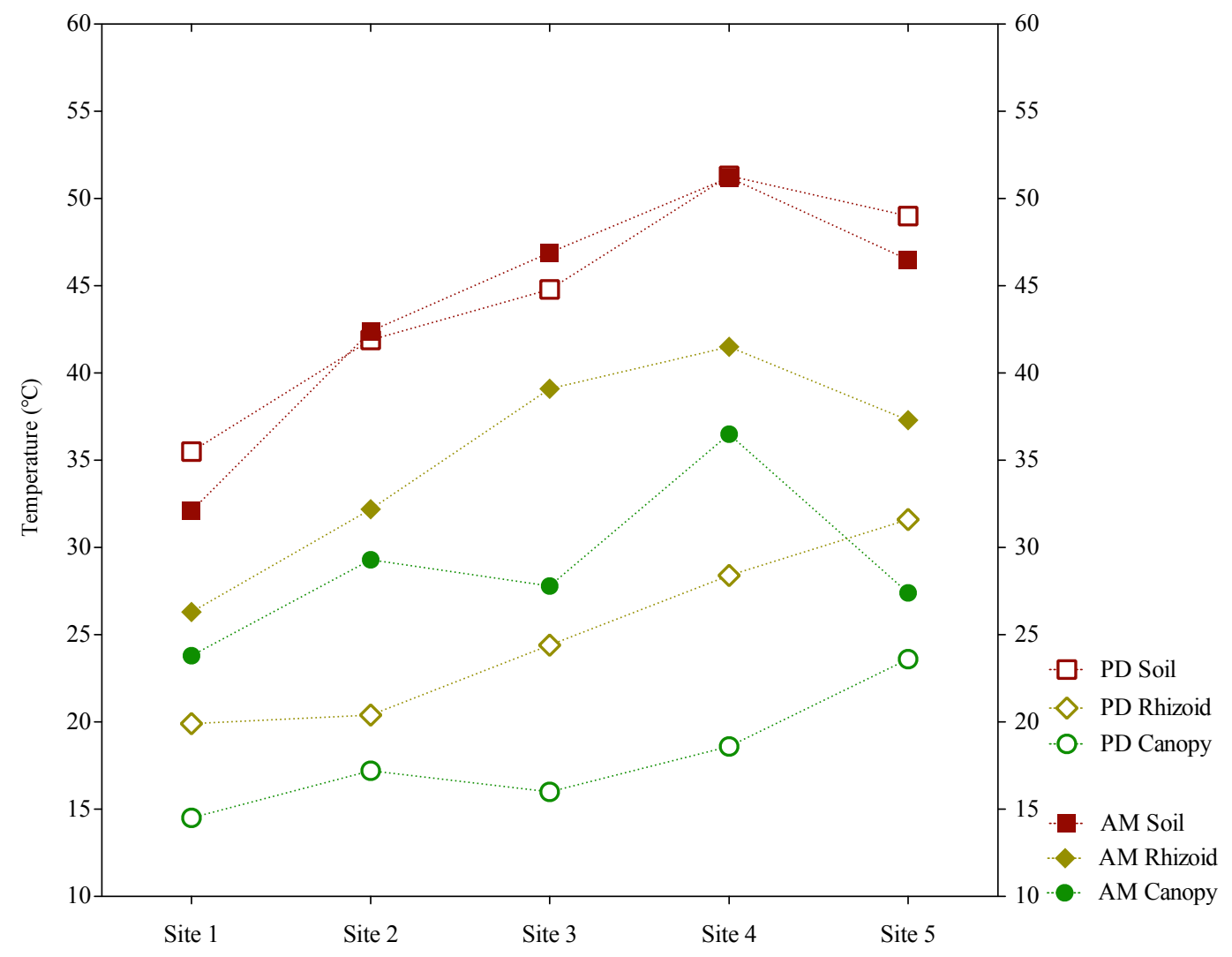


Figure 4.3 Invertebrate community group diversity and abundance. a) Gini-Simpson Index of invertebrate diversity of all counted individuals by site. b) Relative abundance of invertebrates counted by site and grouped by major taxa.

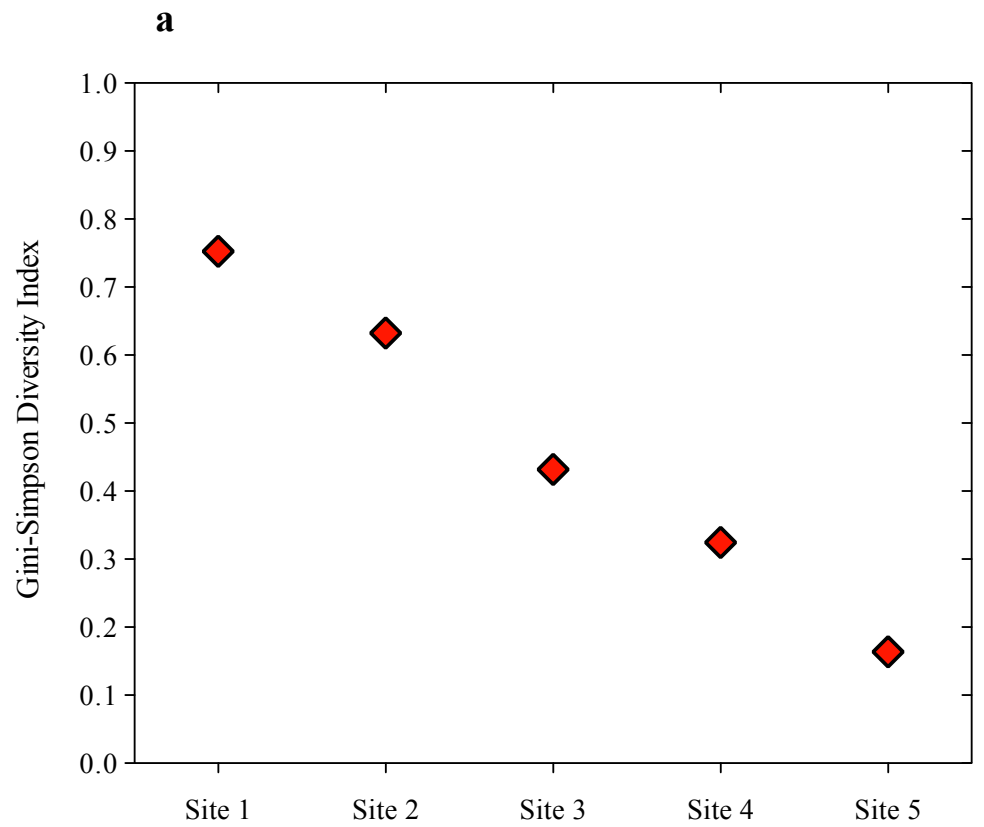

b

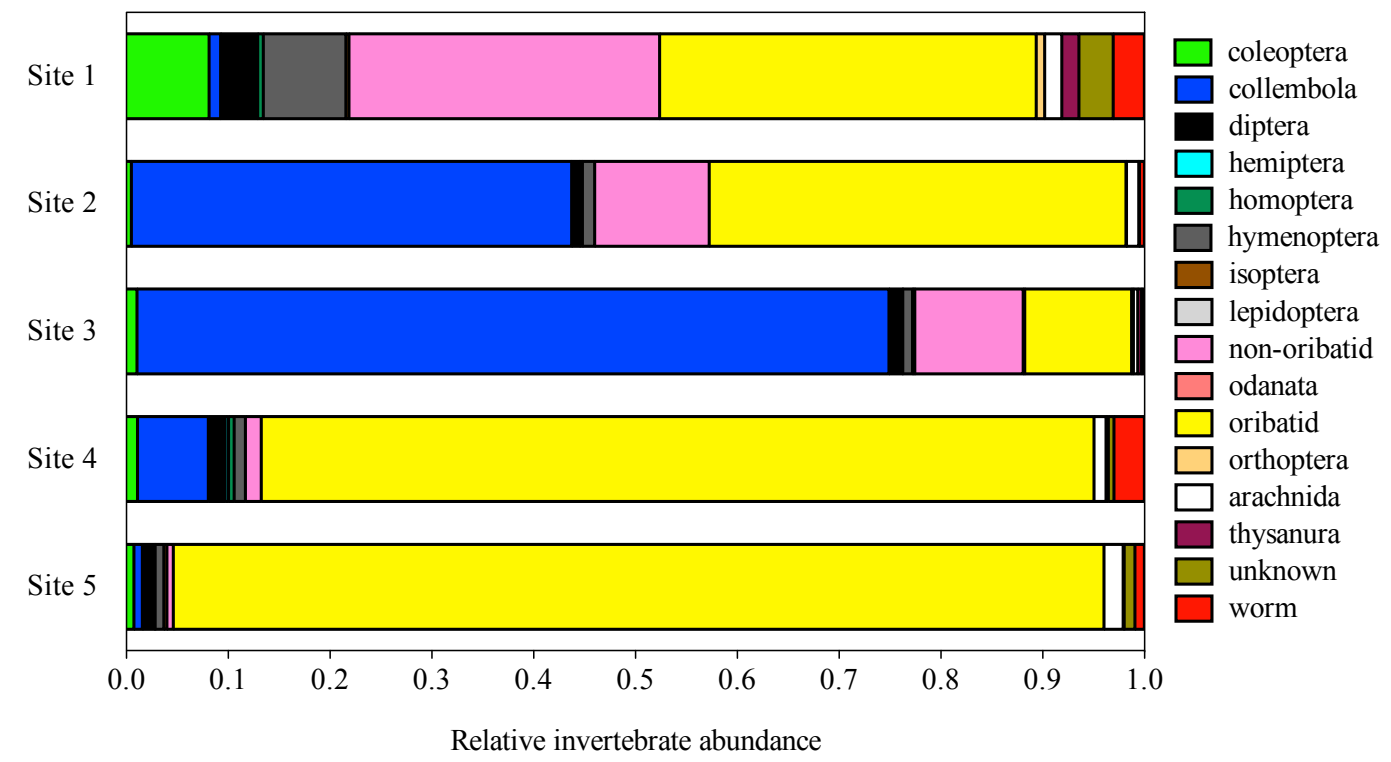


Figure 4.4 Total invertebrates collected by method at each of five sites. Total invertebrate numbers are symbolized a solid square, invertebrates extracted by funnels are represented by inverted triangles, and those collected from canopy-level pitfall traps are represented by open circles.

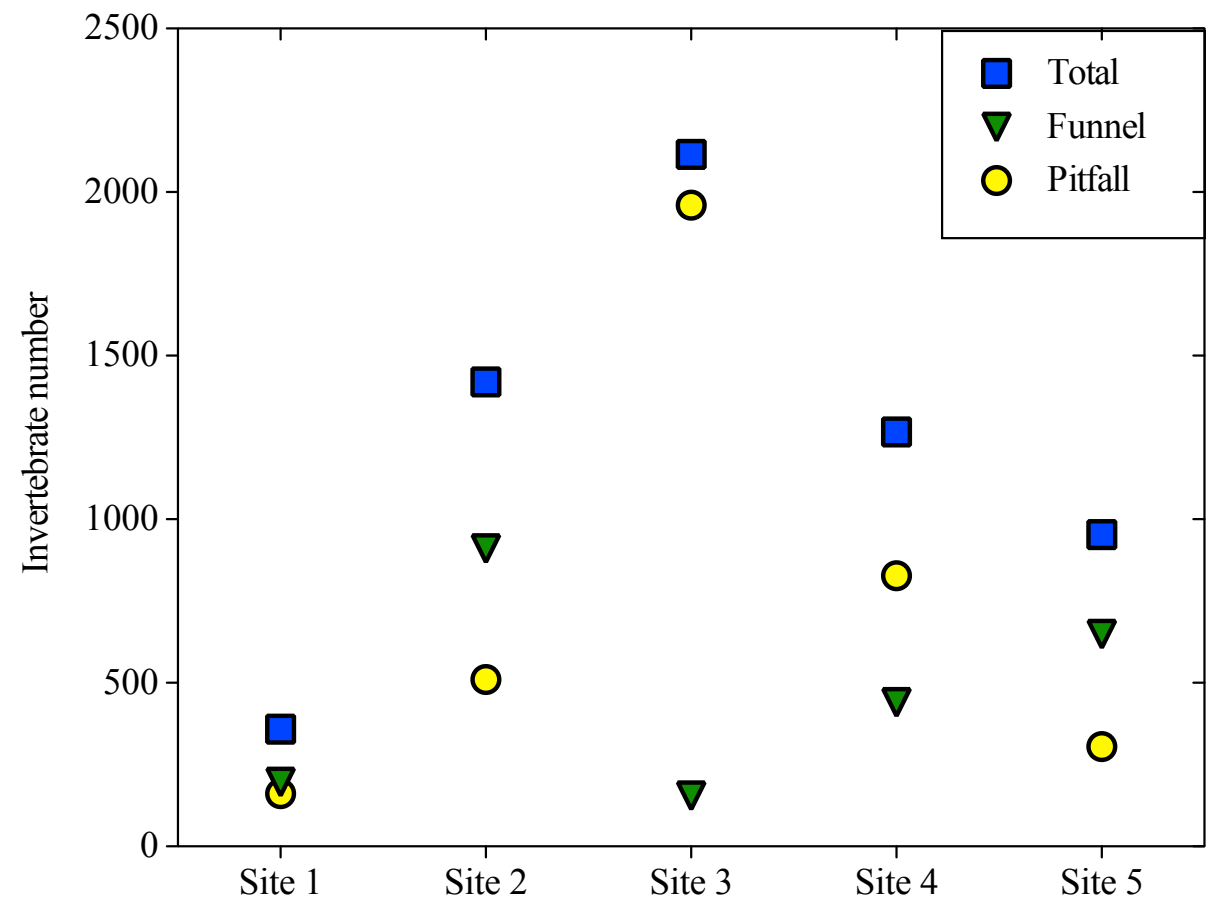


Figure 4.5 Genetic diversity of Ceratodon purpureus at one polymorphic microsatellite region. The Sum of Squares Within Population (SSWP) test statistics are derived from Analysis of Molecular Variance (AMOVA) test.

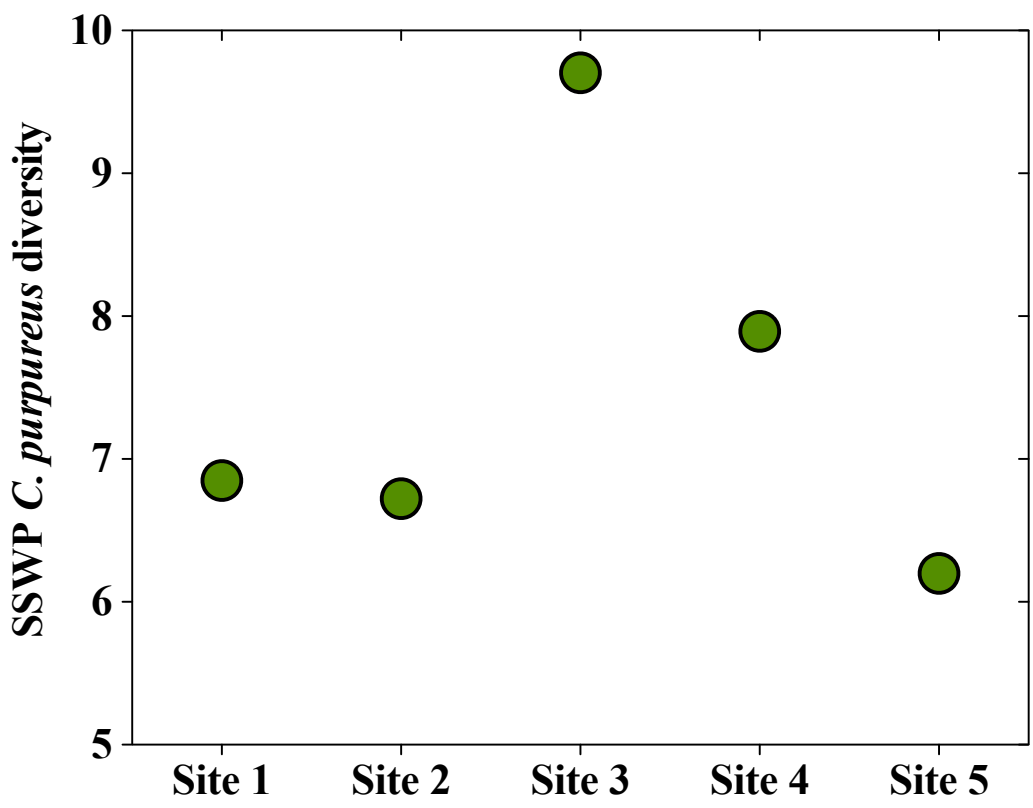


Figure 5.6 Principal component analysis of LVNP geothermal sites. PCA is a two dimensional ordination of correlations between measured biotic and abiotic variables at each of five sites. $51.4 \%$ of the variance in the data set is captured on the first component PC 1 , (x axis), and $37.3 \%$ is captured by the second ordination, PC 2 (y axis). Each vector direction correlates positively to the labeled variable. Each site in relation to the data that best describes it is represented numerically on the plot.

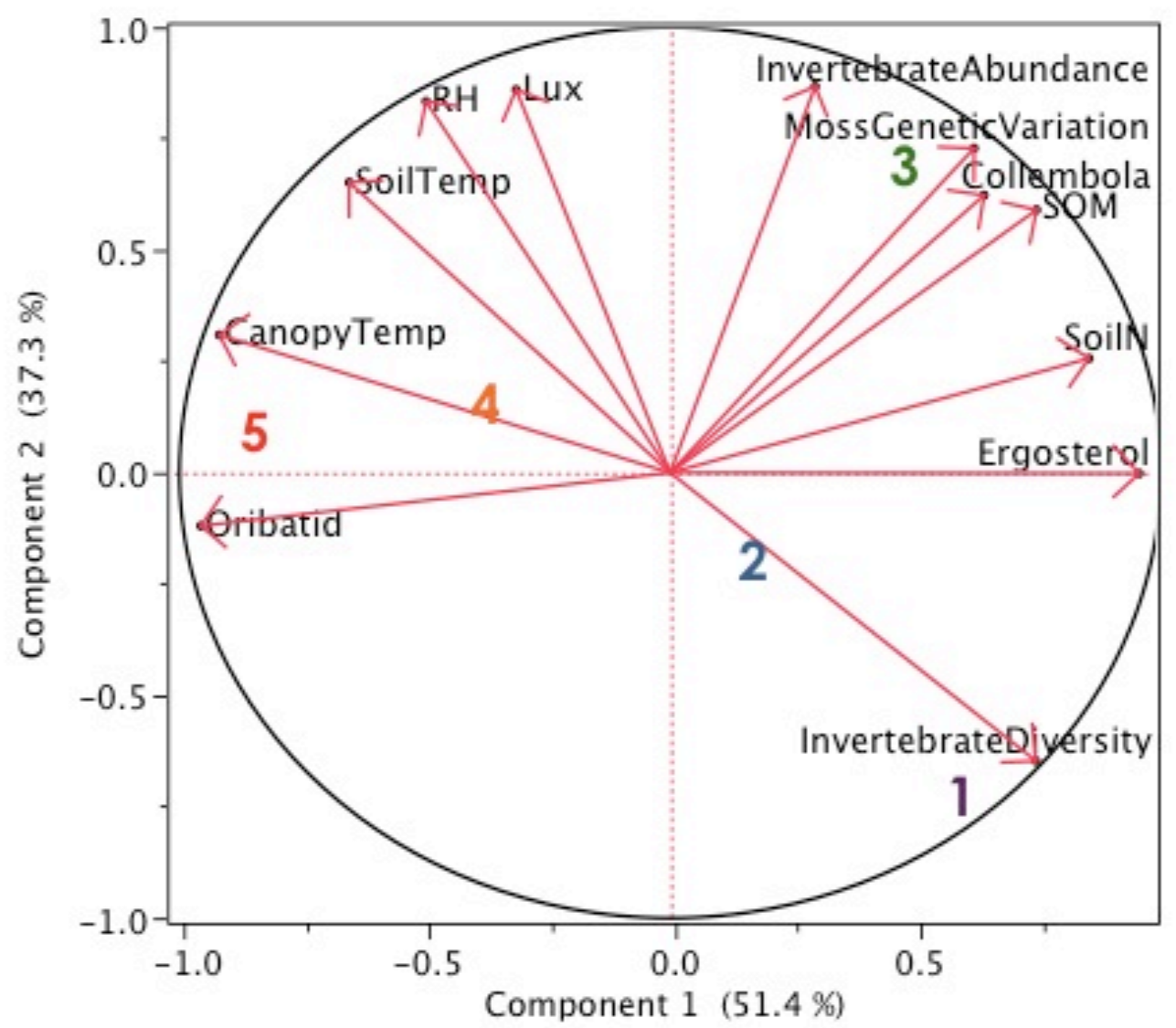




\title{
Chapter 5
}

\section{Warming reduces the physiological barrier to reproductive success in}

\section{Antarctic mosses}

\begin{abstract}
The western Antarctic Peninsula is one of the most rapidly warming regions on Earth, and the biotic communities inhabiting this dynamic region are reflecting these well-documented climatic shifts. Although often overlooked, mosses are the dominant vegetation in much of this ice-free region, and are important players in both local and global cycles, as well as in understanding the terrestrial ecology of Antarctica. In this study we investigated the effects of six years of simulated warming by Open Top Chambers (OTCs) on moss and lichen dominated communities on Fildes Peninsula, King George Island. We also focus specifically on a common dioecious moss species, Polytrichastrum alpinum. We surveyed vegetative cover in both warmed and un-warmed plots and assessed the relative investment of $P$. alpinum into growth, sexual reproduction, primary productivity, and stress defense. Our initial findings present significant differences between control and OTC plots. Our results show a general increase in moss and lichen percent cover under warming conditions as well as increased sporophyte production. We found distinct morphological and physiological shifts in P. alpinum patches under
\end{abstract}


simulated warming suggesting that the mosses are under less stress with warming conditions and are investing more towards primary productivity and sexual reproduction. Through in-depth studies of representative Antarctic mosses we can better understand species-level responses to warming and hopefully use that knowledge to predict functional group responses to warming. Taken together, our results imply that in mossdominated Antarctic regions, climate warming will change moss ecology, physiology and reproductive biology, thereby potentially impacting the entire terrestrial landscape through accelerated diversity and expansion on the Antarctic Peninsula.

Keywords: Antarctic Peninsula, ecophysiology, climate change, mosses, sexual reproduction, Antarctic flora 


\section{Introduction}

The western Antarctic Peninsula is experiencing rapid climate change. This phenomenon is attributed to increased intensity of circumpolar westerlies, warming in the tropical Pacific, and el Niño Southern Oscillation effects (Bracegirdle et al., 2008, Ding \& Steig, 2013, Etourneau, 2013). Recent data show that warming in the Antarctic is an increasing trend (Bromwich et al., 2014, Meredith \& King, 2005): a $2^{\circ} \mathrm{C}$ mean annual increase in atmospheric temperature since 1950 has been documented (Ducklow et al., 2007), an increased rate of Antarctic ice sheet and sea-ice melt (Liu et al., 2004, McMillan et al., 2014, Vaughan et al., 2003, Vaughan, 2006), and greater precipitation and snow accumulation (Van Lipzig et al., 2004).

Although Antarctica is rapidly warming, it still remains one of the harshest environments on Earth. It is extremely cold, windy, and dry, leaving relatively few organisms capable of thriving on its terrain (Kappen, 1993). Of the organisms hardy enough to survive, only two are vascular plants, yet there are 111 species of moss living in Antarctica, 109 of which are on the western peninsula alone (Ochyra et al., 2008). Therefore, mosses, the most diverse of the bryophytes, are dominant players on much of the increasingly exposed Antarctic landscape (Smith, 1994).

Mosses are non-vascular, diminutive, slow-growing plants, they are also successful, stress tolerant, complex photoautotrophs with a distinctive evolutionary trajectory, different from that of from their younger, vascular 
relatives (Shaw \& Goffinet, 2000). A key character allowing mosses to flourish trans-continentally is that they are poikilohydric, thus do not regulate their internal water status separate from that of their environment. Poikilohydry, combined with physiological adaptations that compensate for the effects of desiccation-rehydration and freeze-thaw cycles, allow mosses to suspend metabolic activity under water stress until a more suitable environment arises and recovery can begin (Bewley, 1973, Oliver \& Bewley, 1984a, Proctor et al., 2007). Thus, bryophytes can establish and persevere in harsh environments where many other organisms would perish. Retreating glaciers in Antarctica will reveal new potential habitats for colonization by these pioneering bryophytes and lichens (Convey \& Lewis Smith, 2006, Longton, 1988), where they are likely to progress into powerful ecosystem engineers, as they have demonstrated in the moss-dominated regions of the Arctic (Jassey et al., 2013) and the already ice-free regions of the Antarctic (Bramley-Alves et al., 2014).

Warming has been shown to speed up biogeochemical processes such as net mineralization, and increase plant growth and primary productivity (Aerts, 2006, Walker et al., 2006). Yet documented changes in Antarctic plant community responses to warming have primarily focused on vascular plants (Smith 1994, Day et al. 1999, Day et al. 2008, Hill et al. 2011, TorresMellado et al. 2011), with less emphasis on nonvascular cryptogam assemblages of bryophytes and lichens (Bramley-Alves et al., 2014, Robinson 
et al., 2003). When bryophytes are included in analyses they are often put into one functional group while vascular plant report findings are often species-specific (Klanderud, 2008). By studying species-level responses to warming, we can not only gain insight into specific organisms, but may also be better suited to extrapolate the results to understand how and why plant functional groups respond as they do to warming (Aerts, 2006, Chapin et al., 1996, Cornelissen, 1996). Plant functional group principles are not often applied to nonvascular plants, but in a moss-dominated landscape, understanding species-specific responses to warming could yield critical insight.

In situ simulations are often used to test the possible impacts of warming on susceptible communities and there is a history of studies using passive warming chambers (Open Top Chambers (OTCs)) in Antarctica (Bokhorst et al., 2007, Royles et al., 2013), but experiments that have included data on moss communities have been limited and with mixed results. For instance, warming experiments on moss-grass Antarctic communities suggest that at least for two moss species, warming decreases percent cover (Day et al., 1999, Day et al., 2008), presumably due to competition, although little change was seen in mosses in other mixed-plant communities (Bokhorst et al., 2007, Royles et al., 2013). Passive warming experiments, reviewed by Kennedy (1994) found that on substrate without plants, moss cover increased by $40 \%$ in two years, but in another Arctic studies, bryophyte and lichen 
cover decreased while vascular plant cover increased (Elmendorf et al., 2012, Wahren et al., 2005). Bryophyte responses to warming are likely to be different with and without associated vascular plants, thus making generalized predictions is challenging (Keuper et al., 2011).

None of these studies on Antarctic mosses have examined the effects of warming on sexual reproduction. Reproductive efforts have been surveyed in Antarctic mosses (Convey, 1994, Convey \& Smith, 1993, Smith \& Convey, 2002b), but to our knowledge, the potential impacts of imminent warming on moss reproductive biology have not. In fact, as far as we are aware, the effect of warming on moss sexual reproduction has not been addressed in any system, despite their dominance in the rapidly changing polar systems. Vascular plant communities in high arctic, alpine and Antarctic regions have exhibited increased sexual reproduction across plant taxa with gradual warming (Arft, 1999) and with experimental warming (Day et al., 1999, Klady et al., 2011, Molau et al., 1997). Plants increasing reproductive effort with warming presents clear implications for overall plant cover, genetic and biodiversity, as well for the functional ecology of the terrestrial landscape.

Sexual reproduction in mosses results in the production of genetically unique diploid sporophytes, containing thousands of potentially viable spores primed for release into the environment. Although mosses are extremely abundant, for many species, sporophytes are reported relatively infrequently in Antarctica, particularly in mosses with separate sexes (dioecious) (Ochyra 
et al., 2008, Smith \& Convey, 2002a). A hypothesis for sex limitation in mosses, particularly those maintaining dioicy, is that they are sperm dispersal limited, needing a constant water stream to carry the sperm across the landscape to the reproductively mature female moss. Yet, recent studies indicate mosses can have stress tolerant sperm (Rosenstiel \& Eppley, 2009, Shortlidge et al., 2012), that ubiquitous microarthropods in moss canopies can aid in sexual reproduction (Cronberg et al., 2006, Rosenstiel et al., 2012), and that dioicous mosses are more genetically diverse than would be expected with severe mate limitation (Van der Velde et al., 2001a, Wilson \& Provan, 2003, Wyatt et al., 1989). Such studies highlight the complexity of moss mating systems, but minimize the weight of dispersal limitation predictions.

Other explanations for infrequent or aborted sporophytes include:

resource limitation (Stark et al., 2000), growth trade-offs (Ehrlén et al., 2000), and investment in defenses against environmental stress that may in turn limit reproductive efforts (Convey \& Smith, 1993, Eppley et al., 2011, Stark et al., 2009). In general, plants under stress are often faced with "choosing" to invest resources into photosynthesis, growth and reproduction, or towards defense (Bazzaz et al., 1987, Herms \& Mattson, 1992). We predicted that mosses living with stress, particularly constant extreme environmental stressors such as those in Antarctica, may have limited investment in primary productivity and sexual reproduction compared to those under less environmental stress. 
Some the most extreme environmental limitations affecting Antarctic mosses will be relieved due to global warming, therefore we wanted to know how these changes may impact the moss-dominated vegetation of ice-free Antarctica by evaluating the community at large, and the physiological and sexual reproductive responses of one of the commonest and widely distributed dioicous Antarctic moss species, Polytrichastrum alpinum (Ochyra et al., 2008). We took advantage of already-established OTCs surrounding cryptogam communities in the western Antarctic islands. We present results of a much-needed assessment of the consequences of warming on Antarctic mosses by: 1) documenting differences between moss and lichen species assemblages in warmed and un-warmed plots and 2) performing focused studies on the stress physiology and reproductive biology of $P$. alpinum. Our data support the probability that the Antarctic Peninsula's moss-based plant communities will change in previously unprecedented ways as a result of ongoing climate change and that by better understanding the biology of moss functional groups we will be better suited to predict how the terrestrial landscape will change with warming.

\section{Materials and Methods}

\section{Study Site}

The study was carried out on Fildes Peninsula, King George Island $\left(62^{\circ} 00^{\prime} \mathrm{S}\right.$, $\left.58^{\circ} 15^{\prime} \mathrm{W}\right)$ in the South Shetland Archipelago, one of the largest ice-free areas 
in the Antarctic Peninsula (Ochyra 1998). The local flora covers large areas, both lichen and moss communities are well developed. In total, 61 moss species have been recorded on King George Island, of which 40 are present on Fildes Peninsula (Ochyra, 1998). The experiments were conducted on La Cruz Plateau ( $62^{\circ} 12^{\prime} \mathrm{S}, 58^{\circ} 57^{\prime} \mathrm{W}, 41 \mathrm{~m}$ a. s. 1.), located in the interior of Fildes Bay, which is oriented towards the Bransfield Strait, with moderate wind intensity and permafrost at $90 \mathrm{~cm}$ depth, influencing the water availability of the sites (Michel et al. 2008).

Nine Open Top Chambers (OTCs) and matched controls were established in 2008 on La Cruz Plateau by A. Casanova-Katny et al. (submitted). The entire site occupies approximately $100 \mathrm{~m}^{2}$ of a lichen and moss-dominated rocky plateau. The OTCs are of hexagonal outline, tapering with open top and assembled of $3 \mathrm{~mm}$ thick, transparent acrylic panels of 40 cm height, with a base area of $106.4 \mathrm{~cm}$ diameter; walls have small perforations $(1 \mathrm{~cm})$ and holes to allow for better air exchange and hence avoid excessive warming (Figure 1). These acrylic OTCs were tested for two years before being installed, and are similar in design to those previously tested for efficacy on Antarctica in Signy and Anchorage Islands (Bokhorst et al., 2007, Marion et al., 1997), and to those used successfully for other vegetative warming experiments (Bokhorst et al., 2011); for review of passive warming methods used in Antarctica). Moreover the OTCs resist the Antarctic climatic 
conditions. The matched controls were marked out regions of the same size within a meter of each OTC, but received no treatment.

\section{Methods}

\section{Abiotic conditions}

During the austral summer of 2014, temperatures inside and outside of two matched OTC and control plots were closely monitored with weatherproof data loggers measuring temperature and relative humidity (HOBO, Pro v2; Onset Computer Corporation, Bourne, MA) on 10 minute intervals over two weeks. The data loggers were established securely and camouflaged to avoid bird (Skua) disturbances at canopy level. Additionally, for one week, eight Ttype thermocouple wires logging temperature one time per second (s) (Hobo UX120; Onset Computer Corporation, Bourne, MA) were installed along a moss canopy gradient at 1) ambient-level (air just above the moss canopy), 2) canopy-level, in the photosynthetic moss canopy, approx. $0.5 \mathrm{~cm}$ below the air-canopy interface, 3) rhizoid-level (the root-like substrate attachment tissue of mosses just below canopy-level), and, 4) soil-level, $3 \mathrm{~cm}$ into the substrate, below the moss cushion, on both an OTC and a control plot. Weather stations have also been established at the site to monitor temperature, photosynthetically active radiation (PAR), and wind speed. The week in 2014 that we monitored conditions reported here was representative of normal Austral summer conditions on the Shetland Islands. 


\section{Moss and Lichen Community Analysis}

All plots were surveyed by sight for percent cover of the entire area within OTCs and control plots. Two researchers conducted the surveys and all points were agreed upon to eliminate single observer bias. Mosses were identified to genus and determined for percent cover and sporophyte presence or absence. Total percent lichen cover was included as an additional category.

\section{Physiology and reproductive biology of $P$. alpinum}

In order to adequately address complex concepts of stress and trade-offs, we chose to utilize one species of moss. We chose Polytrichastrum alpinum (Hedw.) G.L. Sm., a perennial, dioecious moss species that is common on Fildes Peninsula and produces sporophytes infrequently (Ochyra, 1998) and has hydroid cells. Mosses with hydroids can transfer water and minerals through specialized lignin-lacking conducting cells (Ligrone et al., 2012), potentially increasing the mosses capacity to actively obtain soil nutrients (Ayres et al., 2006). This trait makes $P$. alpinum a potential competitor in a warming Antarctica where increased temperatures increase available soil $\mathrm{N}$ (Aerts, 2006). Further, P. alpinum was the only dioecious moss species found in all of our control and OTC plots at La Cruz in our 2014 survey, yet does not occupy the majority of any one plot. Of the nine matched plots, three were randomly selected for sub-sampling. In each of three control and 
OTC plots, $P$. alpinum patches were marked along a $60 \mathrm{~cm}$ transect for in situ physiological assessment.

We determined $P$. alpinum moss canopy chlorophyll content by chlorophyll fluorescence (Gitelson et al., 1999) using a hand-held meter, (Opti-Sciences, CCM-300 Chlorophyll Content Meter, Hudson NH, USA), using standard manufacturer recommended protocols, three values were averaged to obtain each data point, and data were collected at three $P$. alpinum patches per each control and OTC along the $60 \mathrm{~cm}$ transect.

To non-invasively assess chlorophyll fluorescence parameters we measured maximum quantum yield of PSII (Fv/Fm). Fluorescence was measured on $P$. alpium patches that had been dark-adapted for 30 minutes prior to measurements (Bilger et al., 1995, Schreiber et al., 1995).

Chlorophyll fluorescence was measured with an OSp5 portable chlorophyll fluorometer (Opti-Sciences, Hudson, NH) using standard protocols, and fluorometer settings were chosen to optimize the fluorescence response during the various measurement efforts. Here we added one more control and OTC plot for measurements and collected five data points at each plot.

Six $1.9 \mathrm{~cm}$ diameter cores from each of three matched OTC and control plots were used for total pigments, protein, and antioxidant assays. Biochemical assays were conducted and results obtained by H. Kohler and G. Zúñiga at the University of Santiago, Department of Microbiology. Tissue was binned by treatment, cleaned of debris, and homogenized for immediate 
use or frozen for laboratory assays. To determine photosynthetic pigments (chlorophyll-a, chlorophyll-b and total carotenoids), $100 \mathrm{mg}$ of fresh plant tissue was homogenized in acetone and then diluted (10 times) in acetone and the solution was measured in the spectrophotometer. Absorbance was measured at 470, 649 and $665 \mathrm{~nm}$ (Wellburn and Lichtenthaler 1984).

For the extraction of proteins, fresh gametophytic material $(100 \mathrm{mg})$ was homogenized in a mortar with $1 \mathrm{ml}$ sodium phosphate buffer $50 \mathrm{mM}(\mathrm{pH}$ 7.2). The homogenate was centrifuged at $13,000 \mathrm{~g}$ for 25 minutes and the supernatant (crude extract) was used as a crude enzyme extract. The amount of protein in the crude extracts was determined by the Bradford protein assay (Bradford, 1976) with bovine serum albumin as standard.

To determine the amount of byproduct of lipid peroxidation (a product of oxidative damage) by the content of thiobarbituric acid reactive substances (TBARS), absorbance was measured at 532 and $600 \mathrm{~nm}$. The calculation was done using the molar absorptivity $(\varepsilon)$ of the adduct of malondialdehyde (MDA) with thiobarbituric acid (TBA) of $155 \mathrm{mM}^{-1} \mathrm{~cm}^{-1}$ using the difference between the wavelengths between 532 and $600 \mathrm{~nm}$. The results were expressed in terms of MDA equivalents (Ederli et al., 2004).

Environmental stress such as heat, light, cold and desiccation can result in elevated levels of damaging reactive oxygen species (ROS) in the plant cellular matrix (Møller, 2001). Organisms that are actively combating excess ROS often have elevated levels of protective antioxidant activity (Iba, 
2002, Martindale \& Holbrook, 2002). To assess the amount of oxidative cellular stress that $P$. alpinum plants are under, we tested the activity of ROS-scavenging enzymes (Gill \& Tuteja, 2010). Superoxide dismutase (SOD) activity was measured by recording the reduction of nitroblue tetrazolium to formazan by superoxide radicals (Beyer Jr \& Fridovich, 1987). The absorbance is recorded at $560 \mathrm{~nm}$, where a unit of enzyme activity was determined as the amount of enzyme needed to reduce the absorbance reading to $50 \%$ compared with a control containing no enzyme. Peroxidase (POD) activity was quantified as glutathione peroxidase (GPX) by modification of the method described by Curtis (Curtis, 1971). We measured the change in absorbance at $470 \mathrm{~nm}$ and activity was calculated using the molar extinction coefficient of $26.6 \mathrm{mM}^{-1} \mathrm{~cm}^{-1}$ at $470 \mathrm{~nm}$ for tetraguaiacol. Activity of ascorbate peroxidase (APX) is a rapid scavenger of cell damaging hydrogen peroxide molecules (Asada, 1997) and is often correlated to temperature stress (Iba, 2002). To determine APX activity we evaluated decreasing enzyme activity by measuring absorbance at $290 \mathrm{~nm}$ for $1 \mathrm{~min}$ of reaction from the extinction coefficient $(\varepsilon) 2.8 \mathrm{mM}^{-1} \mathrm{~cm}^{-1}$ (Chen \& Asada, 1989).

\section{Morphology and reproductive biology of $P$. alpinum}

Three additional $1.9 \mathrm{~cm}$ diameter cores were removed from each of the three randomly selected control and OTC transects. Cores were cleaned of 
substrate, and each shoot identified as $P$. apinum was counted under a stereo microscope (Olympus SZ61TR), scored as juvenile (very green with the leaves still appressed), mature (leafy gametophyte), or senescent (very little to no green tissue, blackening). Counting the number of shoots per core we can get an idea of moss canopy density. For each core, three mature gametophytes were randomly chosen for length measurement to the nearest $0.1 \mathrm{~mm}$. Each gametophyte was assessed for sex expression (as evidenced by presence or absence of male sex organs (antheridia), or female sex organs (archegonia)) under a stereomicroscope and/or compound microscope (Olympus CX31TR). If a moss gametophyte from another species was found in the core, it was discarded from the core although most cores were comprised solely of $P$. alpinum.

\section{Statistical Analyses}

To compare full community-level moss and lichen assemblages between OTC and Control treatments, percent cover data were ordinated with a two dimensional multivariate technique preferred for ecological applications (global Multidimensional Scaling (monoMDS); Bray Curtis distance on scaled data, 999 permutations; (Minchin, 1987) and full communities were assessed for statistical differences by treatment (OTC or control) with a post-hoc analysis of similarity (ANOSIM). Analyses were performed using the Vegan 
package (Oksanen et al., 2007) of the R Statistical software program (Team, 2013).

To test for differences among treatments, we used paired t-tests for analysis of total moss and lichen percent cover in matched plots. T-tests were performed to detect differences in the data mean across plots $\pm \mathrm{SE}$ for: temperature and relative humidity, differences between Polytrichastrum alpinum moss canopy chlorophyll content, dark-adapted Fv/Fm, antioxidant assays, total proteins and carotenoids, expression of sex ratio, shoot height and density, and age of shoots present. These analyses were performed using JMP10 (Inc, 2012).

\section{Results}

\section{Abiotic conditions.}

OTCs increased mean plot temperatures over control plots. Data loggers mounted just above moss canopies showed a significant temperature increase of an average of $2.3^{\circ} \mathrm{C}$ in the OTCs over control plots during two week interval of January 2014, (Fig. 5.1a; t=13.73; OTCs: $6.85 \pm 0.14 \mathrm{~N}=2228$, controls: $4.56 \pm 0.09, \mathrm{~N}=2228, \mathrm{P}<0.0001)$.

Continuous thermocouple temperature data also showed that OTCs significantly increase temperatures over the control in all four locations along the soil to air vertical horizon $(\mathrm{N}=65532$ and $\mathrm{P}<0.0001$ at all four levels) with the largest temperature increase with warming occurring at the canopy level. 
Ambient temperature means were $0.90^{\circ} \mathrm{C} \pm 0.01 \mathrm{SEM}$ and $2.39^{\circ} \mathrm{C} \pm 0.02 \mathrm{SEM}$ in the control and OTC plots respectively. Moss canopy temperatures were the most affected by the OTC treatment with a mean temperature of $0.80^{\circ} \mathrm{C} \pm$ $0.01 \mathrm{SE}$ in the control and $2.75^{\circ} \mathrm{C} \pm 0.01 \mathrm{SE}$ in the OTC plot. Rhizoid-level temperature was also lower in the control plot with an average of $1.0^{\circ} \mathrm{C} \pm$ $0.01 \mathrm{SE}$ in control, and $2.17^{\circ} \mathrm{C} \pm 0.01 \mathrm{SE}$ in the OTC. The soil temperature just below rhizoid level showed the least impact although still significant with control soil temperature mean at $1.87^{\circ} \mathrm{C} \pm 0.01 \mathrm{SE}$ and mean OTC soil temperature of $2.68^{\circ} \mathrm{C} \pm 0.01 \mathrm{SE}$ (Fig. 5.1b).

Alternately, relative humidity was significantly lower by an average of $12.2 \%$ in the OTC plots versus the controls, (Fig. 5.2; t=31.26; OTCs: $82.99 \pm$ 0.36SE N=2228; controls: $95.19 \pm 0.16 \mathrm{SE} \mathrm{N}=2228, \mathrm{P}<0.0001$ ).

\section{Moss and lichen community structure analyses.}

In total we found eleven recurring individual mosses, identified to species when possible, otherwise to genus including: Andreaea gainii Card. Bartramia patens Brid. (Bartramiaceae), Bryum sp., Ceratodon purpureus Hedw. Brid. (Ditrichaceae), Hennediella antarctica Hewd. (Pottiaceae), Pohlia nutans Hedw. Lindb. (Pohlnut), Polytrichum piliferum Hedw, (Polytrichiaceae), Polytrichastrum alpinum Hedw., G.L. Sm., (Polytrichiaceae), Sanionia uncinata Hedw. Loeske (Campyliaceae), and two unknown individual species, potentially Syntrichia sp. The lichen cover was 
dominated by Usnea aurantiaco-atra (Jacq.) Bory and Himantormia lugubris.(Hue) I.M. Lamb., which are mostly large fruticose and foliose lichens attached to the rocky substrate. There was a large percentage of rocky bareground in each plot, as well as $H$. lugubris covering the substrate. Results from the community structure analysis show that overall community composition (by percent cover) does not differ from control plots (Fig. 5.3, NMDS, Stress=0.13; ANOSIM test statistic $\mathrm{R}=-0.06, \mathrm{P}=0.76$ ). Similarly, alpha diversity (species richness) did not vary between treatments. The mean moss richness for the control plots was $4.7 \pm 0.67 \mathrm{SE}$, and OTC richness was a mean of 4.9 per plot $\pm 0.82 \mathrm{SE}$ ( $\mathrm{N}=9$ for each treatment). There were differences in abundance of each recorded individual species between OTCs and controls, all identified were slightly higher in the OTC plots than in the control plots except for two unidentified species (Unk 1 and Unk 2) found one time each in control plots (Fig. 5.4a). The overall percent moss cover was slightly higher in OTCs (Fig. 5.4a; OTC: $76.89 \pm 9.46$ SE; Control: $56.11 \pm$ 8.56 SE; $\mathrm{t}=2.23 ; \mathrm{N}=18 \mathrm{P}=0.06$ ) as was total lichen cover (Fig. 5.4b; OTC: 43.33 \pm 8.12 SE; Control: $29.44 \pm 6.04 \mathrm{SE} ; \mathrm{t}=3.05 ; \mathrm{N}=18 ; \mathrm{P}=0.02)$. Sporophytes were present in three of the nine OTC plots (77 total), and one of nine control plots (13 total). Overall sporophyte numbers were higher in OTC plots than controls, but due to the number of plots not expressing sporophytes at all, the difference was not statistically significant (Fig. 5.5; OTC: $8.56 \pm 6.21 \mathrm{SE}$, $\mathrm{N}=9$; Control: $1.44 \pm 1.44 \mathrm{SE}, \mathrm{t}=1.11 ; \mathrm{N}=9, \mathrm{P}=0.28$ ); the most sporophytes 
found in any one plot were 56 individual sporophytes counted in one OTC. All sporophytes identified were either B. patens (58\%) or H. antarctica (42\%).

\section{P. alpinum physiology and stress.}

Significant in situ physiological differences between $P$. alpinum patches growing in OTC and control plots were found in dark-adapted, canopy level $\mathrm{Fv} / \mathrm{Fm}$ values (Fig. 5.6a, $\mathrm{t}=2.62 ; \mathrm{N}=24 ; \mathrm{P}=0.01$ ). OTC mean $\mathrm{Fv} / \mathrm{Fm}$ values were $0.62 \pm 0.02 \mathrm{SE}$, and control were $0.55 \pm 0.02 \mathrm{SE}$. $(\mathrm{N}=24$ for each treatment). Similarly, chlorophyll content, reported as chlorophyll fluorescence ratio (CFR), of P. alpinum canopies was higher in OTCs, although not statistically significant. (Figure 5.6b; $\mathrm{t}=1.75, \mathrm{~N}=24, \mathrm{P}=0.09$; OTC: $0.82 \pm 0.03 \mathrm{SE}$; and controls: $0.75 \pm 0.03)$. Total protein content (mg g-1 fresh tissue weight) was significantly higher in the OTC tissue than the control tissue (Fig. 5.7a; t=21.05; OTC: $6.38 \pm 0.14 \mathrm{SE}, \mathrm{N}=3$; Control: $2.43 \pm$ $0.12 \mathrm{SE}, \mathrm{N}=3 ; \mathrm{P}<0.0001)$. Total carotenoids $\left(\mu \mathrm{g} \mathrm{mg}{ }^{-1}\right.$ protein) were greater in control plots over OTCs (Fig 5.7b; t=4.36; OTC: $2.46 \pm 0.50 \mathrm{SE}, \mathrm{N}=3$; Control: $5.33 \pm 0.43 \mathrm{SE}, \mathrm{N}=3 ; \mathrm{P}=0.01)$. A measure of internal oxidative damage by tissue lipid TBARS content of $P$. alpinum were significantly greater in controls than OTCs (Fig. 5.7c; t=8.75; OTC: $1.04 \pm 0.02 \mathrm{SE}, \mathrm{N}=3$; Control: $3.31 \pm 0.26 \mathrm{SE}, \mathrm{N}=3 ; \mathrm{P}=0.0009)$,

We found significantly increased antioxidant enzymatic activity in $\mathrm{P}$. alpinum tissue extracted from OTCs in all three antioxidant assays 
conducted (all activity is reported in $\mu \mathrm{mol} \mathrm{mg-1}$ protein). POD activity was greater in OTC tissue over control tissue (Fig. $5.7 \mathrm{~d}$; t=5.48; OTC: $0.13 \pm$ $0.002 \mathrm{SE}, \mathrm{N}=3$; Control: $0.53 \pm 0.07 \mathrm{SE}, \mathrm{N}=3 ; \mathrm{P}=0.0054$ ) as was SOD (Fig. 5.7d; t=7.27; OTC: $0.08 \pm 0.01 \mathrm{SE}, \mathrm{N}=3$; Control: $0.23 \pm 0.02 \mathrm{SE}, \mathrm{N}=3$; $\mathrm{P}=0.0019$ ), and APX activity (Fig. 5.7e; $\mathrm{t}=31.47$; OTC: $8.79 \pm 0.17 \mathrm{SE}, \mathrm{N}=3$; Control: $28.91 \pm 0.64 \mathrm{SE}, \mathrm{N}=3 ; \mathrm{P}<0.0001)$.

\section{P. alpinum morphology and reproductive biology.}

The mean number of gametophytes counted per P. alpinum core (a proxy for canopy density) was lower in OTC plots than control plots (Fig. 5.8; $\mathrm{t}=2.09$, $\mathrm{N}=18, \mathrm{P}=0.05$; OTCs: $23.0 \pm 3.03 \mathrm{SE}$; and Controls: $35.9 \pm 5.36 \mathrm{SE}$ ). Control plots contained more gametophytes per core in each ontological category counted (Fig. 5.8). Average height (mm) of mature gametophytes did not differ between treatments (mean of $3.0 \mathrm{~mm} \pm 0.20 \mathrm{SE}$ in controls, and $2.9 \pm$ 0.15 SE OTC gametophytes; $\mathrm{t}=0.30 ; \mathrm{P}=0.76 ; \mathrm{N}=54) . \quad$ P. alpinum gametophytes expressing male or female sex organs differed between plots, with more individual gametophytes actively expressing male and female sex organs in OTC plots over controls, but these results were localized in a few OTCs. In total, of the 18 cores examined, six contained gametophytes that expressed sex organs, four of which were from OTC plots, two from control plots (Table 5.1). P. alpinum sporophytes had previously developed in one of 
our OTCs where we did find sexually expressing individuals, but identified sporophytes were not from 2014 (capsule was completely dehisced).

\section{Discussion}

Recent data on the effects of climate change on Antarctica show that warming is rapidly occurring (King, 2004, McMillan et al., 2014, Vaughan et al., 2003), and this will inevitably change the terrestrial biology of the region. In this study we investigated the effects of warming on the moss and lichen cover that dominates much of the ice-free regions of Antarctica and found evidence that warming will indeed profoundly affect these important organisms by increasing overall biomass, relieving them of oxidative stress and increasing sexual reproductive efforts.

We found increases in total percent cover (Fig. 5.4) and sporophyte production in two common monoicous moss species (Fig 5.5) in moss-lichen dominated communities after six years of passive warming by OTCs. These data imply that in some Antarctic landscapes, abundances of lichen and moss cover will increase, as has been documented with warming of vascular plant communities in the Arctic (Klady et al., 2011, Robinson et al., 1998) and the Antarctic (Bokhorst et al., 2007, Day et al., 1999, Day et al., 2008). Decreased humidity is an inevitable product of warming and since mosses do not sequester water one would expect that decreased moisture would be detrimental to their growth. However, although the OTCs did significantly 
decrease relative humidity in our OTC plots compared to controls (Fig. 5.2), the total vegetative cover was still greater in OTCs. Warming studies in the Arctic have found that where moisture increases bryophyte cover decreases (Wahren et al., 2005), although the mechanism is unknown. In our study, percent cover of all species was marginally greater with warming compared

to the control, except for two "unknown" moss species, which were only found one time each in separate control plots (Fig. 5.4). Our species-level increases were insignificant yet consistent, and we propose that perhaps with a more refined scale of percent cover estimations, an even more profound effect could be revealed. Further, after only six years of warming we see a $20 \%$ increase in total moss cover in OTCs over the controls, indicating that perhaps the mosses are not always slow growing. We did not see any treatment effect on the number of species present (moss species richness was an average of five in both control and OTC plots), indicating no observable changes in biodiversity, but we plan to continue documenting these communities over time as the OTC and control plots are still active.

\section{Effects of warming on P. alpinum.}

We found reproductive and physiological differences between $P$. alpinum plants from warmed OTC environments versus those in control plots. $P$. alpinum that underwent experimental warming exhibited less physiological stress as evidenced by lower levels of antioxidant activity and greater PSII 
efficiency than in un-warmed P. alpinum (Figs. 5.6 and 5.7). Further, $P$. alpinum in warmed plots showed enhanced effort towards sexual reproduction (Table 5.1). These data indicate that perhaps climate change conditions will no longer be "extreme" enough to limit investment into sexual reproduction (Arft, 1999, Eppley et al., 2011) or to require high levels of defense compounds.

The gametophyte density of $P$. alpinum cores from the control plots was significantly greater than gametophtye density of warmed plots (Fig. 5.8). By growing in tight cushions, poikilohydric mosses trap water among gametophytes, making up a protective canopy presumably relieving the extent of water stress (Rice et al., 2008). The OTC plots are warmer and slightly drier, and although holes for air passage are incorporated into the OTC design, the mosses in OTCs still experience less wind, and we hypothesize that the distinct difference in canopy density is due to weakened pressure to form tight canopies (boundary layer) for protection from desiccation, cold, and wind. Typically, canopy density would correlate positively with primary productivity (Waite \& Sack, 2010), but we find evidence of higher PSII efficiency in the warmed mosses, indicated by greater Fv/Fm values (Fig. 5.6). Testing the effects of warming without changing the impact of natural wind could be difficult, but could uncouple the effects of increased temperature and decreased wind and relative humidity. 
Significantly higher levels of antioxidant enzyme activity were present in P. alpinum growing in our control plots versus those in the OTCs (Fig. 5.7). These data support that, $P$. alpinum, although a common stress tolerant Antarctic moss, is still investing heavily into stress defense. Researchers report mixed results (by species) of the effects of desiccation stress on moss physiology. For example, in experimental tests, mosses that experienced desiccation have been reported as having higher SOD activity and TBARS levels than mosses that did not experience desiccation (Seel et al., 1992a), indicating cellular stress control, and in other tests, some mosses carotenoid and chlorophyll content decreased with stress, but in other species, there was no pigment content change (Seel et al., 1992b). In general, if abiotic stress is decreased, antioxidant enzyme activity should also decrease (Apel \& Hirt, 2004, Sairam et al., 2002) as we found in our OTC plants after six years of elevated temperature. Our data show that warmed $P$. alpinum was investing more into producing reproductive structures (Fig. 5.5), total light harvesting machinery (Fig. 5.6), and exhibited less stress (Fig 5.7) than the mosses without simulated warming.

The total protein content of $P$. alpinum plants growing in the OTC plots was significantly greater than that of the control plots (Fig 5.7a), and chlorophyll content was nearly significantly higher (Fig. 5.6b) indicating that P. alpinum had access to greater nitrogen (N) pools in the OTCs. In vascular plants, high protein and chlorophyll content are typically correlated with 
increased N uptake (Bakken, 1995, Evans, 1989, Haag, 1974, Koranda et al., 2007), although less work has been done on the correlations in mosses.

Although most plant systems are $\mathrm{N}$ limited, mosses are efficient in acquiring $\mathrm{N}$, and they do so using different strategies than vascular plants (Eckstein \& Karlsson, 1999, Jauhiainen et al., 1998, Turetsky, 2003). Mosses take up nitrogen from the atmosphere through common symbioses with nitrogen fixing cyanobionts, these relationships span environmental and ecological gradients (Davey \& Marchant, 1983, DeLuca et al., 2002, Lindo \& Gonzalez, 2010, Pandey et al., 1992). In the substrate, mosses preferentially utilize inorganic $\mathrm{N}$ in the form of ammonia $\mathrm{NH}_{4}+$, but can also uptake organic $\mathrm{N}$ in the form of amino acids and peptides, further they can recycle $\mathrm{N}$ from their own senescent material (for review see (Turetsky, 2003). Using hydroids, $P$. alpinum is able to access $\mathrm{N}$ and other nutrients from the soil matrix particularly when water is freely available (Ayers et al., 2006), likely enhancing the rate of $\mathrm{N}$ scavenging by this species in the warmed plots. Additionally, $\mathrm{N}$-fixation rates of cyanobacteria are often temperature dependent (Housman et al., 2006) and yet are found to be locally variable (Kashyap et al., 1991, Robarts \& Zohary, 1987), including Antarctic cyanobacteria whose $\mathrm{N}$-fixation rates are highest in wet summer conditions (Davey \& Marchant, 1983). We speculate that the physiological state of $P$. alpinum is dependent on the interactions between the abiotic environment and the biotic interactions within these communities. Further, an increased 
pool of available nutrients would theoretically allow mosses the ability to partition more resources to sexual reproduction (Arft, 1999) as demonstrated by $P$. alpinum (Table 5.1) and in total sporophyte numbers from our warmed communities (Fig. 5.5).

P. alpinum is a successful Antarctic species yet has low documented sexual reproduction other than on geothermal soils of Deception Island (Smith, 2005) and along higher latitudes (Smith \& Convey, 2002b), likely due to environmental constraints (Convey \& Smith, 1993, Ochyra et al., 2008). Similarly, in our study, both male and female $P$. alpinum individuals that experienced warming showed increased effort towards producing reproductive structures, indicating that perhaps warming relieves environmental constraints on reproduction. If $P$. alpinum experiences decreased reproductive constraints due to increased temperatures and nutrient availability, there is new potential for diverse genetic individuals, possibly more fit to the changing environment to emerge via sexual reproduction. Although we cannot assess how other dioicous mosses will respond to increased temperatures in Antarctica, our data does imply that dramatic shifts, away from oxidative stress and towards primary productivity and reproduction are ahead for the common $P$. alpinum, and perhaps other mosses with similar life history traits. Identifying mosses that fall into a plant functional group with $P$. alpinum will be essential to understanding 
how far this study's findings may be applied as strategies and capacity for nutrient uptake and assimilation could differ among moss species.

We considered that perhaps mosses are indeed well adapted to the Antarctic landscape and thus perhaps we would find evidence of mosses being less healthy and experiencing more oxidative stress under simulated warming. Had we seen evidence of increased physiological stress with high antioxidant activity and low $\mathrm{Fv} / \mathrm{Fm}$ values in warmed $P$. alpinum plants, combined with increased reproductive effort, then a stress-induced sex hypothesis would have applied to our system (Bazzaz et al., 1987), but our data does not support this. Instead, this cohesive data set indicates that there may be a holistic shift in moss biology with climate warming.

Reports of how climate change has already impacted biotic communities of the Antarctic Peninsula reach broadly, including variations in annual penguin and phytoplankton populations, and the extended resulting trophic cascades due to increases in sea-ice melt (Croxall et al., 2002, Forcada et al., 2006, Fraser et al., 1992, Montes-Hugo et al., 2009). Simulated warming studies have revealed shifts in microbial community assemblages and increased rates of soil respiration (Rinnan et al., 2009, Royles et al., 2013) and microarthropod communities display species-specific responses to abiotic stress (Convey et al., 2002). There is even a cascading effect of penguin waste product on terrestrial nutrient dynamics (Park et al., 2007). All of these Antarctic organisms are connected to one another, often through 
the dominant moss vegetation from the penguin sourced $\mathrm{N}$ input to the invertebrates and grasses that live among mosses. Future studies on the combined effects of warming on species and community-level biogeochemical cycling and microbial activity in moss-dominated areas will be a next step to understanding how these data extrapolate to the functional ecology of the future Antarctic terrain (Jassey et al., 2013, Royles et al., 2013)

A meta-analysis of warming in the Arctic tundra revealed a general decline in species biodiversity, including the mosses (Walker et al., 2006), but to adequately predict trends in moss responses to climate change in the Antarctic, more empirical data on moss biodiversity under warming are necessary. Full plot analysis of our species data revealed no differences in overall species assemblages nor did any one particular species or group drive the modeled community profiles (Fig. 5.3), implying that the overall community change may be gradual and potentially difficult to perceive, thus we recommend further studies on individual species as well as moss-lichen interactions in order to obtain an accurate view of the effects of warming on the players in these communities. If warming does indeed promote overall cover, and induce increased effort towards reproduction as our data imply (Fig 5.4b, Fig. 5.5, Table 5.1), the range and diversity of mosses will increase as well as the potential range for each of the microbes, invertebrates, and vascular plants that all depend on the Antarctic mosses (Bolter et al., 1997, 
Casanova-Katny \& Cavieres, 2012, Convey, 1996, Convey et al., 2002, Ganzert et al., 2011).

\section{Conclusions}

This study is the first in examining effects of simulated warming on the physiology and reproductive biology of a common dioecious Antarctic moss species as well as its entire community. Our results indicate significant changes in the biology of our focal species, $P$. alpinum, a common Antarctic dioicous moss in response to warming as well as increased overall cryptogam biomass. Given the widespread occurrence of these organisms, is clear that any effort to understand the impacts of warming on terrestrial Antarctic communities should include the critically important species-level studies of nonvascular plant communities which will thereby allow us to better evaluate the greater functional impacts of these changes, from organism to ecosystem.

\section{Acknowledgements}

I would like to thank the Chilean Antarctic Institute (INACH) for generous financial and logistical support as well as A. Casanova-Katny for developing the OTCs and inviting collaboration, S. Eppley for financial support and the opportunity to conduct this research. Further I thank: A. Casanova-Katny, H. Kohler and P. Zúñiga for help in the field, and H. Kohler and G. Zúñiga for laboratory work. 


\section{Tables and Figures}

Table 5.1 Sex expression in P. alpinum cores of warmed and unwarmed plots. Six of 18 total cores assessed contained gametophytes with mature sex organs, four of which were from OTC plots and two were from control plots. This table shows the sex structure of the cores containing gametophytes expressing sex organs (number and percent) as well as the number percent of all total cores assessed.

\begin{tabular}{lccc}
\hline Treatment & $\begin{array}{c}\text { Gametophyte } \\
\text { number/core }\end{array}$ & Males exp. sex & $\begin{array}{c}\text { Females exp. } \\
\text { sex }\end{array}$ \\
\hline \hline Control & 42 & 0 & $1(2.4 \%)$ \\
Control & 25 & $8(32.0 \%)$ & $1(4.0 \%$ \\
\hline All Control Cores & $\mathbf{3 2 3}$ & $\mathbf{8 ( 2 . 5 \% )}$ & $\mathbf{2 ( 0 . 6 \% )}$ \\
\hline OTC & 10 & 0 & $1(10.0 \%)$ \\
OTC & 27 & $8(29.6 \%)$ & 0 \\
OTC & 26 & $5(19.2 \%)$ & 0 \\
OTC & 38 & 0 & $7(18.4 \%)$ \\
\hline All OTC Cores & $\mathbf{2 0 7}$ & $\mathbf{1 3 ( 6 . 3 \% )}$ & $\mathbf{8 ( 3 . 9 \% )}$ \\
\hline
\end{tabular}


Figure 5.1. Temperatures in control and OTC plots in January, 2014. a) Temperature $\left({ }^{\circ} \mathrm{C}\right)$ over two weeks at moss canopy level was significantly lower in control (green box) than in OTC plots (pink box). Boxes represent the data mean (at center cross) $\pm 95 \%$ CI; dots represent outliers. b) Temperatures (mean $\pm \mathrm{SE}$ ) of the vertical horizon in the representative OTC (green bars) and control plot (pink bars). Temperatures were logged at ambient, moss canopy, moss rhizoid, and $2 \mathrm{~cm}$ below rhizoid level continuously for three days, at each location temperatures were significantly higher in the OTC.
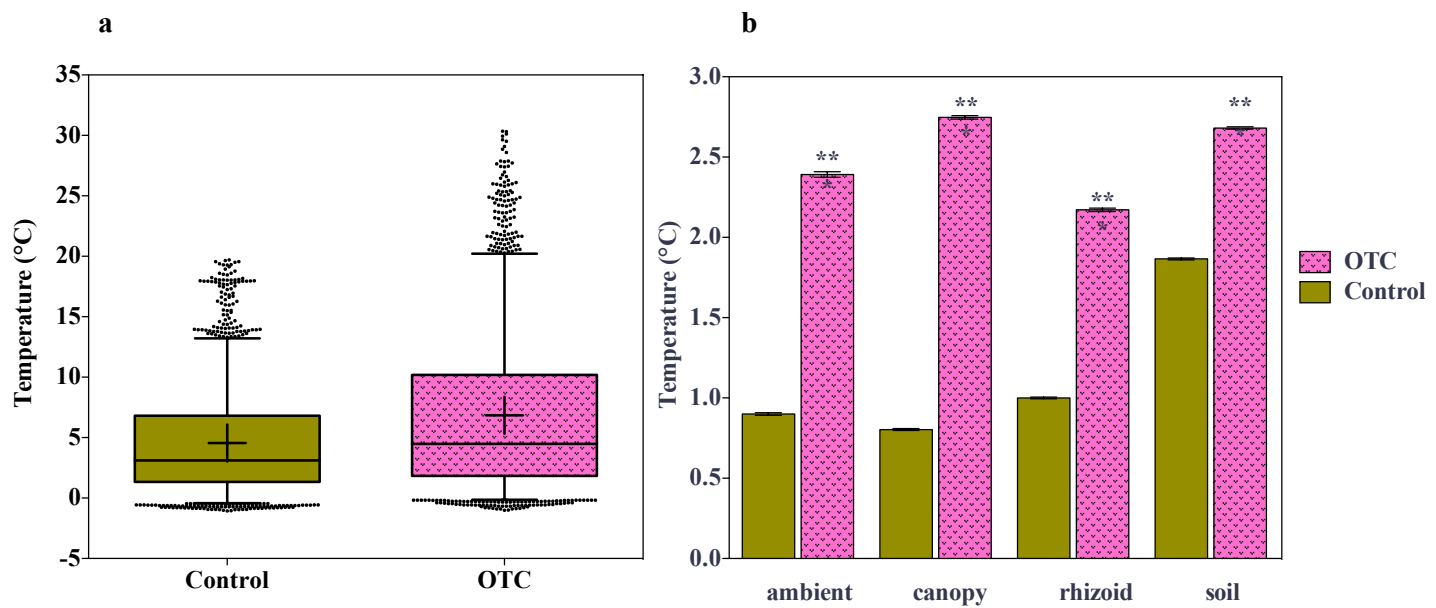
Figure 5.2 Relative humidity (\%) at moss canopy level. RH was significantly higher over two weeks in January 2014 in the control (green box) over the OTC plots (pink box). Boxes represent the data mean (at center cross) $\pm 95 \%$ CI, dots represent outliers.

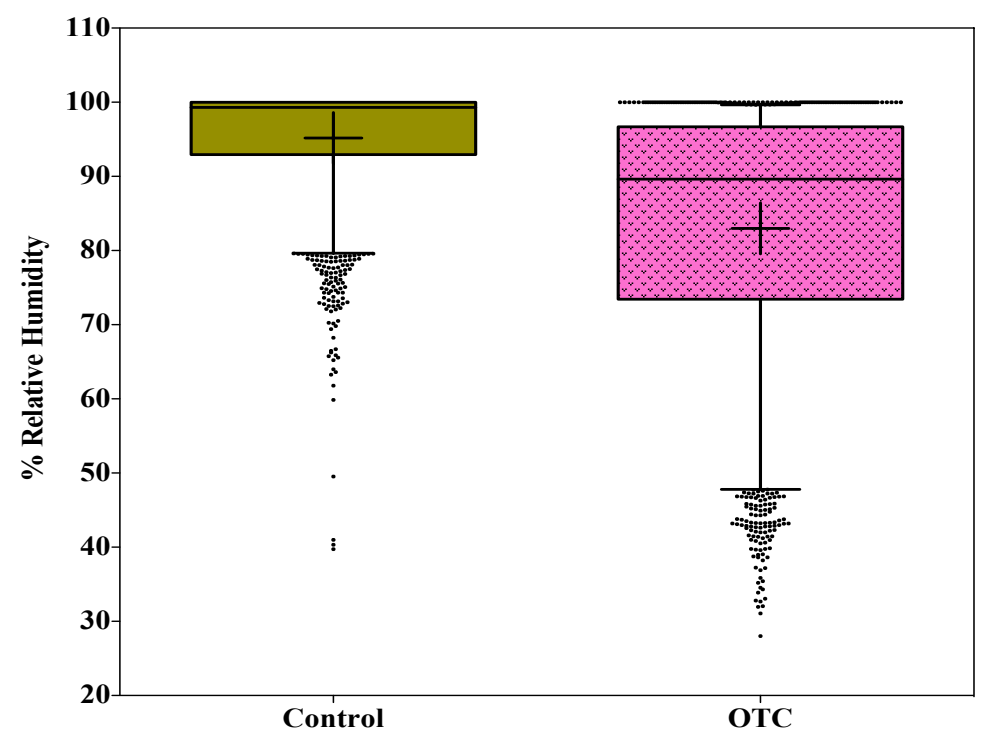


Figure 5.3 Community assemblages by plot. Two-dimensional nonmetric multidimensional scaling reveals no significant differences between the overall plant and lichen community composition by percent cover in plots with and without OTCs. OTC communities are represented by filled circles, and control plots are represented by open circles. Stress $=0.13$.

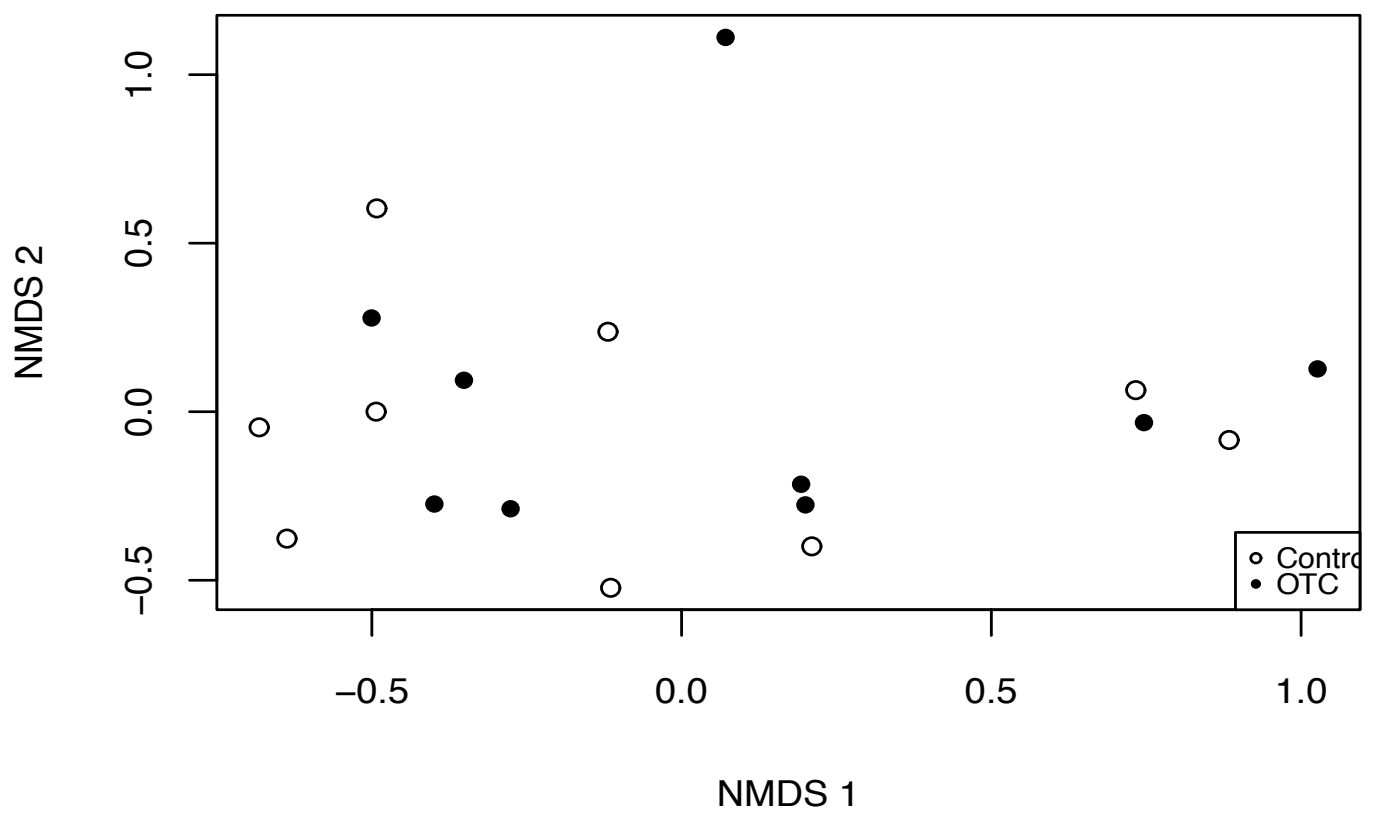


Figure 5.4 Species abundances by treatment. a) Comparison of percent cover (mean \pm SEM) of each recorded moss species from control (green bars) and OTC plots (pink bars). Individual species were not significantly different. b) Percent lichen cover is greater in OTCs $(\mathrm{t}=3.05 ; \mathrm{N}=18 ; \mathrm{P}=0.02)$, as is percent moss cover $(\mathrm{t}=2.23 ; \mathrm{N}=18 \mathrm{P}=0.06)$.

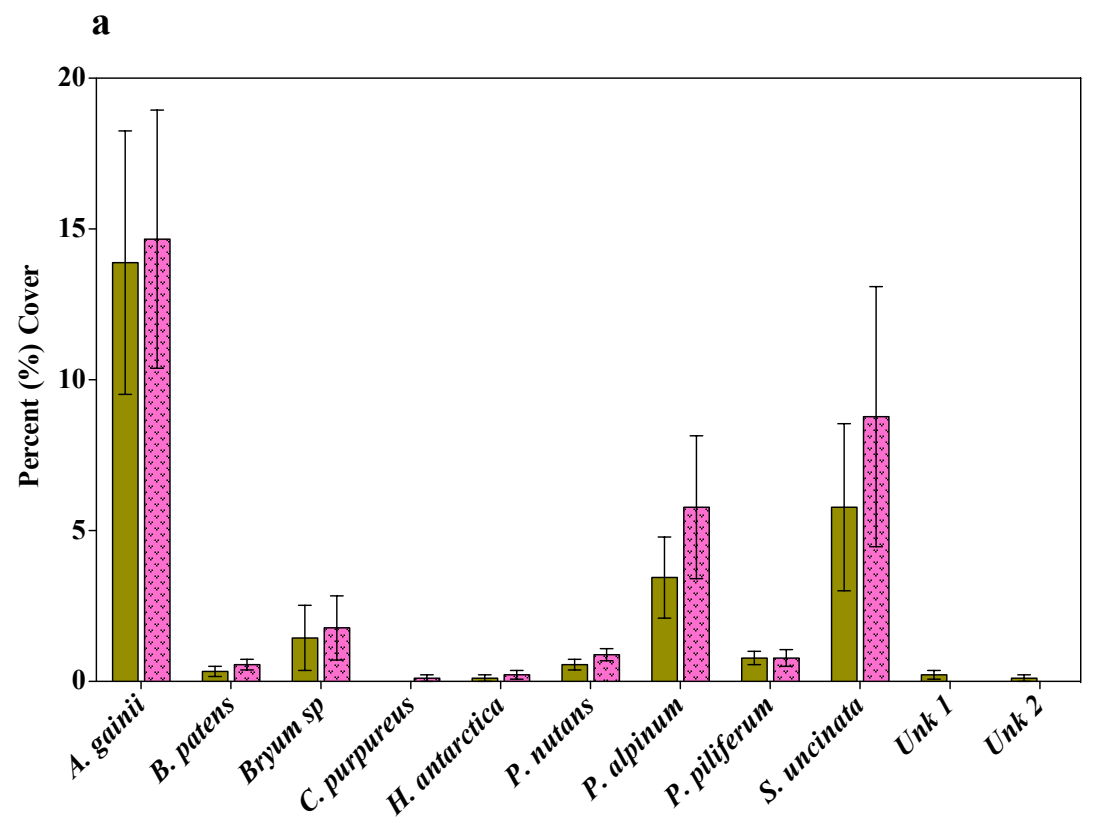

b

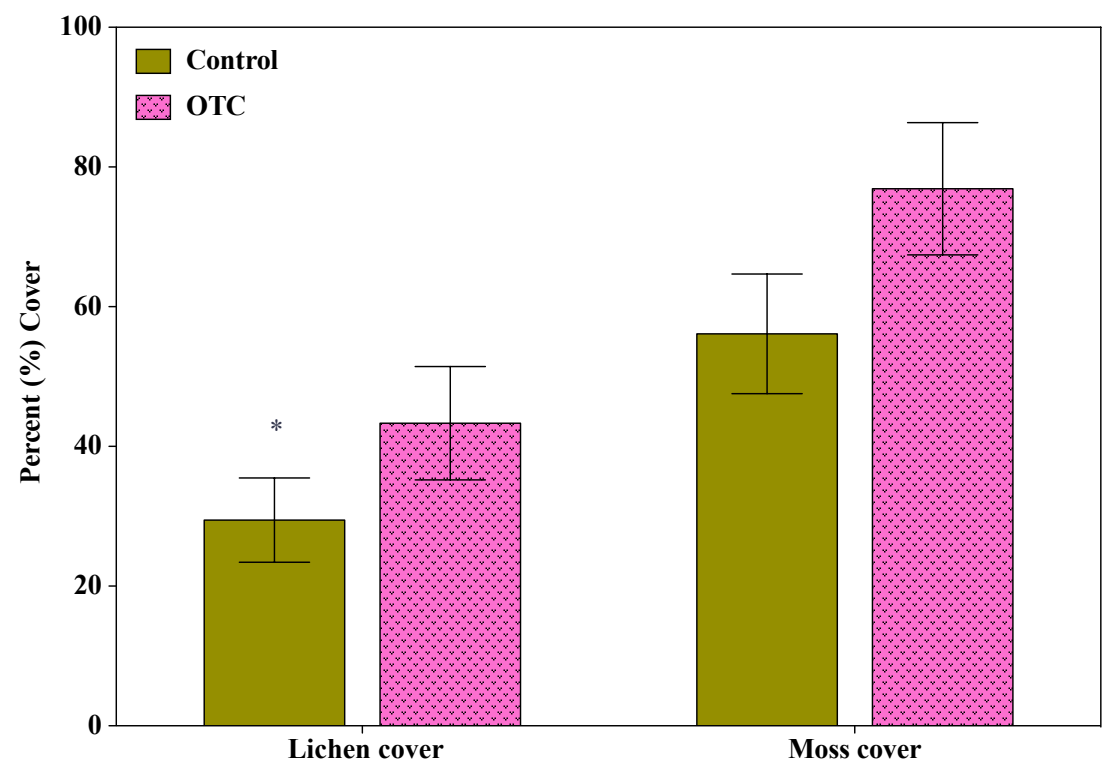


Figure 5.5 Sporophyte counts. Comparison of total sporophyte expression (mean $\pm \mathrm{SE}$ ) from control (green bar) and OTC plots (pink bar). There are more sporophytes expressed in the OTC plots than the controls, but results are not statistically significant $(\mathrm{t}=1.11 ; \mathrm{N}=9, \mathrm{P}=0.28)$.

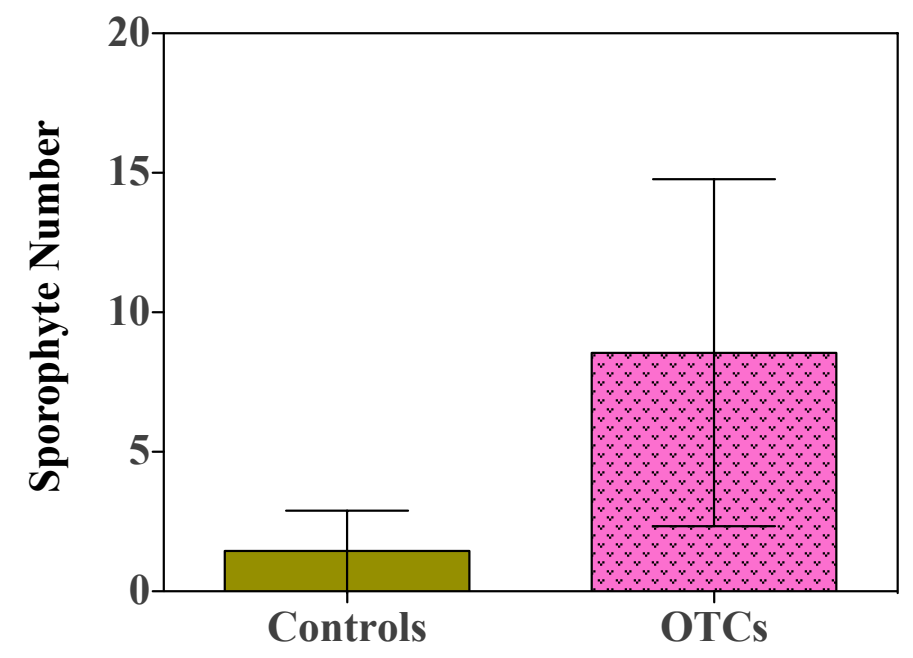


Figure 5.6 In situ physiological status of $P$. alpinum canopies in control (green bars) and OTC (pink bars) plots. Bars represent data mean \pm SE. a) $\mathrm{Fv} / \mathrm{Fm}$ values are significantly higher in OTC plots, indicating a more efficient photosystem ( $\mathrm{t}=2.62 ; \mathrm{N}=24 ; \mathrm{P}=0.01)$. b) Chlorophyll content of $P$. alpinum canopies was higher than in controls, but was not statistically significant ( $\mathrm{t}=1.75 ; \mathrm{N}=24 ; \mathrm{P}=0.09)$.
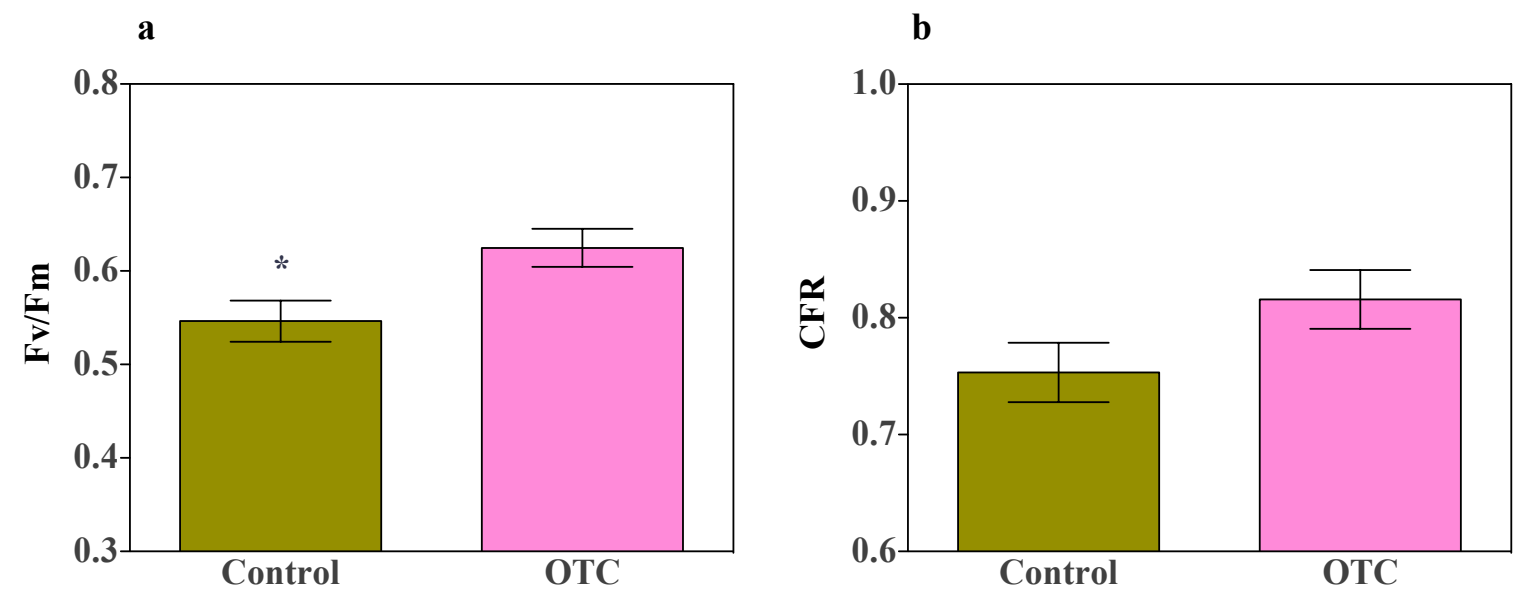
Figure 5.7 Physiological status of $P$. alpinum by treatment. P. alpinum cores extracted from control plots (green diamonds) and OTC plots (pink diamonds) differ significantly in all assays, $\mathrm{P} \leq 0.01$. $P$. alpinum tissue from each treatment was pooled and analyzed for laboratory assays. In all plots $\mathrm{N}=3$ for replicated assays, each value is indicated by a diamond and the horizontal bar represents data mean. a) total protein content of $P$. alpinum tissue, b) tissue carotenoids, c) TBARS, d) SOD activity, e) POD activity and f) APX activity. Each diamond represents a replicate assay of pooled $P$. alpinum tissue from either OTC or Control plots at La Cruz Plateau.
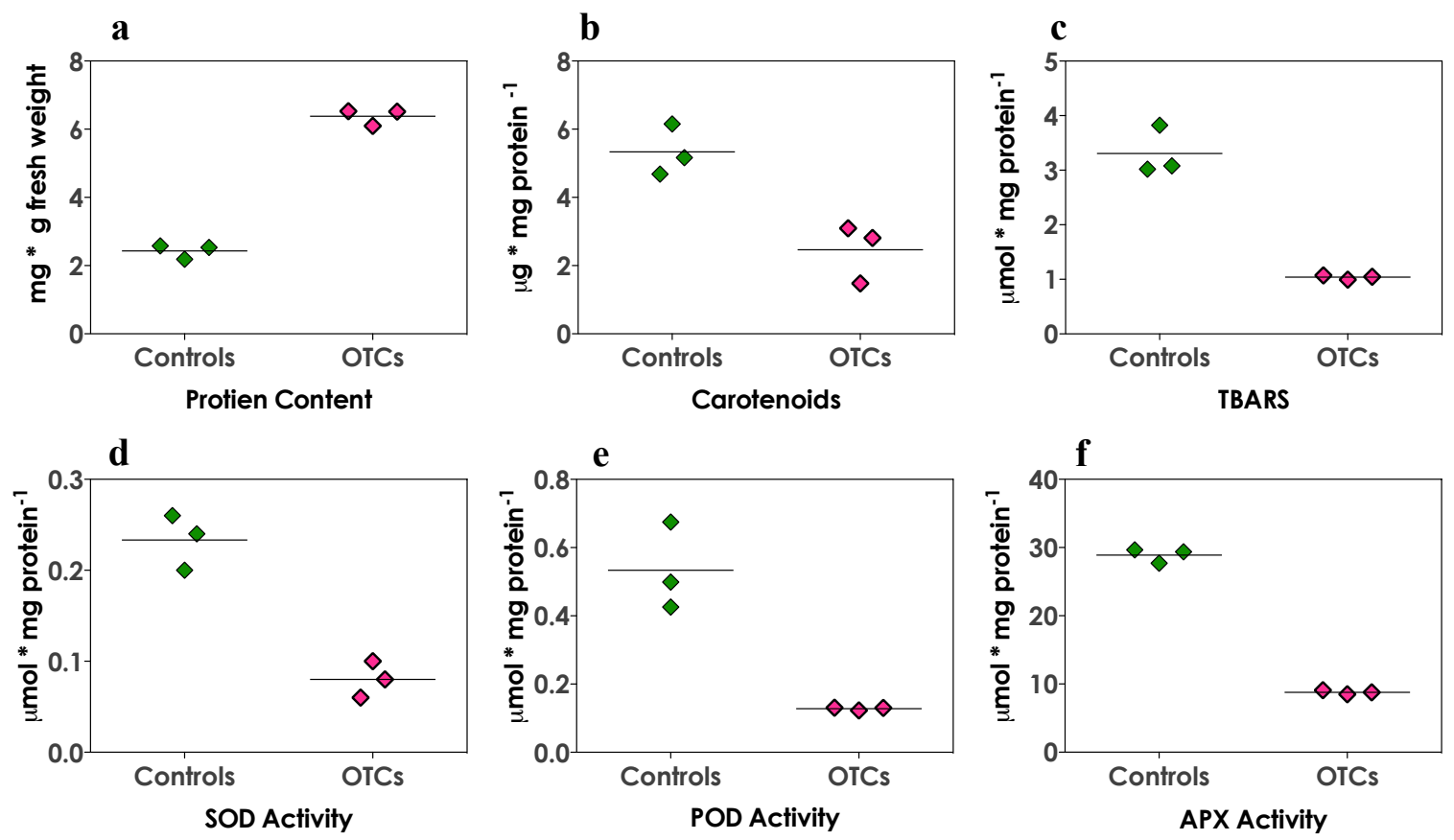
Figure 5.8 Composition of $P$. alpinum cores collected from control and OTC plots. Individual gametophytes of three ontological stages and total gametophyte counts (mean $\pm \mathrm{SEM}$ ) per core. There are significantly more total gametophytes per core from control (green bars) than OTC plots (pink bars) indicating a greater canopy density $(\mathrm{t}=2.09, \mathrm{~N}=18, \mathrm{P}=0.05)$.

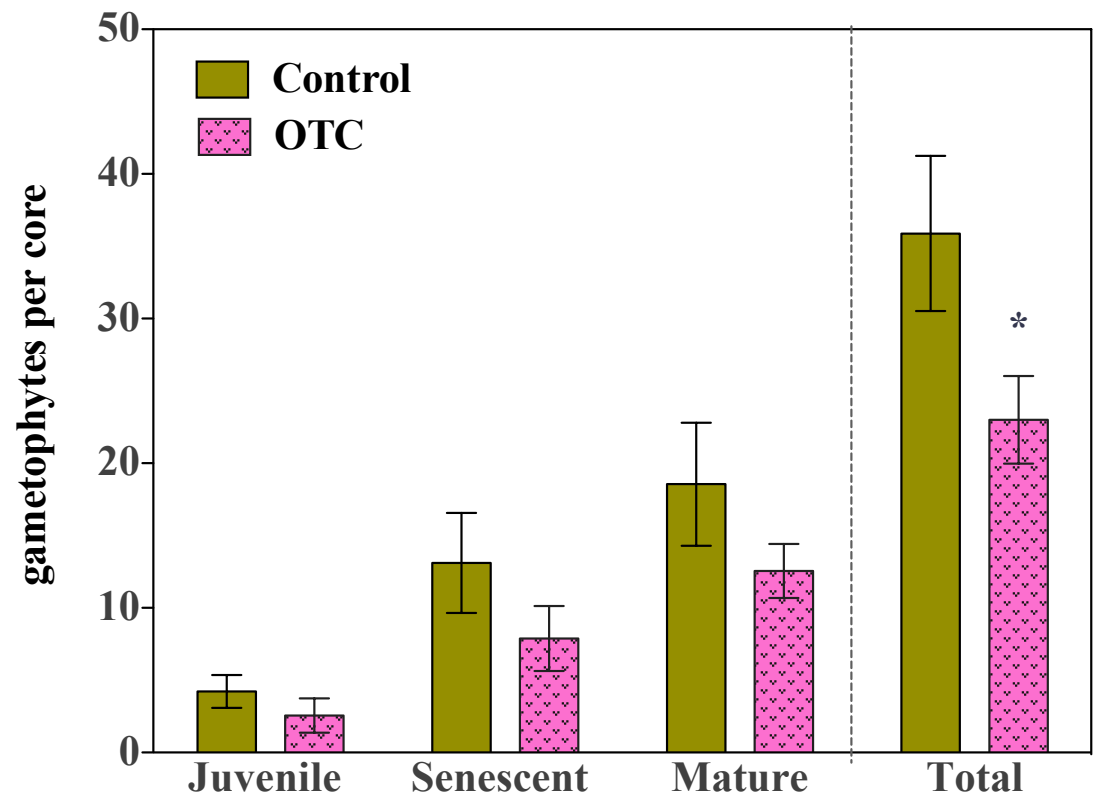




\title{
Chapter 6
}

\section{Mosscosms: a classroom activity to encourage ecosystem-level thinking.}

\begin{abstract}
Classroom science education is often discipline-specific with laboratories derived from exercises that "work", targeting an individual concept or fact, but not an entire system. Here, we discuss a project aimed at imparting complex, dynamic ecological themes to middle school students using a broadly adaptable, cross-disciplinary model. We developed a novel moss-based curriculum that is both ecologically relevant and facilitates opportunities for wide-ranging student experiences through intimate interactions with ecosystem subtleties. We describe how students can take ownership of their own, self-designed, hands-on, microcosm experiments and provide details on project execution and curricula. Representative examples of distinctive research-question-based projects, and a preliminary assessment of student thoughts and attitudes after participation are presented. We aim for biology educators and researchers to further replicate, modify, and formally assess Mosscosms as a modern tool for student comprehension of natural systems and the true scientific method.
\end{abstract}




\section{Introduction}

Ecosystems are complex dynamic entities that many research scientists spend their entire careers attempting to unravel and understand. To some extent, this same level of complexity can be found in populations, organisms, cells, and genomes. Thus, the type of thinking that is required by biologists of many types is thinking that considers interconnected systemic processes that are not necessarily limited to linear cause and effect. A life scientist's work investigates multi-dimensional networks that splinter and bifurcate, often only revealing truths about a particular question when attempting to answer an entirely different one. Uncovering and understanding these connections and relationships within, between, and among organisms in convoluted yet concise webs is fodder for scientists ranging from molecular biologists to climate system modelers. Further, the concept of connectedness has been identified as a key factor of effective science learning as outlined in Vision and Change's Systems objective: Living systems are interconnected and interacting (Brewer and Smith 2011). Multi-scale thinking is reflective of the way actual biologists conduct science, thus biology education should aim to imitate the craft.

Centuries of exploration, observation, prediction and experimentation took place before biological concepts emerged as comprehensive, testable scientific theories (Doebeli \& Hauert, 2005, Leibold et al., 2004, Levin, 1992, Loreau et al., 2001), and discussion continues among scientists concerning 
how to make sense of ecological complexity, and when applying a particular theory is defensible and appropriate (Ginzburg \& Jensen, 2004). For students learning biology and the scientific method, accepting and analyzing stochastic uncertainty should be a part of the process (DeBoer, 2000), as it promotes critical thinking skills and introduces students to "thinking like a scientist" (Duschl 2008). Researchers have found that it can be difficult for students to grasp concepts of defensible experimental design (Brownell et al. 2014) and ecosystem function both of which require thinking in a scientificsystems manner. Student difficulty in thinking scientifically may be a product of biology laboratory experiences often being confined to exercises where students all follow the same explicit steps through a manual, leading to a generic predicted outcome. The integrity of "cookbook" labs that "work" has come under increased scrutiny as research on the science of biology education grows (Bell et al., 2005, Brownell et al., 2012, Handelsman et al., 2004, Tanner, 2011). Prescribed learning may not encourage students to understand that hypotheses are not developed just to be "proven" correct. Alternately, active, research-based science activities in the classroom may allow students to think in individualized yet complex, whole systems-level ways, thus potentially achieving a more realistic and useful science education that mimics the genuine discovery-based scientific process (Hodson, 1996).

Through the National Science Foundation's (NSF) Cascades to Coast GK-12 program, we, a veteran teacher (JR Hashimoto) and a scientist (EE 
Shortlidge) developed a novel moss-based microcosm activity designed for middle school students and rooted in primary biological research.

Transcribing scientific research into "inquiry" activities that are appropriate, engaging, and scientifically relevant necessitates a blend of expertise in pedagogical and scientific realms, thus this project was ideal for a teacherscientist team with complementary skills (Keys \& Bryan, 2001, Peker \& Dolan, 2014). Our project model veers away from prescriptive learning and supports urgings for using live ecosystems in the science classroom (Eilam, 2012), as well as pervasive arguments for implementing inquiry-based, project-oriented learning (Anderson, 2002, Blumenfeld et al., 1991, Brownell et al., 2012, Thomas, 2000). Moss is a tractable, underutilized, complex, and globally important plant system that can facilitate multi-trophic, dynamic classroom activities. In this article we briefly describe Mosscosms in hopes to urge the use and modification of the curriculum, gain support for the development of an appropriate assessment of the project, and ultimately to encourage employing multi-trophic systems-level projects in the biology classroom.

\section{Background - Why moss?}

The 450 million-year-old moss system is an understudied yet growing model system for innovative primary research in the laboratory that can easily transfer into the classroom. Mosses are found in abundance on all seven 
continents (Glime, 2007), making them ubiquitously distributed, stress tolerant primary producers that sit prominently on the edges of life in a changing climate. Mosses are key players in global biogeochemical cycling and in engineering biotic communities (Jassey et al., 2013, Turetsky, 2003). Given their dominance at both poles, mosses will be instrumental in understanding early global impacts of terrestrial climate change (Lindo \& Gonzalez, 2010, Walther et al., 2002), a timely, relevant topic that is crucial to study in the modern biology classroom (Branch, 2013).

Further, mosses host a vast biodiversity of organisms including vascular plants (Andrew et al., 2003), (During and Tooren 1990), microbes (Davey \& Currah, 2006), and complex invertebrate and microarthropod communities (Andrew et al., 2003). Adding to previous work showing that microarthropods can transfer moss sperm (Cronberg et al., 2006), Shortlidge and colleagues discovered that springtails (common moss-dwelling microarthropods) play an integral role in moss reproduction, effectively fertilizing mosses while being guided by volatile cues emitted from the mosses (Rosenstiel et al., 2012). This phenomenon likens springtail activity in mosses to plant-pollinator interactions - another well-known, and important biological topic. Yet, unlike plant-pollinator systems, very little is known about intra-bryosphere interactions and what forces drive these relationships. 
In many regions of the world one can walk outside and grab a small clump of moss from off of a roof, the sidewalk, the trees, or deep in the forests - and each and every one of those clumps is a dynamic ecosystem that is bursting with life. In the classroom we employed mosses and springtails as a platform by which to study various organismal and ecosystem-wide interactions. Springtails have reliable life history traits, are used extensively in laboratories, and promoted for inquiry-based activities (Hopkin, 1997, Moore et al., 2000). Entire moss ecosystems are small enough to fit in a pocket, but are still relatively understudied. Mosses are easy to grow, maintain, and manipulate in the classroom for observations on ecosystem function and dynamics. They are ideal for experimental studies on: plant life cycles, growth, and development; symbioses and interactions; nutrient cycling; the effects of abiotic variables including environmental pollutants and precipitation, as well as simulated climate change conditions such as elevated carbon dioxide and temperature. We argue that a few patches of moss can uniquely provide an invaluable classroom tool for understanding organismal through ecosystem-level structure, function, and processes both in present-day and potential future conditions. There are numerous chances for students to uncover innovative information using the moss system, thus experiencing personally what lies at the heart of scientific discovery. 


\section{Mosscosms Project Summary}

Students developed and tested their own research questions about ecosystems by building microcosms of their own design on which they: observed, implemented regular treatments, made journal entries, collected and analyzed data, wrote formal lab reports, and ultimately designed and created a conference-style poster to be presented at the GK-12 Cascades to Coast regional annual STEM education conference (where one group won best overall project, 2013). The curriculum meets Oregon State Standards (6:2-8:2 and 6:3-8:3 Interaction and Change and Scientific Inquiry, respectively), and hits all four recommended objectives of the newly reformed National Academy of Sciences Framework for K-12 Science Education for Life Sciences (Quinn et al. 2012) (Supplement (S) 6.1).

Below we outline (I) our classroom logistics, (II) instructional background scaffolding, (III) Mosscosm-specific scaffolding, (IV) briefly describe a preliminary study that inspired Mosscosms and, (V) we describe the classroom experience from experimental design through the final project in Mosscosms (1-4), as well as a preliminary project assessment. Additional details on all of these aspects can be found in supplementary materials: (S6.2-6.9) and a mini-documentary (V6.1). 
I. Classroom logistics - We had six classes of 25-35 students each ( 170 students total) at an urban public middle school in Portland, Oregon. Each class met with Hashimoto four times per week (three days for 50 minutes (min), one day for $90 \mathrm{~min})$. Every class was comprised of mixed sixth-eighth grade students. Student Mosscosm groups of four were chosen by Hashimoto and included at least one student from each grade. The $90 \mathrm{~min}$ class periods were extremely useful for implementing Mosscosms, as $50 \mathrm{~min}$ classes are time-limited.

Shortlidge was in the classroom on average of one week per month throughout the school year, with the first four months focused on general scaffolding activities (see S6.2), allowing the students to become familiar with having a guest scientist in the classroom, and the teacher and scientist to develop a working relationship. Shortlidge spent more time in the classroom during initial development and finalization of the Mosscosms projects. Teacher and scientist planned out the week's activities in advance with Shortlidge taking the lead in the classroom on "GK-12 days". Mosscosms began in the second half of the school year (January) and culminated near the end in May. Hashimoto allotted a small amount class-time every week for students to maintain, observe and take notes on their Mosscosms projects, which were kept in the classroom.

II. Scaffolding - Generally, we followed an "examples first, bottom up" approach (Lawson 2009) to scaffolding activities, in effort to build a toolkit for 
students to access for problem-solving and tackling subjects seemingly advanced for their level (Hmelo-Silver et al. 2007). Scaffolding activities also allowed instructors to assess basal student knowledge. We used a combination of: projection slides, active classroom discussions, multimedia examples of organisms in their ecosystems, guided activities using field techniques and laboratory instruments, as well as data analysis and writing assignments (S6.2). Hand-outs helped keep students on task and attentive during lectures and discussions, and proved useful for students to source in later activities. Visual representation and corresponding written interpretations of data can be key to intuitive and concrete understanding of both quantitative and qualitative data (Cook, 2006), and we wanted the students to be able to analyze their Mosscosm data. Students practiced making data tables and graphing both on the whiteboard and on paper, followed by time in the computer lab using Microsoft Excel. Because we introduced students to inputting data and graphing early in the school year, at the end of the year when they had Mosscosms data, they already had skills to execute data input and graphing techniques. Students analyzed data as independent groups without halting project momentum to learn basic skills.

III. Mosscosm scaffolding - We provided "big picture" examples of ecosystems and held open discussions between and among students, researcher, and teacher about various aspects of moss ecosystems, immersing students into the practice of engaging with a scientific discourse and 
“community" (Applebee et al., 2003, Brickhouse, 1990). We then projected a written verbatim definition of the "bryosphere" from a peer-reviewed journal article (Lindo and Gonzalez 2010). Students thought about what the definition may actually mean, and were then asked rewrite the definition in their own words. This exercise was designed to help students see that they are capable of decoding complicated scientific information. Classroom discussion and debate followed where students talked through both the formal and derived definitions to reach a final consensus on what a bryosphere generally is: The interconnected biotic and abiotic factors that drive a dynamic moss-based ecosystem. This activity laid the foundation for students to start to build research-question based projects on their own bryospheres (S6.3).

IV. Preliminary study - In our first year (of two) as collaborators, our students developed hypothesis-driven projects that ended up serving as scaffolding for Mosscosms. Students were presented with the option of testing: springtail habitat quality, and/or food preferences with controlled variables. Most classes tested springtail food choice. After replicated experiments, student springtail data supported published rearing techniques (Hopkin, 1997). Data showing springtail population responses to food source in a controlled habitat is represented in Fig 6.1. These data were used in Mosscosms scaffolding, and the students appeared responsive to the fact that 
their peers' data was solid enough to be presented as a tool to inform their upcoming experiments.

\section{Mosscosms}

1. Experimental design. Groups were formed and students were told that they would get to create and experiment with their own bryospheres using the core materials of mosses and springtails, and it would be up to them to ask the questions. Students were tasked to come up with a group name, a clearly articulated research question, and an outline of their experimental design/methods to test the question. Groups pitched their project idea to the "panel" (teacher and scientist) where each proposal was reviewed, discussed, and then either accepted or recommended for revision (S6.4 - proposal details, S6.5 - sample research questions.) All proposals were eventually accepted after review.

2. Materials and Construction. Students developed a materials list for construction of the bryospheres. Some materials were to be provided (charcoal, pebbles, sand, moss carpets and springtails). The students were to bring all other items including habitats and other invertebrates (spiders, worms, centipedes etc.) if predators, competition, or biotic diversity was outlined in their proposal, thus encouraging project "ownership" from the beginning (Hanauer et al., 2012, Kennedy, 1994). Mosses are plentiful in the Pacific Northwest and easy to collect in a sustainable manner. If local 
collection is unreasonable for some geographic regions, mosses can be grown in the classroom as part of curriculum, or ordered them from a vendor (S6.6). Shortlidge rears springtails in the laboratory and they can be reared in the classroom from live caught populations, or obtained through an online vendor (S6.6). We discussed how to optimize habitat for all organisms. If a particular treatment could be deemed less than humane, we changed the project design.

Students decided who was responsible for procuring which items and had another conference session with the "panel" to assess: the completeness of the experimental design, potential issues with construction, and final materials lists (S6.6). Once each group was given the "ok" by the panel, the classes had one to two class periods to construct Mosscosms.

3. Onging Mosscosms. Throughout the year, every week, students were allotted in-class time to apply treatments and/or make observations on Mosscosms (S6.7 - expectations). Students made regular data entries in their lab notebooks (S6.8 - sample entry). Mosses grow slowly and springtails generally require about a month to reproduce, thus the Mosscosms were run and maintained in the background of class for three months. Monthly, we checked in with the groups to assess progress and to counsel on "failing" experiments. Counsel mostly consisted of discussion on why it is important to not change anything nor "fix" what may have gone "wrong", but to just go with the flow - these events challenged students and some experienced 
"disequilibrium", where students are allowed to struggle before getting to an understanding (Lawson 2009).

4. Experiment harvest and final data collection. By the end of five months, some Mosscosms were teeming with thousands of springtails, others were rotting, moldy, decomposing masses. In order to wrap-up, collect data, analyze, interpret, and prepare lab reports and poster presentations, we had the students "harvest" the mosscosms with about a month left in the school year (S6.9). In many cases, the research questions that students asked were predicated on how springtail populations would be effected by a particular biotic or abiotic factor. Each group outlined in writing what data they wanted to collect, why and how they would collect, and if it was appropriate to their project, before experiment harvests began. For many groups, simply counting the resulting springtail populations would be many days of work (springtails are $\sim 1 \mathrm{~mm}$ or less in length) and some treatments yielded thousand-fold increases in population. With guidance, students worked to develop a defensible subsampling method using grids and multiplication, which is representative of actual scientific data collection techniques.

For groups with less data from springtail population growth, or those collecting qualitative data, a variety of methods were used to gather data. Some students developed a color scale to evaluate moss color, others used equipment to measure variables such as water $\mathrm{pH}$, ambient $\mathrm{CO}_{2}$ levels, or 
chlorophyll content. Students collected data and complied it into tables and graphs. We led them through some analyses, and gave advice on variables to think about, but we mostly allowed the students freedom of interpretation and conclusion. Written lab reports and a poster representing their projects were required. The in-class poster-making process elicited excitement about "doing art in science class!" These experiences showed students how science can be a mix of various subjects and skill sets.

\section{Project Assessment}

Tests of Science Related Attitude (TOSRA) surveys were administered to students at the end of the school year. All students were involved in the project, thus negating the possibility of a "volunteer effect" challenging the equity of student response data (Kloser et al., 2013). The surveys were provided by the Cascades to Coast program and consisted of 30 questions designed to evaluate student attitudes toward science and the environment. Students could answer all questions on a scale from 1 to 5 (1=Strongly Disagree, 2=Disagree, 3=Not Sure, 4=Agree, 5=Strongly Agree). Questions 1-20 were aimed to assess student attitudes regarding whether they "enjoy" science and their interest in a "science career," with 10 of the questions being "positive" in connotation as it relates to a Strongly Agree response, and 10 with a "negative" connotation to Strongly Agree responses. Questions 21-30 were designed to assess students' attitudes towards "Stewardship." T-tests 
show that student responses indicate an overall significantly more positive attitude in response to questions 1-20 regarding whether they enjoy science, and have an interest in a career in science (Figure 6.2, $\mathrm{P}<0.003 ; \mathrm{N}=136$ ). See Table 6.1 for TOSRA Questions 1-30, and mean student response \pm SEM for each question.

In response to questions 21-30, the only "negative" question (q22: $I$ think you can get along fine without spending time in nature) received the lowest mean score (2.3 $\pm 0.09 \mathrm{SEM}, \mathrm{N}=134)$, and the question (q25: I think people can do helpful things for nature and the natural world) received the highest mean score (4.4 \pm 0.07 SEM, N=132).

As a supplement to the TOSRA assessment, we asked the students to write answers (all surveys/written answers were anonymous) to two questions on the back of their survey sheets: Q1) What was your favorite GK12 activity and why? and, Q2) What do you think could have made your GK12 experience better? The student answers were generally positive, of 136 completed TOSRA surveys, 99 students answered Q1, and 92 answered Q2 (Figure 6.3a,b). We used the process of grounded theory to identify themes among written answers (Pope et al., 2000), resulting in 9 themes in response to $\mathrm{Q} 1$, and 15 themes in response to $\mathrm{Q} 2$. In response to $\mathrm{Q} 1$, an overwhelming majority answered that they liked "Mosscosms" projects the best (46\% of 99 surveys that answered Q1), and the second most popular activity was ecology fieldwork (14\% of surveys that answered Q1). In response to Q2, the greatest 
response category was that they would not have changed anything ( $21 \%$ of 92 surveys) and the second most cited critiques were that students thought it could be "more interesting" (10\% of 92 surveys), closely followed by $9 \%$ of students who reported concerns with their own performance and efforts which is binned as "technical/personal issues".

\section{Discussion}

In summary, we believe that by bringing this globally relevant, novel, fits-inyour-palm moss ecosystem into the classroom, we provided a uniquely tractable, observable and repeatable system by which students could explore ecosystem function and, more broadly, start thinking in a multi-dimensional manner. Insight on student thought processes emerged not only in lab reports and poster analyses, but also throughout the progression. Research has shown that when students interact with a living system, interest and understanding of the subject increases (Eilam, 2012). Our students exhibited unexpected behaviors of responsibiity and ownership of the microcosms, for example, many individuals voluntarily came into the classroom during lunchtime and after school to check on their Mosscoms. A video clip developed on the project highlights students discussing their projects (http://www.youtube.com/watch?v=BJev48KpDxs).

Over time, many groups were faced with needing to reassess their understanding of the system and accept that their starting hypothesis may 
not even apply anymore. In the intermediate period, with little input from either teacher or scientist, when the groups observed, questioned and discussed what was happening in their mosscosms, is when we believe the true complex learning and engagement occurred. In some cases, springtail populations would boom, and in others all springtails would appear dead, mosses would turn brown, or become covered in microbes. Students then began to consider many components of the world they created, not just the factors they constructed their hypotheses around.

The majority of Mosscosm events were meticulously documented in lab notebooks, and refreshingly, mistakes were as well. In one case, upon watering her mosscosms, a student had accidentally grabbed a spray bottle of cleaning solution, not water, and given a few squirts to the mosscosm. This information came up as we tried to make sense of the unexpected appearance of thousands of mite eggs stuck to the edges of one Mosscosm. She promptly opened her notebook and figured out when the accident occurred and how much of the toxic application was applied. We were then able to make informed inferences about the strange happenings in that Mosscosm. This example illustrates students working as a scientist works, making connections and then moving forward from gained information.

The issue remains that it is difficult to assess the efficacy of science education without a formally evaluated assessment. As in scientific research, science education must have reliable quantitative data that allows for 
effective evaluation, and dissemination of gained information. As we could not foresee the success of the project, the only formal assessment conducted were TOSRA surveys and written answers to open-ended questions (Figs. 6.3 and 6.4). The written questions added a valuable component to the surveys, as the survey itself may have been a bit complicated for our youngest students ( $6^{\text {th }}$ grade), and the older students ( $8^{\text {th }}$ grade) voiced concerns about the redundancy of the questions. Some evidence supports that attitude surveys such as these can be difficult for respondents to interpret (Strack \& Martin, 1987) and may not yield reliable data (Schuman \& Presser, 1996), which must be taken into consideration when evaluating survey results.

In the future, when implementing this curriculum, we recommend a pre and post comprehension survey and/or analysis of student knowledge on basic concepts. An ideal assessment of this project would include the development of a contextual assessment of student understanding of basic broad ecological themes, experimental design and data analysis (for assessment examples see: (Brownell et al., 2014, Lisowski \& Disinger, 1991, Thornton \& Sokoloff, 1998) to be implemented before and after the project. Such assessment would allow educators to gauge student misconceptions, gaps in understanding and gained comprehension.

Although getting students to reflect upon complex system dynamics can be difficult to achieve (Grotzer et al. 2013) we deem the following student discussion excerpts do show complex thinking: 
I believe that the sugar water [treatment] having the highest population was due to the moss's absorption of the sugar water. I think that as the sugar water evaporated and then precipitated again the moss took in the nutrients from it. Therefore the moss remained fresh and green throughout the experiment and the springtails were able to thrive in the moss. This was an interesting possibility I had not considered when making my hypothesis. Sixth grade.

I think that not many springtails reproduced because their environment was too dry to be comfortable. I think I should have sprayed my bryosphere with water more often than one time per week. Also while I was observing the bryosphere I noticed lots of spiders. I did not add any spiders into my bryosphere but one or two might have crawled in while I was adding the moss in the beginning of the experiments, this might also be a cause of the low springtail populations, because the spiders might have been feeding on the springtails. Seventh Grade.

I thought that we had killed all of our springtails by giving them chocolate to eat - although at first I thought they'd like it. But then the chocolate grew lots of different bacteria and I thought it would kill them, but springtails like to eat Lindor chocolate's bacteria the best and we ended up with thousands of springtails that grew from a population of twenty! Sixth Grade.

We view the above student statements as indicative of multi-level thinking (Wilensky and Resnick 1999), and although students may or may not be interpreting data "correctly," the Mosscosm project allowed students to make connections between and among ecosystem components and exterior factors that may not have arisen if they were not intimately engaged in their own self-designed projects. We hope that this hands-on, moss-based biology project can be adopted, modified, evaluated, and bettered in more classrooms as it uniquely presents the opportunity for students to engage not only with 
singular organisms, but also with entire dynamic systems. As upcoming scientists, artists, doctors, investors, engineers, teachers, parents, and community members, our students deserve to learn in a way that is flexible, effective, and timely. Textbook-based, broadly practiced teaching methods may not reach all students, therefore leading to enhanced inequities in the classroom and beyond. As more educators invest in science teaching that is rooted in discovery and relevant research, students will hopefully gain transferrable skills and knowledge of concepts that are essential to our collective futures. 


\section{Tables and Figures}

Table 6.1. Student TOSRA Survey Results. Questions and results from TOSRA surveys administered to students involved in Cascades to Coast, GK12 in 2012-2013. Mean answer, standard error mean and number of answers are reported. The response scale is from 1-5 (1=Strongly Disagree, 2=Disagree, 3=Not Sure, 4=Agree, 5=Strongly Agree).

\begin{tabular}{|l|r|r|r|}
\hline Statement & \multicolumn{1}{l}{ Mean } & \multicolumn{1}{l}{ SEM } & \multicolumn{1}{c|}{ N } \\
\hline 1. I would not like to be a scientist after I leave school. & 3.33 & 0.089 & 135 \\
\hline 2. Science lessons are fun. & 3.39 & 0.074 & 135 \\
\hline $\begin{array}{l}\text { 3. When I leave school, I would like to work with people who } \\
\text { make discoveries in science. }\end{array}$ & 2.85 & 0.087 & 130 \\
\hline $\begin{array}{l}\text { 4. I dislike learning about science and the environment in our } \\
\text { community. }\end{array}$ & 2.35 & 0.074 & 133 \\
\hline $\begin{array}{l}\text { 5. I would not like a job in a science laboratory after I leave } \\
\text { school. }\end{array}$ & 3.36 & 0.097 & 135 \\
\hline $\begin{array}{l}\text { 6. I would like to have the opportunity to learn more science } \\
\text { lessons each week. }\end{array}$ & 2.88 & 0.093 & 132 \\
\hline $\begin{array}{l}\text { 7. Working in a science laboratory would be an interesting } \\
\text { way to earn a living. }\end{array}$ & 3.27 & 0.087 & 135 \\
\hline 8. Science lessons bore me. & 2.97 & 0.101 & 135 \\
\hline 9. A career in science would be dull and boring. & 2.69 & 0.099 & 133 \\
\hline $\begin{array}{l}\text { 10. Learning science and about my community environment is } \\
\text { a very interesting subject. }\end{array}$ & 3.25 & 0.084 & 134 \\
\hline 11. I would like to teach science when I leave school. & 1.99 & 0.087 & 134 \\
\hline 12. Science lessons are a waste of time. & 2.02 & 0.089 & 134 \\
\hline 13. A job as a scientist would be boring. & 2.63 & 0.093 & 136 \\
\hline $\begin{array}{l}\text { 14. I really enjoy learning about science and the environment } \\
\text { in our community. }\end{array}$ & 3.17 & 0.083 & 133 \\
\hline $\begin{array}{l}\text { 15. A job as a scientist would be interesting } \\
\text { environment. I look forward to learning more about science and the }\end{array}$ & 3.15 & 0.085 & 132 \\
\hline
\end{tabular}




\begin{tabular}{|l|r|r|r|}
$\begin{array}{l}\text { 17. I would not like to become a scientist because it needs too } \\
\text { much education. }\end{array}$ & 2.5 & 0.09 & 135 \\
\hline $\begin{array}{l}\text { 18. I would enjoy myself more if we did not have to learn } \\
\text { about science. }\end{array}$ & 2.5 & 0.088 & 131 \\
\hline 19. I would like to be a scientist when I leave school. & 2.34 & 0.085 & 134 \\
\hline $\begin{array}{l}\text { 20. The material we are learning about science is not } \\
\text { interesting to me. }\end{array}$ & 2.57 & 0.094 & 133 \\
\hline $\begin{array}{l}\text { 21. I go outdoors to natural environments in my free time } \\
\text { whenever I can. }\end{array}$ & 3.2 & 0.092 & 132 \\
\hline $\begin{array}{l}\text { 22. I think you can get along fine without spending time in } \\
\text { nature. }\end{array}$ & 2.3 & 0.088 & 134 \\
\hline $\begin{array}{l}\text { 23. I like to learn about the plants and animals that live in } \\
\text { the environment around me. }\end{array}$ & 3.42 & 0.088 & 134 \\
\hline $\begin{array}{l}\text { 24. I can recognize when the natural world around me is not } \\
\text { healthy -- like polluted rivers. }\end{array}$ & 4.08 & 0.231 & 133 \\
\hline $\begin{array}{l}\text { 25. I think people can do helpful things for nature and the } \\
\text { natural world. }\end{array}$ & 4.4 & 0.066 & 132 \\
\hline $\begin{array}{l}\text { 26. I know a lot about the regional environment here - the } \\
\text { area that goes from the Cascade Mountains to the Oregon } \\
\text { Coast. }\end{array}$ & 2.95 & 0.086 & 131 \\
\hline $\begin{array}{l}\text { 27. I am aware-of how I can affect the health of the natural } \\
\text { environment that is around me. }\end{array}$ & 3.94 & 0.069 & 134 \\
\hline $\begin{array}{l}\text { 28. I am interested in having a career working to help the } \\
\text { environment when I grow up. }\end{array}$ & 2.69 & 0.086 & 133 \\
\hline $\begin{array}{l}\text { 29. I talk to my parents and friends about the regional } \\
\text { environment here - the area that goes from the Cascade } \\
\text { Mountains to the Oregon Coast. }\end{array}$ & 2.35 & 0.091 & 134 \\
\hline $\begin{array}{l}\text { 30. I actively advocate for taking care of the natural world } \\
\text { plants and animals) by talking to others. }\end{array}$ & 2.88 & 0.092 & 132 \\
\hline
\end{tabular}


Figure 6.1. Student-generated springtail food choice results. Student data from year one on springtail population growth over five months on three food sources in a controlled habitat. A population increase was found when springtails were fed yeast (+92 springtails), and a population decrease was found when springtails were fed a moss piece (-80 springtails), little change was found when springtails were fed a rice diet (+26 springtails). Results of a Two-Way ANOVA are non-significant (Column Factor, Food Source, $\mathrm{P}=0.367$; Row Factor, Month, $\mathrm{P}=0.82$ ) due to low sample size, but results indicated that yeast may the best represented food source for springtail population growth in an artificial habitat.

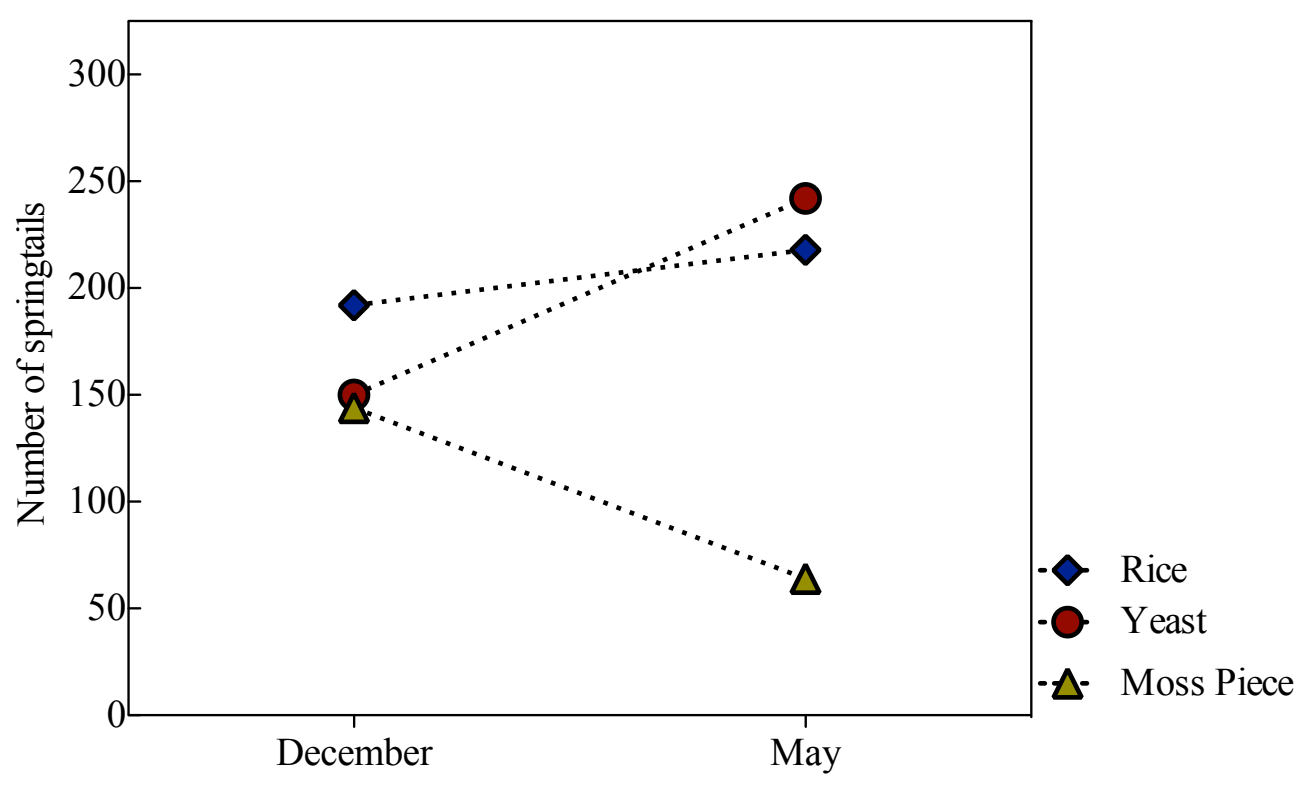


Figure 6.2. Results of TOSRA surveys. Response scale from 1 to 5 (1=Strongly Disagree, $2=$ Disagree, $3=$ Not Sure, $4=$ Agree, $5=$ Strongly Agree). Student responses to questions 1-20 regarding 'enjoyment and interest in a career in science.' The solid white bar represents student attitude responses questions with positive connotations and the patterned bar represents responses to questions with negative connotations. $(\mathrm{P}<0.003 ; \mathrm{N}=136$; Bars represent mean \pm SEM).

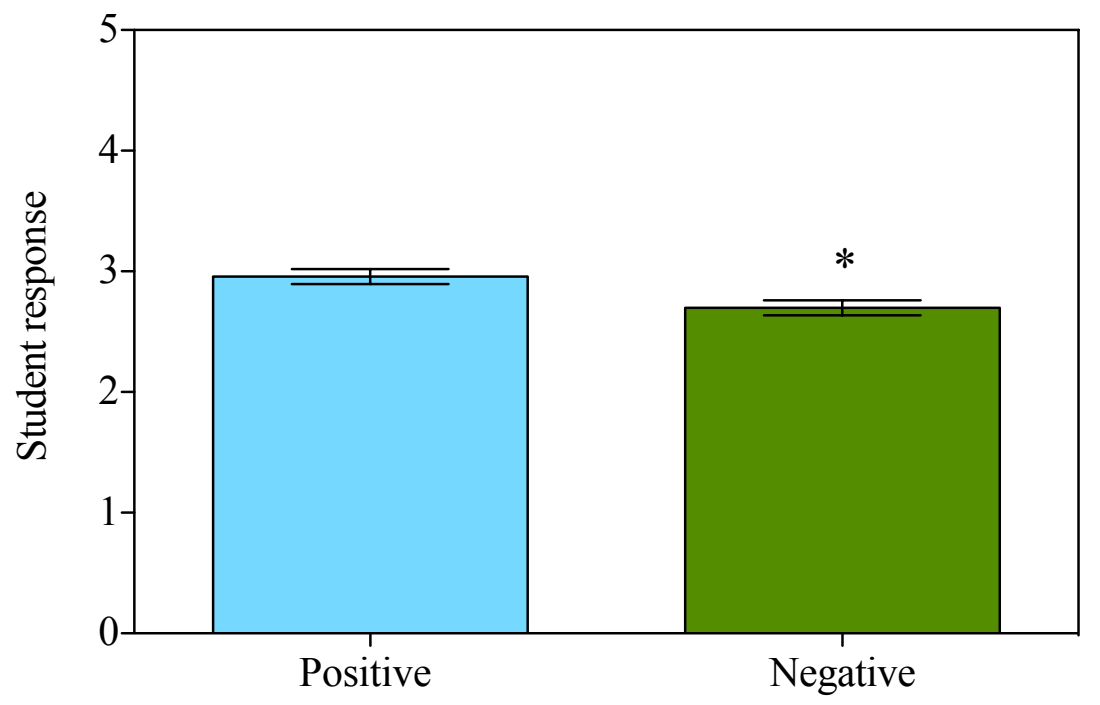


Figure 6.3 Student responses to open ended questions. a) Student responses to written Question 1 (Q1): "What was the your favorite GK-12 activity and why?" Of 136 surveys administered, 99 students answered Q1, nine themes were identified. b) Student responses to written Question 2 (Q2): “What could have made your GK-12 experience better?" Of 136 surveys administered, 92 students answered Q2, fifteen themes were identified. 


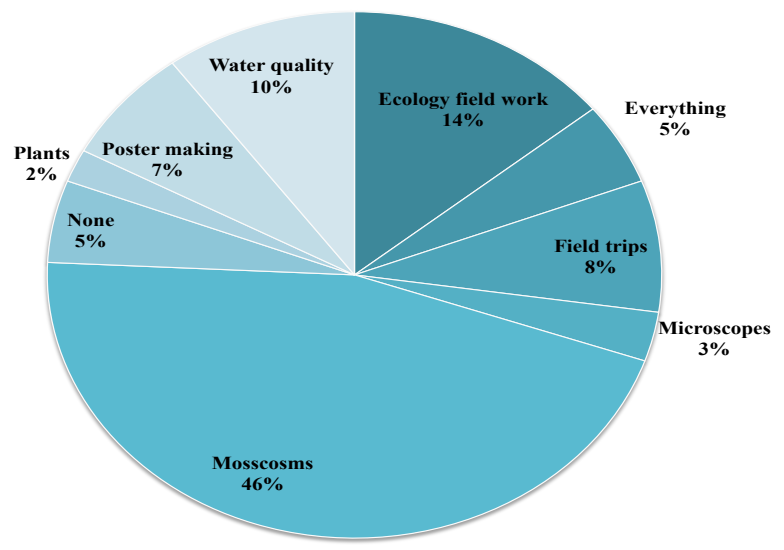

b

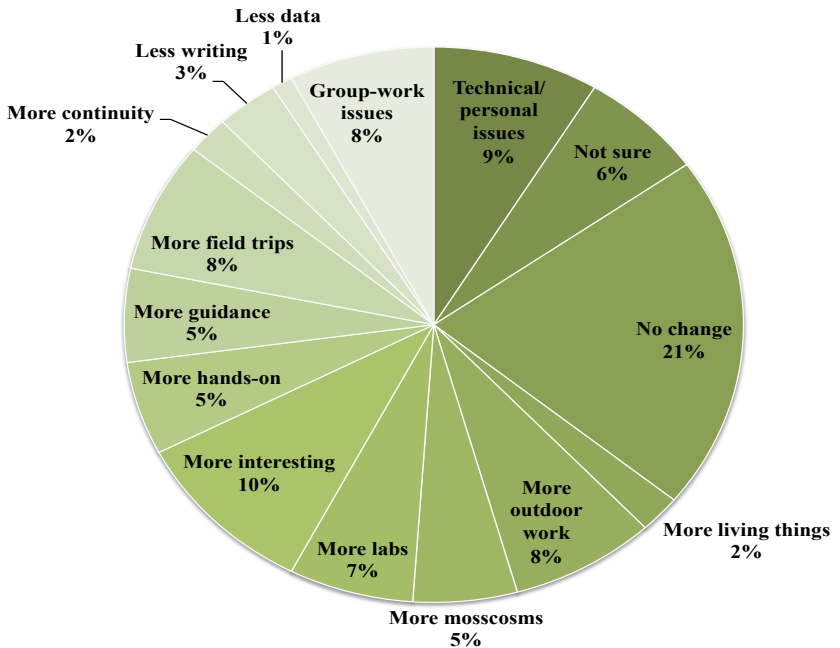

$5 \%$ 


\section{Chapter 6}

\section{Supplementary Materials}

Mosscosms: A Research-Based Activity to Encourage Ecological Thinking in the Classroom

\section{S6.1. National Academies and Oregon State Standards met in} Mosscoms project: National Academy of Sciences Framework for K-12 Science Education for Life Sciences Core National Academies, (Quinn et al., 2012)

- The first core idea, LS1: From Molecules to Organisms: Structures and Processes, addresses how individual organisms are configured and how these structures function to support life, growth, behavior, and reproduction. The first core idea hinges on the unifying principle that cells are the basic unit of life.

- The second core idea, LS2: Ecosystems: Interactions, Energy, and Dynamics, explores organisms' interactions with each other and their physical environment. This includes how organisms obtain resources, how they change their environment, how changing environmental factors affect organisms and ecosystems, how social interactions and group behavior play out within and between species, and how these factors all combine to determine ecosystem functioning. 
- The third core idea, LS3: Heredity: Inheritance and Variation of Traits across generations, focuses on the flow of genetic information between generations. This idea explains the mechanisms of genetic inheritance and describes the environmental and genetic causes of gene mutation and the alteration of gene expression.

- The fourth core idea, LS4: Biological Evolution: Unity and Diversity, explores "changes in the traits of populations of organisms over time" [1] and the factors that account for species' unity and diversity alike.

\section{Oregon State Standards (Oregon Department of Education, 2009)}

Interaction and Change:

- The related parts within a system interact and change (6.2)

- The components and processes within a system interact (7.2)

- Systems interact with other systems (8.2)

Scientific Inquiry:

- Scientific inquiry is the investigation of the natural world based on observations and science principles that includes proposing questions or hypotheses, and developing procedures for questioning, collecting, analyzing, and interpreting accurate and relevant data to produce justifiable evidence-based explanations. (6.3)

- Scientific inquiry is the investigation of the natural world based on observations and science principles that includes proposing questions or hypotheses, designing procedures for questioning, 
collecting, analyzing, and interpreting multiple forms of accurate and relevant data to produce justifiable evidence-based explanations. (7.3)

- Scientific inquiry is the investigation of the natural world based on observations and science principles that includes proposing questions or hypotheses and designing procedures for questioning, collecting, analyzing, and interpreting multiple forms of accurate and relevant data to produce justifiable evidence-based explanations and new explorations (8.3)

\section{S6.2. Scaffolding}

\section{A. Ecological Sampling Techniques and Environmental Surveying}

\section{(2-3 days).}

- Through discussion and outdoor activities introduce the importance of random sampling/subsampling to gain an idea of the environment or ecosystem's characteristics/inhabitants.

- Plants: Use transects and quadrats to count plant species richness and abundance around the school yards - discuss why scientists would use such techniques

- Collect abiotic data surrounding sampling sites

- Compile all class data 
- Have students draw a data table on the board and take average values - discuss why to use averages, what is "error"?

- Graph the data by hand on the board, introduce computer graphing

- Discuss the data and interpret results

- How might these data results be different in a forest? Farmland? At their houses?

\section{B. Water Cycle and Water Quality ( 1 week)}

- Basics of the Water Cycle with an emphasis on the "never-ending", cyclical journey of water

- Focus on water quality metrics and technology used to collect environmental data.

- We use Vernier Software \& Technology LabQuest interface and sensors (Beaverton, OR)

- Turbidity, Total Dissolved Solids (Conductivity), $\mathrm{pH}$

- What effects or changes these quality metrics? (sources)

- What impacts does a change in various water quality metrics have?

- Conduct first hands-on guided-inquiry activity by testing "unknown" water sources and have students make predictions about where the waters came from and why they think so

\section{Photosynthesis (1-2 days)}


- Students visit or hopefully revisit the basic concepts of photosynthesis with Powerpoint slides, handouts, live plants, and discussion

\section{S6.3. Mosscosms Scaffolding}

\section{A. Ecology basics (1 day) - use mixed media presentations, pictures and discussion}

Students discuss and learn about biotic and abiotic factors and how they interact to form an ever-changing ecosystem with all domains of life and environmental influences.

B. Bryospheres (2 days) -Introduce the formal scientific definition from the peer-reviewed journal article by Lindo and Gonzales (2010, Ecosystems). Students then form their own definitions based on a classroom conversational dissection of the formal definition.

- Who are the main players of the bryosphere?

- Focus on mosses and microarthropods and ecosystem-wide interactions

- What do they need to survive?

○ Mosses

- Life history traits

- They need: CO2, H20, Sunlight, substrate

- Habitat preferences

○ Microarthropods: 
- Springtails, mites, nematodes, tardigrades, arachnids, worms

- Life history traits, habitat

- Food sources

- Show clips of the BBC's David Attenbourough documentary, "Life in the Undergrowth." (The springtail footage was very popular and allowed student to forge a familiarity and awe with the microarthropods)

- How might these organisms interact in the bryosphere? Who else is there? What abiotic and biotic factors affect the system?

- Demonstrate how one can use a simple extraction method to find out who lives in moss patches or leaf litter (modified Berlese Funnels (Hopkin, 1997))

- Look at collected microarthropods under the microscope

\section{Experimental Design}

Through a class vote students determine what the general subject of the testing will be (i.e. testing moss health or springtail fecundity) in their classroom.

- Classroom discussion and presentation follows concerning:

- Introduction into sound experimental design to answer specific questions. What will be controlled and what will be your dependent and independent variables? 
- What is the difference between general observations of a system and testing a specific question? Can you do both at once?

- What might the data look like upon collection? Students guide each other through graphing of hypothetical data on the board as a class.

- How may data collection commence? How often will you collect data? What treatments will be applied, how often and by whom? Will your data collections answer your research questions?

- Teacher and scientist provide sample lab-notebook data entries and observations ranging from "fair" to "excellent".

\section{S4. Mosscosms Research proposal}

\section{A. Develop proposal}

- What is the research question?

- Develop specific hypotheses. (Does the experimental design directly test the hypothesis?)

- Identify the independent/dependent variables.

- Determine the desired data to be collected to answer the research question.

- Identify potentially confounding variables.

- Make a materials list. 
The proposal is written and drawn up informally, but articulated by each group to the panel (teacher and scientist) for review. The panel then either approves the proposal immediately or asks the group to meet to make suggested amendments to proposal for re-evaluation.

\section{S6.5. Examples of Student Mosscosm Research Questions}

- How does a change in $\mathrm{pH}$ affect a mosscosm?

- How do springtail populations change if predators are added?

- How do springtail populations change with different species of predators?

- How do elevated $\mathrm{CO}_{2}$ levels affect mosses-microarthropod mosscosms?

- How does a given amount of food change springtail population growth?

- If you add scents to mosses, do the springtails prefer one moss over another?

- How do different light and humidity levels affect the health of a $\operatorname{mosscosm} ?$

- How does a "natural" vs. "artificial" habitat change springtail behavior?

- Does moss species diversity change mosscosm dynamics?

- Does one moss species provide a better habitat for springtails than another?

\section{S6.6. Example student material list}


Group: Moss is Boss

Research Question: What are the effects of 4 different light levels on springtail populations living in moss?

- 4 large mason jars with lids

- Black cloth

- Moss (enough of the same moss for all mosscosms)

- Clip light and light bulb

- Ring stand

- Yeast grains

- Popsicle sticks

- Balance or scale

- Masking tape

- 40 springtails (10 for each mosscosm)

- Spray water bottle

Useful website for ordering springtails (Folsomia candida):

www.joshsfrogs.com

Useful website for ordering mosses: www.mossacres.com

\section{S6.7. Student responsibilities}

- Decide what data is to be collected. Who does what and when?

- Make an observation/data collection schedule. 
- Maintain observations/data collection/treatments at least $1 \mathrm{x}$ per week, adapt as you see fit

- Allow experiment to run for at least 3-4 months, giving time for springtail reproduction and other ecosystem dynamics to occur.

- Write down anything you notice, whether it seems relevant or not!

\section{S6.8. Sample lab notebook entry}

Group name: Moss is Boss

Date: 02.20 .2013

\section{Treatments made:}

- All jars were opened for gas exchange

- Mosscosm 1,3,4 sprayed with squirt bottle of tap water 4x each

\section{Observations:}

- Mosscosms 1 and 3 (ambient light level): have a few obvious springtails running around (1 more than 3 ), some bacteria, moss looks ok, pretty green, they could use a bit more water

- Mosscosm 2 (no light) - I see some springtails, pinkish and greenish bacteria or fungi, moss looks browner, we should water it less (I did not water today), it may be dying with no light...

- Mosscosm 4 (high light): Has no springtails! I think they are dead and the moss looks brownish-yellow. Maybe we should water it more. 
S6.9. Steps for collecting Mosscosm data, analysis, and concluding activities

\section{A. Data Collections/Harvest Experiment}

- Determine what data to collect.

- How will you collect data? Is it appropriate to answer your questions?

- What do you want the data to look like in a table/graph?

- In what order should the data be collected?

- Is there any additional information that you can gain before finishing?

- Write-out your proposed data collection process and necessary materials

- Run the above information by your instructors for approval/suggestions

- Collect data (harvest experiment)

- Save springtails and all organic materials that have not been overly manipulated (i.e. too much bacterial/fungal growth - throw these away!)

\section{B. Results, Data, Data Analysis, Lab Report}

- Enter data into excel spreadsheet

- Determine how you want to analyze data

- Graph data in Excel

- What do the results tell you?

- Can you explain your results? 
- Think about how to discuss your results in a broader context.

- What new questions did your experiment bring up?

- What would be your next steps to follow up or support your experiment?

\section{Key components of a formal lab report:}

Introduction, Materials and Methods (detailed), Data Table(s), Data Figures, Results, Discussion

\section{Make a scientific poster:}

- Reread your lab-report and your lab notebook - think about how to tell your experiment in a story

- What needs to be on the poster? Everything from your lab report, but differently!

- Minimize words - make succinct statements instead of sentences in all but the introduction and the conclusion (if those!)

- Be creative, tell a story

- Make it visually interesting, yet logical and easy for the viewer to understand.

- When can you use a picture or drawing instead of words?

- Remember, people may only have a few seconds to look at your poster so:

- What do you most want people to know - what is the take-home message? 
- What is unique about your study? What were you testing? What did you learn? What are the next steps?

\section{Video}

V6.1. Cascades to Coast and Portland State University Present: da Vinci Arts Middle School GK-12 Website: http://www.pdx.edu/soe-gk12/videos-and-minidocumentaries:

YouTube: http://www.youtube.com/watch?v=BJev48KpDxs 


\section{Chapter 7}

\section{Discussion}

By considering closely what we already know about the bryophytes, it becomes clear that there are still many unanswered questions about their biology. Taken together, the studies outlined in this dissertation highlight the complexity of what lies within the often-overlooked mosses, and significantly contributes to the field of plant biology by uncovering previously unknown forces shaping moss reproductive biology. As a phylum, mosses are incredibly successful and abundant. Over a number of Paleozoic periods mosses made their way out of the water and across every continent. The crux of any organism's success lies in its ability to reproduce, thus highlighting the need for in-depth study on the functional biology, physiology and ecology of moss reproduction at both an experimental and ecological level.

\section{Experimental work}

Moss reproduction of today preserves relics of its ancient aquatic origins by requiring that free-motile flagellated sperm travel ex planta to a female moss for fertilization to occur (Glime, 2007, Goffinet \& Shaw, 2009, Ligrone et al., 2012). I begin my dissertation work by challenging the accepted dogma of male moss sperm limitation by water availability (Longton, 1976, Wyatt \& 
Anderson, 1984). Stress tolerance is a key, ongoing dilemma for mosses, (Bewley et al., 1974, Goffinet \& Shaw, 2009, Oliver et al., 2000), thus providing the rationale to test all of a moss's biology in the realm of stress. Stress tolerance in moss sperm has been tested in a heat-tolerant monoicous species with results indicating that the stress-tolerant mosses can maintain long-lived, heat tolerant sperm (Rosenstiel \& Eppley, 2009). Since water stress is the most prevalent and widespread of all abiotic stressors on mosses (Proctor et al., 2007), I tested whether the swimming sperm of water stress tolerant mosses also maintain water stress tolerance ex planta, once released from the antheridia. I tested the sperm of three moss species with separate sexes (dioicous) against environmental desiccation events. Our data show that a fraction of the moss sperm cells from each of the three species $(\sim 17 \%$ on average) show tolerance to environmental desiccation (Shortlidge et al., 2012). This seemingly constitutive trait is variable within and among species and populations (Figs 2.3 and 2.4), and the tolerance is increased when the sperm cells are dried down in a membrane stabilizing sucrose solution (Figs. $2.2,2.4 \mathrm{c})$. Not only is sucrose a key compound in facilitating intracellular desiccation tolerance across taxa (Crowe et al., 1992, Koster, 1991, Smirnoff, 1992, Sun et al., 1994), but sucrose is a known constituent of female moss archegonia (Kaiser et al., 1985, Ziegler et al., 1988) as well as a pulse released sugar from permeable moss tissues during desiccation events (Coxson et al., 1992, Wilson \& Coxson, 1999). These data suggest that perhaps the sperm 
cells have evolved the capacity to prevail even if water is not always present and could take advantage of moss-generated environmental carbohydrate protection. Testing whether or not these cells themselves are capable of successfully fertilizing the female mosses after stress events remains unknown, and if they cannot, what purpose do they serve in the context of moss reproductive evolution? This system presents intriguing topics for cell biology research concerning one of the most basal examples of out-of-thewater eukaryotic motile male gametes.

The previously unconsidered role for stress tolerant moss gametes on the landscape begs questions regarding the potential implications for the phenomenon. Why would "swimming" sperm evolve tolerance to environmental desiccation unless there was a practical (fitness) application? Perhaps these gametes survive ex planta on the landscape, as for example, a pollen grain does. Pollen grains are carried to female recipients by abiotic and biotic forces such as wind and pollinators (e.g. flying and land-travelling invertebrates, birds and mammals) (Lord \& Russell, 2002). We wanted to know: if moss sperm that are released onto the landscape are stress tolerant, are they also being moved among mosses by vectors other than water? In many locales that mosses inhabit, water can be quite ephemeral, yet sporophytes are often widespread (Longton, 1976, Wyatt \& Anderson, 1984). To test this idea we built on previous work performed by Cronberg et al. (2006) where the researchers showed in an elegant yet very simple 
experiment that in the presence of mites and Collembola, diocious mosses in Petri dishes develop more sporophytes, (the matured product of successful fertilization) than without the microarthropods. Further, when the male and female moss patches were separated by either two or four centimeters, the sporophytes were only formed in the presence of the microarthropods and more so with Collembola (springtails) over mites. These experiments also showed that the microarthropods preferentially visited mosses that were expressing sex organs. In order to further test the idea of microarthropods acting as gamete transfer vectors, we took a three-pronged comprehensive approach to test the major tenets of plant-pollinator theory on this basal land-plant system.

The results of the study are described in Chapter 3, Sex specific volatile compounds influence microarthropod-mediated fertilization in mosses (Rosenstiel et al., 2012). Here, we tested the relative effects of the presumed abiotic (water) and biotic (microarthropod) vectors for sperm transport in moss microcosms using a ubiquitous model moss system, Ceratodon purpureus. We found that not only do both vectors induce fertilization in mosses, but in accordance with Cronberg et al. (2006), in the presence of microarthropods alone more sporophytes are produced than just water or no vector treatment, and as one might intuit, the two vectors are combined elicit a significant synergistic effect yielding the greatest number of sporophytes per microcosm (Fig. 3.4). 
In Chapter 3 we also present the novel finding that mosses emit sexspecific volatile compound suites (similar to flowering plant floral scent "bouquets"). The volatile organic compounds (VOCs) were collected from the headspace of intact, sexually mature male and female clones (of controlled biomass) using solid-phase microextraction (SPME) fibers followed by twodimensional gas chromatography (GCxGC) analysis. This comprehensive analytical technique allows for identification of many compounds as well as a finer peak resolution than would otherwise be obscured by one dimensional GC analysis (Pankow et al., 2011, Perera et al., 2002). On average, 104 compounds were identified from female C. purpureus mosses and 30 from males, revealing significant sex-specific differences in total number, magnitude and suite of identified VOCs (Figs 2.1, 2.3, A.A1-3). We also found that Bryum argenteum females emitted more VOCs on average than males of the same populations (Fig. A.A1), but differences were not significant likely due to small sample size and significant variation among individuals. $B$. argenteum is also a common dioicous weedy moss.

Overall, the VOC suites emitted from the female moss canopies were more complex, greater in quantity, and generally more similar in composition to one another than those detected coming from male canopies (Figs 3.1, 3.2, A.A1-3). Sex-specific VOCs have been detected in limited species of flowering plants, almost all of which are at least in part biotically pollinated, as reported in a review by Ashman (2009). In these studies, males often 
invest more into scent production, presumably to ensure enough pollinator visitations to get their genes out (Bell, 1985), yet significant pollen limitation has also been found in flowering plants (Ashman et al., 2004, Burd, 1994). Unlike in the flowering plants where only $\sim 6 \%$ are truly dioecious (Barrett, 1990, Renner \& Ricklefs, 1995), dioicy is historically prevalent and continuously evolving in the modern relatives of mosses (McDaniel et al., 2013a) with about $60 \%$ of mosses maintaining dioicy. If we look to the views of angiosperm and broadcast spawner reproductive system theory, we could predict that the female dioicous mosses are the mate-limited sex (spermlimited) due to sperm diffusion rates and distance from female (Levitan \& Petersen, 1995) and therefore would invest more into reproductive assurance such as through the use of biotic dispersal agents (Ashman, 2009).

The males produce thousands of sperm, thus it is hypothesized that their reproductive efforts are realized by mass production of gametes (Stark et al., 2000). Further, researchers found that in B. argenteum, (the other tested for sex-specific VOCs) has shown that males exhibit higher prezygotic reproductive investment than females in the form of sex expression (sperm production) (Horsley et al., 2011) and similar traits were revealed in $C$. purpureus (Shaw \& Beer, 1999). Thus, the researchers proposed that male investment costs into reproductive assurance are higher in dioicous mosses than in females, particularly in a female biased landscape (Stark et al., 2000), while other researchers do not find evidence to support the higher male cost 
hypothesis (Bisang et al., 2006). If the males are investing heavily into gamete production it is also likely that adaptations have evolved to minimize the severity of sperm limitation (Yund, 2000), by mechanisms such as spermcompetition, stress tolerance, and dimorphic sperm (Buckland-Nicks et al., 1999, Gou et al., 2009, Lee \& Wilkes, 1965, Rosenstiel \& Eppley, 2009, Shortlidge et al., 2012, Till-Bottraud et al., 2005) as discussed in detail Chapter 2. Yet, all of these findings may be consistent with one another in supporting the sperm limitation hypothesis if we consider the notion that female and male investment strategies differ significantly from each other due do each sex's respective challenges and perhaps the female investment strategies are directed in previously untested ways such as recruitment (e.g. scent, biotic mutualisms).

In flowering plants, a mate-limited state often leads to the recruitment of biotic pollinators, along with abiotic vectors (e.g. wind and water) and this occurs in all mating systems of flowering plants to some extent, generally to avoid inbreeding depression (Charlesworth \& Charlesworth, 1999). Whether this is true for the mating systems in mosses remains relatively untested although researchers found that monoicous mosses, unlike many other organisms, do not suffer from inbreeding depression as dioicous mosses do (Eppley et al., 2007, Jesson et al., 2007, Szövényi et al., 2014). In Appendix B, Testing biotic and abiotic influences on the reproductive biology of the monoicous moss, Physcomitrella patens, I discuss how I tested a monoicous 
moss's relationship to microarthropods and found no evidence of increased fertilization or outcrossing with the addition of Collembola. Yet these experiments could be improved upon and repeated with other, more common species, and testing for volatile compound emission from monoicous species would be key to further investigating a relationship between microarthropods and monoicous moss species.

In angiosperms, evidence of increased investment towards biotic pollinators can come in the form of volatile scent production as well as in rewards (Ashman, 2009, Dudareva \& Pichersky, 2010, Raguso, 2008). Such ideas could explain our findings of marked sex-specific differences in VOC suites influencing Collembolan preferences towards female mosses, yet the potential rewards remain unknown. Our preference assays show Collembola repeatedly choosing to visit female mosses: both in visiting the entire intact canopy and when they are isolated from the physical mosses, being guided by chemical cues alone (Fig 3.3). These data were the first to demonstrate empirical support for a complex scent-based relationship between the mosses and their microarthropod inhabitants, but it only opens the door for more investigation and interpretation. One aspect of these scent data that remains unresolved is which scent cues the Collembola are responding to, and from where exactly they are originating. We analyzed the head-space volatile suites of intact male and female moss canopies, and as with any naturally occurring plant system, the moss canopy is rife with microbial inhabitants 
(Davey et al., 2012, Kachalkin et al., 2008, Opelt et al., 2007). There are a number of possible explanations for the Collembolans preferring female mosses, including the obvious option of food source. Even if the Collembolan choices are due to attraction towards a food source living on the mosses, the food source itself is associated with the female mosses. Our lab group is investigating the origins of these cues in order to begin unraveling the functional biology behind these novel findings.

For a relationship to be a "syndrome," selection must be acting on the maintenance of the mutualistic interactions, presumably providing positive feedback and reciprocal resources or benefits (Fenster et al., 2004). It is known that microarthropods eat microbes and detritus that live on and among the mosses, but in general, do not feed on the moss tissue itself (Block, 1985, Hopkin, 1997, Klironomos et al., 1992, Sadaka-Laulan et al., 1998, Walter, 1987), which implies that the mosses are not particularly palatable (Jennings \& Barkham, 1975) with the exception of immature protonema and sporophyte capsules (Davidson et al., 1990). Mosses do provide a protected habitat for numerous microarthropods as well as a passing and feeding ground for many other invertebrates (Block, 1985, Gerson, 1969, Glime, 2007). Although not empirically tested, bryologists historically and currently suggest that invertebrates are eating the lipid-sperm masses and/or the archegonial extracts excreted from sexually mature male and female mosses (Cronberg, 2012, Gibson \& Miller-Brown, 1927). Such tests would certainly 
broaden our knowledge of what more the mosses provide for their common invertebrate guests other than habitat. It is also possible that the exudates are not themselves the food source, but provide a specific habitat, food source, or substrate for microbial life, that in turn lures in the microarthropods. These tests would be relatively easy to execute via both simple controlled observational behavioral tests such as olfactometer preference assays (Bengtsson et al., 1991, Klironomos et al., 1992, Rosenstiel et al., 2012), and in more complex multi-trophic isotopic labeling experiments (Churchland et al., 2012, Krab et al., 2008, Ostle et al., 2007).

Perhaps female mosses are recruiting invertebrates to visit them and along the way, they pick up one of the thousands of possible moss sperm on the landscape, thus diminishing the severity of sperm limitation in female mosses. Alternately, the relationship could be less haphazard and more akin to specialized co-evolved plant-pollinator relationships (Fenster et al., 2004, Schiestl, 2010) where specific differential traits emerge in both the plant and the invertebrate (Johnson \& Steiner, 2000, Knudsen \& Gershenzon, 2006). If the traits promote reproductive success or superior progeny, selection will act on those heritable traits (Bawa, 1990). The only other study of a specific moss-invertebrate scent-based mutualism is also a dispersal story where researchers describe how flies are essentially tricked into dispersing dung moss spores by chemical mimicry (Marino et al., 2009). Presumably there are many more examples of unique moss-invertebrate mutualisms given the 
prevalence of their long-term co-occurrences worldwide (Glime, 2007) that simply have not yet been uncovered. Direct evidence of payoff from an investment in mutualism by mosses could come in the form of increased genetic diversity in mosses as a result of microarthropod-mediated gamete dispersal, which is the presumed benefit for plants involved in pollination syndromes (Barrett et al., 1996, Bawa, 1990, Knight et al., 2005).

In ongoing work, we are testing the moss side of a potential mossmicroarthropod mutualism using experimental moss mesocosms deemed "bryospheres" (Lindo \& Gonzalez, 2010) as described in Appendix C, Testing mutualism theory in two of Earth's most ancient lineages: the mosses and microarthropods. My preliminary data further confirm previous findings that adding invertebrates to dioicous mosses increases sporophyte yield (Cronberg et al., 2006, Rosenstiel et al., 2012). Outdoor C. purpureus bryospheres received additions of full natural populations of moss invertebrates and we found significantly greater sporophyte yields in these bryospheres than without invertebrate additions (Fig. A.C3). Further, we find that individual male groups father more sporophytes than others when in the presence of microarthropods (Fig. A.C4), indicating variation in male moss fitness traits. Such variation presents a platform by which to empirically test co-evolution between the mosses and the microarthropods as well as genetically based sexually dimorphic traits (McDaniel et al., 2013b). If morphologic or metabolic traits belonging to male or female mosses 
differentially facilitate sperm dispersal and fertilization mediated by microarthropods in turn procuring benefits through increased dispersal and/or genetic variation, then a fitness benefit for the mosses is present. Taken together we have sufficient empirical evidence to support the hypothesis that mating systems in dioicous mosses are worthy of more indepth theoretical and experimental consideration, possibly shedding light upon the early evolution and alternate trajectory of plant-insect relationships.

\section{Moss biology and ecology in the field}

I tested aspects of moss reproductive biology on the cellular, metabolic and experimental microcosm levels. As any ecologist knows, nature is harder to assess, test, and interpret than controlled environments, yet can yield unparalleled insight (Southwood \& Henderson, 2009). In this section of Chapter 7, I discuss field studies (Chapters 4 and 5) testing aspects of community ecology, ecophysiology, and reproductive biology in moss-

dominated landscapes. In order to understand the biology of one organism, it is beneficial to investigate the messy functional ecology of its biotic and abiotic existence while in its natural habitats (Bruno et al., 2003, Pickett et al., 1992, Wright \& Jones, 2006).

Limited water availability is not the only environmental stress that mosses regularly encounter. Given their widespread abundance, it is inevitable that mosses experience a range of environmental stress. Sexual 
reproduction is altered or limited by extreme conditions in most organisms including the stress-tolerant mosses (Eppley et al., 2011, Grishkan et al., 2002, Kis-Papo et al., 2003, Lively et al., 1998, Stark et al., 2005). Few studies have assessed moss communities at high temperatures even fewer have asked questions about the moss-microarthropod relationship in geothermal regions (Convey \& Lewis Smith, 2006, Downie et al., 2000, Elmarsdottir et al., 2003, Fraser et al., 2014), and none to my knowledge have looked outside of the extreme peripheral (Iceland and Antarctica) geothermal systems.

To test how microarthropods might interact with mosses in a biotically reduced system I utilized an existing thermal gradient in an extreme environment (Lassen Volcanic National Park, (LVNP)) to test how biotic and abiotic factors influence a geothermal bryophyte system in regards to community members and moss genetic diversity. Studies divulge gene-toenvironment connections as well as environmental gradients as drivers of community-level function (Johnson \& Stinchcombe, 2007, McGill et al., 2006, Whitham et al., 2006). Previous research found that sex in mosses is limited at thermal extremes with few species producing viable sporophytes at temperature extremes of either direction (Convey \& Smith, 1993, Eppley et al., 2011, Ochyra et al., 2008). In Chapter 4, Community ecology of geothermal mosses: what drives diversity at temperature extremes?, I describe an ecology experiment designed to test in situ genetic variation of a common 
moss as it relates to microarthropod abundance. I found that even in a limited biotic region, there is great complexity working among the players of the moss ecosystem and that they vary along a geothermal gradient. We found a distinct decrease in invertebrate group diversity (Gini-Simpson Index) (Fig. 4.) with increasing temperatures. These results are in concert with microarthropod surveys where various environmental and ecological gradients drive species distributions and abundances (Adams et al., 2006, Borcard \& Legendre, 1994, Klironomos \& Kendrick, 1995). Although our coolest site (yet still geothermal) had the highest invertebrate diversity, as we would have expected, our site in the center of the thermal gradient was a hotspot for biological activity. At this particular site, (Site 3) the overall invertebrate abundance, fungal load, soil organic matter, and soil moss tissue nutrients were the greatest (Table 4.3 Figs 4.3-5). The sheltered and environmentally buffered complex of microbes and microarthropods within the moss canopies contribute significantly to biogeochemical cycles, decomposition and soil respiration rates (Lindo \& Gonzalez, 2010, Oechel \& Van Cleve, 1986, Turetsky, 2003, Turetsky et al., 2012). Given the consistency of these data, this particular moss-dominated community could yield insight into where the thermal line of biodiversity and activity lies. Also at this bioactive Site 3, was the greatest Collembolan abundance. Collembola are often linked to soil $\mathrm{C}$ and $\mathrm{N}$ dynamics, in particular to inorganic $\mathrm{N}$ availability as well as associated plant tissue $\mathrm{N}$ (Bardgett \& 
Chan, 1999, Osler \& Sommerkorn, 2007), and our data supports these findings of Collembolan abundance positively correlating with system N. As discussed previously, Collembola are identified as engaged in a pollinator-like relationship with the mosses, specifically with C. purpureus (Rosenstiel et al., 2012). At this same active site, dominated by Collembola, we have the highest within population genetic variation. Although sexual reproduction at LVNP is limited in C. purpureus, our data shows high within population variation among C. purpureus individuals, agreeing with other studies of $C$. purpureus population genetic structure (McDaniel \& Shaw, 2005, McDaniel et al., 2007, Skotnicki et al., 2004). These data indicate at the very least, the geothermal C. purpureus mosses are not one heat-adapted clone and at the most, they harbor distinct genetic variation between and among sites and it is correlated positively with Collembolan abundance, thus these data support the intriguing possibility that even at thermal extremes, microarthropods and mosses are engaged in mutualism.

This study could benefit from finer resolution of genetic analyses as well as from determining the likelihood of reproduction by testing the underlying the sex ratio structure of C. purpureus individuals (Charlesworth, 1984). In these sites the gametophytes appear without gametoecia, which could be a sign of temperature stress (Wahid et al., 2007) although underlying sex ratios could indicate that there is sufficient representation of both sexes. There is the possibility that if conditions were less extreme, sex 
could have been induced from stress relief. Sex after stress relief has been shown in Brassica species (Angadi et al., 2000), but more empirical tests on the effects of temporally alleviated stress on reproduction could yield great insight, and the moss system would be ideal for such laboratory based tests (Thornton et al., 2005). Further, in situ physiological measures of photosynthesis as well as biochemical assays assessing oxidative stress would help us to understand the physiological state of the mosses to help determine if the level of stress the plants are experiencing inhibit reproductive efforts (Zinn et al., 2010). Experimental cooling of cloned mosses could reveal whether sexual reproduction in stress tolerant mosses is facultative thus elucidating further insight into the constraints and plasticity of moss reproduction under thermal stress.

Moss spores have the potential to travel relatively long distances (Frahm, 2009, Hutsemekers et al., 2008), and it is possible that these $C$. purpureus populations originated from spores of more mesic environments, ended up in geothermal regions and developed heat tolerance, as $C$. purpureus can exhibit phenotypic plasticity and/or adaptation to stress (Jules \& Shaw, 1994, Robinson et al., 2000). Given that the surrounding area is rather remote, forested or geothermal, and lacking in C. purpureus (Eppley et al., 2011), it may be just as likely that sexual reproduction occurred at some point in history. 
This particular system deserves deeper investigation into why one hot site is more biologically dynamic than another hot site and if abiotic stress relief could change the reproductive biology in a thermally extreme system. Such explorations could inform previously undiscovered biotic and abiotic correlates that drive ecosystem-level dynamics.

At the opposite edge of the thermal spectrum mosses also thrive. In Antarctica, mosses are some of the most abundant of terrestrial life (Ochyra et al., 2008) and are at the forefront of life on the western Antarctic Peninsula (Ochyra, 1998, Robinson et al., 2003). It is here, in the coldest regions of Earth where global warming is most eminent that the mosses may finally be recognized as globally important ecosystem engineers (Lawton \& Jones, 1995, Turetsky et al., 2012). Mosses have been shown to buffer and insulate soils and in turn, with the help of the canopy soil fauna, speed up microbial decomposition and nutrient cycling (Turetsky et al., 2012), a trend that only increases with climate warming (Jassey et al., 2013, Robinson et al., 2003, Royles et al., 2013).

As described in Chapter 5, Simulated warming relieves barriers to sexual reproduction in mosses, we studied the effects of six years of simulated warming by Open Top Chambers (OTCs) on mosses and found that bryophyte and lichen cover increases with warming (Fig 5.4). These findings confirm studies of warming-induced increased moss biomass (Kennedy, 1995), but not other studies where moss cover decreased (Elmendorf et al., 2012, Wahren et 
al., 2005). We propose that by pointedly investigating representative mossplant functional groups in Antarctica we will be better suited to understand organismal to ecosystem-level processes (Diaz \& Cabido, 2001, Eviner \& Chapin III, 2003, Suding et al., 2008) and how these processes are effected by climate change (Cannone \& Pignatti, 2014, Chapin et al., 1996, Walther et $a l ., 2002)$.

Based on meta-analyses results, net mineralization and soil respiration rates are likely to increase, as are plant biomass and sexual reproduction with warming (Arft, 1999, Elmendorf et al., 2012, Jassey et al., 2013, Royles et al., 2013, Rustad et al., 2001). There are not enough data to broadly assess how divergent these findings would be if focused solely on the non-vascular plants. Further, bryophytes and lichens are often lumped into one response variable but they can occupy very different space and serve dissimilar functions in a community (Körner, 1994). More surveys and studies on the effects of warming on non-vascular plant species and communities are key, particularly in Antarctica, where the dominant vegetation is mosses and lichens, as our results suggest that some mosses are capable of benefitting from warming.

Based on reports of mixed plant communities providing evidence of increased sexual reproduction with warming (Arft, 1999, Day et al., 1999, Klady et al., 2011), we predicted that warming would similarly increase reproductive efforts in mosses. As part of our assessment on the effects of 
passive warming on cryptogam communities in western Antarctica, we tested how a common dioicous Antarctic moss species, $P$. alpinum differed from $P$. alpinum in control plots without warming. Our data support the hypothesis that warming would increase reproductive effort due to relaxed stress and sheds light on fundamental constraints on moss function and reproduction. Inhibition of sexual reproduction is predicted when a plant must primarily defend and protect itself in harsh landscapes (Herms \& Mattson, 1992, Martínez et al., 2004, Stark et al., 2000) as we have seen evidence of in both the thermal extremes of geothermal hot springs and Antarctic islands.

Up to this point I have discussed constraints on sexual reproduction in dioicous mosses as it relates to water and heat stress, and how interactions with microarthropods may help to alleviate sperm dispersal limitation. In Antarctic P. alpinum, we found that with alleviated cold stress via simulated warming, the mosses were less oxidatively stressed. P. alpinum in warmed plots displayed less antioxidant enzyme activity, higher protein content (Fig. 5.7), improved photosynthetic capacity (Fig. 5.6) and increased male and female sex organ expression compared to controls (Table 5.1). Although we did not see sporophytes in $P$. alpinum, we found more sporophytes of monoicous species in OTC plots than in control plots, indicating an overall increase in reproductive output by mosses with warming. These data support hypotheses that environmental extremes constrain sex (David et al., 2005, Eppley et al., 2011, Kis-Papo et al., 2003, Stark, 2002), and may be the 
primary reproductive limitation on dioicous species at extreme temperatures. With stress relief, reproductive barriers may shift towards sperm limitation.

If sexual reproduction increases in Antarctic mosses due to eminent warming, genetic variation on the landscape will also expand (Hamrick \& Godt, 1996). We can then predict that Antarctic vegetation will see unprecedented changes in the nearby future. Given the widespread occurrence of mosses in terrestrial maritime Antarctica, any increase in reproductive effort could dramatically change the genetic and physical landscape.

Based on the themes of this dissertation, an obvious path for further studies on the Antarctic moss system is not only through extended investigation into reproductive barriers, but also in the mosses relationship to the canopy biota. Microarthropod and microbial community structures in response to warming have been tested with results ranging from decreased diversity, to increased abundance, to fully differential effects based on species traits (Bokhorst et al., 2008, Makkonen et al., 2011, Nash et al., 2013, Royles et al., 2013). This is unsurprising based on the variety of responses to warming that microarthropod and microbial habitats (e.g. soil, moss, lichen, grass) have exhibited. In an effort to circumvent such plastic findings I would suggest conducting an in situ temporal assessment of Antarctic moss canopy VOC suites from male and female mosses with and without simulated warming. Along with these data, sex expression of mosses and physiological 
status should be collected. Further, quantifying relative abundances and diversity and activities of microarthropods and microbes living among the canopies would be a useful descriptive start. Upon establishing a biotic and metabolic baseline for Antarctic moss communities, we would be better equipped to conduct controlled and manipulative tests of the likely unprecedented effects of warming on moss ecology and reproductive biology in a moss-dominated landscape.

\section{Broad Conclusions}

The chapters of this dissertation reveal that the moss mating system is under-estimated and more complex than previously recognized. Taken together these data begin to unravel the many ways by which the mosses may have become as widespread and diverse as they are, given their modest aquatic origins. Mosses have pioneered their way onto every continent and will likely prevail as an important regulator of global biogeochemistry, and serve as early indicators of what is to come for terrestrial landscapes under

global climate change. I have discussed that mosses have sperm that may act as stress tolerant gametes on the landscape and that there is a scentmediated likely-mutualistic relationship between the ancient mosses and microarthropods that contributes significantly to our understanding of the evolution of terrestrial plant biology. I have tested the functional and interactive relationship of microarthropods and mosses in deliberate 
experiments as well as in the field where I focused on the extreme edges of life, showing that although extreme from an anthropocentric view, abiotic pressures do not necessarily limit the productivity of the complex bryosphere. I discuss how mosses are at the forefront of global climate change as they are affected by warming in ways not only crucial to their own biology, but to the future of terrestrial landscapes. Finally, I applied the fundamental scientific topic of multi-trophic interactions to engage middle school students in authentic research-based science education in Chapter 6, Mosscosms: a classroom activity to encourage ecosystem-level thinking. As a trained scientist it is key to learn how to disseminate the sometimes-complex information that we produce. By learning how to distill complex ecological concepts into education-appropriate material, I have furthered my understanding of science communication as well as the true scientific process.

This dissertation comprises an investigation into moss reproduction through the integration of multi-disciplinary scientific theory and practice that spans from genetics to organismal stress physiology, to multi-trophic ecology, yielding novel results that provide new ground by which to pursue the studies of reproductive success in mosses. 


\section{References}

Adams BJ, Bardgett RD, Ayres E et al. (2006) Diversity and distribution of Victoria Land biota. Soil Biology and Biochemistry, 38, 3003-3018.

Aerts R (2006) The freezer defrosting: global warming and litter decomposition rates in cold biomes. Journal of Ecology, 94, 713-724.

Alpert P (2000) The discovery, scope and puzzle of dessication tolerance in plants. Plant Ecology, 151, 5-17.

Anderson R (2002) Reforming Science Teaching: What Research Says About Inquiry. Journal of Science Teacher Education, 13, 1-12.

Andrew NR, Rodgerson L, Dunlop M (2003) Variation in invertebratebryophyte community structure at different spatial scales along altitudinal gradients. Journal of Biogeography, 30, 731-746.

Angadi S, Cutforth H, Miller P, Mcconkey B, Entz M, Brandt S, Volkmar K (2000) Response of three Brassica species to high temperature stress during reproductive growth. Canadian Journal of Plant Science, 80, 693-701.

Apel K, Hirt H (2004) Reactive oxygen species: metabolism, oxidative stress, and signal transduction. Annu. Rev. Plant Biol., 55, 373-399.

Applebee AN, Langer JA, Nystrand M, Gamoran A (2003) Discussion-based approaches to developing understanding: Classroom instruction and student performance in middle and high school English. American Educational Research Journal, 40, 685-730.

Appoloni S, Lekberg Y, Tercek MT, Zabinski CA, Redecker D (2008)

Molecular Community Analysis of Arbuscular Mycorrhizal Fungi in Roots of Geothermal Soils in Yellowstone National Park (USA). Microbial Ecology, 56, 649-659.

Arendt JD (1997) Adaptive intrinsic growth rates: An integration across taxa. Quarterly Review of Biology, 72, 149-177.

Arft AM, M. D. Walker, J. Gurevitch, J. M. Alatalo, M. S. Bret-Harte, M. Dale, M. Diemer, F. Gugerli, G. H. R. Henry, M. H. Jones, R. D. Hollister, I. S. Jónsdóttir, K. Laine, E. Lévesque, G. M. Marion, U. Molau, P. Mølgaard, U. Nordenhäll, V. Raszhivin, C. H. Robinson, G. Starr, A. Stenström, M. Stenström, Ø. Totland, P. L. Turner, L. J. Walker, P. J. Webber, J. M. Welker, and P. A. Wookey (1999) Responses of tundra plants to experimental warming: meta-analysis of the international tundra experiment. Ecological Monographs, 69, 491-511.

Asada K (1997) The role of ascorbate peroxidase and monodehydroascorbate reductase in H 2 O 2 scavenging in plants. Cold Spring Harbor Monograph Archive, 34, 715-735.

Ashman TL (2009) Sniffing out patterns of sexual dimorphism in floral scent. Functional Ecology, 23, 852-862. 
Ashman TL, Knight TM, Steets JA et al. (2004) Pollen limitation of plant reproduction: Ecological and evolutionary causes and consequences. Ecology, 85, 2408-2421.

Au DWT, Reunov AA, Wu RSS (1998) Four lines of spermatid development and dimorphic spermatozoa in the sea urchin Anthocidaris crassispina (Echinodermata, Echinoida). Zoomorphology, 118, 159-168.

Ayres E, Van Der Wal R, Sommerkorn M, Bardgett RD (2006) Direct uptake of soil nitrogen by mosses. Biology Letters, 2, 286-288.

Bakken S (1995) Effects of nitrogen supply and irradiance on growth and nitrogen status in the moss Dicranum majus from differently polluted areas. Journal Of Bryology, 18, 707-721.

Bardgett RD, Chan KF (1999) Experimental evidence that soil fauna enhance nutrient mineralization and plant nutrient uptake in montane grassland ecosystems. Soil Biology and Biochemistry, 31, 1007-1014.

Bargagli R, Broady PA, Walton DWH (1996) Preliminary investigation of the thermal biosystem of Mount Rittmann fumaroles (northern Victoria Land, Antarctica). Antarctic Science, 8, 121-126.

Barker DH, Stark LR, Zimpfer JF, Mcletchie ND, Smith SD (2005) Evidence of drought-induced stress on biotic crust moss in the Mojave Desert. Plant Cell and Environment, 28, 939-947.

Barrett SC, Harder LD, Worley AC (1996) The comparative biology of pollination and mating in flowering plants. Philosophical Transactions: Biological Sciences, 1271-1280.

Barrett SCH, And C. G. Eckert (1990) Variation and evolution of mating systems in seed plants. In: Biological approaches and evolutionary trends in plants. (ed Kawano S) pp 229-254. London, Academic Press.

Basnayake J, Ludlow MM, Cooper M, Henzell RG (1993) Genotypic varation of osmotic adjustment and desiccation tolerance in constrasting sorghum inbred lines. Field Crops Research, 35, 51-62.

Bawa K (1990) Plant-pollinator interactions in tropical rain forests. Annual review of ecology and systematics, 399-422.

Bazzaz FA, Chiariello NR, Coley PD, Pitelka LF (1987) Allocating resources to reproduction and defense. BioScience, 37, 58-67.

Bell G (1985) On the function of flowers. Proceedings of the Royal society of London. Series B. Biological sciences, 224, 223-265.

Bell RL, Smetana L, Binns I (2005) Simplifying inquiry instruction. The Science Teacher, 72, 30-33.

Bengtsson G, Hedlund K, Rundgren S (1991) Selective odor perception in the soil Collembola Onychiurus-armatus. Journal of Chemical Ecology, 17, 2113-2125.

Bernhard DL, Renzaglia KS (1995) Spermiogenesis in the moss Aulacomnium palustre. The Bryologist, 98, 52-70. 
Bewley JD (1973) Polyribosomes Conserved during Desiccation of the Moss Tortula ruralis Are Active. Plant Physiology, 51, 285-288.

Bewley JD (1979) Physiological Aspects of Desiccation Tolerance. Annual Review of Plant Physiology, 30, 195-238.

Bewley JD, Halmer P, Krochko JE, Winner WE (1978) Metabolism of a drought-tolerant and a drought-sensitive moss: respiration, ATP synthesis and carbohydrate status. In: Dry biological systems. (eds Crowe JH, Clegg JS). New York, USA, Academic Press.

Bewley JD, Tucker EB, Gwozdz EA (1974) The effects of stress on the metabolism of Tortula ruralis. In: Mechanisms of Regulation of Plant Growth, Bulletin 12. (eds Bieleski RL, Ferguson AR, Cresswell MM). Wellington, New Zealand, Royal Society New Zealand.

Beyer Jr WF, Fridovich I (1987) Assaying for superoxide dismutase activity: some large consequences of minor changes in conditions. Analytical biochemistry, 161, 559-566.

Bhowmick S, Zhu L, Mcginnis L, Lawitts J, Nath BD, Toner M, Biggers J (2003) Desiccation Tolerance of Spermatozoa Dried at Ambient Temperature: Production of Fetal Mice. Biology of Reproduction, 68, 1779-1786.

Bianchi G, Gamba A, Murelli C, Salamini F, Bartels D (1991) Novel carbohydrate metabolism in the resurrection plant Craterostigma plantagineum. Plant Journal, 1, 355-359.

Bilger W, Schreiber U, Bock M (1995) Determination of the quantum efficiency of photosystem II and of non-photochemical quenching of chlorophyll fluorescence in the field. Oecologia, 102, 425-432.

Bisang I, Ehrlen J, Hedenas L (2004) Mate limited reproductive success in two dioicous mosses. Oikos, 104, 291-298.

Bisang I, Ehrlen J, Hedenas L (2006) Reproductive effort and costs of reproduction do not explain female-biased sex ratios in the moss Pseudocalliergon trifarium (Amblystegiaceae). American Journal of Botany, 93, 1313-1319.

Bisang I, Hedenas L (2005) Sex ratio patterns in dioicous bryophytes revisited. Journal Of Bryology, 27, 207-219.

Blackman SA, Obendorf RL, Leopold AC (1992) Maturation Proteins and Sugars in Desiccation Tolerance of Developing Soybean Seeds. Plant Physiology, 100, 225-230.

Block W (1985) Arthropod interactions in an Antarctic terrestrial community. In: Antarctic nutrient cycles and food webs. pp 614-619. Springer.

Blumenfeld PC, Soloway E, Marx RW, Krajcik JS, Guzdial M, Palincsar A (1991) Motivating Project-Based Learning: Sustaining the Doing, Supporting the Learning. Educational Psychologist, 26, 369-398. 
Bokhorst S, Huiskes A, Convey P, Aerts R (2007) The effect of environmental change on vascular plant and cryptogam communities from the Falkland Islands and the Maritime Antarctic. BMC ecology, 7, 15.

Bokhorst S, Huiskes A, Convey P, Sinclair BJ, Lebouvier M, Van De Vijver B, Wall DH (2011) Microclimate impacts of passive warming methods in Antarctica: implications for climate change studies. Polar Biology, 34, 1421-1435.

Bokhorst S, Huiskes A, Convey P, Van Bodegom PM, Aerts R (2008) Climate change effects on soil arthropod communities from the Falkland Islands and the Maritime Antarctic. Soil Biology and Biochemistry, 40, 1547-1556.

Bolter M, Blume HP, Schneider D, Beyer L (1997) Soil properties and distributions of invertebrates and bacteria from King George Island (Arctowski Station), maritime Antarctic. Polar Biology, 18, 295-304.

Bonini I, Casini F, Chiarucci A, De Dominicis V (2005) The bryophyte flora of the geothermal field of Sasso Pisano (Pisa, Italy). Crytogamie, Bryologie, 26, 291-299.

Borcard D, Legendre P (1994) Environmental control and spatial structure in ecological communities: an example using oribatid mites (Acari, Oribatei). Environmental and Ecological Statistics, 1, 37-61.

Bracegirdle TJ, Connolley WM, Turner J (2008) Antarctic climate change over the twenty first century. Journal of Geophysical Research: Atmospheres, 113, D03103.

Bradford MM (1976) A rapid and sensitive method for the quantitation of microgram quantities of protein utilizing the principle of protein-dye binding. Analytical biochemistry, 72, 248-254.

Bramley-Alves J, King DH, Robinson SA, Miller RE (2014) Dominating the Antarctic Environment: bryophytes in a time of change. In: Photosynthesis in Bryophytes and Early Land Plants. pp 309-324. Springer.

Branch G (2013) Defending Science Education: Climate as a Second Front for Biologists. BioScience, 63, 717-718.

Brewbaker JL, Kwack BH (1963) The essential role of calcium ion in pollen germination and pollen tube growth. American Journal of Botany, 859865.

Brickhouse NW (1990) Teachers' beliefs about the nature of science and their relationship to classroom practice. Journal of teacher education, 41, 5362.

Bromwich DH, Nicolas JP, Monaghan AJ, Lazzara MA, Keller LM, Weidner GA, Wilson AB (2014) Corrigendum: Central West Antarctica among the most rapidly warming regions on Earth. Nature Geoscience, 7, 7676 . 
Brownell S, Wenderoth MP, Theobald R et al. (2014) How Students Think about Experimental Design: Novel Conceptions Revealed by in-Class Activities. BioScience, 64, 125-137.

Brownell SE, Kloser MJ, Fukami T, Shavelson R (2012) Undergraduate biology lab courses: comparing the impact of traditionally-based "cookbook" and authentic research-based courses on student lab experiences. Journal of College Science Teaching.

Bruno JF, Stachowicz JJ, Bertness MD (2003) Inclusion of facilitation into ecological theory. Trends in Ecology \& Evolution, 18, 119-125.

Buckland-Nicks J (1998) Prosobranch parasperm: Sterile germ cells that promote paternity? Micron, 29, 267-280.

Buckland-Nicks J, Bryson I, Hart L, Partridge V (1999) Sex and a snail's sperm: on the transport, storage and fate of dimorphic sperm in Littorinidae. Invertebrate Reproduction \& Development, 36, 145-152.

Burd M (1994) Bateman's Principle and plant reproduction: the role of pollen limitation in fruit and seed set. Botanical Review, 60, 83-139.

Cannone N, Pignatti S (2014) Ecological responses of plant species and communities to climate warming: upward shift or range filling processes? Climatic Change, 1-14.

Casanova-Katny MA, Cavieres LA (2012) Antarctic moss carpets facilitate growth of Deschampsia antarctica but not its survival. Polar Biology, 35, 1869-1878.

Chapin FS, Bret-Harte MS, Hobbie SE, Zhong H (1996) Plant functional types as predictors of transient responses of arctic vegetation to global change. Journal of Vegetation Science, 7, 347-358.

Chapin FSI, Autumn K, Pugnaire FI (1993) Evolution of suites of traits in response to environmental stress. American Naturalist, 142, S78-S92.

Charlesworth B, Charlesworth D (1999) The genetic basis of inbreeding depression. Genetical Research, 74, 329-340.

Charlesworth D (1984) Androdioecy and the evolution of dioecy. Biological Journal of the Linnean Society, 22, 333-348.

Chen G-X, Asada K (1989) Ascorbate peroxidase in tea leaves: occurrence of two isozymes and the differences in their enzymatic and molecular properties. Plant and Cell Physiology, 30, 987-998.

Chiarucci A, Calderisi M, Casini F, Bonini I (2008) Vegetation at the Limits for Vegetation: Vascular Plants, Bryophytes and Lichens in a Geothermal Field. In: Folia Geobotanica. pp 19-33, Springer Science \& Business Media B.V.

Chippindale AK, Gibbs AG, Sheik M, Yee KJ, Djawdan M, Bradley TJ, Rose MR (1998) Resource acquisition and the evolution of stress resistance in Drosophila melanogaster. Evolution, 52, 1342-1352. 
Chisholm C, Lindo Z, Gonzalez A (2011) Metacommunity diversity depends on connectivity and patch arrangement in heterogeneous habitat networks. Ecography.

Churchland C, Weatherall A, Briones MJ, Grayston SJ (2012) Stable,Äêisotope labeling and probing of recent photosynthates into respired $\mathrm{CO} 2$, soil microbes and soil mesofauna using a xylem and phloem stem, Âêinjection technique on Sitka spruce (Picea sitchensis). Rapid Communications in Mass Spectrometry, 26, 2493-2501.

Clarke LJ, Ayre DJ, Robinson SA (2008) Somatic mutation and the Antarctic ozone hole. Journal of Ecology, 96, 378-385.

Clarke LJ, Ayre DJ, Robinson SA (2009) Genetic structure of East Antarctic populations of the moss Ceratodon purpureus. Antarctic Science, 21, 51-58.

Clayton-Greene KA, Green TGA, Staples B (1977) Studies of Dawsonia superba. 1. Antherozoid Dispersal. The Bryologist, 80, 439-444.

Convey P (1994) Modelling reproductive effort in sub-and maritime Antarctic mosses. Oecologia, 100, 45-53.

Convey P (1996) Overwintering strategies of terrestrial invertebrates in Antarctica-the significance of flexibility in extremely seasonal environments. European Journal of Entomology, 93, 489-506.

Convey P, Lewis Smith R, Hodgson D, Peat H (2000) The flora of the South Sandwich Islands, with particular reference to the influence of geothermal heating. Journal of Biogeography, 27, 1279-1295.

Convey P, Lewis Smith RI (2006) Geothermal bryophyte habitats in the South Sandwich Islands, maritime Antarctic. Journal of Vegetation Science, 17, 529-538.

Convey P, Pugh PJA, Jackson C, Murray AW, Ruhland CT, Xiong FS, Day TA (2002) Response of Antarctic terrestrial microarthopods to longterm climate manipulaitons. Ecology, 83, 3130-3140.

Convey P, Smith RIL (1993) Investment in sexual reproduction by Antarctic mosses. Oikos, 293-302.

Cook MP (2006) Visual representations in science education: The influence of prior knowledge and cognitive load theory on instructional design principles. Science Education, 90, 1073-1091.

Cornelissen J (1996) An experimental comparison of leaf decomposition rates in a wide range of temperate plant species and types. Journal of Ecology, 573-582.

Cove D, Bezanilla M, Harries P, Quatrano R (2006) Mosses as model systems for the study of metabolism and development. Annual Review of Plant Biology, 57, 497-520.

Coxson DS, Mcintyre DD, Vogel HJ (1992) Pulse Release of Sugars and Polyols from Canopy Bryophytes in Tropical Montane Rain Forest (Guadeloupe, French West Indies). Biotropica, 24, 121-133. 
Crepet WL (1984) Advanced (constant) insect pollination mechanims-pattern of evoluton and implicaiotns vis-a-vis angiosperm diversity Annals of the Missouri Botanical Garden, 71, 607-630.

Cronberg N (2012) Animal-mediated fertilization in bryophytes: a parallel or precursor to insect pollination in angiosperms? Lindbergia, 35, 76-85.

Cronberg N, Natcheva R, Hedlund K (2006) Microarthropods mediate sperm transfer in mosses. Science, 313, 1255-1255.

Crosby MR, Garden MB (2000) A Checklist of the Mosses, Missouri Botanical Garden St. Louis.

Crowe JH, Crowe LM, Chapman D (1984) Preservation of membranes in anhydrobiotic organisms-the role of trehalose. Science, 223, 701-703.

Crowe JH, Hoekstra FA, Crowe LM (1992) Anhydrobiosis. Annual Review of Physiology, 54, 579-599.

Croxall JP, Trathan PN, Murphy EJ (2002) Environmental Change and Antarctic Seabird Populations. Science, 297, 1510-1514.

Crum HA (1973) Mosses of the Great Lakes Forest, Michigan, Ann Arbor, University of Michigan.

Cuming AC, Cho SH, Kamisugi Y, Graham H, Quatrano RS (2007)

Microarray analysis of transcriptional responses to abscisic acid and osmotic, salt, and drought stress in the moss, Physcomitrella patens. New Phytologist, 176, 275-287.

Curtis C (1971) Disc electrophoretic comparisons of proteins and peroxidases from Phaseolus vulgaris leaves infected with Agrobacterium tumefaciens. Canadian Journal of Botany, 49, 333-337.

Dahlman L, Zetherström M, Sundberg B, Näsholm T, Palmqvist K (2002) Measuring ergosterol and chitin in lichens. In: Protocols in Lichenology. pp 348-362. Springer.

Davey A, Marchant HJ (1983) Seasonal variation in nitrogen fixation by Nostoc commune Vaucher at the Vestfold Hills, Antarctica. Phycologia, 22, 377-385.

Davey ML, Currah RS (2006) Interactions between mosses (Bryophyta) and fungi. Botany, 84, 1509-1519.

Davey ML, Heegaard E, Halvorsen R, Ohlson M, Kauserud HV (2012) Seasonal trends in the biomass and structure of bryophyte-associated fungal communities explored by 454 pyrosequencing. New Phytologist, 195, 844-856.

David JR, Araripe LO, Chakir M et al. (2005) Male sterility at extreme temperatures: a significant but neglected phenomenon for understanding Drosophia climatic adaptations. Journal of Evolutionary Biology 18, 838-846.

Davidson A, Harborne J, Longton R (1990) The acceptability of mosses as food for generalist herbivores, slugs in the Arionidae. Botanical Journal of the Linnean Society, 104, 99-113. 
Davis R (1981) Structure and function of two Antarctic terrestrial moss communities. Ecological Monographs, 51, 125-143.

Day T, Ruhland C, Grobe C, Xiong F (1999) Growth and reproduction of Antarctic vascular plants in response to warming and UV radiation reductions in the field. Oecologia, 119, 24-35.

Day TA, Ruhland CT, Xiong FS (2008) Warming increases aboveground plant biomass and $\mathrm{C}$ stocks in vascular-plant-dominated Antarctic tundra. Global Change Biology, 14, 1827-1843.

Deboer GE (2000) Scientific literacy: Another look at its historical and contemporary meanings and its relationship to science education reform. Journal of Research in Science Teaching, 37, 582-601.

Deluca TH, Zackrisson O, Nilsson M-C, Sellstedt A (2002) Quantifying nitrogen-fixation in feather moss carpets of boreal forests. Nature, 419, 917-920.

Díaz S, Cabido M (2001) Vive la difference: plant functional diversity matters to ecosystem processes. Trends in Ecology \& Evolution, 16, 646-655.

Dilks TJK, Proctor MCF (1979) Photosynthesis, respiration and water content in bryophytes. New Phytologist, 82, 97-114.

Ding Q, Steig EJ (2013) Temperature Change on the Antarctic Peninsula Linked to the Tropical Pacific*. Journal of Climate, 26.

Doebeli M, Hauert C (2005) Models of cooperation based on the Prisoner's Dilemma and the Snowdrift game. Ecology letters, 8, 748-766.

Downie R, Convey P, Mcinnes S, Pugh P (2000) The non-marine invertebrate fauna of Deception Island (Maritime Antarctic): a baseline for a comprehensive biodiversity database. Polar Record, 36, 297-304.

Ducklow HW, Baker K, Martinson DG et al. (2007) Marine pelagic ecosystems: the West Antarctic Peninsula. Philosophical Transactions of the Royal Society B: Biological Sciences, 362, 67-94.

Dudareva N, Pichersky E (2010) Biology of floral scent, CRC Press.

During HJ (1979) Life strategies of bryophytes: a preliminary review. Lindbergia, 2-18.

Eckstein RL, Karlsson PS (1999) Recycling of nitrogen among segments of Hylocomium splendens as compared with Polytrichum commune: implications for clonal integration in an ectohydric bryophyte. Oikos, 87-96.

Ederli L, Reale L, Ferranti F, Pasqualini S (2004) Responses induced by high concentration of cadmium in Phragmites australis roots. Physiologia Plantarum, 121, 66-74.

Ehrlén J, Bisang I, Hedenäs L (2000) Costs of sporophyte production in the moss, Dicranum polysetum. Plant Ecology, 149, 207-217.

Eilam B (2012) System thinking and feeding relations: learning with a live ecosystem model. Instructional Science, 40, 213-239. 
Elmarsdottir A, Ingimarsdottir M, Hansen I, Olafsson JS, Olafsson E (2003) Vegetation and invertebrates in three geothermal areas in Iceland. In: International Geothermal Conference. (ed Iceland GaO) pp 49-55, Reykjavik, Iceland.

Elmendorf SC, Henry GH, Hollister RD et al. (2012) Global assessment of experimental climate warming on tundra vegetation: heterogeneity over space and time. Ecology letters, 15, 164-175.

Eppley SM, Rosenstiel TN, Graves CB, Garcia E (2011) Limits to sexual reproduction in geothermal bryophytes. International Journal of Plant Sciences, 172, 870-978.

Eppley SM, Taylor PT, Jesson LK (2007) Self-fertilization in mosses: a comparison of heterozygote deficiency between species with combined versus separate sexes. Heredity, 98, 38-44.

Equihua M, Usher MB (1993) Impact of Carpets of the Invasive Moss Campylopus Introflexus on Calluna Vulgaris Regeneration. Journal of Ecology, 81, 359-365.

Etourneau JC, L. G.; Willmott, V.; Kim, J.-H.; Barbara, L.; Leventer, A.; Schouten, S.; Damsté, J. S. Sinninghe; Bianchini, A.; Klein, V.; Crosta, X.; Massé, G (2013) Holocene climate variations in the western Antarctic Peninsula: evidence for sea ice extent predominantly controlled by insolation and ENSO variability changes. Climate of the Past Discussions, 9.

Evans JR (1989) Photosynthesis and nitrogen relationships in leaves of C3 plants. Oecologia, 78, 9-19.

Eviner VT, Chapin Iii FS (2003) Functional matrix: A Conceptual Framework for Predicting Multiple Plant Effects on Ecosystem Processes. Annual Review of Ecology, Evolution, and Systematics, 34, 455-485.

Excoffier L, Smouse PE, Quattro JM (1992) Analysis of molecular variance inferred from metric distances among DNA haplotypes: application to human mitochondrial DNA restriction data. Genetics, 131, 479-491.

Fenster CB, Armbruster WS, Wilson P, Dudash MR, Thomson JD (2004) Pollination syndromes and floral specialization. Annual Review of Ecology Evolution and Systematics, 35, 375-403.

Forcada J, Trathan PN, Reid K, Murphy EJ, Croxall JP (2006) Contrasting population changes in sympatric penguin species in association with climate warming. Global Change Biology, 12, 411-423.

Fountain MT, Hopkin SP (2005) Folsomia candida (Collembola): A "Standard" Soil Arthropod. Annu. Rev. Entomol., 50, 201-222.

Frahm J-P (1980) Synopsis of the Genus Campylopus in North America North of Mexico. The Bryologist, 83, 570-588.

Frahm J-P (2009) Diversity, dispersal and biogeography of bryophytes (mosses). In: Protist Diversity and Geographical Distribution. pp 43-50. Springer. 
Fraser CI, Terauds A, Smellie J, Convey P, Chown SL (2014) Geothermal activity helps life survive glacial cycles. Proceedings of the National Academy of Sciences.

Fraser WR, Trivelpiece WZ, Ainley DG, Trivelpiece SG (1992) Increases in Antarctic penguin populations - reduced competition with whales, or a loss of sea ice due to environmental warming? Polar Biology, 11, 525531.

Freestone AL (2006) Facilitation drives local abundance and regional distribution of a rate plant in a harsh environment. Ecology, 87, 27282735.

Ganzert L, Lipski A, Hubberten H-W, Wagner D (2011) The impact of different soil parameters on the community structure of dominant bacteria from nine different soils located on Livingston Island, South Shetland Archipelago, Antarctica. FEMS Microbiology Ecology, 76, 476-491.

Garbary DJ, Renzaglia KS, Duckett JG (1993) The phylogeny of land plants: a cladistic analysis based on male gametogenesis. Plant Systematics and Evolution, 188, 237-269.

Garg AK, Kim JK, Owens TG, Ranwala AP, Do Choi Y, Kochian LV, Wu RJ (2002) Trehalose accumulation in rice plants confers high tolerance levels to different abiotic stresses. Proceedings of the National Academy of Sciences of the United States of America, 99, 15898-15903. Gerson U (1969) Moss-Arthropod Associations. The Bryologist, 72, 495-500. Giard A (1894) L'anhydrobiose en ralentissement des phénomènes vitaux. Comptes rendus des séances de la Société de biologie et de ses filiales, 46, 497.

Gibson RJH, Miller-Brown D (1927) Fertilization of bryophyta . Polytrichum commune. (Preliminary note.). Annals of Botany, os-41, 190-191.

Gill SS, Tuteja N (2010) Reactive oxygen species and antioxidant machinery in abiotic stress tolerance in crop plants. Plant Physiology and Biochemistry, 48, 909-930.

Ginzburg LR, Jensen CX (2004) Rules of thumb for judging ecological theories. Trends in Ecology \& Evolution, 19, 121-126.

Gitelson A, Buschmann C, Lichtenthaler H (1999) The chlorophyll fluorescence ratio F735/F700 as an accurate measure of the chlorophyll content in plants. Remote sensing of environment, 69, 296-302.

Glazer I, Gaugler R, Segal D (1991) Genetics of the nematode Heterorhabditis bacteriophora strain HP88- the diversity of beneficial traits. Journal of Nematology, 23, 324-333.

Glime JM (2007) Bryophyte Ecology. Ebook sponsored by Michigan Technical University and the International Association of Bryologists.

Glime JM, Hong WS (1997) Relationships of geothermal bryophyte communities to soil characteristics at Thermal Meadow, Hotsprings 
Island, Queen Charlotte Islands, Canada. Journal Of Bryology, 19, 435-448.

Goffinet B, Shaw AJ (2000, 2009) Bryophyte Biology, Cambridge University Press Cambridge. First and Second Editions.

Gonzalez A, Lawton J, Gilbert F, Blackburn T, Evans-Freke I (1998)

Metapopulation dynamics, abundance, and distribution in a microecosystem. Science, 281, 2045-2047.

Gornall J, Is J, Woodin S, Van Der Wal R (2007) Arctic mosses govern belowground environment and ecosystem processes. Oecologia, 153, 931-941.

Gou XP, Yuan T, Wei XP, Russell SD (2009) Gene expression in the dimorphic sperm cells of Plumbago zeylanica: transcript profiling, diversity, and relationship to cell type. Plant Journal, 60, 33-47.

Gradstein SR, Sipman HJM (1978) Taxonomy and world distribution of Campylopus introflexus and C. pilifer (= C.polytrichoides): a new synthesis. Bryologist, 81, 114-121.

Grassle JF (1986) The ecology of deep-sea hydrothermal vent communities. Advances in Marine Biology, 23, 301-362.

Grime JP (1977) Evidence for existence of 3 primary strategies in pants and is relevance to ecological and evolutionary theory. American Naturalist, 111, 1169-1194.

Grishkan I, Korol AB, Nevo E, Wasser SP (2002) Ecological stress and sex evolution in soil microfungi. Proceedings of the Royal Society of London Series B-Biological Sciences, 270, 13-18.

Grout AJ (ed) (1933) Moss flora of North America, North of Mexico, Newfane, Vermont, Grout.

Haag RW (1974) Nutrient limitations to plant production in two tundra communities. Canadian Journal of Botany, 52, 103-116.

Hamrick JL, Godt MJW (1996) Effects of Life History Traits on Genetic Diversity in Plant Species. Philosophical Transactions of the Royal Society of London. Series B: Biological Sciences, 351, 1291-1298.

Hanauer DI, Frederick J, Fotinakes B, Strobel SA (2012) Linguistic Analysis of Project Ownership for Undergraduate Research Experiences. $C B E$ Life Sciences Education, 11, 378-385.

Handelsman J, Ebert-May D, Beichner R et al. (2004) Scientific teaching. Science, 304, 521-522.

Hayakawa Y (2007) Parasperm: morphological and functional studies on nonfertile sperm. Ichthyological Research, 54, 111-130.

Hayakawa Y, Kobayashi M, Munehara H, Watanabe A, Onitake K (2007) Spermatogenesis involving parasperm production in the marine cottoid fish, Hemilepidotus gilberti. Raffles Bulletin of Zoology, 29-35.

Heiri O, Lotter AF, Lemcke G (2001) Loss on ignition as a method for estimating organic and carbonate content in sediments: reproducibility and comparability of results. Journal of paleolimnology, 25, 101-110. 
Hemborg AM, Bond WJ (2005) Different rewards in female and male flowers can explain the evolution of sexual dimorphism in plants. Biological Journal of the Linnean Society, 85, 97-109.

Herms DA, Mattson WJ (1992) The dilemma of plants: to grow or defend. Quarterly Review of Biology, 283-335.

Higginson DM, Pitnick S (2011) Evolution of intra-ejaculate sperm interactions: do sperm cooperate? Biological Reviews, 86, 249-270.

Hirano T, Hoshino Y (2010) Sperm dimorphism in terms of nuclear shape and microtubule accumulation in Cyrtanthus mackenii. Sexual Plant Reproduction, 23, 153-162.

Hochachka PW, Somero GN (2002) Biochemical adaptation: mechanism and process in physiological evolution, Oxford University Press, USA.

Hodgson AN (1997) Paraspermatogenesis in gastropod molluscs. Invertebrate Reproduction \& Development, 31, 31-38.

Hodson D (1996) Laboratory work as scientific method: Three decades of confusion and distortion. Journal of Curriculum studies, 28, 115-135.

Hoekstra FA, Golovina EA, Buitink J (2001) Mechanisms of plant desiccation tolerance. Trends in plant science, 6, 431-438.

Hoekstra FA, Vanroekel T (1988) Desiccation tolerance of Papaver-dubium pollen during its development in the anther-possible role of phospholipid-composition and sucrose content. Plant Physiology, 88, 626-632.

Hoffmann AA, Harshman LG (1999) Desiccation and starvation resistance in Drosophila: patterns of variation at the species, population and intrapopulation levels. Heredity, 83, 637-643.

Hoffmann AA, Parsons PA (1989) An integrated approach to environmentalstress tolerance and life-history variation-desiccation tolerance in Drosophila. Biological Journal of the Linnean Society, 37, 117-136.

Hopkin SP (1997) Biology of the springtails (Insecta: Collembola), Oxford University Press, USA.

Horsley K, Stark LR, Mcletchie DN (2011) Does the silver moss Bryum argenteum exhibit sex-specific patterns in vegetative growth rate, asexual fitness or prezygotic reproductive investment? Annals of Botany, 107, 897-907.

Housman D, Powers H, Collins A, Belnap J (2006) Carbon and nitrogen fixation differ between successional stages of biological soil crusts in the Colorado Plateau and Chihuahuan Desert. Journal of Arid Environments, 66, 620-634.

Hughes AR, Inouye BD, Johnson MTJ, Underwood N, Vellend M (2008) Ecological consequences of genetic diversity. Ecology letters, 11, 609623. 
Hutsemekers V, Dopagne C, Vanderpoorten A (2008) How far and how fast do bryophytes travel at the landscape scale? Diversity and Distributions, 14, 483-492.

Iba K (2002) Acclimative response to temperature stress in higher plants: approaches of gene engineering for temperature tolerance. Annual Review of Plant Biology, 53, 225-245.

Ingram J, Bartels D (1996) The molecular basis of dehydration tolerance in plants. Annual Review of Plant Physiology and Plant Molecular Biology, 47, 377-403.

Island IB, Arctic C (2001) Embryophytes on land: the Ordovician to Lochkovian (Lower Devonian) record. Plants invade the land: evolutionary and environmental perspectives, 3.

Jamieson B, Rouse G (1989) The spermatozoa of the Polychaeta (Annelida): an ultrastructural review. Biological Reviews, 64, 93-157.

Jassey VE, Chiapusio G, Binet P et al. (2013) Above and belowground linkages in Sphagnum peatland: climate warming affects plantmicrobial interactions. Global Change Biology, 19, 811-823.

Jauhiainen J, Wallén B, Malmer N (1998) Potential NH4+ and NO3- uptake in seven Sphagnum species. New Phytologist, 138, 287-293.

Jennings T, Barkham J (1975) Food of slugs in mixed deciduous woodland. Oikos, 211-221.

Jesson LK, Taylor P, Eppley SM (2007) Inbreeding depression in mosses occurs in a species with separate sexes but not in a species with combined sexes. American Journal of Botany, (in press).

Johnson DL, Wellington W (1980) Predation of Apochthonius minimus (Pseudoscorpionida: Chthoniidae) on Folsomia Candida (Collembola: Isotomidae) II. Effects of predation on prey populations. Researches on Population Ecology, 22, 353-365.

Johnson MT, Stinchcombe JR (2007) An emerging synthesis between community ecology and evolutionary biology. Trends in Ecology \& Evolution, 22, 250-257.

Johnson SD, Steiner KE (2000) Generalization versus specialization in plant pollination systems. Trends in Ecology \& Evolution, 15, 140-143.

Jolliffe I (2005) Principal component analysis, Wiley Online Library. Jost L (2006) Entropy and diversity. Oikos, 113, 363-375.

Jules ES, Shaw AJ (1994) Adaptation to metal-contaminated soils in populations of the moss, Ceratodon purpureus: vegetative growth and reproductive expression. American Journal of Botany, 791-797.

Jurenka R, Terblanche JS, Klok CJ, Chown SL, Krafsur ES (2007) Cuticular lipid mass and desiccation rates in Glossina pallidipes: interpopulation variation. Physiological Entomology, 32, 287-293. 
Kachalkin A, Glushakova A, Yurkov A, Chernov IY (2008) Characterization of yeast groupings in the phyllosphere of Sphagnum mosses. Microbiology, 77, 474-481.

Kaiser K, Outlaw WH, Ziegler H (1985) Sucrose content of receptive archegonia of the moss Bryum capillare; Hedw. Naturwissenschaften, 72, 378-379.

Kappen L (1993) Plant activity under snow and ice, with particular reference to lichens. Arctic, 46, 297-302.

Kappen L, Smith CW (1980) Heat tolerance of tow Cladonia species and Campylous praemorsus in a hot steam vent area of Hawaii. Oecologia, 47, 184-189.

Kashyap A, Pandey K, Gupta R (1991) Nitrogenase activity of the antarctic cyanobacteriumNostoc commune: Influence of temperature. Folia Microbiologica, 36, 557-560.

Kennedy AD (1995) Simulated climate change: are passive greenhouses a valid microcosm for testing the biological effects of environmental perturbations? Global Change Biology, 1, 29-42.

Kennedy M (1994) The Ownership Project: An Experiment in Student Equity. Social Studies Review, 33, 24-30.

Kenrick P, Crane PR (1997) The origin and early evolution of land plants. Nature, 389, 33-39.

Keuper F, Dorrepaal E, Van Bodegom PM, Aerts R, Van Logtestijn RS, Callaghan TV, Cornelissen JH (2011) A Race for Space? How Sphagnum fuscum stabilizes vegetation composition during long-term climate manipulations. Global Change Biology, 17, 2162-2171.

Keys CW, Bryan LA (2001) Co-constructing inquiry-based science with teachers: Essential research for lasting reform. Journal of Research in Science Teaching, 38, 631-645.

King DA (2004) Climate change science: adapt, mitigate, or ignore? Science(Washington), 303, 176-177.

Kip N, Van Winden JF, Pan Y et al. (2010) Global prevalence of methane oxidation by symbiotic bacteria in peat-moss ecosystems. Nature Geoscience, 3, 617-621.

Kis-Papo T, Kirzhner V, Wasser SP, Nevo E (2003) Evolution of genomic diversity and sex at extreme environments: Fungal life under hypersaline Dead Sea Stress. Proceedings of the National Academy of Sciences of the United States of America, 100, 14970-14975.

Klady RA, Henry GHR, Lemay V (2011) Changes in high arctic tundra plant reproduction in response to long-term experimental warming. Global Change Biology, 17, 1611-1624.

Klanderud K (2008) Species-specific responses of an alpine plant community under simulated environmental change. Journal of Vegetation Science, 19, 363-372. 
Klatt CG, Wood JM, Rusch DB et al. (2011) Community ecology of hot spring cyanobacterial mats: predominant populations and their functional potential. The ISME journal, 5, 1262-1278.

Klironomos JN, Kendrick B (1995) Relationships among microarthropods, fungi, and their environment. In: The significance and regulation of soil biodiversity. pp 209-223. Springer.

Klironomos JN, Widden P, Deslandes I (1992) Feeding preferences of the collembolan Folsomia candida in relation to microfungal successions on decaying litter. Soil Biology and Biochemistry, 24, 685-692.

Kloser MJ, Brownell SE, Shavelson RJ, Fukami T (2013) Effects of a Research-Based Ecology Lab Course: A Study of Nonvolunteer Achievement, Self-Confidence, and Perception of Lab Course Purpose. Journal of College Science Teaching, 42.

Knight TM, Steets JA, Vamosi JC et al. (2005) Pollen limitation of plant reproduction: pattern and process. Annual Review of Ecology, Evolution, and Systematics, 467-497.

Knudsen JT, Gershenzon J (2006) The chemical diversity of floral scent. Biology of floral scent, 27-52.

Knudsen JT, Tollsten L, Bergstrom LG (1993) Floral scents - a checklist of volatile compounds isolated by headspace techniques. Phytochemistry, 33, 253-280.

Koranda M, Kerschbaum S, Wanek W, Zechmeister H, Richter A (2007) Physiological responses of bryophytes Thuidium tamariscinum and Hylocomium splendens to increased nitrogen deposition. Annals of Botany, 99, 161-169.

Körner C (1994) Scaling from species to vegetation: the usefulness of functional groups. In: Biodiversity and ecosystem function. pp 117-140. Springer.

Koster KL (1991) Glass-formation and desiccation tolerance in seeds. Plant Physiology, 96, 302-304.

Koster KL, Leopold AC (1988) Sugars and desiccation tolerance in seeds. Plant Physiology, 88, 829-832.

Krab EJ, Cornelissen JHC, Lang SI, Van Logtestijn RSP (2008) Amino acid uptake among wide-ranging moss species may contribute to their strong position in higher-latitude ecosystems. Plant and Soil, 304, 199208.

Lawton E (1971) Moss Flora of the Pacific Northwest, Tokyo, Japan, Hattori Botanical Laboratory.

Lawton JH, Jones CG (1995) Linking species and ecosystems: organisms as ecosystem engineers. In: Linking species \& ecosystems. pp 141-150. Springer.

Lee PE, Wilkes A (1965) Polymorphic spermatozoa in the hymenopterous wasp Dahlbominus. Science, 147, 1445-\&. 
Leibold MA, Holyoak M, Mouquet N et al. (2004) The metacommunity concept: a framework for multi-scale community ecology. Ecology letters, 7, 601-613.

Levin SA (1992) The problem of pattern and scale in ecology. Ecology, 73, 1943-1967.

Levitan DR (2000) Sperm velocity and longevity trade off each other and influence fertilization in the sea urchin Lytechinus variegatus. Proceedings of the Royal Society of London Series B-Biological Sciences, 267, 531-534.

Levitan DR, Petersen C (1995) Sperm limitation in the sea. Trends in Ecology \& Evolution, 10, 228-231.

Ligrone R, Duckett JG, Renzaglia KS (2012) Major transitions in the evolution of early land plants: a bryological perspective. Annals of Botany, 109, 851-871.

Lindo Z, Gonzalez A (2010) The bryosphere: an integral and influential component of the Earth's biosphere. Ecosystems, 13, 612-627.

Lindo Z, Winchester NN (2006) A comparison of microarthropod assemblages with emphasis on oribatid mites in canopy suspended soils and forest floors associated with ancient western redcedar trees. Pedobiologia, 50, 31-41.

Lisowski M, Disinger JF (1991) The effect of field-based instruction on student understandings of ecological concepts. The Journal of Environmental Education, 23, 19-23.

Liu J, Curry JA, Martinson DG (2004) Interpretation of recent Antarctic sea ice variability. Geophysical Research Letters, 31, L02205.

Lively CA, Lyons EJ, Peters AD, Jokela J (1998) Environmental stress and the maintenance of sex in a freshwater snail. Evolution, 52, 1482-1486.

Longton RE (1976) Reproductive biology and evolutionary potential in bryophytes. Journal of the Hattori Botanical Laboratory, 41, 205-223.

Longton RE (1988) Biology of polar bryophytes and lichens, CUP Archive.

Longton RE, Greene SW (1979) Experimental studies of growth and reproduction in the moss Pleurozium shreberi (Brid.) Mitt. Journal of Bryology, 10, 321-338.

Lord EM, Russell SD (2002) The mechanisms of pollination and fertilization in plants. Annual Review of Cell and Developmental Biology, 18, 81105.

Loreau M, Naeem S, Inchausti P et al. (2001) Ecology - Biodiversity and ecosystem functioning: Current knowledge and future challenges. Science, 294, 804-808.

Lovelock CE, Jackson AE, Melick DR, Seppelt RD (1995) Reversible Photoinhibition in Antarctic Moss during Freezing and Thawing. Plant Physiology, 109, 955-961. 
Lu J-C, Chen F, Xu H-R, Huang Y-F, Lu N-Q (2007) Comparison of Three Sperm-Counting Methods for the Determination of Sperm Concentration in Human Semen and Sperm Suspensions. Lab Med, 38, 232-236.

Lutzen J, Jespersen A, Takahashi T, Kai T (2004) Morphology, structure of dimorphic sperm, and reproduction in the hermaphroditic commensal bivalve Pseudopythina tsurumaru (Galeommatoidea : Kellidae). Journal of Morphology, 262, 407-420.

Mahmoud AMA, Depoorter B, Piens N, Comhaire FH (1997) The performance of 10 different methods for the estimation of sperm concentration. Fertility and Sterility, 68, 340-345.

Makkonen M, Berg MP, Van Hal JR, Callaghan TV, Press MC, Aerts R (2011) Traits explain the responses of a sub-arctic Collembola community to climate manipulation. Soil Biology and Biochemistry, 43, 377-384.

Marino P, Raguso R, Goffinet B (2009) The ecology and evolution of fly dispersed dung mosses (Family Splachnaceae): Manipulating insect behaviour through odour and visual cues. Symbiosis, 47, 61-76.

Marion G, Henry G, Freckman D et al. (1997) Open,Äêtop designs for manipulating field temperature in high,Äêlatitude ecosystems. Global Change Biology, 3, 20-32.

Marquez LM, Redman RS, Rodriguez RJ, Roossinck MJ (2007) A virus in a fungus in a plant: three-way symbiosis required for thermal tolerance. Science, 315, 513-515.

Martindale JL, Holbrook NJ (2002) Cellular response to oxidative stress: Signaling for suicide and survival. Journal of Cellular Physiology, 192, $1-15$.

Martínez C, Pons E, Prats G, León J (2004) Salicylic acid regulates flowering time and links defence responses and reproductive development. The Plant Journal, 37, 209-217.

McDaniel SF (2005) Genetic Correlations Do Not Constrain the Evolution of Sexual Dimorphism in the Moss Ceratodon purpureus. Evolution, 59, 2353-2361.

McDaniel SF, Atwood J, Burleigh JG (2013a) Recurrent evolution of dioecy in bryophytes. Evolution, 67, 567-572.

McDaniel SF, Neubig KM, Payton AC, Quatrano RS, Cove DJ (2013b) Recent gene capture on the UV sex chromosomes of the moss, Ceratodon purpureus. Evolution.

McDaniel SF, Shaw AJ (2005) Selective sweeps and intercontinental migration in the cosmopolitan moss Ceratodon purpureus (Hedw.) Brid. Molecular Ecology, 14, 1121-1132. 
McDaniel SF, Willis JH, Shaw AJ (2007) A linkage map reveals a complex basis for segregation distortion in an interpopulation cross in the moss Ceratodon purpureus. Genetics, 176, 2489.

McGill BJ, Enquist BJ, Weiher E, Westoby M (2006) Rebuilding community ecology from functional traits. Trends in Ecology \& Evolution, 21, 178185.

McGinnis LK, Zhu L, Lawitts JA, Bhowmick S, Toner M, Biggers JD (2005) Mouse Sperm Desiccated and Stored in Trehalose Medium Without Freezing. Biology of Reproduction, 73, 627-633.

McMillan M, Shepherd A, Sundal A et al. (2014) Increased ice losses from Antarctica detected by CryoSat-2. Geophysical Research Letters, 2014GL060111.

Meredith MP, King JC (2005) Rapid climate change in the ocean west of the Antarctic Peninsula during the second half of the 20th century. Geophysical Research Letters, 32, L19604.

Meyer H, Santarius KA (1998) Short term thermal acclimation and heat tolerance of gametophytes of mosses. Oecologia, 115, 1-8.

Michalakis Y, Excoffier L (1996) A Generic Estimation of Population Subdivision Using Distances Between Alleles With Special Reference for Microsatellite Loci. Genetics, 142, 1061-1064.

Minchin P (1987) An evaluation of the relative robustness of techniques for ecological ordination. In: Theory and models in vegetation science. (eds Prentice IC, Maarel E) pp 89-107. Springer Netherlands.

Mishler BD (1990) Reproductive biology and species distinctions in the moss genus Tortula, as represented in Mexico. Systematic Botany, 15, 86-97.

Mishler BD, Churchill SP (1985) Transition to a land flora: phylogenetic relationships of the green algae and bryophytes. Cladistics, 1, 305-328.

Molau U, Parsons AN, Wookey PA, Welker JM, Robinson CH (1997) Responses of Dryas octopetala to ITEX environmental manipulations: a synthesis with circumpolar comparisons. Global Change Biology, 3, 61.

Møller IM (2001) Plant mitochondria and oxidative stress: Electron Transport, NADPH Turnover, and Metabolism of Reactive Oxygen Species. Annual Review of Plant Physiology and Plant Molecular Biology, 52, 561-591.

Montes-Hugo M, Doney SC, Ducklow HW, Fraser W, Martinson D, Stammerjohn SE, Schofield O (2009) Recent changes in phytoplankton communities associated with rapid regional climate change along the western Antarctic Peninsula. Science, 323, 1470-1473.

Moore JC, Tripp BB, Simpson RT, Coleman DC (2000) Springtails in the classroom - Collembola as model organisms for inquiry-based laboratories. American Biology Teacher, 62, 512-519. 
Morton BB, Forsythe AB (1974) Robust Tests for the Equality of Variances. Journal of the American Statistical Association, 69, 364-367.

Muffler LJ, Nehring NL, Truesdell AH, Janik CJ, Clynne MA, Thompson JM (1983) The Lassen geothermal system. Menlo Park, U.S. Geological Survey.

Muggoch H, Walton J (1942) On the dehiscence of the antheridium and the part played by surface tension in the dispersal of spermatocyctes in Bryophyta. Proceedings of the Royal Society B-Biological Sciences, 130, 448-461.

Nabe H, Funabiki R, Kashino Y, Koike H, Satoh K (2007) Responses to Desiccation Stress in Bryophytes and an Important Role of Dithiothreitol-Insensitive Non-Photochemical Quenching Against Photoinhibition in Dehydrated States. Plant and Cell Physiology, 48, 1548-1557.

Nash MA, Griffin PC, Hoffmann AA (2013) Inconsistent responses of alpine arthropod communities to experimental warming and thermal gradients. Climate Research, 55, 227-237.

Nickrent DL, Parkinson CL, Palmer JD, Duff RJ (2000) Multigene phylogeny of land plants with special reference to bryophytes and the earliest land plants. Molecular Biology and Evolution, 17, 1885-1895.

Ochyra R (1998) The moss flora of King George Island, Antarctica, Polish Academy of Sciences, W. Szafer Institute of Botany.

Ochyra R, Lewis Smith RI, Bednarek-Ochyra H (2008) The illustrated moss flora of Antarctica, Cambridge University Press.

Oechel WC, Van Cleve K (1986) The Role of Bryophytes in Nutrient Cycling in the Taiga. In: Forest Ecosystems in the Alaskan Taiga. (eds Van Cleve K, Chapin FS, Iii, Flanagan PW, Viereck LA, Dyrness CT) pp 121-137. Springer New York.

Ohlson M, Økland RH, Nordbakken J-F, Dahlberg B (2001) Fatal interactions between Scots pine and Sphagnum mosses in bog ecosystems. Oikos, 94, 425-432.

Oksanen J, Kindt R, Legendre P, O,Äôhara B, Stevens MHH, Oksanen MJ, Suggests M (2007) The vegan package. Community ecology package.

Oliver MJ, Bewley JD (1984a) Desiccation and ultrastructure in bryophytes. Advances in Bryology, 2, 91-131.

Oliver MJ, Bewley JD (1984b) Plant desiccation and protein synthesis: VI. Changes in protein synthesis elicited by desiccation of the moss Tortula ruralis are effected at the translational level. Plant Physiology, 74, 923-927.

Oliver MJ, Tuba Z, Mishler BD (2000) The evolution of desiccation tolerance in land plants. Plant Ecology, 16, 85-100.

Oliver MJ, Velten J, Mishler BD (2005) Desiccation Tolerance in Bryophytes: A Reflection of the Primitive Strategy for Plant Survival in 
Dehydrating Habitats? Integrative and Comparative Biology, 45, 788799.

Oliver MJ, Velten J, Wood AJ (2000b) Bryophytes as experimental models for the study of environmental stress tolerance: Tortula ruralis and desiccation-tolerance in mosses. Plant Ecology, 151, 73-84.

Oliver MJ, Wood AJ, O'mahony P (1997) How some plants recover from vegetative desiccation: A repair based strategy. Acta Physiologiae Plantarum, 19, 419-425.

Opelt K, Chobot V, Hadacek F, Schönmann S, Eberl L, Berg G (2007) Investigations of the structure and function of bacterial communities associated with Sphagnum mosses. Environmental Microbiology, 9, 2795-2809.

Osler GH, Sommerkorn M (2007) Toward a complete soil C and N cycle: incorporating the soil fauna. Ecology, 88, 1611-1621.

Ostle N, Briones M, Ineson P, Cole L, Staddon P, Sleep D (2007) Isotopic detection of recent photosynthate carbon flow into grassland rhizosphere fauna. Soil Biology and Biochemistry, 39, 768-777.

Pammenter NW, Berjak P (1999) A review of recalcitrant seed physiology in relation to desiccation-tolerance mechanisms. Seed Science Research, 9, 13-37.

Pandey KD, Kashyap AK, Gupta RK (1992) Nitrogen fixation by cyanobacteria associated with moss communities in Schirmacher Oasis, Antarctica. Israel Journal of Botany, 41, 187-198.

Pankow J, Luo W, Melnychenko A et al. (2011) Volatilizable biogenic organic compounds (VBOCs) with two dimensional gas chromatography-time of flight mass spectrometry (GCxGC-TOFMS): sampling methods, VBOC complexity, and chromatographic retention data. Atmospheric Measurement Techniques Discussions, 4, 3647-3684.

Paolillo DJ, Jr. (1975) The Release of Sperms from the Antheridia of Polytrichum juniperinum Hedw. New Phytologist, 74, 287-293.

Paolillo DJ, Jr. (1977) On the Release of Sperms in Atrichum. American Journal of Botany, 64, 81-85.

Paolillo DJ, Jr. (1979) On the Lipids of the Sperm Masses of Three Mosses. The Bryologist, 82, 93-96.

Paolillo DJ, Jr. (1981) The Swimming Sperms of Land Plants. BioScience, 31, 367-373.

Park J-H, Day TA, Strauss S, Ruhland CT (2007) Biogeochemical pools and fluxes of carbon and nitrogen in a maritime tundra near penguin colonies along the Antarctic Peninsula. Polar Biology, 30, 199-207.

Peakall R, Smouse PE (2012) GenAlEx 6.5: genetic analysis in Excel. Population genetic software for teaching and research: an update. Bioinformatics, 28, 2537-2539. 
Peakall ROD, Smouse PE (2006) Genalex 6: genetic analysis in Excel. Population genetic software for teaching and research. Molecular Ecology Notes, 6, 288-295.

Peker D, Dolan EL (2014) Guiding student's scientific Ppractice: distinct and common roles for teachers and scientists. SAGE Open, 4.

Perera RM, Marriott PJ, Galbally IE (2002) Headspace solid-phase microextraction: comprehensive two-dimensional gas chromatography of wound induced plant volatile organic compound emissions. Analyst, 127, 1601-1607.

Pickett ST, Parker VT, Fiedler PL (1992) The new paradigm in ecology: implications for conservation biology above the species level. In: Conservation Biology. pp 65-88. Springer.

Pope C, Ziebland S, Mays N (2000) Analysing qualitative data. Bmj, 320, 114116.

Potts M (1994) Desiccation tolerance of prokaryotes. Microbiol. Mol. Biol. Rev., 58, 755-805.

Proctor M (2001) Patterns of desiccation tolerance and recovery in bryophytes. Plant Growth Regulation, 35, 147-156.

Proctor MCF, Oliver MJ, Wood AJ, Alpert P, Stark LR, Cleavitt NL, Mishler BD (2007) Desiccation-tolerance in bryophytes: a review. Bryologist, 110, 595-621.

Proctor MCF, Pence VC (2002) Vegetative Tissues: Bryophytes, vascular resurrection plants, and vegetative propogules. In: Desiccation and survival in plants: Drying without dying. (ed Pritchard MBaHW) pp 207-237. Wallingford, Oxon, CABI Publishing.

Proctor MCF, Smirnoff N (2000) Rapid recovery of photosystems on rewetting desiccation-tolerant mosses: chlorophyll fluorescence and inhibitor experiments. Journal of Experimental Botany, 51, 1695-1704.

Quinn H, Schweingruber H, Keller T (2012) A Framework for K-12 Science Education: Practices, Crosscutting Concepts, and Core Ideas, The National Academies Press.

R Development Core Team (2006) R: A language and environment for statistical computing. Vienna, Austria., R Foundation for Statistical Computing.

Raguso RA (2008) Wake Up and Smell the Roses: The Ecology and Evolution of Floral Scent. Annual Review of Ecology, Evolution, and Systematics, 39, 549-569.

Rao CR (1982) Diversity and dissimilarity coefficients: a unified approach. Theoretical Population Biology, 21, 24-43.

Raspotnig G, Krisper G, Schuster R, Fauler G, Leis HJ (2005) Volatile exudates from the oribatid mite, Platynothrus peltifer. Journal of Chemical Ecology, 31, 419-430. 
Renner SS, Ricklefs RE (1995) Dioecy and its correlates in the flowering plants. American Journal of Botany, 82, 596-606.

Renzaglia KS, Rasch EM, Pike LM (1995) Estimates of nulcear DNA content in bryohpyte sperm cells-phylogentic considerations. American Journal of Botany, 82, 18-25.

Reysenbach A-L, Shock E (2002) Merging genomes with geochemistry in hydrothermal ecosystems. Science, 296, 1077-1082.

Rice SK, Aclander L, Hanson DT (2008) Do bryophyte shoot systems function like vascular plant leaves or canopies? Functional trait relationships in Sphagnum mosses (Sphagnaceae). Am. J. Bot., 95, 1366-1374.

Rinnan R, Rousk J, Yergeau E, Kowalchuk GA, Baath E (2009) Temperature adaptation of soil bacterial communities along an Antarctic climate gradient: predicting responses to climate warming. Global Change Biology, 15, 2615-2625.

Robarts RD, Zohary T (1987) Temperature effects on photosynthetic capacity, respiration, and growth rates of bloom,Äêforming cyanobacteria. New Zealand Journal of Marine and Freshwater Research, 21, 391-399.

Robinson CH, Wookey PA, Lee JA, Callaghan TV, Press MC (1998) Plant commuity reponses to simulated environmental change at a high arctic polar semi-desert. Ecology, 79, 856-866.

Robinson SA, Wasley J, Popp M, Lovelock CE (2000) Desiccation tolerance of three moss species from continental Antarctica. Australian Journal of Plant Physiology, 27, 379-388.

Robinson SA, Wasley J, Tobin AK (2003) Living on the edge, plants and global change in continental and maritime Antarctica. Global Change Biology, 9, 1681-1717.

Rodriguez RJ, Henson J, Van Volkenburgh E et al. (2008) Stress tolerance in plants via habitat-adapted symbiosis. The ISME journal, 2, 404-416.

Rosenstiel TN, Eppley SM (2009) Long-lived sperm in the geothermal bryophyte Pohlia nutans. Biol Lett, 5, 857-860.

Rosenstiel TN, Shortlidge EE, Melnychenko AN, Pankow JF, Eppley SM (2012) Sex-specific volatile compounds influence microarthropodmediated fertilization of moss. Nature, 489, 431-433.

Rowntree JK, Duckett JG, Mortimer CL, Ramsay MM, Pressel S (2007) Formation of Specialized Propagules Resistant to Desiccation and Cryopreservation in the Threatened Moss Ditrichum plumbicola (Ditrichales, Bryopsida). Annals of Botany, 100, 483-496.

Royles J, Amesbury MJ, Convey P, Griffiths H, Hodgson DA, Leng MJ, Charman DJ (2013) Plants and Soil Microbes Respond to Recent Warming on the Antarctic Peninsula. Current Biology, 23, 1702-1706.

Rustad L, Campbell J, Marion G et al. (2001) A meta-analysis of the response of soil respiration, net nitrogen mineralization, and aboveground plant growth to experimental ecosystem warming. Oecologia, 126, 543-562. 
Rütten D, Santarius KA (1992) Relationship between frost tolerance and sugar concentration of various bryophytes in summer and winter. Oecologia, 91, 260-265.

Sadaka-Laulan N, Ponge JF, Roquebert MF, Bury E, Boumezzough A (1998) Feeding preferences of the collembolan Onychiurus sinensis for fungi colonizing holm oak litter (Quercus rotundifolia Lam.). European Journal of Soil Biology, 34, 179-188.

Sairam RK, Rao KV, Srivastava G (2002) Differential response of wheat genotypes to long term salinity stress in relation to oxidative stress, antioxidant activity and osmolyte concentration. Plant Science, 163, 1037-1046.

Saito C, Nagata N, Sakai A, Mori K, Kuroiwa H, Kuroiwa T (2002) Angiosperm species that produce sperm cell pairs or generative cells with polarized distribution of DNA-containing organelles. Sexual Plant Reproduction, 15, 167-178.

SAS Institute (2012) JMP for Windows. Release 10.0.0. Cary, N.C.

Schiestl FP (2010) The evolution of floral scent and insect chemical communication. Ecology letters, 13, 643-656.

Schonbek MW, Bewley JD (1981) Responses of the moss Tortula ruralis to desiccation treatments: I. Effects of minimum water content and ratesof dehydration and rehydration. Canadian Journal of Botany, 59, 2698-2706.

Schreiber U, Bilger W, Neubauer C (1995) Chlorophyll fluorescence as a nonintrusive indicator for rapid assessment of in vivo photosynthesis. In: Ecophysiology of photosynthesis. pp 49-70. Springer.

Schuman H, Presser S (1996) Questions and answers in attitude surveys: Experiments on question form, wording, and context, Sage.

Seel WE, F. Hendry GA, Lee JA (1992a) Effects of Desiccation on some Activated Oxygen Processing Enzymes and Anti-Oxidants in Mosses. Journal of Experimental Botany, 43, 1031-1037.

Seel WE, Hendry GaF, Lee JA (1992b) The Combined Effects of Desiccation and Irradiance on Mosses from Xeric and Hydric Habitats. Journal of Experimental Botany, 43, 1023-1030.

Selkoe KA, Toonen RJ (2006) Microsatellites for ecologists: a practical guide to using and evaluating microsatellite markers. Ecology letters, 9, 615629.

Shaw AJ, Beer SC (1999) Life history variation in gametophyte populations of the moss Ceratodon purpureus (Ditrichaceae). American Journal of Botany, 86, 512-521.

Shaw AJ, Gaughan JF (1993) Control of sex-ratios in haploid populations of the moss, Ceratodon purpureus. American Journal of Botany, 80, 584591. 
Shaw AJ, Goffinet B (2000) Bryophyte Biology, Cambridge, UK, Cambridge University Press.

Shaw J (1986) A new approach to the experimental propagation of bryophytes. Taxon, 671-675.

Shortlidge EE, Rosenstiel TN, Eppley SM (2012) Tolerance to environmental desiccation in moss sperm. New Phytologist, 194, 741-750.

Showers DW (1982) The mosses of Lassen Volcanic National Park, California. Bryologist, 324-328.

Siering PL, Clarke JM, Wilson MS (2006) Geochemical and biological diversity of acidic, hot springs in Lassen Volcanic National Park. Geomicrobiology Journal, 23, 129-141.

Silberglied RE, Shepherd JG, Dickinson JL (1984) Eunuchs- the role of apyrene sperm in Lepidoptera. American Naturalist, 123, 255-265.

Skotnicki M, Bargagli R, Ninham J (2002) Genetic diversity in the moss Pohlia nutans on geothermal ground of Mount Rittmann, Victoria Land, Antarctica. Polar Biology, 25, 771-777.

Skotnicki M, Mackenzie A, Ninham J, Selkirk P (2004) High levels of genetic variability in the moss Ceratodon purpureus from continental Antarctica, subantarctic Heard and Macquarie Islands, and Australasia. Polar Biology, 27, 687-698.

Smirnoff N (1992) The carbohydrates of bryophytes in relation to desiccation tolerance. Journal Of Bryology, 17, 185-191.

Smith CW (1981) Bryophytes and lichens of the Puhima Geothermal Area, Hawaii Volcanoes National Park. The Bryologist, 84, 457-466.

Smith R (2005) The bryophyte flora of geothermal habitats on Deception Island, Antarctica. Journal of the Hattori Botanical Laboratory, 97, 233-248.

Smith R, Convey P (2002a) Enhanced sexual reproduction in bryophytes at high latitudes in the maritime Antarctic. Journal Of Bryology, 24, 107117.

Smith RIL (1994) Vascular plants as bioindicators of regional warming in Antarctica. Oecologia, 99, 322-328.

Smith RL, Convey P (2002b) Enhanced sexual reproduction in bryophytes at high latitudes in the maritime Antarctic. Journal Of Bryology, 24, 107117.

Snyder JC (2005) Virus dynamics, archael populations, and water chemistry or three acidic hot springs in Yellowstone National Park. Unpublished PhD Montana State University, Bozeman.

Southwood TRE, Henderson PA (2009) Ecological methods, John Wiley \& Sons.

Southworth D, Cresti M (1997) Comparison of flagellated and nonflagellated sperm in plants. American Journal of Botany, 84, 1301. 
Spence JR, Niemela JK (1994) Sampling Carabid assemblages with pitfall traps: the madness and the method. The Canadian Entomologist, 126, 881-894.

Srivastava DS, Kolasa J, Bengtsson J et al. (2004) Are natural microcosms useful model systems for ecology? Trends in Ecology \& Evolution, 19, 379-384.

Staaden S, Milcu A, Rohlfs M, Scheu S (2011) Olfactory cues associated with fungal grazing intensity and secondary metabolite pathway modulate Collembola foraging behaviour. Soil Biology and Biochemistry, 43, 1411-1416.

Staddon P, Lindo Z, Crittenden PD, Gilbert F, Gonzalez A (2010) Connectivity, non-random extinction and ecosystem function in experimental metacommunities. Ecology letters, 13, 543-552.

Stark LK, Mcletchie DN, Mishler BD (2005) Sex expression, plant size, and spatial segregation of the sexes across a stress gradient in the desert moss Syntrichia caninervis. Bryologist, 108, 183-193.

Stark LR (2002) Skipped reproductive cycles and extensive sporophyte abortion in the desert moss Tortula inermis correspond to unusual rainfall patterns. Canadian Journal of Botany, 80, 533-542.

Stark LR, Brinda JC, Mcletchie DN (2009) An experimental demonstration of the cost of sex and a potential resource limitation on reproduction in the moss Pterygoneurum (Pottiaceae). American Journal of Botany, 96, $1712-1721$.

Stark LR, Mcletchie DN, Eppley SM (2010) Sex ratios and the shy male hypothesis in Bryum argenteum (Bryaceae). Bryologist, 113, 788-797.

Stark LR, Mishler BD, Mcletchie DN (2000) The cost of realized sexual reproduction: Assessing patterns of reproductive allocation and sporophyte abortion in a desert moss. American Journal of Botany, 87, 1599-1608.

Steidle JL, Schöller M (1997) Olfactory host location and learning in the granary weevil parasitoid Lariophagus distinguendus (Hymenoptera: Pteromalidae). Journal of insect behavior, 10, 331-342.

Stout RG, Al-Niemi TS (2002) Heat-tolerant Flowering Plants of Active Geothermal Areas in Yellowstone National Park. Annals of Botany, 90, 259-267.

Strack F, Martin LL (1987) Thinking, judging, and communicating: A process account of context effects in attitude surveys. In: Social information processing and survey methodology. pp 123-148. Springer.

Suding KN, Lavorel S, Chapin FS et al. (2008) Scaling environmental change through the community-level: a trait-based response-and-effect framework for plants. Global Change Biology, 14, 1125-1140. 
Sun WQ, Irving TC, Leopold AC (1994) The role of sugar, vitrification and membrane phase-transition in seed desiccation tolerance. Physiologia Plantarum, 90, 621-628.

Sunday JM, Bates AE, Dulvy NK (2011) Global analysis of thermal tolerance and latitude in ectotherms. Proceedings of the Royal Society B: Biological Sciences, 278, 1823-1830.

Szövényi P, Devos N, Weston DJ et al. (2014) Efficient Purging of Deleterious Mutations in Plants with Haploid Selfing. Genome Biology and Evolution, 6, 1238-1252.

Szövényi P, Ricca M, Shaw AJ (2009) Multiple paternity and sporophytic inbreeding depression in a dioicous moss species. Heredity, 103, 394403.

Tanner KD (2011) Reconsidering "what works". CBE-Life Sciences Education, 10, 329-333.

Taylor LP, Hepler PK (1997) Pollen germination and tube growth. Annual Review of Plant Biology, 48, 461-491.

Team R Core (2013) R: A language and environment for statistical computing. R Foundation for Statistical Computing, Vienna, Austria. URL http://www.R-project.org/. ISBN 3-900051-07-0.

Tercek MT, Whitbeck JL (2004) Heat avoidance life history strategy controls the distribution of geothermal Agrostis in Yellowstone. Ecology, 85, 1955-1966.

Thimm T, Larink O (1995) Grazing preferences of some collembola for endomycorrhizal fungi. Biology and Fertility of Soils, 19, 266-268.

Thomas JW (2000) A review of research on project-based learning.

Thompson JM (1982) Preliminary chemical studies of thermal waters in Lassen Volcanic National Park and vicinities. Geothermal Resources Council, Transactions, 6, 115-118.

Thompson JM (1983) Chemical analyses of thermal and nonthermal springs in Lassen Volcanic Park and vicinity, California. Menlo Park, United States Geographical Survey.

Thornton LE, Keren N, Ohad I, Pakrasi HB (2005) Physcomitrella patens and Ceratodon purpureus, mosses as model organisms in photosynthesis studies. Photosynthesis Research, 83, 87-96.

Thornton RK, Sokoloff DR (1998) Assessing student learning of Newton,Äôs laws: The force and motion conceptual evaluation and the evaluation of active learning laboratory and lecture curricula. American Journal of Physics, 66, 338-352.

Till-Bottraud I, Joly D, Lachaise D, Snook RR (2005) Pollen and sperm heteromorphism: convergence across kingdoms? Journal of Evolutionary Biology, 18, 1-18.

Tilman D, El Haddi A (1992) Drought and biodiversity in grasslands. Oecologia, 89, 257-264. 
Turetsky MR (2003) The role of bryophytes in carbon and nitrogen cycling. The Bryologist, 106, 395-409.

Turetsky MR, Bond-Lamberty B, Euskirchen E, Talbot J, Frolking S, Mcguire AD, Tuittila E-S (2012) The resilience and functional role of moss in boreal and arctic ecosystems. New Phytologist, 196, 49-67.

Usher MB, Booth RG (1984) Arthropod Communities in a Maritime Antarctic Moss-Turf Habitat: Three-Dimensional Distribution of Mites and Collembola. Journal of Animal Ecology, 53, 427-441.

Van Dam N, Poppy G (2008) Why plant volatile analysis needs bioinformatics: detecting signal from noise in increasingly complex profiles. Plant Biology, 10, 29-37.

Van Der Velde M, Bijlsma R (2000) Amount and structure of intra-and interspecific genetic variation in the moss genus Polytrichum. Heredity, 85, 328-337.

Van Der Velde M, During HJ, Van Der Zande L, Bijlsma R (2001a) The reproductive biology of Polytrichum formosum: clonal structure and paternity revealed by microsatellites. Molecular Ecology, 10.

Van Der Velde M, Van De Zande L, Bijlsma R (2001b) Genetic structure of Polytrichum formosum in relation to the breeding system as revealed by microsatellites. Journal of Evolutionary Biology, 14, 288-295.

Van Der Wal R, Brooker RW (2004) Mosses mediate grazer impacts on grass abundance in arctic ecosystems. Functional Ecology, 18, 77-86.

Van Lipzig N, King J, Lachlan,Äêcope T, Van Den Broeke M (2004) Precipitation, sublimation, and snow drift in the Antarctic Peninsula region from a regional atmospheric model. Journal of Geophysical Research: Atmospheres (1984,Ä̈2012), 109.

Van Look KJW, Dzyuba B, Cliffe A, Koldewey HJ, Holt WV (2007) Dimorphic sperm and the unlikely route to fertilisation in the yellow seahorse. Journal of Experimental Biology, 210, 432-437.

Vanderpoorten A, Goffinet B (2009) Introduction to Bryophytes, Cambridge, Cambridge Unversity Press.

Varga S, Kytoviita MM (2008) Sex-specific responses to mycorrhiza in a dioecious species. American Journal of Botany, 95, 1225-1232.

Vaughan D, Marshall G, Connolley W et al. (2003) Recent Rapid Regional Climate Warming on the Antarctic Peninsula. Climatic Change, 60, 243-274.

Vaughan DG (2006) Recent trends in melting conditions on the Antarctic Peninsula and their implications for ice-sheet mass balance and sea level. Arctic, Antarctic, and Alpine Research, 38, 147-152.

Verhoef HA, Nagelkerke CJ, Joosse ENG (1977) Aggregation pheromones in Collembola. Journal of Insect Physiology, 23, 1009-1013. 
Voigt CC, Caspers B, Speck S (2005) Bats, bacteria, and bat smell: Sexspecific diversity of microbes in a sexually selected scent organ. Journal of Mammalogy, 86, 745-749.

Wahid A, Gelani S, Ashraf M, Foolad M (2007) Heat tolerance in plants: an overview. Environmental and Experimental Botany, 61, 199-223.

Wahren CÄ, Walker M, Bret,Äêharte M (2005) Vegetation responses in Alaskan arctic tundra after 8 years of a summer warming and winter snow manipulation experiment. Global Change Biology, 11, 537-552.

Waite M, Sack L (2010) How does moss photosynthesis relate to leaf and canopy structure? Trait relationships for 10 Hawaiian species of contrasting light habitats. New Phytologist, 185, 156-172.

Walker JJ, Spear JR, Pace NR (2005) Geobiology of a microbial endolithic community in the Yellowstone geothermal environment. Nature, 434, 1011-1014.

Walker MD, Wahren CH, Hollister RD et al. (2006) Plant community responses to experimental warming across the tundra biome. Proceedings of the National Academy of Sciences of the United States of America, 103, 1342-1346.

Wall DH, Virginia RA (1999) Controls on soil biodiversity: insights from extreme environments. Applied Soil Ecology, 13, 137-150.

Walter DE (1987) Trophic behavior of" mycophagous" microarthropods. Ecology, 68, 226-229.

Walther G-R, Post E, Convey P et al. (2002) Ecological responses to recent climate change. Nature, 416, 389-395.

Ward DM, Ferris MJ, Nold SC, Bateson MM (1998) A natural view of microbial biodiversity within hot spring cyanobacterial mat communities. Microbiology and Molecular Biology Reviews, 62, 13531370.

Weeks RD, Mcintyre NE (1997) A comparison of live versus kill pitfall trapping techniques using various killing agents. Entomologia Experimentalis et Applicata, 82, 267-273.

Weir BS, Cockerham CC (1984) Estimating F-statistics for the analysis of population structure. Evolution, 1358-1370.

Weterings K, Russell SD (2004) Experimental analysis of the fertilization process. Plant Cell, 16, S107-S118.

Whitham TG, Bailey JK, Schweitzer JA et al. (2006) A framework for community and ecosystem genetics: from genes to ecosystems. Nature Reviews Genetics, 7, 510-523.

Wilson JA, Coxson DS (1999) Carbon flux in a subalpine spruce-fir forest: pulse release from Hylocomium splendens feather-moss mats. Canadian Journal of Botany, 77, 564-569.

Wilson PJ, Provan J (2003) Effect of habitat fragmentation on levels and patterns of genetic diversity in natural populations of the peat moss 
Polytrichum commune. Proceedings of the Royal Society of London. Series B: Biological Sciences, 270, 881-886.

Winston PW, Bates DH (1960) Saturated Solutions For the Control of Humidity in Biological Research. Ecology, 41, 232-237.

Womersley C, Ching C (1989) Natural dehydration regimes as a prerequisite for the succesful induciton of anhydrobiosis in the nematode Rotylenchulus reniformis. Journal of Experimental Biology, 143, 359372.

Wood AJ, Oliver MJ (2004) Molecular biology and genomics of the desiccation-tolerant moss Tortula ruralis. New frontiers in bryology:

Physiology, molecular biology and functional genomics, 71-90.

Wright JP, Jones CG (2006) The concept of organisms as ecosystem engineers ten years on: progress, limitations, and challenges. BioScience, 56, 203209.

Wyatt R (1977) Spatial pattern and gamete dispersal distances in Atrichum angustatum, a dioicous moss. Bryologist, 284-291.

Wyatt R, Anderson LE (1984) Breeding systems in bryophytes. In: The Experimental Biology of Bryophytes. (eds Dyer AF, Duckett JG) pp 3964. London, Academic Press.

Wyatt R, Odrzykoski IJ, Stoneburner A (1989) High levels of genetic variability in the haploid moss Plagiomnium ciliare. Evolution, 43, 1085-1096.

Xu J, Ke X, Krogh PH, Wang Y, Luo YÄ, Song J (2009) Evaluation of growth and reproduction as indicators of soil metal toxicity to the Collembolan, Sinella curviseta. Insect Science, 16, 57-63.

Yund PO (2000) How severe is sperm limitation in natural populations of marine free-spawners? Trends in Ecology \& Evolution, 15, 10-13.

Ziegler H, Kaiser K, Lipp J (1988) Sucrose in the archegonium exudate of the moss Bryum capillare; Hedw. Naturwissenschaften, 75, 203-203.

Zinn KE, Tunc-Ozdemir M, Harper JF (2010) Temperature stress and plant sexual reproduction: uncovering the weakest links. Journal of Experimental Botany, 61, 1959-1968. 


\section{Appendices}

\section{List of Tables and Figures}

\section{Tables}

Table A.A1 Volatile organic compounds detected via GCxGC-TOFMS headspace analysis of intact male and female shoots of Ceratodon purpureus....229 Table A.A2 ANOVA and column statistics for variables measured by Springtail/Water Spray treatment..........................................258 Table A.B1 Tukey's HSD post hoc analysis results for mixed model ANOVA on the effects of strain by treatment on total sporophyte production..........277 Table A.C.1 Results of mixed-model ANOVA and post hoc Tukey's HSD analyses on sporophyte distance from center and sporophyte height.........300

\section{Figures}

A.A1 Male and female moss volatile profile composition.....................259 A.A2 Moss Volatile Organic Compounds from C. purpureus individuals by sex and IUPAC compound class.............................................260 A.A3 Volatile profiles binned by IUPAC compound class of five representative male and female $C$. purpureus mosses.........................261 A.A4 Microcosm canopy measurements.....................................262

A.B1 Growth environments of $P$. patens....................................278

A.B2 Total number of sporophytes produced in microcosm arrays...........279 A.B3 Three $P$. patens strains responses to four fertilization

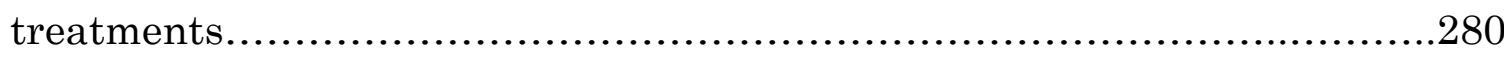


A.C1 Chlorophyll content from bryospheres at two time points...............301

A.C2 Fv/Fm values of C. purpureus canopies after one year of growth......302

A.C3 Total sporophytes produced in bryospheres after 16 months...........303

A.C4 Sporophyte distance from center and height............................304

A.C5 Sporophytes produced by each male grouping............................305

A.C6 Correlation between sporophyte distance from center and height.....306 


\section{Appendix A}

Supplemental materials to Chapter 3: Sex-specific volatile compounds influence microarthropod-mediated fertilization of moss

Table A.A1 Volatile organic compounds detected via GCxGC-TOFMS head-space analysis of intact male and female shoots of Ceratodon purpureus. Table depicts number of samples where a compound was observed (N), CAS number, compound name, molecular formula, IAUPAC class categorization, and primary and secondary retention times (s), respectively. 


\begin{tabular}{|c|c|c|c|c|c|}
\hline $\mathbf{N}$ & CAS & Name & $\begin{array}{c}\text { Form } \\
\text { ula }\end{array}$ & Class & $\begin{array}{c}\text { Rt1, } \\
\text { Rt2 } \\
\text { (s) }\end{array}$ \\
\hline 3 & $\begin{array}{l}112- \\
05-0\end{array}$ & Nonanoic acid & $\begin{array}{c}\mathrm{C}_{9} \mathrm{H}_{18} \\
\mathrm{O}_{2}\end{array}$ & Acid & $\begin{array}{c}1423 . \\
88 \\
0.304\end{array}$ \\
\hline 2 & $\begin{array}{l}594- \\
61-6\end{array}$ & Propanoic acid, 2-hydroxy-2-methyl- & $\begin{array}{c}\mathrm{C}_{4} \mathrm{H}_{8} \\
\mathrm{O}_{3}\end{array}$ & Acid & $\begin{array}{c}1303 . \\
89 \\
1.544\end{array}$ \\
\hline 1 & $\begin{array}{l}143- \\
07-7\end{array}$ & Dodecanoic acid & $\begin{array}{c}\mathrm{C}_{12} \\
\mathrm{H}_{24} \mathrm{O}_{2}\end{array}$ & Acid & $\begin{array}{c}1907 . \\
83 \\
4.000\end{array}$ \\
\hline 1 & $\begin{array}{l}142- \\
62-1\end{array}$ & Hexanoic acid & $\begin{array}{c}\mathrm{C}_{6} \mathrm{H}_{12} \\
\mathrm{O}_{2}\end{array}$ & Acid & $\begin{array}{c}1031 . \\
91 \\
3.326\end{array}$ \\
\hline 1 & $\begin{array}{c}5123 \\
45\end{array}$ & Hexanoic acid, 3,5,5-trimethyl- & $\begin{array}{c}\mathrm{C}_{9} \mathrm{H}_{18} \\
\mathrm{O}_{2}\end{array}$ & Acid & $\begin{array}{c}2007 . \\
82 \\
1.261\end{array}$ \\
\hline 1 & $\begin{array}{c}57- \\
10-3\end{array}$ & n-Hexadecanoic acid & $\begin{array}{c}\mathrm{C}_{16} \\
\mathrm{H}_{32} \mathrm{O}_{2}\end{array}$ & Acid & $\begin{array}{c}2671 . \\
75 \\
2.138\end{array}$ \\
\hline 1 & $\begin{array}{l}124- \\
07-2\end{array}$ & Octanoic Acid & $\begin{array}{c}\mathrm{C}_{8} \mathrm{H}_{16} \\
\mathrm{O}_{2}\end{array}$ & Acid & $\begin{array}{c}1275 . \\
89 \\
0.356\end{array}$ \\
\hline 1 & $\begin{array}{l}144- \\
62-7\end{array}$ & Oxalic acid & $\begin{array}{c}\mathrm{C}_{2} \mathrm{H}_{2} \\
\mathrm{O}_{4}\end{array}$ & Acid & $\begin{array}{c}263.9 \\
92 \\
2.594\end{array}$ \\
\hline 1 & $\begin{array}{c}75- \\
98-9\end{array}$ & Propanoic acid, 2,2-dimethyl- & $\begin{array}{c}\mathrm{C}_{5} \mathrm{H}_{10} \\
\mathrm{O}_{2}\end{array}$ & Acid & $\begin{array}{c}667.9 \\
51, \\
1.525\end{array}$ \\
\hline 1 & $\begin{array}{l}544- \\
63-8\end{array}$ & Tetradecanoic acid & $\begin{array}{c}\mathrm{C}_{14} \\
\mathrm{H}_{28} \mathrm{O}_{2}\end{array}$ & Acid & $\begin{array}{c}2239 . \\
79 \\
0.152\end{array}$ \\
\hline 1 & $\begin{array}{l}112- \\
37-8\end{array}$ & Undecanoic acid & $\begin{array}{c}\mathrm{C}_{11} \\
\mathrm{H}_{22} \mathrm{O}_{2}\end{array}$ & Acid & $\begin{array}{c}2239 . \\
79 \\
0.020\end{array}$ \\
\hline 16 & $\begin{array}{l}616- \\
25-1\end{array}$ & 1-Penten-3-ol & $\begin{array}{c}\mathrm{C}_{5} \mathrm{H}_{10} \\
\mathrm{O}\end{array}$ & Alcohol & $\begin{array}{c}1095 . \\
91 \\
1.063\end{array}$ \\
\hline 16 & $\begin{array}{l}584- \\
02-1\end{array}$ & 3-Pentanol & $\begin{array}{c}\mathrm{C}_{5} \mathrm{H}_{12} \\
\mathrm{O}\end{array}$ & Alcohol & $\begin{array}{c}663.9 \\
52 \\
1.551\end{array}$ \\
\hline 15 & $\begin{array}{c}3391 \\
-86- \\
4\end{array}$ & 1-Octen-3-ol & $\begin{array}{c}\mathrm{C}_{8} \mathrm{H}_{16} \\
\mathrm{O}\end{array}$ & Alcohol & $\begin{array}{c}1059 . \\
91 \\
1.406\end{array}$ \\
\hline 9 & $\begin{array}{c}2029 \\
6- \\
29-1\end{array}$ & 3-Octanol & $\begin{array}{c}\mathrm{C}_{8} \mathrm{H}_{18} \\
\mathrm{O}\end{array}$ & Alcohol & $\begin{array}{c}1075 . \\
91 \\
1.287\end{array}$ \\
\hline 8 & $\begin{array}{l}924- \\
41-4\end{array}$ & 1,5-Hexadien-3-ol & $\begin{array}{c}\mathrm{C}_{6} \mathrm{H}_{10} \\
\mathrm{O}\end{array}$ & Alcohol & $\begin{array}{c}675.9 \\
5 \\
1.261\end{array}$ \\
\hline 8 & 5390 & 6-Hepten-3-ol, 4-methyl- & $\mathrm{C}_{8} \mathrm{H}_{16}$ & Alcohol & 1059. \\
\hline
\end{tabular}




\begin{tabular}{|c|c|c|c|c|c|}
\hline & $7-$ & & $\mathrm{O}$ & & 91 , \\
\hline & $71-4$ & & & & 1.327 \\
\hline 7 & $\begin{array}{c}1576 \\
-95- \\
0\end{array}$ & 2-Penten-1-ol, (Z)- & $\begin{array}{c}\mathrm{C}_{5} \mathrm{H}_{10} \\
\mathrm{O}\end{array}$ & Alcohol & $\begin{array}{c}1099 . \\
91, \\
1.102\end{array}$ \\
\hline 6 & $\begin{array}{c}1954 \\
9- \\
70-3\end{array}$ & 3-Heptanol, 2,2-dimethyl- & $\begin{array}{c}\mathrm{C}_{9} \mathrm{H}_{20} \\
\mathrm{O}\end{array}$ & Alcohol & $\begin{array}{c}1203 . \\
9, \\
1.841\end{array}$ \\
\hline 6 & $\begin{array}{l}928- \\
96-1\end{array}$ & 3-Hexen-1-ol, (Z)- & $\begin{array}{c}\mathrm{C}_{6} \mathrm{H}_{12} \\
\mathrm{O}\end{array}$ & Alcohol & $\begin{array}{c}903.9 \\
28, \\
1.703\end{array}$ \\
\hline 3 & $\begin{array}{l}111- \\
27-3\end{array}$ & 1-Hexanol & $\begin{array}{c}\mathrm{C}_{6} \mathrm{H}_{14} \\
\mathrm{O}\end{array}$ & Alcohol & $\begin{array}{c}915.9 \\
26, \\
1.518\end{array}$ \\
\hline 3 & $\begin{array}{c}1342 \\
9- \\
07-7\end{array}$ & 2-Propanol, 1-(2-methoxypropoxy)- & $\begin{array}{c}\mathrm{C}_{7} \mathrm{H}_{16} \\
\mathrm{O}_{3}\end{array}$ & Alcohol & $\begin{array}{c}1099 . \\
91, \\
1.544\end{array}$ \\
\hline 3 & $\begin{array}{c}76- \\
09-5\end{array}$ & 2,3-Butanediol, 2,3-dimethyl- & $\begin{array}{c}\mathrm{C}_{6} \mathrm{H}_{14} \\
\mathrm{O}_{2}\end{array}$ & Alcohol & $\begin{array}{c}1195 . \\
9, \\
1.782\end{array}$ \\
\hline 3 & $\begin{array}{c}0- \\
00-0\end{array}$ & Menthol, 1'-(butyn-3-one-1-yl)-, (1S,2S,5R)- & $\begin{array}{c}\mathrm{C}_{14} \\
\mathrm{H}_{22} \mathrm{O}_{2}\end{array}$ & Alcohol & $\begin{array}{c}1799 . \\
84, \\
2.244\end{array}$ \\
\hline 2 & $\begin{array}{c}71- \\
41-0\end{array}$ & 1-Pentanol & $\begin{array}{c}\mathrm{C}_{5} \mathrm{H}_{12} \\
\mathrm{O}\end{array}$ & Alcohol & $\begin{array}{c}771.9 \\
41, \\
1.663\end{array}$ \\
\hline 2 & $\begin{array}{c}1603 \\
480\end{array}$ & 1,3-Butanediol, (R)- & $\begin{array}{c}\mathrm{C}_{4} \mathrm{H}_{10} \\
\mathrm{O}_{2}\end{array}$ & Alcohol & $\begin{array}{c}823.9 \\
36, \\
1.214\end{array}$ \\
\hline 2 & $\begin{array}{c}2462 \\
1- \\
61-2\end{array}$ & 1,3-Butanediol, (S)- & $\begin{array}{c}\mathrm{C}_{4} \mathrm{H}_{10} \\
\mathrm{O}_{2}\end{array}$ & Alcohol & $\begin{array}{c}827.9 \\
35, \\
1.208\end{array}$ \\
\hline 2 & $\begin{array}{c}1489 \\
8- \\
79-4\end{array}$ & 2-Butanol, (R)- & $\begin{array}{c}\mathrm{C}_{4} \mathrm{H}_{10} \\
\mathrm{O}\end{array}$ & Alcohol & $\begin{array}{c}823.9 \\
36, \\
1.208\end{array}$ \\
\hline 2 & $\begin{array}{c}5595 \\
6- \\
37-1\end{array}$ & 2-Hepten-3-ol, 4,5-dimethyl- & $\begin{array}{c}\mathrm{C}_{9} \mathrm{H}_{18} \\
\mathrm{O}\end{array}$ & Alcohol & $\begin{array}{c}1543 . \\
86, \\
1.538\end{array}$ \\
\hline 2 & $\begin{array}{c}1872 \\
0- \\
66-6\end{array}$ & 3-Heptanol, 6-methyl- & $\begin{array}{c}\mathrm{C}_{8} \mathrm{H}_{18} \\
\mathrm{O}\end{array}$ & Alcohol & $\begin{array}{c}1079 . \\
91, \\
1.274\end{array}$ \\
\hline 2 & $\begin{array}{c}2443 \\
4- \\
07-9\end{array}$ & 4-Octyne-3,6-diol & $\begin{array}{c}\mathrm{C}_{8} \mathrm{H}_{14} \\
\mathrm{O}_{2}\end{array}$ & Alcohol & $\begin{array}{c}1219 . \\
9, \\
1.775\end{array}$ \\
\hline 1 & $\begin{array}{c}0- \\
00-0\end{array}$ & (2,3,3-Trimethyloxiranyl)methanol & $\begin{array}{c}\mathrm{C}_{6} \mathrm{H}_{12} \\
\mathrm{O}_{2}\end{array}$ & Alcohol & $\begin{array}{c}1083 . \\
91 \\
2.092\end{array}$ \\
\hline 1 & $\begin{array}{c}3643 \\
1- \\
59-1\end{array}$ & (5-Ethylcyclopent-1-enyl)methanol & $\begin{array}{c}\mathrm{C}_{8} \mathrm{H}_{14} \\
\mathrm{O}\end{array}$ & Alcohol & $\begin{array}{c}1071 . \\
91 \\
1.234\end{array}$ \\
\hline 1 & $\begin{array}{c}71- \\
36-3\end{array}$ & 1-Butanol & $\begin{array}{c}\mathrm{C}_{4} \mathrm{H}_{10} \\
\mathrm{O}\end{array}$ & Alcohol & $\begin{array}{c}595.9 \\
58\end{array}$ \\
\hline
\end{tabular}




\begin{tabular}{|c|c|c|c|c|c|}
\hline & & & & & 1.987 \\
\hline 1 & $\begin{array}{c}2790 \\
7- \\
00-2\end{array}$ & 1-Decyn-4-ol & $\begin{array}{c}\mathrm{C}_{10} \\
\mathrm{H}_{18} \mathrm{O}\end{array}$ & Alcohol & $\begin{array}{c}1131 . \\
9 \\
1.307\end{array}$ \\
\hline 1 & $\begin{array}{c}4798 \\
-44- \\
1\end{array}$ & 1-Hexen-3-ol & $\begin{array}{c}\mathrm{C}_{6} \mathrm{H}_{12} \\
\mathrm{O}\end{array}$ & Alcohol & $\begin{array}{c}1063 . \\
91 \\
1.360\end{array}$ \\
\hline 1 & $\begin{array}{c}3519 \\
2- \\
73-5\end{array}$ & 1-Nonen-4-ol & $\begin{array}{c}\mathrm{C}_{9} \mathrm{H}_{18} \\
\mathrm{O}\end{array}$ & Alcohol & $\begin{array}{c}1215 . \\
9, \\
1.947\end{array}$ \\
\hline 1 & $\begin{array}{c}7352 \\
73\end{array}$ & 1-Octanol, 2-butyl- & $\begin{array}{c}\mathrm{C}_{12} \\
\mathrm{H}_{26} \mathrm{O}\end{array}$ & Alcohol & $\begin{array}{c}1755 . \\
84, \\
1.122\end{array}$ \\
\hline 1 & $\begin{array}{c}4057 \\
5- \\
42-6\end{array}$ & 1-Octen-4-ol & $\begin{array}{c}\mathrm{C}_{8} \mathrm{H}_{16} \\
\mathrm{O}\end{array}$ & Alcohol & $\begin{array}{c}1203 . \\
9 \\
1.828\end{array}$ \\
\hline 1 & $\begin{array}{l}918- \\
85-4\end{array}$ & 1-Penten-3-ol, 3-methyl- & $\begin{array}{c}\mathrm{C}_{6} \mathrm{H}_{12} \\
\mathrm{O}\end{array}$ & Alcohol & $\begin{array}{c}899.9 \\
28, \\
1.043\end{array}$ \\
\hline 1 & $\begin{array}{l}112- \\
42-5\end{array}$ & 1-Undecanol & $\begin{array}{c}\mathrm{C}_{11} \\
\mathrm{H}_{24} \mathrm{O}\end{array}$ & Alcohol & $\begin{array}{c}1791 . \\
84, \\
1.815\end{array}$ \\
\hline 1 & $\begin{array}{c}2246 \\
0- \\
59-9\end{array}$ & 1,7-Octadien-3-ol, 2,6-dimethyl- & $\begin{array}{c}\mathrm{C}_{10} \\
\mathrm{H}_{18} \mathrm{O}\end{array}$ & Alcohol & $\begin{array}{c}1303 . \\
89 \\
1.399\end{array}$ \\
\hline 1 & $\begin{array}{c}5099 \\
7- \\
06-3\end{array}$ & 10-Methyl-10-nonadecanol & $\begin{array}{c}\mathrm{C}_{20} \\
\mathrm{H}_{42} \mathrm{O}\end{array}$ & Alcohol & $\begin{array}{c}1835 . \\
83 \\
1.300\end{array}$ \\
\hline 1 & $\begin{array}{c}1978 \\
0- \\
79-1\end{array}$ & 2-Hexyl-1-octanol & $\begin{array}{c}\mathrm{C}_{14} \\
\mathrm{H}_{30} \mathrm{O}\end{array}$ & Alcohol & $\begin{array}{c}2051 . \\
81, \\
1.379\end{array}$ \\
\hline 1 & $\begin{array}{c}3150 \\
2- \\
14-4\end{array}$ & 2-Nonen-1-ol, (E)- & $\begin{array}{c}\mathrm{C}_{9} \mathrm{H}_{18} \\
\mathrm{O}\end{array}$ & Alcohol & $\begin{array}{c}1367 . \\
88 \\
1.379\end{array}$ \\
\hline 1 & $\begin{array}{c}1867 \\
9- \\
48-6\end{array}$ & 2-Oxabicyclo[2.2.2]octan-6-ol, 1,3,3-trimethyl- & $\begin{array}{c}\mathrm{C}_{10} \\
\mathrm{H}_{18} \mathrm{O}_{2}\end{array}$ & Alcohol & $\begin{array}{c}1079 . \\
91 \\
1.241\end{array}$ \\
\hline 1 & $\begin{array}{c}1576 \\
-96- \\
1\end{array}$ & 2-Penten-1-ol, (E)- & $\begin{array}{c}\mathrm{C}_{5} \mathrm{H}_{10} \\
\mathrm{O}\end{array}$ & Alcohol & $\begin{array}{c}775.9 \\
4, \\
1.947\end{array}$ \\
\hline 1 & $\begin{array}{l}107- \\
98-2\end{array}$ & 2-Propanol, 1-methoxy- & $\begin{array}{c}\mathrm{C}_{4} \mathrm{H}_{10} \\
\mathrm{O}_{2}\end{array}$ & Alcohol & $\begin{array}{c}631.9 \\
55, \\
1.795\end{array}$ \\
\hline 1 & $\begin{array}{c}5817 \\
5- \\
57-8\end{array}$ & 2-Propyl-1-pentanol & $\begin{array}{c}\mathrm{C}_{8} \mathrm{H}_{18} \\
\mathrm{O}\end{array}$ & Alcohol & $\begin{array}{c}1387 . \\
88 \\
1.267\end{array}$ \\
\hline 1 & $\begin{array}{c}8206 \\
1- \\
20-9\end{array}$ & 2,3,4,5-Tetramethylcyclopent-2-en-1-ol & $\begin{array}{c}\mathrm{C}_{9} \mathrm{H}_{16} \\
\mathrm{O}\end{array}$ & Alcohol & $\begin{array}{c}1595 . \\
86, \\
1.769\end{array}$ \\
\hline 1 & $\begin{array}{c}8979 \\
4- \\
28-5\end{array}$ & 2,4-Dimethylcyclopentanol & $\begin{array}{c}\mathrm{C}_{7} \mathrm{H}_{14} \\
\mathrm{O}\end{array}$ & Alcohol & $\begin{array}{c}1071 . \\
91 \\
1.214\end{array}$ \\
\hline
\end{tabular}




\begin{tabular}{|c|c|c|c|c|c|}
\hline 1 & $\begin{array}{c}0- \\
00-0\end{array}$ & 2,4-Pentadien-1-ol, 3-ethyl-, (2Z)- & $\begin{array}{c}\mathrm{C}_{7} \mathrm{H}_{12} \\
\mathrm{O}\end{array}$ & Alcohol & $\begin{array}{c}1075 . \\
91, \\
1.360\end{array}$ \\
\hline 1 & $\begin{array}{c}0- \\
00-0\end{array}$ & 2,5-Dimethylhex-5-en-3-yn-2-ol & $\begin{array}{c}\mathrm{C}_{8} \mathrm{H}_{12} \\
\mathrm{O}\end{array}$ & Alcohol & $\begin{array}{c}947.9 \\
23, \\
1.115\end{array}$ \\
\hline 1 & $\begin{array}{l}627- \\
27-0\end{array}$ & 3-Buten-1-ol & $\begin{array}{c}\mathrm{C}_{4} \mathrm{H}_{8} \\
\mathrm{O}\end{array}$ & Alcohol & $\begin{array}{c}671.9 \\
51 \\
1.221\end{array}$ \\
\hline 1 & $\begin{array}{c}1872 \\
0- \\
62-2\end{array}$ & 3-Heptanol, 2-methyl- & $\begin{array}{c}\mathrm{C}_{8} \mathrm{H}_{18} \\
\mathrm{O}\end{array}$ & Alcohol & $\begin{array}{c}1203 . \\
9 \\
1.822\end{array}$ \\
\hline 1 & $\begin{array}{l}565- \\
67-3\end{array}$ & 3-Pentanol, 2-methyl- & $\begin{array}{c}\mathrm{C}_{6} \mathrm{H}_{14} \\
\mathrm{O}\end{array}$ & Alcohol & $\begin{array}{c}783.9 \\
4 \\
1.373\end{array}$ \\
\hline 1 & $\begin{array}{c}1460 \\
9- \\
79-1\end{array}$ & 3-Pentanol, 2,2,4,4-tetramethyl- & $\begin{array}{c}\mathrm{C}_{9} \mathrm{H}_{20} \\
\mathrm{O}\end{array}$ & Alcohol & $\begin{array}{c}671.9 \\
51, \\
1.287\end{array}$ \\
\hline 1 & $\begin{array}{c}3916 \\
1- \\
19-8\end{array}$ & 3-Penten-1-ol & $\begin{array}{c}\mathrm{C}_{5} \mathrm{H}_{10} \\
\mathrm{O}\end{array}$ & Alcohol & $\begin{array}{c}631.9 \\
55, \\
1.888\end{array}$ \\
\hline 1 & $\begin{array}{l}928- \\
92-7\end{array}$ & 4-Hexen-1-ol, (E)- & $\begin{array}{c}\mathrm{C}_{6} \mathrm{H}_{12} \\
\mathrm{O}\end{array}$ & Alcohol & $\begin{array}{c}903.9 \\
28, \\
1.696\end{array}$ \\
\hline 1 & $\begin{array}{l}623- \\
93-8\end{array}$ & 5-Nonanol & $\begin{array}{c}\mathrm{C}_{9} \mathrm{H}_{20} \\
\mathrm{O}\end{array}$ & Alcohol & $\begin{array}{c}1203 . \\
9 \\
1.822\end{array}$ \\
\hline 1 & $\begin{array}{c}2582 \\
6- \\
85-1\end{array}$ & $5 \cdot 7 \cdot \mathrm{H}, 10 \ddagger$-Eudesm-11-en- 1 -ol & $\begin{array}{c}\mathrm{C}_{15} \\
\mathrm{H}_{26} \mathrm{O}\end{array}$ & Alcohol & $\begin{array}{c}1935 . \\
82, \\
1.485\end{array}$ \\
\hline 1 & $\begin{array}{c}1192 \\
-78- \\
5\end{array}$ & 7-Oxabicyclo[4.1.0]heptan-2-ol & $\begin{array}{c}\mathrm{C}_{6} \mathrm{H}_{10} \\
\mathrm{O}_{2}\end{array}$ & Alcohol & $\begin{array}{c}1063 . \\
91 \\
1.353\end{array}$ \\
\hline 1 & $\begin{array}{c}3459 \\
0- \\
94-8\end{array}$ & Dipropylene glycol monomethyl ether & $\begin{array}{c}\mathrm{C}_{7} \mathrm{H}_{16} \\
\mathrm{O}_{3}\end{array}$ & Alcohol & $\begin{array}{c}1083 . \\
91 \\
1.445\end{array}$ \\
\hline 1 & $\begin{array}{l}110- \\
80-5\end{array}$ & Ethanol, 2-ethoxy- & $\begin{array}{c}\mathrm{C}_{4} \mathrm{H}_{10} \\
\mathrm{O}_{2}\end{array}$ & Alcohol & $\begin{array}{c}1111 . \\
91 \\
1.386\end{array}$ \\
\hline 1 & $\begin{array}{c}5353 \\
5- \\
33-4\end{array}$ & Heptanol & $\begin{array}{c}\mathrm{C}_{7} \mathrm{H}_{16} \\
\mathrm{O}\end{array}$ & Alcohol & $\begin{array}{c}1239 . \\
89 \\
1.525\end{array}$ \\
\hline 1 & $\begin{array}{l}764- \\
81-8\end{array}$ & Hydroperoxide, heptyl & $\begin{array}{c}\mathrm{C}_{7} \mathrm{H}_{16} \\
\mathrm{O}_{2}\end{array}$ & Alcohol & $\begin{array}{c}1083 . \\
91 \\
1.274\end{array}$ \\
\hline 1 & $\begin{array}{c}1139 \\
-08- \\
8\end{array}$ & Isolongifolan-8-ol & $\begin{array}{c}\mathrm{C}_{15} \\
\mathrm{H}_{26} \mathrm{O}\end{array}$ & Alcohol & $\begin{array}{c}1843 . \\
83 \\
1.412\end{array}$ \\
\hline 21 & $\begin{array}{c}66- \\
25-1\end{array}$ & Hexanal & $\begin{array}{c}\mathrm{C}_{6} \mathrm{H}_{12} \\
\mathrm{O}\end{array}$ & $\begin{array}{c}\text { Aldehy } \\
\text { de }\end{array}$ & $\begin{array}{c}831.9 \\
35, \\
1.241\end{array}$ \\
\hline 15 & 6789 & 3-Hexenal, (Z)- & $\mathrm{C}_{6} \mathrm{H}_{10}$ & Aldehy & 827.9 \\
\hline
\end{tabular}




\begin{tabular}{|c|c|c|c|c|c|}
\hline & $\begin{array}{c}-80- \\
6\end{array}$ & & $\mathrm{O}$ & de & $\begin{array}{c}35, \\
1.340\end{array}$ \\
\hline 7 & $\begin{array}{l}107- \\
02-8\end{array}$ & 2-Propenal & $\begin{array}{c}\mathrm{C}_{3} \mathrm{H}_{4} \\
\mathrm{O}\end{array}$ & $\begin{array}{c}\text { Aldehy } \\
\text { de }\end{array}$ & $\begin{array}{c}311.9 \\
87, \\
1.808\end{array}$ \\
\hline 7 & $\begin{array}{l}112- \\
31-2\end{array}$ & Decanal & $\begin{array}{c}\mathrm{C}_{10} \\
\mathrm{H}_{20} \mathrm{O}\end{array}$ & $\begin{array}{c}\text { Aldehy } \\
\text { de }\end{array}$ & $\begin{array}{c}1363 . \\
88, \\
1.399\end{array}$ \\
\hline 6 & $\begin{array}{l}645- \\
62-5\end{array}$ & 2-Hexenal, 2-ethyl- & $\begin{array}{c}\mathrm{C}_{8} \mathrm{H}_{14} \\
\mathrm{O}\end{array}$ & $\begin{array}{c}\text { Aldehy } \\
\text { de }\end{array}$ & $\begin{array}{c}959.9 \\
22, \\
1.096\end{array}$ \\
\hline 4 & $\begin{array}{c}1576 \\
-87- \\
0\end{array}$ & 2-Pentenal, (E)- & $\begin{array}{c}\mathrm{C}_{5} \mathrm{H}_{8} \\
\mathrm{O}\end{array}$ & $\begin{array}{c}\text { Aldehy } \\
\text { de }\end{array}$ & $\begin{array}{c}751.9 \\
43, \\
1.412\end{array}$ \\
\hline 4 & $\begin{array}{l}111- \\
71-7\end{array}$ & Heptanal & $\begin{array}{c}\mathrm{C}_{7} \mathrm{H}_{14} \\
\mathrm{O}\end{array}$ & $\begin{array}{c}\text { Aldehy } \\
\text { de }\end{array}$ & $\begin{array}{c}971.9 \\
21, \\
1.214\end{array}$ \\
\hline 4 & $\begin{array}{l}124- \\
19-6\end{array}$ & Nonanal & $\begin{array}{c}\mathrm{C}_{9} \mathrm{H}_{18} \\
\mathrm{O}\end{array}$ & $\begin{array}{c}\text { Aldehy } \\
\text { de }\end{array}$ & $\begin{array}{c}1219 . \\
9 \\
1.333\end{array}$ \\
\hline 3 & $\begin{array}{l}505- \\
57-7\end{array}$ & 2-Hexenal & $\begin{array}{c}\mathrm{C}_{6} \mathrm{H}_{10} \\
\mathrm{O}\end{array}$ & $\begin{array}{c}\text { Aldehy } \\
\text { de }\end{array}$ & $\begin{array}{c}915.9 \\
26, \\
1.353\end{array}$ \\
\hline 3 & $\begin{array}{c}8813 \\
95\end{array}$ & 2,4-Heptadienal, (E,E)- & $\begin{array}{c}\mathrm{C}_{7} \mathrm{H}_{10} \\
\mathrm{O}\end{array}$ & $\begin{array}{c}\text { Aldehy } \\
\text { de }\end{array}$ & $\begin{array}{c}1095 . \\
91, \\
1.485\end{array}$ \\
\hline 3 & $\begin{array}{l}142- \\
83-6\end{array}$ & 2,4-Hexadienal, (E,E)- & $\begin{array}{c}\mathrm{C}_{6} \mathrm{H}_{8} \\
\mathrm{O}\end{array}$ & $\begin{array}{c}\text { Aldehy } \\
\text { de }\end{array}$ & $\begin{array}{c}675.9 \\
5, \\
1.208\end{array}$ \\
\hline 3 & $\begin{array}{l}124- \\
13-0\end{array}$ & Octanal & $\begin{array}{c}\mathrm{C}_{8} \mathrm{H}_{16} \\
\mathrm{O}\end{array}$ & $\begin{array}{c}\text { Aldehy } \\
\text { de }\end{array}$ & $\begin{array}{c}1091 . \\
91, \\
1.214\end{array}$ \\
\hline 1 & $\begin{array}{c}0- \\
00-0\end{array}$ & 1,2,3-Trimethyl-cyclopent-2-enecarboxaldehyde & $\begin{array}{c}\mathrm{C}_{9} \mathrm{H}_{14} \\
\mathrm{O}\end{array}$ & $\begin{array}{c}\text { Aldehy } \\
\text { de }\end{array}$ & $\begin{array}{c}947.9 \\
23, \\
1.109\end{array}$ \\
\hline 1 & $\begin{array}{c}6728 \\
-26- \\
3\end{array}$ & 2-Hexenal, (E)- & $\begin{array}{c}\mathrm{C}_{6} \mathrm{H}_{10} \\
\mathrm{O}\end{array}$ & $\begin{array}{c}\text { Aldehy } \\
\text { de }\end{array}$ & $\begin{array}{c}915.9 \\
26, \\
1.353\end{array}$ \\
\hline 1 & $\begin{array}{c}2954 \\
8- \\
14-9\end{array}$ & 3-Cyclohexene-1-acetaldehyde, $\ddagger$,4-dimethyl- & $\begin{array}{c}\mathrm{C}_{10} \\
\mathrm{H}_{16} \mathrm{O}\end{array}$ & $\begin{array}{c}\text { Aldehy } \\
\text { de }\end{array}$ & $\begin{array}{c}1691 . \\
85, \\
1.287\end{array}$ \\
\hline 1 & $\begin{array}{c}4058 \\
-51- \\
9\end{array}$ & 3,4-Pentadienal, 2,2-dimethyl- & $\begin{array}{c}\mathrm{C}_{7} \mathrm{H}_{10} \\
\mathrm{O}\end{array}$ & $\begin{array}{c}\text { Aldehy } \\
\text { de }\end{array}$ & $\begin{array}{c}1083 . \\
91 \\
1.030\end{array}$ \\
\hline 1 & $\begin{array}{c}5204 \\
-80- \\
8\end{array}$ & 4-Pentenal, 2-ethyl- & $\begin{array}{c}\mathrm{C}_{7} \mathrm{H}_{12} \\
\mathrm{O}\end{array}$ & $\begin{array}{c}\text { Aldehy } \\
\text { de }\end{array}$ & $\begin{array}{c}1071 . \\
91 \\
1.016\end{array}$ \\
\hline 1 & $\begin{array}{c}97- \\
96-1\end{array}$ & Butanal, 2-ethyl- & $\begin{array}{c}\mathrm{C}_{6} \mathrm{H}_{12} \\
\mathrm{O}\end{array}$ & $\begin{array}{c}\text { Aldehy } \\
\text { de }\end{array}$ & $\begin{array}{c}1071 . \\
91, \\
1.267\end{array}$ \\
\hline 1 & $\begin{array}{l}112- \\
54-9 \\
\end{array}$ & Dodecanal & $\begin{array}{c}\mathrm{C}_{12} \\
\mathrm{H}_{42} \mathrm{O} \\
\end{array}$ & $\begin{array}{c}\text { Aldehy } \\
\text { de }\end{array}$ & $\begin{array}{c}1687 . \\
85,\end{array}$ \\
\hline
\end{tabular}




\begin{tabular}{|c|c|c|c|c|c|}
\hline & & & & & 1.472 \\
\hline 1 & $\begin{array}{l}123- \\
05-7\end{array}$ & Hexanal, 2-ethyl- & $\begin{array}{c}\mathrm{C}_{8} \mathrm{H}_{16} \\
\mathrm{O}\end{array}$ & $\begin{array}{c}\text { Aldehy } \\
\text { de }\end{array}$ & $\begin{array}{c}1043 . \\
91 \\
1.135\end{array}$ \\
\hline 1 & $\begin{array}{c}78- \\
85-3\end{array}$ & Methacrolein & $\begin{array}{c}\mathrm{C}_{4} \mathrm{H}_{6} \\
\mathrm{O}\end{array}$ & $\begin{array}{c}\text { Aldehy } \\
\text { de }\end{array}$ & $\begin{array}{c}1075 . \\
91, \\
1.327\end{array}$ \\
\hline 1 & $\begin{array}{c}3162 \\
49\end{array}$ & Pentadecanal- & $\begin{array}{c}\mathrm{C}_{15} \\
\mathrm{H}_{30} \mathrm{O}\end{array}$ & $\begin{array}{c}\text { Aldehy } \\
\text { de }\end{array}$ & $\begin{array}{c}2375 . \\
78, \\
1.756\end{array}$ \\
\hline 1 & $\begin{array}{l}123- \\
38-6\end{array}$ & Propanal & $\begin{array}{c}\mathrm{C}_{3} \mathrm{H}_{6} \\
\mathrm{O}\end{array}$ & $\begin{array}{c}\text { Aldehy } \\
\text { de }\end{array}$ & $\begin{array}{c}319.9 \\
86, \\
1.478\end{array}$ \\
\hline 22 & $\begin{array}{l}109- \\
66-0\end{array}$ & Pentane & $\mathrm{C}_{5} \mathrm{H}_{12}$ & Alkane & $\begin{array}{c}327.9 \\
85, \\
1.030\end{array}$ \\
\hline 10 & $\begin{array}{l}124- \\
18-5\end{array}$ & Decane & $\begin{array}{l}\mathrm{C}_{10} \\
\mathrm{H}_{22}\end{array}$ & Alkane & $\begin{array}{c}1227 . \\
9 \\
1.023\end{array}$ \\
\hline 8 & $\begin{array}{l}111- \\
84-2\end{array}$ & Nonane & $\mathrm{C}_{9} \mathrm{H}_{20}$ & Alkane & $\begin{array}{c}963.9 \\
22, \\
0.970\end{array}$ \\
\hline 6 & $\begin{array}{l}629- \\
59-4\end{array}$ & Tetradecane & $\begin{array}{l}\mathrm{C}_{14} \\
\mathrm{H}_{30}\end{array}$ & Alkane & $\begin{array}{c}1651 . \\
85 \\
1.109\end{array}$ \\
\hline 5 & $\begin{array}{c}1730 \\
1- \\
23-4\end{array}$ & Undecane, 2,6-dimethyl- & $\begin{array}{l}\mathrm{C}_{13} \\
\mathrm{H}_{28}\end{array}$ & Alkane & $\begin{array}{c}1363 . \\
88 \\
1.049\end{array}$ \\
\hline 4 & $\begin{array}{c}5410 \\
5- \\
67-8\end{array}$ & Heptadecane, 2,6-dimethyl- & $\begin{array}{l}\mathrm{C}_{19} \\
\mathrm{H}_{40}\end{array}$ & Alkane & $\begin{array}{c}2235 . \\
79 \\
1.181\end{array}$ \\
\hline 4 & $\begin{array}{l}544- \\
76-3\end{array}$ & Hexadecane & $\begin{array}{l}\mathrm{C}_{16} \\
\mathrm{H}_{34}\end{array}$ & Alkane & $\begin{array}{c}2147 . \\
8, \\
1.162\end{array}$ \\
\hline 3 & $\begin{array}{c}6210 \\
8- \\
22-9\end{array}$ & Decane, 2,5,9-trimethyl- & $\begin{array}{l}\mathrm{C}_{13} \\
\mathrm{H}_{28}\end{array}$ & Alkane & $\begin{array}{c}1311 . \\
89 \\
1.030\end{array}$ \\
\hline 3 & $\begin{array}{c}7464 \\
5- \\
98-0\end{array}$ & Dodecane, 2,7,10-trimethyl- & $\begin{array}{l}\mathrm{C}_{15} \\
\mathrm{H}_{32}\end{array}$ & Alkane & $\begin{array}{c}2163 . \\
8, \\
1.135\end{array}$ \\
\hline 3 & $\begin{array}{c}5504 \\
5- \\
14-2\end{array}$ & Tetradecane, 4-ethyl- & $\begin{array}{l}\mathrm{C}_{16} \\
\mathrm{H}_{34}\end{array}$ & Alkane & $\begin{array}{c}2231 . \\
79 \\
1.181\end{array}$ \\
\hline 2 & $\begin{array}{c}6210 \\
8- \\
25-2\end{array}$ & Decane, 2,6,7-trimethyl- & $\begin{array}{l}\mathrm{C}_{13} \\
\mathrm{H}_{28}\end{array}$ & Alkane & $\begin{array}{c}1455 . \\
87 \\
1.056\end{array}$ \\
\hline 2 & $\begin{array}{c}1560 \\
-97- \\
0\end{array}$ & Dodecane, 2-methyl- & $\begin{array}{l}\mathrm{C}_{13} \\
\mathrm{H}_{28}\end{array}$ & Alkane & $\begin{array}{c}1655 . \\
85 \\
1.102\end{array}$ \\
\hline 2 & $\begin{array}{c}3891 \\
-98- \\
3\end{array}$ & Dodecane, 2,6,10-trimethyl- & $\begin{array}{l}\mathrm{C}_{15} \\
\mathrm{H}_{32}\end{array}$ & Alkane & $\begin{array}{c}1623 . \\
86 \\
1.082\end{array}$ \\
\hline
\end{tabular}




\begin{tabular}{|c|c|c|c|c|c|}
\hline 2 & $\begin{array}{l}496- \\
11-7\end{array}$ & Indane & $\mathrm{C}_{9} \mathrm{H}_{10}$ & Alkane & $\begin{array}{c}1163 . \\
9, \\
1.399\end{array}$ \\
\hline 2 & $\begin{array}{l}629- \\
92-5\end{array}$ & Nonadecane & $\begin{array}{l}\mathrm{C}_{19} \\
\mathrm{H}_{40}\end{array}$ & Alkane & $\begin{array}{c}1827 . \\
84, \\
1.082\end{array}$ \\
\hline 2 & $\begin{array}{c}1054 \\
4- \\
96-4\end{array}$ & Octadecane, 6-methyl- & $\begin{array}{l}\mathrm{C}_{19} \\
\mathrm{H}_{40}\end{array}$ & Alkane & $\begin{array}{c}2227 . \\
8, \\
1.168\end{array}$ \\
\hline 2 & $\begin{array}{c}1586 \\
9- \\
86-0\end{array}$ & Octane, 4-ethyl- & $\begin{array}{l}\mathrm{C}_{10} \\
\mathrm{H}_{22}\end{array}$ & Alkane & $\begin{array}{c}1279 . \\
89 \\
1.043\end{array}$ \\
\hline 1 & $\begin{array}{c}6975 \\
-98- \\
0\end{array}$ & Decane, 2-methyl- & $\begin{array}{l}\mathrm{C}_{11} \\
\mathrm{H}_{42}\end{array}$ & Alkane & $\begin{array}{c}1895 . \\
83, \\
1.122\end{array}$ \\
\hline 1 & $\begin{array}{c}6210 \\
8- \\
23-0\end{array}$ & Decane, 2,5,6-trimethyl- & $\begin{array}{l}\mathrm{C}_{13} \\
\mathrm{H}_{28}\end{array}$ & Alkane & $\begin{array}{c}1107 . \\
91, \\
0.983\end{array}$ \\
\hline 1 & $\begin{array}{c}6210 \\
8- \\
26-3\end{array}$ & Decane, 2,6,8-trimethyl- & $\begin{array}{l}\mathrm{C}_{13} \\
\mathrm{H}_{28}\end{array}$ & Alkane & $\begin{array}{c}1631 . \\
85 \\
1.089\end{array}$ \\
\hline 1 & $\begin{array}{c}1731 \\
2- \\
54-8\end{array}$ & Decane, 3,7-dimethyl- & $\begin{array}{l}\mathrm{C}_{12} \\
\mathrm{H}_{26}\end{array}$ & Alkane & $\begin{array}{c}1243 . \\
89 \\
1.030\end{array}$ \\
\hline 1 & $\begin{array}{c}2847 \\
-72- \\
5\end{array}$ & Decane, 4-methyl- & $\begin{array}{l}\mathrm{C}_{11} \\
\mathrm{H}_{42}\end{array}$ & Alkane & $\begin{array}{c}1367 . \\
88 \\
1.036\end{array}$ \\
\hline 1 & $\begin{array}{c}6044 \\
-71- \\
9\end{array}$ & Dodecane, 6-methyl- & $\begin{array}{l}\mathrm{C}_{13} \\
\mathrm{H}_{28}\end{array}$ & Alkane & $\begin{array}{c}1279 . \\
89 \\
1.023\end{array}$ \\
\hline 1 & $\begin{array}{l}629- \\
78-7\end{array}$ & Heptadecane & $\begin{array}{l}\mathrm{C}_{17} \\
\mathrm{H}_{36}\end{array}$ & Alkane & $\begin{array}{c}2147 . \\
8, \\
1.155\end{array}$ \\
\hline 1 & $\begin{array}{c}1560 \\
-89- \\
0\end{array}$ & Heptadecane, 2-methyl- & $\begin{array}{l}\mathrm{C}_{18} \\
\mathrm{H}_{38}\end{array}$ & Alkane & $\begin{array}{c}1923 . \\
83 \\
1.129\end{array}$ \\
\hline 1 & $\begin{array}{c}1834 \\
4- \\
37-1\end{array}$ & Heptadecane, 2,6,10,14-tetramethyl- & $\begin{array}{l}\mathrm{C}_{21} \\
\mathrm{H}_{44}\end{array}$ & Alkane & $\begin{array}{c}1759 . \\
84, \\
1.102\end{array}$ \\
\hline 1 & $\begin{array}{l}142- \\
82-5\end{array}$ & Heptane & $\mathrm{C}_{7} \mathrm{H}_{16}$ & Alkane & $\begin{array}{c}671.9 \\
51, \\
0.983\end{array}$ \\
\hline 1 & $\begin{array}{c}1072 \\
-05- \\
5\end{array}$ & Heptane, 2,6-dimethyl- & $\mathrm{C}_{9} \mathrm{H}_{20}$ & Alkane & $\begin{array}{c}1071 . \\
91 \\
1.181\end{array}$ \\
\hline 1 & $\begin{array}{c}1586 \\
9- \\
80-4\end{array}$ & Heptane, 3-ethyl- & $\mathrm{C}_{9} \mathrm{H}_{20}$ & Alkane & $\begin{array}{c}1279 . \\
89 \\
1.030\end{array}$ \\
\hline 1 & $\begin{array}{c}0- \\
00-0\end{array}$ & Heptane, 3-ethyl-5-methylene- & $\begin{array}{l}\mathrm{C}_{10} \\
\mathrm{H}_{20}\end{array}$ & Alkane & $\begin{array}{c}1067 . \\
91, \\
1.228\end{array}$ \\
\hline 1 & 1632 & Heptane, 3-methylene- & $\mathrm{C}_{8} \mathrm{H}_{16}$ & Alkane & 1083. \\
\hline
\end{tabular}




\begin{tabular}{|c|c|c|c|c|c|}
\hline & $-16-$ & & & & 91, \\
\hline & 2 & & & & 1.267 \\
\hline 1 & $\begin{array}{l}638- \\
36-8\end{array}$ & Hexadecane, 2,6,10,14-tetramethyl- & $\begin{array}{l}\mathrm{C}_{20} \\
\mathrm{H}_{42}\end{array}$ & Alkane & $\begin{array}{c}2347 . \\
78, \\
1.195\end{array}$ \\
\hline 1 & $\begin{array}{c}6418 \\
-43- \\
5\end{array}$ & Hexadecane, 3-methyl- & $\begin{array}{c}\mathrm{C}_{17} \mathrm{H}_{3} \\
6\end{array}$ & Alkane & $\begin{array}{c}2103 . \\
81 \\
1.148\end{array}$ \\
\hline 1 & $\begin{array}{l}590- \\
73-8\end{array}$ & Hexane, 2,2-dimethyl- & $\mathrm{C}_{8} \mathrm{H}_{18}$ & Alkane & $\begin{array}{c}2219 . \\
8, \\
1.168\end{array}$ \\
\hline 1 & $\begin{array}{l}589- \\
34-4\end{array}$ & Hexane, 3-methyl- & $\mathrm{C}_{7} \mathrm{H}_{16}$ & Alkane & $\begin{array}{c}671.9 \\
51, \\
0.983\end{array}$ \\
\hline 1 & $\begin{array}{c}75- \\
28-5\end{array}$ & Isobutane & $\mathrm{C}_{4} \mathrm{H}_{10}$ & Alkane & $\begin{array}{c}1071 . \\
91 \\
1.155\end{array}$ \\
\hline 1 & $\begin{array}{c}3590 \\
71\end{array}$ & n-Nonylcyclohexane & $\begin{array}{l}\mathrm{C}_{15} \\
\mathrm{H}_{30}\end{array}$ & Alkane & $\begin{array}{c}1935 . \\
82 \\
1.221\end{array}$ \\
\hline 1 & $\begin{array}{l}463- \\
82-1\end{array}$ & Neopentane & $\mathrm{C}_{5} \mathrm{H}_{12}$ & Alkane & $\begin{array}{c}1479 . \\
87 \\
1.478\end{array}$ \\
\hline 1 & $\begin{array}{c}3221 \\
-61- \\
2\end{array}$ & Octane, 2-methyl- & $\mathrm{C}_{9} \mathrm{H}_{20}$ & Alkane & $\begin{array}{c}2235 . \\
79 \\
1.181\end{array}$ \\
\hline 1 & $\begin{array}{c}1636 \\
-41- \\
5\end{array}$ & Octane, 4,5-diethyl- & $\begin{array}{l}\mathrm{C}_{12} \\
\mathrm{H}_{26}\end{array}$ & Alkane & $\begin{array}{c}911.9 \\
27, \\
0.970\end{array}$ \\
\hline 1 & $\begin{array}{l}629- \\
62-9\end{array}$ & Pentadecane & $\begin{array}{c}\mathrm{C}_{15} \mathrm{H}_{3} \\
2\end{array}$ & Alkane & $\begin{array}{c}1815 . \\
84, \\
1.122\end{array}$ \\
\hline 1 & $\begin{array}{c}3892 \\
-00- \\
0\end{array}$ & Pentadecane, 2,6,10-trimethyl- & $\begin{array}{c}\mathrm{C}_{18} \mathrm{H}_{3} \\
8\end{array}$ & Alkane & $\begin{array}{c}2067 . \\
81, \\
1.129\end{array}$ \\
\hline 1 & $\begin{array}{c}2882 \\
-96- \\
4\end{array}$ & Pentadecane, 3-methyl- & $\begin{array}{c}\mathrm{C}_{16} \mathrm{H}_{3} \\
4\end{array}$ & Alkane & $\begin{array}{c}1939 . \\
82 \\
1.135\end{array}$ \\
\hline 1 & $\begin{array}{l}590- \\
35-2\end{array}$ & Pentane, 2,2-dimethyl- & $\mathrm{C}_{7} \mathrm{H}_{16}$ & Alkane & $\begin{array}{c}539.9 \\
64, \\
1.003\end{array}$ \\
\hline 1 & $\begin{array}{l}108- \\
08-7\end{array}$ & Pentane, 2,4-dimethyl- & $\mathrm{C}_{7} \mathrm{H}_{16}$ & Alkane & $\begin{array}{c}547.9 \\
63, \\
1.003\end{array}$ \\
\hline 1 & $\begin{array}{c}1907 \\
4- \\
25-0\end{array}$ & Tricyclo[3.2.1.0(1,5)]octane & $\mathrm{C}_{8} \mathrm{H}_{12}$ & Alkane & $\begin{array}{c}935.9 \\
24, \\
1.109\end{array}$ \\
\hline 1 & $\begin{array}{c}7045 \\
-71- \\
8\end{array}$ & Undecane, 2-methyl- & $\begin{array}{l}\mathrm{C}_{12} \\
\mathrm{H}_{26}\end{array}$ & Alkane & $\begin{array}{c}2091 . \\
81, \\
1.142\end{array}$ \\
\hline 12 & $\begin{array}{l}591- \\
93-5\end{array}$ & 1,4-Pentadiene & $\mathrm{C}_{5} \mathrm{H}_{8}$ & Alkene & $\begin{array}{c}375.9 \\
8,\end{array}$ \\
\hline
\end{tabular}




\begin{tabular}{|c|c|c|c|c|c|}
\hline & & & & & 1.129 \\
\hline \multirow{4}{*}{10} & 4008 & & & & 943.9 \\
\hline & 7- & 1,3-trans,5-cis-octatriene & $\mathrm{C}_{8} \mathrm{H}_{12}$ & Alkene & 24 \\
\hline & $61-4$ & & & & 1.135 \\
\hline & 1002 & & & & 867.9 \\
\hline 9 & $\begin{array}{c}-33- \\
1\end{array}$ & 1,3-Octadiene & $\mathrm{C}_{8} \mathrm{H}_{14}$ & Alkene & $\begin{array}{c}31, \\
1.056\end{array}$ \\
\hline \multirow[t]{2}{*}{4} & $\begin{array}{l}592- \\
76-7\end{array}$ & 1-Heptene & $\mathrm{C}_{7} \mathrm{H}_{14}$ & Alkene & $\begin{array}{c}655.9 \\
52, \\
1.010\end{array}$ \\
\hline & 2645 & & & & 1051. \\
\hline 4 & $\begin{array}{c}6- \\
76-8\end{array}$ & 2-Hexene, 3,5,5-trimethyl- & $\mathrm{C}_{9} \mathrm{H}_{18}$ & Alkene & $\begin{array}{c}91 \\
1.511\end{array}$ \\
\hline 4 & $\begin{array}{l}646- \\
04-8\end{array}$ & 2-Pentene, (E)- & $\mathrm{C}_{5} \mathrm{H}_{10}$ & Alkene & $\begin{array}{c}339.9 \\
84, \\
1.056\end{array}$ \\
\hline \multirow[t]{2}{*}{4} & $\begin{array}{c}1647 \\
-08- \\
1\end{array}$ & 4,4-Dimethyl-1-hexene & $\mathrm{C}_{8} \mathrm{H}_{16}$ & Alkene & $\begin{array}{c}1075 . \\
91 \\
1.280\end{array}$ \\
\hline & 1574 & & & & 391.9 \\
\hline \multirow[t]{2}{*}{3} & $\begin{array}{c}-41- \\
0\end{array}$ & 1,3-Pentadiene, (Z)- & $\mathrm{C}_{5} \mathrm{H}_{8}$ & Alkene & $\begin{array}{c}79 \\
1.129\end{array}$ \\
\hline & 5675 & & & & 663.9 \\
\hline 3 & $\begin{array}{c}-22- \\
9\end{array}$ & 1,4-Heptadiene & $\mathrm{C}_{7} \mathrm{H}_{12}$ & Alkene & $\begin{array}{c}52 \\
1.049\end{array}$ \\
\hline \multirow[t]{2}{*}{3} & $\begin{array}{l}111- \\
67-1\end{array}$ & 2-Octene & $\mathrm{C}_{8} \mathrm{H}_{16}$ & Alkene & $\begin{array}{c}815.9 \\
36, \\
1.016\end{array}$ \\
\hline & 1663 & & & & 943.9 \\
\hline 3 & $\begin{array}{c}1- \\
66-6\end{array}$ & E,Z-4-Ethylidenecyclohexene & $\mathrm{C}_{8} \mathrm{H}_{12}$ & Alkene & $\begin{array}{c}24, \\
1.135\end{array}$ \\
\hline \multirow[t]{2}{*}{2} & $\begin{array}{l}563- \\
45-1\end{array}$ & 1-Butene, 3-methyl- & $\mathrm{C}_{5} \mathrm{H}_{10}$ & Alkene & $\begin{array}{c}339.9 \\
84, \\
1.049\end{array}$ \\
\hline & 1984 & & & & 943.9 \\
\hline \multirow[t]{2}{*}{2} & $\begin{array}{c}1- \\
74-8\end{array}$ & 1-Ethyl-1,4-cyclohexadiene & $\mathrm{C}_{8} \mathrm{H}_{12}$ & Alkene & $\begin{array}{c}24, \\
1.129\end{array}$ \\
\hline & 7116 & & & & 2007. \\
\hline \multirow[t]{2}{*}{2} & $\begin{array}{c}-86- \\
1\end{array}$ & 1-Hexene, 5,5-dimethyl- & $\mathrm{C}_{8} \mathrm{H}_{16}$ & Alkene & $\begin{array}{c}82 \\
1.261\end{array}$ \\
\hline & 6537 & & & & 919.9 \\
\hline 2 & $\begin{array}{c}8- \\
76-9\end{array}$ & 1,2,4,4-Tetramethylcyclopentene & $\mathrm{C}_{9} \mathrm{H}_{16}$ & Alkene & $\begin{array}{c}26, \\
1.010\end{array}$ \\
\hline 2 & $\begin{array}{l}504- \\
60-9\end{array}$ & 1,3-Pentadiene & $\mathrm{C}_{5} \mathrm{H}_{8}$ & Alkene & $\begin{array}{c}375.9 \\
8, \\
1.122\end{array}$ \\
\hline 2 & $\begin{array}{l}563- \\
46-2\end{array}$ & 2-Methyl-1-butene & $\mathrm{C}_{5} \mathrm{H}_{10}$ & Alkene & $\begin{array}{c}351.9 \\
83, \\
1.056\end{array}$ \\
\hline 1 & $\begin{array}{c}5672 \\
8- \\
10-0 \\
\end{array}$ & 1-Hexene, 3,4,5-trimethyl- & $\mathrm{C}_{9} \mathrm{H}_{18}$ & Alkene & $\begin{array}{c}1543 . \\
86, \\
1.518\end{array}$ \\
\hline
\end{tabular}




\begin{tabular}{|c|c|c|c|c|c|}
\hline 1 & $\begin{array}{l}124- \\
11-8\end{array}$ & 1-Nonene & $\mathrm{C}_{9} \mathrm{H}_{18}$ & Alkene & $\begin{array}{c}951.9 \\
23, \\
1.003\end{array}$ \\
\hline 1 & $\begin{array}{c}2437 \\
-56- \\
1\end{array}$ & 1-Tridecene & $\begin{array}{l}\mathrm{C}_{13} \\
\mathrm{H}_{26}\end{array}$ & Alkene & $\begin{array}{c}1639 . \\
85 \\
1.155\end{array}$ \\
\hline 1 & $\begin{array}{c}7463 \\
0- \\
39-0\end{array}$ & 1-Undecene, 4-methyl- & $\begin{array}{l}\mathrm{C}_{12} \\
\mathrm{H}_{24}\end{array}$ & Alkene & $\begin{array}{c}1755 . \\
84, \\
1.115\end{array}$ \\
\hline 1 & $\begin{array}{c}2196 \\
4- \\
49-8 \\
2203\end{array}$ & 1,13-Tetradecadiene & $\begin{array}{l}\mathrm{C}_{14} \\
\mathrm{H}_{26}\end{array}$ & Alkene & $\begin{array}{c}2111 . \\
81 \\
1.241 \\
947.9\end{array}$ \\
\hline 1 & $\begin{array}{c}8- \\
69-3\end{array}$ & 1,3,6-Octatriene, (E,E)- & $\mathrm{C}_{8} \mathrm{H}_{12}$ & Alkene & $\begin{array}{c}23 \\
1.122\end{array}$ \\
\hline 1 & $\begin{array}{c}0- \\
00-0\end{array}$ & 1,3(Z),13-Tetradecatriene & $\begin{array}{l}\mathrm{C}_{14} \\
\mathrm{H}_{24}\end{array}$ & Alkene & $\begin{array}{c}1935 . \\
82, \\
1.294\end{array}$ \\
\hline 1 & $\begin{array}{c}1364 \\
3- \\
06-6\end{array}$ & 1,6-Heptadiene, 2-methyl- & $\mathrm{C}_{8} \mathrm{H}_{14}$ & Alkene & $\begin{array}{c}1099 . \\
91 \\
1.076\end{array}$ \\
\hline 1 & $\begin{array}{c}2471 \\
-83- \\
2\end{array}$ & 1H-Indene, 1-ethylidene- & $\begin{array}{l}\mathrm{C}_{11} \\
\mathrm{H}_{10}\end{array}$ & Alkene & $\begin{array}{c}1615 . \\
86, \\
2.290\end{array}$ \\
\hline 1 & $\begin{array}{l}627- \\
20-3\end{array}$ & 2-Pentene, (Z)- & $\mathrm{C}_{5} \mathrm{H}_{10}$ & Alkene & $\begin{array}{c}343.9 \\
84, \\
1.049\end{array}$ \\
\hline 1 & $\begin{array}{l}598- \\
96-9\end{array}$ & 2-Pentene, 3,4,4-trimethyl- & $\mathrm{C}_{8} \mathrm{H}_{16}$ & Alkene & $\begin{array}{c}1131 . \\
9 \\
1.300\end{array}$ \\
\hline 1 & $\begin{array}{c}7476 \\
4- \\
46-8\end{array}$ & 3-Heptene, 3-ethyl- & $\mathrm{C}_{9} \mathrm{H}_{18}$ & Alkene & $\begin{array}{c}959.9 \\
22, \\
1.082\end{array}$ \\
\hline 1 & $\begin{array}{c}4144 \\
6- \\
67-7\end{array}$ & 3-Tetradecene, (Z)- & $\begin{array}{l}\mathrm{C}_{14} \\
\mathrm{H}_{28}\end{array}$ & Alkene & $\begin{array}{c}1639 . \\
85 \\
1.155\end{array}$ \\
\hline 1 & $\begin{array}{c}4144 \\
6- \\
53-1\end{array}$ & 3-Tridecene, (Z)- & $\begin{array}{l}\mathrm{C}_{13} \\
\mathrm{H}_{26}\end{array}$ & Alkene & $\begin{array}{c}1643 . \\
85, \\
1.142\end{array}$ \\
\hline 1 & $\begin{array}{c}3705 \\
0- \\
04-7\end{array}$ & 3,4-Decadiene & $\begin{array}{l}\mathrm{C}_{10} \\
\mathrm{H}_{18}\end{array}$ & Alkene & $\begin{array}{c}1423 . \\
88 \\
1.360\end{array}$ \\
\hline 1 & $\begin{array}{c}4144 \\
6- \\
78-0\end{array}$ & 4-Tetradecene, (E)- & $\begin{array}{l}\mathrm{C}_{14} \\
\mathrm{H}_{28}\end{array}$ & Alkene & $\begin{array}{c}2119 . \\
81 \\
1.188\end{array}$ \\
\hline 1 & $\begin{array}{c}3550 \\
7- \\
09-6\end{array}$ & 7-Hexadecene, (Z)- & $\begin{array}{l}\mathrm{C}_{16} \\
\mathrm{H}_{32}\end{array}$ & Alkene & $\begin{array}{c}2115 . \\
81 \\
1.208\end{array}$ \\
\hline 1 & $\begin{array}{c}5429 \\
0- \\
12-9\end{array}$ & 8-Heptadecene & $\begin{array}{l}\mathrm{C}_{17} \\
\mathrm{H}_{34}\end{array}$ & Alkene & $\begin{array}{c}2147 . \\
8, \\
1.208\end{array}$ \\
\hline 1 & 3404 & Unknown 3 & $\mathrm{C}_{7} \mathrm{H}_{14}$ & Alkene & 679.9 \\
\hline
\end{tabular}




\begin{tabular}{|c|c|c|c|c|c|}
\hline & $\begin{array}{c}-62- \\
4\end{array}$ & & & & $\begin{array}{c}5, \\
1.267\end{array}$ \\
\hline & 2461 & & & & 935.9 \\
\hline 2 & $\begin{array}{c}2- \\
83-7\end{array}$ & 1-Octen-4-yne & $\mathrm{C}_{8} \mathrm{H}_{12}$ & Alkyne & $\begin{array}{c}24, \\
1.129\end{array}$ \\
\hline 1 & $\begin{array}{c}7600 \\
3- \\
40-2\end{array}$ & 1-Hepten-5-yne, 2-methyl-3-methylene- & $\mathrm{C}_{9} \mathrm{H}_{12}$ & Alkyne & $\begin{array}{c}1087 . \\
91, \\
1.195\end{array}$ \\
\hline 1 & $\begin{array}{l}821- \\
08-9\end{array}$ & 1,5-Hexadien-3-yne & $\mathrm{C}_{6} \mathrm{H}_{6}$ & Alkyne & $\begin{array}{c}675.9 \\
5, \\
1.208\end{array}$ \\
\hline 1 & $\begin{array}{c}1064 \\
45- \\
95-8 \\
4189\end{array}$ & 2-Octen-4-yne, (Z)- & $\mathrm{C}_{8} \mathrm{H}_{12}$ & Alkyne & $\begin{array}{c}1015 . \\
92, \\
1.148 \\
1087 .\end{array}$ \\
\hline 1 & $\begin{array}{c}4189 \\
8- \\
89-9\end{array}$ & 2,3-Heptadien-5-yne, 2,4-dimethyl- & $\mathrm{C}_{9} \mathrm{H}_{12}$ & Alkyne & $\begin{array}{c}1087 . \\
91 \\
1.214\end{array}$ \\
\hline 6 & $\begin{array}{l}123- \\
62-6\end{array}$ & Propanoic acid, anhydride & $\begin{array}{c}\mathrm{C}_{6} \mathrm{H}_{10} \\
\mathrm{O}_{3}\end{array}$ & $\begin{array}{c}\text { Anhydr } \\
\text { ide }\end{array}$ & $\begin{array}{c}635.9 \\
54, \\
1.894\end{array}$ \\
\hline 1 & $\begin{array}{c}1538 \\
-75- \\
6\end{array}$ & 2,2-Dimethylpropanoic anhydride & $\begin{array}{c}\mathrm{C}_{10} \\
\mathrm{H}_{18} \mathrm{O}_{3}\end{array}$ & $\begin{array}{c}\text { Anhydr } \\
\text { ide }\end{array}$ & $\begin{array}{c}1067 . \\
91, \\
1.214\end{array}$ \\
\hline 1 & $\begin{array}{l}106- \\
31-0\end{array}$ & Butanoic acid, anhydride & $\begin{array}{c}\mathrm{C}_{8} \mathrm{H}_{14} \\
\mathrm{O}_{3}\end{array}$ & $\begin{array}{c}\text { Anhydr } \\
\text { ide }\end{array}$ & $\begin{array}{c}1543 . \\
86, \\
1.525\end{array}$ \\
\hline 1 & $\begin{array}{c}97- \\
72-3\end{array}$ & Propanoic acid, 2-methyl-, anhydride & $\begin{array}{c}\mathrm{C}_{8} \mathrm{H}_{14} \\
\mathrm{O}_{3}\end{array}$ & $\begin{array}{c}\text { Anhydr } \\
\text { ide }\end{array}$ & $\begin{array}{c}1543 . \\
86, \\
1.518\end{array}$ \\
\hline 9 & $\begin{array}{c}4537 \\
-15- \\
9\end{array}$ & Benzene, (1-butylheptyl)- & $\begin{array}{l}\mathrm{C}_{17} \\
\mathrm{H}_{28}\end{array}$ & $\begin{array}{l}\text { Aromat } \\
\text { ic }\end{array}$ & $\begin{array}{c}2059 . \\
81 \\
1.399\end{array}$ \\
\hline 7 & $\begin{array}{c}2719 \\
-62- \\
2\end{array}$ & Benzene, (1-pentylheptyl)- & $\begin{array}{l}\mathrm{C}_{18} \\
\mathrm{H}_{30}\end{array}$ & $\begin{array}{c}\text { Aromat } \\
\text { ic }\end{array}$ & $\begin{array}{c}2211 . \\
8, \\
1.432\end{array}$ \\
\hline 6 & $\begin{array}{c}4536 \\
-86- \\
1\end{array}$ & Benzene, (1-propyloctyl)- & $\begin{array}{l}\mathrm{C}_{17} \\
\mathrm{H}_{28}\end{array}$ & $\begin{array}{l}\text { Aromat } \\
\text { ic }\end{array}$ & $\begin{array}{c}2079 . \\
81 \\
1.412\end{array}$ \\
\hline 6 & $\begin{array}{l}622- \\
96-8\end{array}$ & Benzene, 1-ethyl-4-methyl- & $\mathrm{C}_{9} \mathrm{H}_{12}$ & $\begin{array}{c}\text { Aromat } \\
\text { ic }\end{array}$ & $\begin{array}{c}1059 . \\
91 \\
1.188\end{array}$ \\
\hline 5 & $\begin{array}{c}2719 \\
-63- \\
3\end{array}$ & Benzene, (1-butyloctyl)- & $\begin{array}{l}\mathrm{C}_{18} \\
\mathrm{H}_{30}\end{array}$ & $\begin{array}{c}\text { Aromat } \\
\text { ic }\end{array}$ & $\begin{array}{c}2219 . \\
8 \\
1.445\end{array}$ \\
\hline 3 & $\begin{array}{c}9634 \\
55\end{array}$ & Benzene, (1-butylhexyl)- & $\begin{array}{l}\mathrm{C}_{16} \\
\mathrm{H}_{26}\end{array}$ & $\begin{array}{l}\text { Aromat } \\
\text { ic }\end{array}$ & $\begin{array}{c}1899 . \\
83 \\
1.379\end{array}$ \\
\hline 3 & $\begin{array}{c}4536 \\
-87- \\
2\end{array}$ & Benzene, (1-ethylnonyl)- & $\begin{array}{l}\mathrm{C}_{17} \\
\mathrm{H}_{28}\end{array}$ & $\begin{array}{l}\text { Aromat } \\
\text { ic }\end{array}$ & $\begin{array}{c}2119 . \\
81 \\
1.432\end{array}$ \\
\hline 3 & $\begin{array}{c}9634 \\
86 \\
\end{array}$ & Benzene, (1-propylheptyl)- & $\begin{array}{l}\mathrm{C}_{16} \\
\mathrm{H}_{26} \\
\end{array}$ & $\begin{array}{c}\text { Aromat } \\
\text { ic }\end{array}$ & $\begin{array}{c}1919 . \\
83\end{array}$ \\
\hline
\end{tabular}




\begin{tabular}{|c|c|c|c|c|c|}
\hline & & & & & 1.373 \\
\hline 3 & $\begin{array}{l}103- \\
65-1\end{array}$ & Benzene, propyl- & $\mathrm{C}_{9} \mathrm{H}_{12}$ & $\begin{array}{l}\text { Aromat } \\
\text { ic }\end{array}$ & $\begin{array}{c}1051 . \\
91, \\
1.188\end{array}$ \\
\hline 2 & $\begin{array}{c}71- \\
43-2\end{array}$ & Benzene & $\mathrm{C}_{6} \mathrm{H}_{6}$ & $\begin{array}{l}\text { Aromat } \\
\text { ic }\end{array}$ & $\begin{array}{c}623.9 \\
56, \\
1.280\end{array}$ \\
\hline 2 & $\begin{array}{c}4621 \\
-36- \\
7\end{array}$ & Benzene, (1-ethyloctyl)- & $\begin{array}{l}\mathrm{C}_{16} \\
\mathrm{H}_{26}\end{array}$ & $\begin{array}{l}\text { Aromat } \\
\text { ic }\end{array}$ & $\begin{array}{c}1951 . \\
82 \\
1.406\end{array}$ \\
\hline 2 & $\begin{array}{c}98- \\
82-8\end{array}$ & Benzene, (1-methylethyl)- & $\mathrm{C}_{9} \mathrm{H}_{12}$ & $\begin{array}{l}\text { Aromat } \\
\text { ic }\end{array}$ & $\begin{array}{c}1015 . \\
92, \\
1.175\end{array}$ \\
\hline 2 & $\begin{array}{c}5677 \\
76\end{array}$ & Benzene, 1-ethenyl-4-ethyl- & $\begin{array}{l}\mathrm{C}_{10} \\
\mathrm{H}_{12}\end{array}$ & $\begin{array}{l}\text { Aromat } \\
\text { ic }\end{array}$ & $\begin{array}{c}1219 . \\
9 \\
1.432\end{array}$ \\
\hline 2 & $\begin{array}{l}526- \\
73-8\end{array}$ & Benzene, 1,2,3-trimethyl- & $\mathrm{C}_{9} \mathrm{H}_{12}$ & $\begin{array}{l}\text { Aromat } \\
\text { ic }\end{array}$ & $\begin{array}{c}1075 . \\
91, \\
1.181\end{array}$ \\
\hline 2 & $\begin{array}{l}100- \\
42-5\end{array}$ & Styrene & $\mathrm{C}_{8} \mathrm{H}_{8}$ & $\begin{array}{l}\text { Aromat } \\
\text { ic }\end{array}$ & $\begin{array}{c}979.9 \\
2, \\
1.333\end{array}$ \\
\hline 1 & $\begin{array}{c}2400 \\
-00- \\
2\end{array}$ & Benzene, (1-ethyldecyl)- & $\begin{array}{l}\mathrm{C}_{18} \\
\mathrm{H}_{30}\end{array}$ & $\begin{array}{l}\text { Aromat } \\
\text { ic }\end{array}$ & $\begin{array}{c}2287 . \\
79 \\
1.538\end{array}$ \\
\hline 1 & $\begin{array}{c}4536 \\
-88- \\
3\end{array}$ & Benzene, (1-methyldecyl)- & $\begin{array}{l}\mathrm{C}_{17} \\
\mathrm{H}_{28}\end{array}$ & $\begin{array}{l}\text { Aromat } \\
\text { ic }\end{array}$ & $\begin{array}{c}2187 . \\
8 \\
1.478\end{array}$ \\
\hline 1 & $\begin{array}{c}2719 \\
-64- \\
4\end{array}$ & Benzene, (1-propylnonyl)- & $\begin{array}{l}\mathrm{C}_{18} \\
\mathrm{H}_{30}\end{array}$ & $\begin{array}{l}\text { Aromat } \\
\text { ic }\end{array}$ & $\begin{array}{c}2243 . \\
79 \\
1.478\end{array}$ \\
\hline 1 & $\begin{array}{c}6114 \\
2- \\
17-4\end{array}$ & Benzene, (1,2,2-trimethyl-3-butenyl)- & $\begin{array}{l}\mathrm{C}_{13} \\
\mathrm{H}_{18}\end{array}$ & $\begin{array}{l}\text { Aromat } \\
\text { ic }\end{array}$ & $\begin{array}{c}2015 . \\
82, \\
1.452\end{array}$ \\
\hline 1 & $\begin{array}{c}7525 \\
-62- \\
4\end{array}$ & Benzene, 1-ethenyl-3-ethyl- & $\begin{array}{l}\mathrm{C}_{10} \\
\mathrm{H}_{12}\end{array}$ & $\begin{array}{l}\text { Aromat } \\
\text { ic }\end{array}$ & $\begin{array}{c}1219 . \\
9, \\
1.432\end{array}$ \\
\hline 1 & $\begin{array}{l}611- \\
14-3\end{array}$ & Benzene, 1-ethyl-2-methyl- & $\mathrm{C}_{9} \mathrm{H}_{12}$ & $\begin{array}{l}\text { Aromat } \\
\text { ic }\end{array}$ & $\begin{array}{c}1087 . \\
91 \\
1.195\end{array}$ \\
\hline 1 & $\begin{array}{l}620- \\
14-4\end{array}$ & Benzene, 1-ethyl-3-methyl- & $\mathrm{C}_{9} \mathrm{H}_{12}$ & $\begin{array}{l}\text { Aromat } \\
\text { ic }\end{array}$ & $\begin{array}{c}1087 . \\
91 \\
1.201\end{array}$ \\
\hline 1 & $\begin{array}{l}535- \\
77-3\end{array}$ & Benzene, 1-methyl-3-(1-methylethyl)- & $\begin{array}{l}\mathrm{C}_{10} \\
\mathrm{H}_{14}\end{array}$ & $\begin{array}{l}\text { Aromat } \\
\text { ic }\end{array}$ & $\begin{array}{c}1135 . \\
9 \\
1.214\end{array}$ \\
\hline 1 & $\begin{array}{c}95- \\
63-6\end{array}$ & Benzene, 1,2,4-trimethyl- & $\mathrm{C}_{9} \mathrm{H}_{12}$ & $\begin{array}{l}\text { Aromat } \\
\text { ic }\end{array}$ & $\begin{array}{c}1075 . \\
91, \\
1.201\end{array}$ \\
\hline 1 & $\begin{array}{c}1758 \\
-88- \\
9\end{array}$ & Benzene, 2-ethyl-1,4-dimethyl- & $\begin{array}{l}\mathrm{C}_{10} \\
\mathrm{H}_{14}\end{array}$ & $\begin{array}{l}\text { Aromat } \\
\text { ic }\end{array}$ & $\begin{array}{c}1179 . \\
9 \\
1.267\end{array}$ \\
\hline
\end{tabular}




\begin{tabular}{|c|c|c|c|c|c|}
\hline 20 & $\begin{array}{c}7438 \\
1- \\
40-1\end{array}$ & $\begin{array}{l}\text { Propanoic acid, 2-methyl-, 1-(1,1-dimethylethyl)- } \\
\text { 2-methyl-1,3-propanediyl ester }\end{array}$ & $\begin{array}{c}\mathrm{C}_{16} \\
\mathrm{H}_{30} \mathrm{O}_{4}\end{array}$ & Ester & $\begin{array}{c}2019 . \\
82 \\
1.472\end{array}$ \\
\hline 19 & $\begin{array}{l}7436 \\
7- \\
33-2\end{array}$ & $\begin{array}{l}\text { Propanoic acid, 2-methyl-, 2,2-dimethyl-1-(2- } \\
\text { hydroxy-1-methylethyl)propyl ester }\end{array}$ & $\begin{array}{c}\mathrm{C}_{12} \\
\mathrm{H}_{24} \mathrm{O}_{3}\end{array}$ & Ester & $\begin{array}{c}1635 . \\
85 \\
2.006\end{array}$ \\
\hline 19 & $\begin{array}{c}7436 \\
7- \\
34-3\end{array}$ & $\begin{array}{l}\text { Propanoic acid, 2-methyl-, 3-hydroxy-2,4,4- } \\
\text { trimethylpentyl ester }\end{array}$ & $\begin{array}{c}\mathrm{C}_{12} \\
\mathrm{H}_{24} \mathrm{O}_{3}\end{array}$ & Ester & $\begin{array}{c}1655 . \\
85, \\
1.855\end{array}$ \\
\hline 14 & $\begin{array}{l}105- \\
37-3\end{array}$ & Propanoic acid, ethyl ester & $\begin{array}{c}\mathrm{C}_{5} \mathrm{H}_{10} \\
\mathrm{O}_{2}\end{array}$ & Ester & $\begin{array}{l}687.9 \\
49 \\
1.208\end{array}$ \\
\hline 9 & $\begin{array}{l}624- \\
54-4\end{array}$ & Propanoic acid, pentyl ester & $\begin{array}{c}\mathrm{C}_{8} \mathrm{H}_{16} \\
\mathrm{O}_{2}\end{array}$ & Ester & $\begin{array}{c}1087 . \\
91 \\
1.142\end{array}$ \\
\hline 8 & $\begin{array}{c}6846 \\
-50- \\
0\end{array}$ & 2,2,4-Trimethyl-1,3-pentanediol diisobutyrate & $\begin{array}{c}\mathrm{C}_{16} \\
\mathrm{H}_{30} \mathrm{O}_{4}\end{array}$ & Ester & $\begin{array}{l}1635 . \\
85 \\
2.000\end{array}$ \\
\hline 8 & $\begin{array}{l}123- \\
66-0\end{array}$ & Hexanoic acid, ethyl ester & $\begin{array}{c}\mathrm{C}_{8} \mathrm{H}_{16} \\
\mathrm{O}_{2}\end{array}$ & Ester & $\begin{array}{c}1079 . \\
91 \\
1.135\end{array}$ \\
\hline 7 & $\begin{array}{c}0- \\
00-0\end{array}$ & Propanoic acid, 2-penten-1-yl ester (Z)- & $\begin{array}{c}\mathrm{C}_{8} \mathrm{H}_{14} \\
\mathrm{O}_{2}\end{array}$ & Ester & $\begin{array}{c}1087 . \\
91 \\
1.181\end{array}$ \\
\hline 3 & $\begin{array}{c}2408 \\
-20- \\
0\end{array}$ & Propanoic acid, 2-propenyl ester & $\begin{array}{c}\mathrm{C}_{6} \mathrm{H}_{10} \\
\mathrm{O}_{2}\end{array}$ & Ester & $\begin{array}{c}1103 . \\
91 \\
1.419\end{array}$ \\
\hline 2 & $\begin{array}{l}105- \\
90-8\end{array}$ & 2,6-Octadien-1-ol, 3,7-dimethyl-, propanoate, (E)- & $\begin{array}{c}\mathrm{C}_{13} \\
\mathrm{H}_{22} \mathrm{O}_{2}\end{array}$ & Ester & $\begin{array}{c}1103 . \\
91 \\
1.089\end{array}$ \\
\hline 2 & $\begin{array}{l}689- \\
12-3\end{array}$ & Isopropyl acrylate & $\begin{array}{c}\mathrm{C}_{6} \mathrm{H}_{10} \\
\mathrm{O}_{2}\end{array}$ & Ester & $\begin{array}{l}1427 . \\
88 \\
1.914\end{array}$ \\
\hline 1 & $\begin{array}{c}4814 \\
5- \\
04-6\end{array}$ & -Phenoxyethyl acrylate & $\begin{array}{c}\mathrm{C}_{11} \\
\mathrm{H}_{12} \mathrm{O}_{3}\end{array}$ & Ester & $\begin{array}{c}1795 . \\
84, \\
2.891\end{array}$ \\
\hline 1 & $\begin{array}{l}105- \\
68-0\end{array}$ & 1-Butanol, 3-methyl-, propanoate & $\begin{array}{c}\mathrm{C}_{8} \mathrm{H}_{16} \\
\mathrm{O}_{2}\end{array}$ & Ester & $\begin{array}{c}1087 . \\
91 \\
1.135\end{array}$ \\
\hline 1 & $\begin{array}{c}84- \\
69-5\end{array}$ & $\begin{array}{l}\text { 1,2-Benzenedicarboxylic acid, bis(2- } \\
\text { methylpropyl) ester }\end{array}$ & $\begin{array}{c}\mathrm{C}_{16} \\
\mathrm{H}_{22} \mathrm{O}_{4}\end{array}$ & Ester & $\begin{array}{l}2515 . \\
77 \\
3.287\end{array}$ \\
\hline 1 & $\begin{array}{c}4212 \\
5- \\
10-0\end{array}$ & 2-Penten-1-ol, acetate, $(\mathrm{Z})$ - & $\begin{array}{c}\mathrm{C}_{7} \mathrm{H}_{12} \\
\mathrm{O}_{2}\end{array}$ & Ester & $\begin{array}{c}875.9 \\
3 \\
1.175\end{array}$ \\
\hline 1 & $\begin{array}{l}818- \\
61-1\end{array}$ & 2-Propenoic acid, 2-hydroxyethyl ester & $\begin{array}{c}\mathrm{C}_{5} \mathrm{H}_{8} \\
\mathrm{O}_{3}\end{array}$ & Ester & $\begin{array}{l}1003 . \\
92 \\
2.911\end{array}$ \\
\hline 1 & $\begin{array}{c}1389 \\
4- \\
61-6\end{array}$ & 3-Hexenoic acid, methyl ester, (E)- & $\begin{array}{c}\mathrm{C}_{7} \mathrm{H}_{12} \\
\mathrm{O}_{2}\end{array}$ & Ester & $\begin{array}{l}999.9 \\
18 \\
1.228\end{array}$ \\
\hline 1 & 6966 & 3-Octen-1-ol, acetate, (Z)- & $\mathrm{C}_{10}$ & Ester & 1475. \\
\hline
\end{tabular}




\begin{tabular}{|c|c|c|c|c|c|}
\hline & $\begin{array}{c}8- \\
83-3\end{array}$ & & $\mathrm{H}_{18} \mathrm{O}_{2}$ & & $\begin{array}{c}87 \\
1.525\end{array}$ \\
\hline 1 & $\begin{array}{c}0- \\
00-0\end{array}$ & 4-Ethylbenzoic acid, cyclopentyl ester & $\begin{array}{c}\mathrm{C}_{14} \\
\mathrm{H}_{18} \mathrm{O}_{2}\end{array}$ & Ester & $\begin{array}{c}1015 . \\
92 \\
2.449\end{array}$ \\
\hline 1 & $\begin{array}{c}2113 \\
91\end{array}$ & 4-Hydroxybutyl acrylate & $\begin{array}{c}\mathrm{C}_{7} \mathrm{H}_{12} \\
\mathrm{O}_{3}\end{array}$ & Ester & $\begin{array}{c}1791 . \\
84 \\
0.119\end{array}$ \\
\hline 1 & $\begin{array}{c}2396 \\
-80- \\
7\end{array}$ & 5-Hexenoic acid, methyl ester & $\begin{array}{c}\mathrm{C}_{7} \mathrm{H}_{12} \\
\mathrm{O}_{2}\end{array}$ & Ester & $\begin{array}{c}999.9 \\
18 \\
1.234\end{array}$ \\
\hline 1 & $\begin{array}{l}108- \\
05-4\end{array}$ & Acetic acid ethenyl ester & $\begin{array}{c}\mathrm{C}_{4} \mathrm{H}_{6} \\
\mathrm{O}_{2}\end{array}$ & Ester & $\begin{array}{c}479.9 \\
7, \\
1.709\end{array}$ \\
\hline 1 & $\begin{array}{c}1694 \\
-31- \\
1\end{array}$ & Butanoic acid, 3-oxo-, 1,1-dimethylethyl ester & $\begin{array}{c}\mathrm{C}_{8} \mathrm{H}_{14} \\
\mathrm{O}_{3}\end{array}$ & Ester & $\begin{array}{c}1259 . \\
89 \\
1.861\end{array}$ \\
\hline 1 & $\begin{array}{c}84- \\
66-2\end{array}$ & Diethyl Phthalate & $\begin{array}{c}\mathrm{C}_{12} \\
\mathrm{H}_{14} \mathrm{O}_{4}\end{array}$ & Ester & $\begin{array}{c}2023 . \\
82, \\
3.010\end{array}$ \\
\hline 1 & $\begin{array}{l}100- \\
41-4\end{array}$ & Ethylbenzene & $\mathrm{C}_{8} \mathrm{H}_{10}$ & Ester & $\begin{array}{c}935.9 \\
24, \\
1.208\end{array}$ \\
\hline 1 & $\begin{array}{l}692- \\
45-5\end{array}$ & Formic acid, ethenyl ester & $\begin{array}{c}\mathrm{C}_{3} \mathrm{H}_{4} \\
\mathrm{O}_{2}\end{array}$ & Ester & $\begin{array}{c}223.9 \\
96, \\
1.261\end{array}$ \\
\hline 1 & $\begin{array}{l}816- \\
19-3\end{array}$ & Hexanoic acid, 2-ethyl-, methyl ester & $\begin{array}{c}\mathrm{C}_{9} \mathrm{H}_{18} \\
\mathrm{O}_{2}\end{array}$ & Ester & $\begin{array}{c}1131 . \\
9 \\
1.148\end{array}$ \\
\hline 1 & $\begin{array}{c}3050 \\
-69- \\
9\end{array}$ & n-Caproic acid vinyl ester & $\begin{array}{c}\mathrm{C}_{8} \mathrm{H}_{14} \\
\mathrm{O}_{2}\end{array}$ & Ester & $\begin{array}{c}1215 . \\
9 \\
1.914\end{array}$ \\
\hline 1 & $\begin{array}{c}0- \\
00-0\end{array}$ & Oxalic acid, isobutyl heptyl ester & $\begin{array}{c}\mathrm{C}_{13} \\
\mathrm{H}_{24} \mathrm{O}_{4}\end{array}$ & Ester & $\begin{array}{c}2007 . \\
82 \\
1.267\end{array}$ \\
\hline 1 & $\begin{array}{c}0- \\
00-0\end{array}$ & $\begin{array}{l}\text { Pentanoic acid, 2,2,4-trimethyl-3- } \\
\text { carboxyisopropyl, isobutyl ester }\end{array}$ & $\begin{array}{c}\mathrm{C}_{16} \\
\mathrm{H}_{30} \mathrm{O}_{4}\end{array}$ & Ester & $\begin{array}{c}2023 . \\
82, \\
1.452\end{array}$ \\
\hline 1 & $\begin{array}{c}0- \\
00-0\end{array}$ & Phthalic acid, butyl hexyl ester & $\begin{array}{c}\mathrm{C}_{18} \\
\mathrm{H}_{26} \mathrm{O}_{4}\end{array}$ & Ester & $\begin{array}{c}2751 . \\
74, \\
0.350\end{array}$ \\
\hline 1 & $\begin{array}{l}108- \\
59-8\end{array}$ & Propanedioic acid, dimethyl ester & $\begin{array}{c}\mathrm{C}_{5} \mathrm{H}_{8} \\
\mathrm{O}_{4}\end{array}$ & Ester & $\begin{array}{c}1079 . \\
91 \\
1.267\end{array}$ \\
\hline 1 & $\begin{array}{c}1638 \\
7- \\
18-1\end{array}$ & Propanoic acid, 2,2-dimethyl-, 2-ethylhexyl ester & $\begin{array}{c}\mathrm{C}_{13} \\
\mathrm{H}_{26} \mathrm{O}_{2}\end{array}$ & Ester & $\begin{array}{c}2007 . \\
82 \\
1.254\end{array}$ \\
\hline 8 & $\begin{array}{l}629- \\
82-3\end{array}$ & Octane, 1,1'-oxybis- & $\begin{array}{c}\mathrm{C}_{16} \\
\mathrm{H}_{34} \mathrm{O}\end{array}$ & Ether & $\begin{array}{c}2083 . \\
81 \\
1.247\end{array}$ \\
\hline 7 & $\begin{array}{l}112- \\
36-7\end{array}$ & Ethane, 1,1'-oxybis[2-ethoxy-] & $\begin{array}{c}\mathrm{C}_{8} \mathrm{H}_{18} \\
\mathrm{O}_{3} \\
\end{array}$ & Ether & $\begin{array}{c}855.9 \\
32\end{array}$ \\
\hline
\end{tabular}




\begin{tabular}{|c|c|c|c|c|c|}
\hline & & & & & 1.485 \\
\hline 3 & $\begin{array}{c}1504 \\
5- \\
60-0\end{array}$ & Pentane, 1,3-epoxy-4-methyl- & $\begin{array}{l}\mathrm{C}_{6} \mathrm{H}_{12} \\
\mathrm{O}\end{array}$ & Ether & $\begin{array}{c}1075 . \\
91, \\
1.175\end{array}$ \\
\hline 2 & $\begin{array}{l}627- \\
08-7\end{array}$ & Propane, 1-(1-methylethoxy)- & $\begin{array}{l}\mathrm{C}_{6} \mathrm{H}_{14} \\
\mathrm{O}\end{array}$ & Ether & $\begin{array}{c}1279 . \\
89 \\
1.505\end{array}$ \\
\hline 1 & $\begin{array}{c}0- \\
00-0\end{array}$ & 1-Hexene, 3-methyl-6-phenyl-4-(1-phenylethoxy)- & $\begin{array}{c}\mathrm{C}_{21} \\
\mathrm{H}_{26} \mathrm{O}\end{array}$ & Ether & $\begin{array}{c}2183 . \\
8, \\
1.485\end{array}$ \\
\hline 1 & $\begin{array}{l}116- \\
11-0\end{array}$ & 1-Propene, 2-methoxy- & $\begin{array}{l}\mathrm{C}_{4} \mathrm{H}_{8} \\
\mathrm{O}\end{array}$ & Ether & $\begin{array}{c}699.9 \\
48, \\
1.102\end{array}$ \\
\hline 1 & $\begin{array}{c}2020 \\
2- \\
62-4\end{array}$ & 1,7-Octadiene, 3-methoxy- & $\begin{array}{l}\mathrm{C}_{9} \mathrm{H}_{16} \\
\mathrm{O}\end{array}$ & Ether & $\begin{array}{c}899.9 \\
28, \\
1.043\end{array}$ \\
\hline 1 & $\begin{array}{c}1428 \\
9- \\
96-4\end{array}$ & Allyl methallyl ether & $\begin{array}{c}\mathrm{C}_{7} \mathrm{H}_{12} \\
\mathrm{O}\end{array}$ & Ether & $\begin{array}{c}815.9 \\
36, \\
1.003\end{array}$ \\
\hline 1 & $\begin{array}{c}1860 \\
-27- \\
1\end{array}$ & Butane, 1-(1-methylethoxy)- & $\begin{array}{l}\mathrm{C}_{7} \mathrm{H}_{16} \\
\mathrm{O}\end{array}$ & Ether & $\begin{array}{c}1279 . \\
89 \\
1.492\end{array}$ \\
\hline 1 & $\begin{array}{c}3235 \\
7- \\
83-8\end{array}$ & Ether, hexyl pentyl & $\begin{array}{c}\mathrm{C}_{11} \\
\mathrm{H}_{24} \mathrm{O}\end{array}$ & Ether & $\begin{array}{c}2071 . \\
81 \\
1.129\end{array}$ \\
\hline 1 & $\begin{array}{c}5400 \\
4- \\
26-1\end{array}$ & Pentane, 1-(2-butenyloxy)-, (E)- & $\begin{array}{l}\mathrm{C}_{9} \mathrm{H}_{18} \\
\mathrm{O}\end{array}$ & Ether & $\begin{array}{c}1087 . \\
91, \\
1.142\end{array}$ \\
\hline 17 & $\begin{array}{c}3777 \\
-69- \\
3\end{array}$ & Furan, 2-pentyl- & $\begin{array}{l}\mathrm{C}_{9} \mathrm{H}_{14} \\
\mathrm{O}\end{array}$ & Furan & $\begin{array}{c}1079 . \\
91, \\
1.142\end{array}$ \\
\hline 12 & $\begin{array}{c}3208 \\
-16- \\
0\end{array}$ & Furan, 2-ethyl- & $\begin{array}{c}\mathrm{C}_{6} \mathrm{H}_{8} \\
\mathrm{O}\end{array}$ & Furan & $\begin{array}{c}679.9 \\
5, \\
1.221\end{array}$ \\
\hline 9 & $\begin{array}{l}534- \\
22-5\end{array}$ & Furan, 2-methyl- & $\begin{array}{l}\mathrm{C}_{5} \mathrm{H}_{6} \\
\mathrm{O}\end{array}$ & Furan & $\begin{array}{c}507.9 \\
67, \\
1.294\end{array}$ \\
\hline 9 & $\begin{array}{c}4229 \\
-91- \\
8\end{array}$ & Furan, 2-propyl- & $\begin{array}{l}\mathrm{C}_{7} \mathrm{H}_{10} \\
\mathrm{O}\end{array}$ & Furan & $\begin{array}{c}819.9 \\
36, \\
1.168\end{array}$ \\
\hline 8 & $\begin{array}{l}695- \\
06-7\end{array}$ & 2(3H)-Furanone, 5-ethyldihydro- & $\begin{array}{c}\mathrm{C}_{6} \mathrm{H}_{10} \\
\mathrm{O}_{2}\end{array}$ & Furan & $\begin{array}{c}1175 . \\
9 \\
2.383\end{array}$ \\
\hline 5 & $\begin{array}{c}4466 \\
-24- \\
4\end{array}$ & 2-n-Butyl furan & $\begin{array}{c}\mathrm{C}_{8} \mathrm{H}_{12} \\
\mathrm{O}\end{array}$ & Furan & $\begin{array}{c}959.9 \\
22, \\
1.155\end{array}$ \\
\hline 4 & $\begin{array}{c}5371 \\
-52- \\
8\end{array}$ & 2-Furanol, tetrahydro- & $\begin{array}{c}\mathrm{C}_{4} \mathrm{H}_{8} \\
\mathrm{O}_{2}\end{array}$ & Furan & $\begin{array}{c}991.9 \\
19, \\
2.158\end{array}$ \\
\hline 4 & $\begin{array}{c}2407 \\
-43- \\
4\end{array}$ & 2(5H)-Furanone, 5-ethyl- & $\begin{array}{c}\mathrm{C}_{6} \mathrm{H}_{8} \\
\mathrm{O}_{2}\end{array}$ & Furan & $\begin{array}{c}1151 . \\
9 \\
2.640\end{array}$ \\
\hline
\end{tabular}




\begin{tabular}{|c|c|c|c|c|c|}
\hline 3 & $\begin{array}{l}109- \\
99-9\end{array}$ & Furan, tetrahydro- & $\begin{array}{c}\mathrm{C}_{4} \mathrm{H}_{8} \\
\mathrm{O}\end{array}$ & Furan & $\begin{array}{l}599.9 \\
58 \\
1.168\end{array}$ \\
\hline 2 & $\begin{array}{c}1193 \\
-79- \\
9\end{array}$ & 2-Acetyl-5-methylfuran & $\begin{array}{c}\mathrm{C}_{7} \mathrm{H}_{8} \\
\mathrm{O}_{2}\end{array}$ & Furan & $\begin{array}{c}947.9 \\
23, \\
1.122\end{array}$ \\
\hline 2 & $\begin{array}{c}7042 \\
4- \\
14-5\end{array}$ & trans-2-(2-Pentenyl)furan & $\begin{array}{c}\mathrm{C}_{9} \mathrm{H}_{12} \\
\mathrm{O}\end{array}$ & Furan & $\begin{array}{c}1091 . \\
91 \\
1.208\end{array}$ \\
\hline 1 & $\begin{array}{c}5989 \\
-33- \\
3\end{array}$ & $\begin{array}{l}\text { 2-Furanmethanol, 5-ethenyltetrahydro- } \$, \ddagger, 5 \text { - } \\
\text { trimethyl-, cis- }\end{array}$ & $\begin{array}{c}\mathrm{C}_{10} \\
\mathrm{H}_{18} \mathrm{O}_{2}\end{array}$ & Furan & $\begin{array}{c}1191 . \\
9 \\
1.432\end{array}$ \\
\hline 1 & $\begin{array}{c}1191 \\
-99- \\
7\end{array}$ & 2,3-Dihydrofuran & $\begin{array}{c}\mathrm{C}_{4} \mathrm{H}_{6} \\
\mathrm{O}\end{array}$ & Furan & $\begin{array}{l}463.9 \\
72 \\
1.307\end{array}$ \\
\hline 1 & $\begin{array}{l}108- \\
29-2\end{array}$ & 2(3H)-Furanone, dihydro-5-methyl- & $\begin{array}{c}\mathrm{C}_{5} \mathrm{H}_{8} \\
\mathrm{O}_{2}\end{array}$ & Furan & $\begin{array}{c}1051 . \\
91 \\
2.138\end{array}$ \\
\hline 1 & $\begin{array}{l}539- \\
52-6\end{array}$ & Furan, 3-(4-methyl-3-pentenyl)- & $\begin{array}{c}\mathrm{C}_{10} \\
\mathrm{H}_{14} \mathrm{O}\end{array}$ & Furan & $\begin{array}{c}1219 . \\
9 \\
1.346\end{array}$ \\
\hline 1 & $\begin{array}{c}0- \\
00-0\end{array}$ & $\begin{array}{l}\text { Spiro[2,4,5,6,7,7a-hexahydro-2-oxo- } 4,4,7 \mathrm{a} \text { - } \\
\quad \text { trimethylbenzofuran]-7,2'-(oxirane) }\end{array}$ & $\begin{array}{c}\mathrm{C}_{12} \\
\mathrm{H}_{16} \mathrm{O}_{3}\end{array}$ & Furan & $\begin{array}{c}1803 . \\
84 \\
2.204\end{array}$ \\
\hline 1 & $\begin{array}{l}928- \\
50-7\end{array}$ & 1-Pentene, 5-chloro- & $\begin{array}{l}\mathrm{C}_{5} \mathrm{H}_{9} \\
\mathrm{Cl}\end{array}$ & $\begin{array}{l}\text { Haloge } \\
\mathrm{n}\end{array}$ & $\begin{array}{c}767.9 \\
41, \\
1.201\end{array}$ \\
\hline 1 & $\begin{array}{c}1763 \\
-21- \\
9\end{array}$ & 1,5-Hexadiene, 3,3,4,4-tetrafluoro- & $\begin{array}{c}\mathrm{C}_{6} \mathrm{H}_{6} \\
\mathrm{~F}_{4}\end{array}$ & $\begin{array}{c}\text { Haloge } \\
n\end{array}$ & $\begin{array}{c}779.9 \\
4, \\
1.333\end{array}$ \\
\hline 1 & $\begin{array}{l}998- \\
93-6\end{array}$ & 4-Bromoheptane & $\begin{array}{c}\mathrm{C}_{7} \mathrm{H}_{15} \\
\mathrm{Br}\end{array}$ & $\begin{array}{c}\text { Haloge } \\
n\end{array}$ & $\begin{array}{c}1071 . \\
91 \\
1.221\end{array}$ \\
\hline 1 & $\begin{array}{l}407- \\
25-0\end{array}$ & Acetic acid, trifluoro-, anhydride & $\begin{array}{c}\mathrm{C}_{4} \mathrm{~F}_{6} \\
\mathrm{O}_{3}\end{array}$ & $\begin{array}{c}\text { Haloge } \\
n\end{array}$ & $\begin{array}{c}1071 . \\
91, \\
1.267\end{array}$ \\
\hline 1 & $\begin{array}{l}352- \\
70-5\end{array}$ & Benzene, 1-fluoro-3-methyl- & $\begin{array}{c}\mathrm{C}_{7} \mathrm{H}_{7} \\
\mathrm{~F}\end{array}$ & $\begin{array}{c}\text { Haloge } \\
n\end{array}$ & $\begin{array}{c}1023 . \\
92, \\
1.023\end{array}$ \\
\hline 1 & $\begin{array}{l}541- \\
73-1\end{array}$ & Benzene, 1,3-dichloro- & $\begin{array}{c}\mathrm{C}_{6} \mathrm{H}_{4} \\
\mathrm{Cl}_{2}\end{array}$ & $\begin{array}{c}\text { Haloge } \\
n\end{array}$ & $\begin{array}{c}1131 . \\
9 \\
1.505\end{array}$ \\
\hline 1 & $\begin{array}{l}100- \\
44-7\end{array}$ & Benzyl chloride & $\begin{array}{c}\mathrm{C}_{7} \mathrm{H}_{7} \\
\mathrm{Cl}\end{array}$ & $\begin{array}{c}\text { Haloge } \\
n\end{array}$ & $\begin{array}{c}1127 . \\
91, \\
1.643\end{array}$ \\
\hline 1 & $\begin{array}{l}107- \\
82-4\end{array}$ & Butane, 1-bromo-3-methyl- & $\begin{array}{c}\mathrm{C}_{5} \mathrm{H}_{11} \\
\mathrm{Br}\end{array}$ & $\begin{array}{c}\text { Haloge } \\
n\end{array}$ & $\begin{array}{c}1067 . \\
91 \\
1.228\end{array}$ \\
\hline 1 & $\begin{array}{l}616- \\
13-7\end{array}$ & Butane, 1-chloro-2-methyl- & $\begin{array}{c}\mathrm{C}_{5} \mathrm{H}_{11} \\
\mathrm{Cl}\end{array}$ & $\begin{array}{c}\text { Haloge } \\
n\end{array}$ & $\begin{array}{c}1047 . \\
91, \\
1.432\end{array}$ \\
\hline 1 & 971- & Chlorotris(p-tolyl)methane & $\mathrm{C}_{22}$ & Haloge & 1387. \\
\hline
\end{tabular}




\begin{tabular}{|c|c|c|c|c|c|}
\hline & $93-7$ & & $\mathrm{H}_{21} \mathrm{Cl}$ & $\mathrm{n}$ & 88 \\
\hline 1 & $\begin{array}{l}137- \\
43-9\end{array}$ & Cyclopentane, bromo- & $\begin{array}{c}\mathrm{C}_{5} \mathrm{H}_{9} \\
\mathrm{Br}\end{array}$ & $\begin{array}{c}\text { Haloge } \\
n\end{array}$ & $\begin{array}{c}1071 . \\
91 \\
1.023\end{array}$ \\
\hline 1 & $\begin{array}{l}629- \\
06-1\end{array}$ & Heptane, 1-chloro- & $\begin{array}{c}\mathrm{C}_{7} \mathrm{H}_{15} \\
\mathrm{Cl}\end{array}$ & $\begin{array}{c}\text { Haloge } \\
n\end{array}$ & $\begin{array}{c}1167 . \\
9 \\
1.155\end{array}$ \\
\hline 1 & $\begin{array}{l}648- \\
36-2\end{array}$ & Hexane, 3,3,4,4-tetrafluoro- & $\begin{array}{c}\mathrm{C}_{6} \mathrm{H}_{10} \\
\mathrm{~F}_{4}\end{array}$ & $\begin{array}{c}\text { Haloge } \\
n\end{array}$ & $\begin{array}{c}387.9 \\
79, \\
2.581\end{array}$ \\
\hline 1 & $\begin{array}{l}999- \\
06-4\end{array}$ & Octane, 4-bromo- & $\begin{array}{c}\mathrm{C}_{8} \mathrm{H}_{17} \\
\mathrm{Br}\end{array}$ & $\begin{array}{c}\text { Haloge } \\
n\end{array}$ & $\begin{array}{c}1075 . \\
91, \\
1.129\end{array}$ \\
\hline 1 & $\begin{array}{c}8601 \\
2- \\
32-0\end{array}$ & Phosphonous dibromide, cyclohexyl- & $\begin{array}{l}\mathrm{C}_{6} \mathrm{H}_{11} \\
\mathrm{Br}_{2} \mathrm{P}\end{array}$ & $\begin{array}{c}\text { Haloge } \\
n\end{array}$ & $\begin{array}{c}1671 . \\
85, \\
1.294\end{array}$ \\
\hline 1 & $\begin{array}{l}703- \\
13-9\end{array}$ & Trifluoroacetic acid, cyclopentyl ester & $\begin{array}{l}\mathrm{C}_{7} \mathrm{H}_{9} \\
\mathrm{~F}_{3} \mathrm{O}_{2}\end{array}$ & $\begin{array}{c}\text { Haloge } \\
n\end{array}$ & $\begin{array}{c}1103 . \\
91, \\
1.089\end{array}$ \\
\hline 18 & $\begin{array}{c}96- \\
22-0\end{array}$ & 3-Pentanone & $\begin{array}{c}\mathrm{C}_{5} \mathrm{H}_{10} \\
\mathrm{O}\end{array}$ & Ketone & $\begin{array}{c}1035 . \\
91, \\
1.868\end{array}$ \\
\hline 17 & $\begin{array}{l}106- \\
68-3\end{array}$ & 3-Octanone & $\begin{array}{c}\mathrm{C}_{8} \mathrm{H}_{16} \\
\mathrm{O}\end{array}$ & Ketone & $\begin{array}{c}1071 . \\
91, \\
1.181\end{array}$ \\
\hline 14 & $\begin{array}{c}1629 \\
-58- \\
9\end{array}$ & 1-Penten-3-one & $\begin{array}{c}\mathrm{C}_{5} \mathrm{H}_{8} \\
\mathrm{O}\end{array}$ & Ketone & $\begin{array}{c}1067 . \\
91, \\
1.016\end{array}$ \\
\hline 12 & $\begin{array}{c}1190 \\
-34- \\
7\end{array}$ & 5-Hepten-3-one, 5-methyl- & $\begin{array}{c}\mathrm{C}_{8} \mathrm{H}_{14} \\
\mathrm{O}\end{array}$ & Ketone & $\begin{array}{c}1075 . \\
91, \\
1.254\end{array}$ \\
\hline 10 & $\begin{array}{l}107- \\
87-9\end{array}$ & 2-Pentanone & $\begin{array}{c}\mathrm{C}_{5} \mathrm{H}_{10} \\
\mathrm{O}\end{array}$ & Ketone & $\begin{array}{c}655.9 \\
52, \\
1.307\end{array}$ \\
\hline 8 & $\begin{array}{c}98- \\
86-2\end{array}$ & Acetophenone & $\begin{array}{c}\mathrm{C}_{8} \mathrm{H}_{8} \\
\mathrm{O}\end{array}$ & Ketone & $\begin{array}{c}1203 . \\
9, \\
2.105\end{array}$ \\
\hline 7 & $\begin{array}{l}141- \\
79-7\end{array}$ & 3-Penten-2-one, 4-methyl- & $\begin{array}{c}\mathrm{C}_{6} \mathrm{H}_{10} \\
\mathrm{O}\end{array}$ & Ketone & $\begin{array}{c}839.9 \\
34, \\
1.327\end{array}$ \\
\hline 4 & $\begin{array}{c}78- \\
93-3\end{array}$ & 2-Butanone & $\begin{array}{c}\mathrm{C}_{4} \mathrm{H}_{8} \\
\mathrm{O}\end{array}$ & Ketone & $\begin{array}{c}495.9 \\
68, \\
1.412\end{array}$ \\
\hline 4 & $\begin{array}{c}2611 \\
8- \\
97-8\end{array}$ & 6-Hepten-3-one, 4-methyl- & $\begin{array}{c}\mathrm{C}_{8} \mathrm{H}_{14} \\
\mathrm{O}\end{array}$ & Ketone & $\begin{array}{c}1055 . \\
91, \\
1.254\end{array}$ \\
\hline 3 & $\begin{array}{l}513- \\
86-0\end{array}$ & 2-Butanone, 3-hydroxy- & $\begin{array}{c}\mathrm{C}_{4} \mathrm{H}_{8} \\
\mathrm{O}_{2}\end{array}$ & Ketone & $\begin{array}{c}691.9 \\
49, \\
2.482\end{array}$ \\
\hline 3 & $\begin{array}{c}78- \\
59-1 \\
\end{array}$ & 2-Cyclohexen-1-one, 3,5,5-trimethyl- & $\begin{array}{c}\mathrm{C}_{9} \mathrm{H}_{14} \\
\mathrm{O} \\
\end{array}$ & Ketone & $\begin{array}{c}1183 . \\
9\end{array}$ \\
\hline
\end{tabular}




\begin{tabular}{|c|c|c|c|c|c|}
\hline & & & & & 1.432 \\
\hline 3 & $\begin{array}{l}110- \\
43-0\end{array}$ & 2-Heptanone & $\begin{array}{c}\mathrm{C}_{7} \mathrm{H}_{14} \\
\mathrm{O}\end{array}$ & Ketone & $\begin{array}{c}959.9 \\
22, \\
1.221\end{array}$ \\
\hline 3 & $\begin{array}{c}1954 \\
9- \\
80-5\end{array}$ & 2-Heptanone, 4,6-dimethyl- & $\begin{array}{l}\mathrm{C}_{9} \mathrm{H}_{18} \\
\mathrm{O}\end{array}$ & Ketone & $\begin{array}{c}1687 . \\
85, \\
1.360\end{array}$ \\
\hline 3 & $\begin{array}{l}591- \\
78-6\end{array}$ & 2-Hexanone & $\begin{array}{c}\mathrm{C}_{6} \mathrm{H}_{12} \\
\mathrm{O}\end{array}$ & Ketone & $\begin{array}{c}819.9 \\
36, \\
1.247\end{array}$ \\
\hline 3 & $\begin{array}{c}4984 \\
-85- \\
4\end{array}$ & 4-Hydroxy-3-hexanone & $\begin{array}{c}\mathrm{C}_{6} \mathrm{H}_{12} \\
\mathrm{O}_{2}\end{array}$ & Ketone & $\begin{array}{c}1199 . \\
9, \\
1.775\end{array}$ \\
\hline 2 & $\begin{array}{c}4312 \\
-99- \\
6\end{array}$ & 1-Octen-3-one & $\begin{array}{l}\mathrm{C}_{8} \mathrm{H}_{14} \\
\mathrm{O}\end{array}$ & Ketone & $\begin{array}{c}1067 . \\
91, \\
1.221\end{array}$ \\
\hline 2 & $\begin{array}{l}563- \\
80-4\end{array}$ & 2-Butanone, 3-methyl- & $\begin{array}{l}\mathrm{C}_{5} \mathrm{H}_{10} \\
\quad \mathrm{O}\end{array}$ & Ketone & $\begin{array}{c}611.9 \\
57, \\
1.254\end{array}$ \\
\hline 2 & $\begin{array}{c}1187 \\
-87- \\
7\end{array}$ & 2-Hexanone, 5-methyl-3-methylene- & $\begin{array}{l}\mathrm{C}_{8} \mathrm{H}_{14} \\
\mathrm{O}\end{array}$ & Ketone & $\begin{array}{c}1039 . \\
91, \\
1.208\end{array}$ \\
\hline 2 & $\begin{array}{l}111- \\
13-7\end{array}$ & 2-Octanone & $\begin{array}{l}\mathrm{C}_{8} \mathrm{H}_{16} \\
\mathrm{O}\end{array}$ & Ketone & $\begin{array}{c}1499 . \\
87, \\
1.445\end{array}$ \\
\hline 2 & $\begin{array}{c}3674 \\
5- \\
27-4\end{array}$ & 2,3-Dimethoxy-2',4'-dihydroxychalcone & $\begin{array}{c}\mathrm{C}_{17} \\
\mathrm{H}_{16} \mathrm{O}_{5}\end{array}$ & Ketone & $\begin{array}{c}2363 . \\
78, \\
1.736\end{array}$ \\
\hline 2 & $\begin{array}{l}624- \\
42-0\end{array}$ & 3-Heptanone, 6-methyl- & $\begin{array}{l}\mathrm{C}_{8} \mathrm{H}_{16} \\
\mathrm{O}\end{array}$ & Ketone & $\begin{array}{c}1083 . \\
91, \\
1.162\end{array}$ \\
\hline 2 & $\begin{array}{l}565- \\
69-5\end{array}$ & 3-Pentanone, 2-methyl- & $\begin{array}{l}\mathrm{C}_{6} \mathrm{H}_{12} \\
\mathrm{O}\end{array}$ & Ketone & $\begin{array}{c}759.9 \\
42, \\
1.188\end{array}$ \\
\hline 2 & $\begin{array}{l}565- \\
80-0\end{array}$ & 3-Pentanone, 2,4-dimethyl- & $\begin{array}{l}\mathrm{C}_{7} \mathrm{H}_{14} \\
\quad \mathrm{O}\end{array}$ & Ketone & $\begin{array}{c}827.9 \\
35, \\
1.135\end{array}$ \\
\hline 2 & $\begin{array}{c}1252 \\
84- \\
20-0\end{array}$ & $\begin{array}{l}\text { 4-(1-Hydroperoxy-2,2-dimethyl-6-methylene- } \\
\text { cyclohexyl)-pent-3-en-2-one }\end{array}$ & $\begin{array}{c}\mathrm{C}_{14} \\
\mathrm{H}_{22} \mathrm{O}_{3}\end{array}$ & Ketone & $\begin{array}{c}1803 . \\
84, \\
2.237\end{array}$ \\
\hline 2 & $\begin{array}{c}3796 \\
-70- \\
1\end{array}$ & 5,9-Undecadien-2-one, 6,10-dimethyl-, (E)- & $\begin{array}{c}\mathrm{C}_{13} \\
\mathrm{H}_{22} \mathrm{O}\end{array}$ & Ketone & $\begin{array}{c}1763 . \\
84, \\
1.683\end{array}$ \\
\hline 2 & $\begin{array}{c}7481 \\
0- \\
53-0\end{array}$ & 6-Octen-2-one, (Z)- & $\begin{array}{l}\mathrm{C}_{8} \mathrm{H}_{14} \\
\mathrm{O}\end{array}$ & Ketone & $\begin{array}{c}1071 . \\
91, \\
1.267\end{array}$ \\
\hline 2 & $\begin{array}{c}5239 \\
8- \\
48-8\end{array}$ & $\begin{array}{l}\text { Ò- , - -Dimethyl-Á-(hydroxy-methyl)-Á- } \\
\text { butyrolactone }\end{array}$ & $\begin{array}{c}\mathrm{C}_{7} \mathrm{H}_{12} \\
\mathrm{O}_{3}\end{array}$ & Ketone & $\begin{array}{l}1243 . \\
89 \\
1.947\end{array}$ \\
\hline 1 & $\begin{array}{c}3068 \\
-88- \\
0\end{array}$ & -Butyrolactone & $\begin{array}{c}\mathrm{C}_{4} \mathrm{H}_{6} \\
\mathrm{O}_{2}\end{array}$ & Ketone & $\begin{array}{c}667.9 \\
51 \\
1.366\end{array}$ \\
\hline
\end{tabular}




\begin{tabular}{|c|c|c|c|c|c|}
\hline 1 & $\begin{array}{l}693- \\
54-9\end{array}$ & 2-Decanone & $\begin{array}{c}\mathrm{C}_{10} \\
\mathrm{H}_{20} \mathrm{O}\end{array}$ & Ketone & $\begin{array}{c}2163 . \\
8 \\
1.571\end{array}$ \\
\hline 1 & $\begin{array}{c}6175 \\
-49- \\
1\end{array}$ & 2-Dodecanone & $\begin{array}{c}\mathrm{C}_{12} \\
\mathrm{H}_{24} \mathrm{O}\end{array}$ & Ketone & $\begin{array}{c}2163 . \\
8 \\
1.577\end{array}$ \\
\hline 1 & $\begin{array}{l}928- \\
68-7\end{array}$ & 2-Heptanone, 6-methyl- & $\begin{array}{c}\mathrm{C}_{8} \mathrm{H}_{16} \\
\mathrm{O}\end{array}$ & Ketone & $\begin{array}{c}1039 . \\
91 \\
1.188\end{array}$ \\
\hline 1 & $\begin{array}{c}2231 \\
9- \\
24-0\end{array}$ & 2-Hepten-4-one, 2-methyl- & $\begin{array}{c}\mathrm{C}_{8} \mathrm{H}_{14} \\
\mathrm{O}\end{array}$ & Ketone & $\begin{array}{c}1027 . \\
92 \\
1.175\end{array}$ \\
\hline 1 & $\begin{array}{l}105- \\
42-0\end{array}$ & 2-Hexanone, 4-methyl- & $\begin{array}{c}\mathrm{C}_{7} \mathrm{H}_{14} \\
\mathrm{O}\end{array}$ & Ketone & $\begin{array}{c}959.9 \\
22, \\
1.201\end{array}$ \\
\hline 1 & $\begin{array}{c}5704 \\
-20- \\
1\end{array}$ & 2-Hydroxy-3-pentanone & $\begin{array}{c}\mathrm{C}_{5} \mathrm{H}_{10} \\
\mathrm{O}_{2}\end{array}$ & Ketone & $\begin{array}{c}843.9 \\
34, \\
1.940\end{array}$ \\
\hline 1 & $\begin{array}{l}629- \\
66-3\end{array}$ & 2-Nonadecanone & $\begin{array}{c}\mathrm{C}_{19} \\
\mathrm{H}_{38} \mathrm{O}\end{array}$ & Ketone & $\begin{array}{c}2163 . \\
8 \\
1.571\end{array}$ \\
\hline 1 & $\begin{array}{c}4359 \\
-77- \\
7\end{array}$ & 2-Pentanone, 3-methylene- & $\begin{array}{c}\mathrm{C}_{6} \mathrm{H}_{10} \\
\mathrm{O}\end{array}$ & Ketone & $\begin{array}{c}839.9 \\
34, \\
1.313\end{array}$ \\
\hline 1 & $\begin{array}{l}123- \\
42-2\end{array}$ & 2-Pentanone, 4-hydroxy-4-methyl- & $\begin{array}{c}\mathrm{C}_{6} \mathrm{H}_{12} \\
\mathrm{O}_{2}\end{array}$ & Ketone & $\begin{array}{c}899.9 \\
28, \\
1.736\end{array}$ \\
\hline 1 & $\begin{array}{c}5878 \\
-19- \\
3\end{array}$ & 2-Propanone, 1-methoxy- & $\begin{array}{c}\mathrm{C}_{4} \mathrm{H}_{8} \\
\mathrm{O}_{2}\end{array}$ & Ketone & $\begin{array}{c}691.9 \\
49 \\
2.475\end{array}$ \\
\hline 1 & $\begin{array}{c}1907 \\
8- \\
97-8\end{array}$ & 2,2-Dimethyl-3-heptanone & $\begin{array}{c}\mathrm{C}_{9} \mathrm{H}_{18} \\
\mathrm{O}\end{array}$ & Ketone & $\begin{array}{c}1259 . \\
89 \\
1.861\end{array}$ \\
\hline 1 & $\begin{array}{c}1575 \\
-46- \\
8\end{array}$ & 2,3-Dimethyl-4-hydroxy-2-butenoic lactone & $\begin{array}{c}\mathrm{C}_{6} \mathrm{H}_{8} \\
\mathrm{O}_{2}\end{array}$ & Ketone & $\begin{array}{c}1327 . \\
89 \\
3.749\end{array}$ \\
\hline 1 & $\begin{array}{l}600- \\
14-6\end{array}$ & 2,3-Pentanedione & $\begin{array}{c}\mathrm{C}_{5} \mathrm{H}_{8} \\
\mathrm{O}_{2}\end{array}$ & Ketone & $\begin{array}{c}1075 . \\
91 \\
1.168\end{array}$ \\
\hline 1 & $\begin{array}{c}79- \\
77-6\end{array}$ & $\begin{array}{l}\text { 3-Buten-2-one, 4-(2,6,6-trimethyl-1-cyclohexen-1- } \\
\text { yl)-, (E)- }\end{array}$ & $\begin{array}{c}\mathrm{C}_{13} \\
\mathrm{H}_{20} \mathrm{O}\end{array}$ & Ketone & $\begin{array}{c}1855 . \\
83 \\
1.874\end{array}$ \\
\hline 1 & $\begin{array}{l}541- \\
85-5\end{array}$ & 3-Heptanone, 5-methyl- & $\begin{array}{c}\mathrm{C}_{8} \mathrm{H}_{16} \\
\mathrm{O}\end{array}$ & Ketone & $\begin{array}{c}1075 . \\
91 \\
1.188\end{array}$ \\
\hline 1 & $\begin{array}{c}2069 \\
0- \\
70-4\end{array}$ & 3-Heptanone, 5-methylene- & $\begin{array}{l}\mathrm{C}_{8} \mathrm{H}_{14} \\
\mathrm{O}\end{array}$ & Ketone & $\begin{array}{c}1079 . \\
91 \\
1.241\end{array}$ \\
\hline 1 & $\begin{array}{c}1864 \\
1- \\
70-8\end{array}$ & 3-Hexanone, 2,4-dimethyl- & $\begin{array}{c}\mathrm{C}_{8} \mathrm{H}_{16} \\
\mathrm{O}\end{array}$ & Ketone & $\begin{array}{c}1075 . \\
91 \\
1.175\end{array}$ \\
\hline 1 & $923-$ & 3-Octanone, 2-methyl- & $\mathrm{C}_{9} \mathrm{H}_{18}$ & Ketone & 1211. \\
\hline
\end{tabular}




\begin{tabular}{|c|c|c|c|c|c|}
\hline & $28-4$ & & $\mathrm{O}$ & & $\begin{array}{c}9, \\
1.934\end{array}$ \\
\hline 1 & $\begin{array}{c}1664 \\
7- \\
04-4\end{array}$ & 3,5-Heptadien-2-one, 6-methyl-, (E)- & $\begin{array}{l}\mathrm{C}_{8} \mathrm{H}_{12} \\
\mathrm{O}\end{array}$ & Ketone & $\begin{array}{l}979.9 \\
2 \\
1.135\end{array}$ \\
\hline 1 & $\begin{array}{c}2517 \\
2- \\
06-9\end{array}$ & 3,7-Octadien-2-one, (E)- & $\begin{array}{l}\mathrm{C}_{8} \mathrm{H}_{12} \\
\mathrm{O}\end{array}$ & Ketone & $\begin{array}{c}1067 . \\
91 \\
1.346\end{array}$ \\
\hline 1 & $\begin{array}{c}1412 \\
9- \\
48-7\end{array}$ & 4-Octen-3-one & $\begin{array}{c}\mathrm{C}_{8} \mathrm{H}_{14} \\
\mathrm{O}\end{array}$ & Ketone & $\begin{array}{l}1131 . \\
9 \\
1.307\end{array}$ \\
\hline 1 & $\begin{array}{l}110- \\
93-0\end{array}$ & 5-Hepten-2-one, 6-methyl- & $\begin{array}{c}\mathrm{C}_{8} \mathrm{H}_{14} \\
\mathrm{O}\end{array}$ & Ketone & $\begin{array}{c}1075 . \\
91 \\
1.254\end{array}$ \\
\hline 1 & $\begin{array}{l}109- \\
49-9\end{array}$ & 5-Hexen-2-one & $\begin{array}{c}\mathrm{C}_{6} \mathrm{H}_{10} \\
\mathrm{O}\end{array}$ & Ketone & $\begin{array}{c}1071 . \\
91 \\
1.247\end{array}$ \\
\hline 1 & $\begin{array}{c}6233 \\
8- \\
59-4\end{array}$ & 6-Hepten-3-one, 5-hydroxy-4,6-dimethyl- & $\begin{array}{c}\mathrm{C}_{9} \mathrm{H}_{16} \\
\mathrm{O}_{2}\end{array}$ & Ketone & $\begin{array}{c}1087 . \\
91 \\
1.129\end{array}$ \\
\hline 1 & $\begin{array}{c}1604 \\
-28- \\
0\end{array}$ & 6-Methyl-3,5-heptadiene-2-one & $\begin{array}{l}\mathrm{C}_{8} \mathrm{H}_{12} \\
\mathrm{O}\end{array}$ & Ketone & $\begin{array}{c}947.9 \\
23 \\
1.109\end{array}$ \\
\hline 1 & $\begin{array}{c}3664 \\
-60- \\
6\end{array}$ & 7-Octen-2-one & $\begin{array}{c}\mathrm{C}_{8} \mathrm{H}_{14} \\
\mathrm{O}\end{array}$ & Ketone & $\begin{array}{l}979.9 \\
2 \\
1.096\end{array}$ \\
\hline 1 & $\begin{array}{c}0- \\
00-0\end{array}$ & $\begin{array}{c}\text { Benzofran-3-one, 2-[3,4-dihydroxybenzylidene]-6- } \\
\text { hydroxy- }\end{array}$ & $\begin{array}{c}\mathrm{C}_{15} \\
\mathrm{H}_{10} \mathrm{O}_{5}\end{array}$ & Ketone & $\begin{array}{c}2603 . \\
76, \\
1.749\end{array}$ \\
\hline 1 & $\begin{array}{l}108- \\
10-1\end{array}$ & Methyl Isobutyl Ketone & $\begin{array}{c}\mathrm{C}_{6} \mathrm{H}_{12} \\
\mathrm{O}\end{array}$ & Ketone & $\begin{array}{c}747.9 \\
43, \\
1.208\end{array}$ \\
\hline 1 & $\begin{array}{c}5340 \\
-30- \\
7\end{array}$ & Neopentyl ethyl ketone & $\begin{array}{c}\mathrm{C}_{8} \mathrm{H}_{16} \\
\mathrm{O}\end{array}$ & Ketone & $\begin{array}{c}1087 . \\
91 \\
1.162\end{array}$ \\
\hline 16 & $\begin{array}{c}0- \\
00-0\end{array}$ & 3-Ethyl-1,5-octadiene & $\begin{array}{l}\mathrm{C}_{10} \\
\mathrm{H}_{18}\end{array}$ & $\begin{array}{l}\text { Monote } \\
\text { rpene }\end{array}$ & $\begin{array}{c}1011 . \\
92, \\
1.016\end{array}$ \\
\hline 5 & $\begin{array}{c}5989 \\
-54- \\
8\end{array}$ & Cyclohexene, 1-methyl-4-(1-methylethenyl)-, (S)- & $\begin{array}{l}\mathrm{C}_{10} \\
\mathrm{H}_{16}\end{array}$ & $\begin{array}{l}\text { Monote } \\
\text { rpene }\end{array}$ & $\begin{array}{c}1139 . \\
9 \\
1.135\end{array}$ \\
\hline 2 & $\begin{array}{c}99- \\
83-2\end{array}$ & ‡-Phellandrene & $\begin{array}{l}\mathrm{C}_{10} \\
\mathrm{H}_{16}\end{array}$ & $\begin{array}{l}\text { Monote } \\
\text { rpene }\end{array}$ & $\begin{array}{c}1107 . \\
91 \\
1.115\end{array}$ \\
\hline 1 & $\begin{array}{c}6384 \\
3- \\
03-8\end{array}$ & 2,6-Octadiene, 2,4-dimethyl- & $\begin{array}{l}\mathrm{C}_{10} \\
\mathrm{H}_{18}\end{array}$ & $\begin{array}{l}\text { Monote } \\
\text { rpene }\end{array}$ & $\begin{array}{c}1675 . \\
85 \\
1.274\end{array}$ \\
\hline 1 & $\begin{array}{l}138- \\
86-3\end{array}$ & Limonene & $\begin{array}{l}\mathrm{C}_{10} \\
\mathrm{H}_{16}\end{array}$ & $\begin{array}{l}\text { Monote } \\
\text { rpene }\end{array}$ & $\begin{array}{c}1139 . \\
9, \\
1.135\end{array}$ \\
\hline 11 & $\begin{array}{c}95- \\
16-9\end{array}$ & Benzothiazole & $\begin{array}{c}\mathrm{C}_{7} \mathrm{H}_{5} \\
\mathrm{NS}\end{array}$ & Nitro & $\begin{array}{c}1455 . \\
87\end{array}$ \\
\hline
\end{tabular}




\begin{tabular}{|c|c|c|c|c|c|}
\hline & & & & & 3.003 \\
\hline 7 & $\begin{array}{c}0- \\
00-0\end{array}$ & Oxime-, methoxy-phenyl-_ & $\begin{array}{c}\mathrm{C}_{8} \mathrm{H}_{9} \\
\mathrm{NO}_{2}\end{array}$ & Nitro & $\begin{array}{c}927.9 \\
25, \\
3.511\end{array}$ \\
\hline 5 & $\begin{array}{l}761- \\
65-9\end{array}$ & Formamide, N,N-dibutyl- & $\begin{array}{c}\mathrm{C}_{9} \mathrm{H}_{19} \\
\mathrm{NO}\end{array}$ & Nitro & $\begin{array}{c}1539 . \\
86, \\
1.868\end{array}$ \\
\hline 4 & $\begin{array}{c}1467 \\
24- \\
75-6\end{array}$ & 2,5-Dimethoxy-4-(methylsulfonyl)amphetamine & $\begin{array}{c}\mathrm{C}_{12} \\
\mathrm{H}_{19} \\
\mathrm{NO}_{4} \mathrm{~S}\end{array}$ & Nitro & $\begin{array}{c}283.9 \\
9 \\
0.970\end{array}$ \\
\hline 4 & $\begin{array}{l}584- \\
84-9\end{array}$ & Benzene, 2,4-diisocyanato-1-methyl- & $\begin{array}{l}\mathrm{C}_{9} \mathrm{H}_{6} \\
\mathrm{~N}_{2} \mathrm{O}_{2}\end{array}$ & Nitro & $\begin{array}{c}1639 . \\
85 \\
2.145\end{array}$ \\
\hline 3 & $\begin{array}{c}3013 \\
17- \\
49-7\end{array}$ & $\begin{array}{l}\text { 1-[6-Hydroxy-4-(4-hydroxy-3-methoxy-phenyl)- } \\
\text { 3,6-dimethyl-4,5,6,7-tetrahydro-2H-indazol-5-yl]- } \\
\text { ethanone }\end{array}$ & $\begin{array}{c}\mathrm{C}_{18} \\
\mathrm{H}_{22} \\
\mathrm{~N}_{2} \mathrm{O}_{4}\end{array}$ & Nitro & $\begin{array}{c}2283 . \\
79 \\
1.742\end{array}$ \\
\hline 3 & $\begin{array}{l}100- \\
45-8\end{array}$ & 4-Cyanocyclohexene & $\begin{array}{c}\mathrm{C}_{7} \mathrm{H}_{9} \\
\mathrm{~N}\end{array}$ & Nitro & $\begin{array}{c}1127 . \\
91, \\
1.855\end{array}$ \\
\hline 3 & $\begin{array}{c}2182 \\
3- \\
29-0\end{array}$ & Cyclopentanol, nitrate & $\begin{array}{c}\mathrm{C}_{5} \mathrm{H}_{9} \\
\mathrm{NO}_{3}\end{array}$ & Nitro & $\begin{array}{c}895.9 \\
28, \\
1.234\end{array}$ \\
\hline 2 & $\begin{array}{c}0- \\
00-0\end{array}$ & 8-Methyl-4-azafluorene, phenylimine & $\begin{array}{l}\mathrm{C}_{19} \\
\mathrm{H}_{14} \\
\mathrm{~N}_{2}\end{array}$ & Nitro & $\begin{array}{c}2631 . \\
75 \\
1.742\end{array}$ \\
\hline 2 & $\begin{array}{l}100- \\
47-0\end{array}$ & Benzonitrile & $\begin{array}{c}\mathrm{C}_{7} \mathrm{H}_{5} \\
\mathrm{~N}\end{array}$ & Nitro & $\begin{array}{c}1091 . \\
91 \\
1.980\end{array}$ \\
\hline 2 & $\begin{array}{c}2562 \\
-38- \\
1\end{array}$ & Cyclopentane, nitro- & $\begin{array}{c}\mathrm{C}_{5} \mathrm{H}_{9} \\
\mathrm{NO}_{2}\end{array}$ & Nitro & $\begin{array}{c}1099 . \\
91, \\
1.069\end{array}$ \\
\hline 2 & $\begin{array}{c}7742 \\
2- \\
34-5\end{array}$ & N-Morpholinomethyl-isopropyl-sulfide & $\begin{array}{c}\mathrm{C}_{8} \mathrm{H}_{17} \\
\text { NOS }\end{array}$ & Nitro & $\begin{array}{c}1795 . \\
84 \\
1.472\end{array}$ \\
\hline 2 & $\begin{array}{l}540- \\
43-2\end{array}$ & N,1-Dimethylhexylamine & $\begin{array}{c}\mathrm{C}_{8} \mathrm{H}_{19} \\
\mathrm{~N}\end{array}$ & Nitro & $\begin{array}{c}1835 . \\
83 \\
1.195\end{array}$ \\
\hline 1 & $\begin{array}{c}1382 \\
2- \\
06-5\end{array}$ & 1-Amino-3-methyl-2-butene & $\begin{array}{c}\mathrm{C}_{5} \mathrm{H}_{11} \\
\mathrm{~N}\end{array}$ & Nitro & $\begin{array}{c}667.9 \\
51, \\
1.340\end{array}$ \\
\hline 1 & $\begin{array}{c}1120 \\
-24- \\
7\end{array}$ & 1-Decanamine, N,N-dimethyl- & $\begin{array}{c}\mathrm{C}_{12} \\
\mathrm{H}_{27} \mathrm{~N}\end{array}$ & Nitro & $\begin{array}{c}2543 . \\
76, \\
1.505\end{array}$ \\
\hline 1 & $\begin{array}{c}1737 \\
3- \\
27-2\end{array}$ & 1-Nonanamine, N,N-dimethyl- & $\begin{array}{c}\mathrm{C}_{11} \\
\mathrm{H}_{25} \mathrm{~N}\end{array}$ & Nitro & $\begin{array}{c}2535 . \\
76, \\
1.445\end{array}$ \\
\hline 1 & $\begin{array}{c}1767 \\
8- \\
60-3\end{array}$ & 1-Pentadecanamine, N,N-dimethyl- & $\begin{array}{c}\mathrm{C}_{17} \mathrm{H}_{3} \\
7 \mathrm{~N}\end{array}$ & Nitro & $\begin{array}{c}2159 . \\
8 \\
1.234\end{array}$ \\
\hline 1 & $\begin{array}{c}1737 \\
3- \\
29-4\end{array}$ & 1-Tridecanamine, N,N-dimethyl- & $\begin{array}{c}\mathrm{C}_{15} \mathrm{H}_{3} \\
{ }_{3} \mathrm{~N}\end{array}$ & Nitro & $\begin{array}{c}2543 . \\
76, \\
1.452\end{array}$ \\
\hline
\end{tabular}




\begin{tabular}{|c|c|c|c|c|c|}
\hline 1 & $\begin{array}{c}1737 \\
3- \\
28-3\end{array}$ & 1-Undecanamine, N,N-dimethyl- & $\begin{array}{c}\mathrm{C}_{13} \\
\mathrm{H}_{29} \mathrm{~N}\end{array}$ & Nitro & $\begin{array}{c}2543 . \\
76, \\
1.505\end{array}$ \\
\hline 1 & $\begin{array}{c}78- \\
90-0\end{array}$ & 1,2-Propanediamine & $\begin{array}{c}\mathrm{C}_{3} \mathrm{H}_{10} \\
\mathrm{~N}_{2}\end{array}$ & Nitro & $\begin{array}{c}339.9 \\
84, \\
0.957\end{array}$ \\
\hline 1 & $\begin{array}{c}0- \\
00-0\end{array}$ & $\begin{array}{l}\text { 1,4-Methano-1H-cyclohepta[d]pyridazine, } \\
\text { 4,4a,5,6,7,8,9,9a-octahydro-10,10-dimethyl- }\end{array}$ & $\begin{array}{c}\mathrm{C}_{12} \\
\mathrm{H}_{20} \\
\mathrm{~N}_{2}\end{array}$ & Nitro & $\begin{array}{c}1459 . \\
87 \\
1.313\end{array}$ \\
\hline 1 & $\begin{array}{c}1668 \\
1- \\
77-9\end{array}$ & 1H-Tetrazole, 1-methyl- & $\begin{array}{c}\mathrm{C}_{2} \mathrm{H}_{4} \\
\mathrm{~N}_{4}\end{array}$ & Nitro & $\begin{array}{c}1087 . \\
91 \\
0.997\end{array}$ \\
\hline 1 & $\begin{array}{l}764- \\
42-1\end{array}$ & 2-Butenedinitrile, (E)- & $\begin{array}{c}\mathrm{C}_{4} \mathrm{H}_{2} \\
\mathrm{~N}_{2}\end{array}$ & Nitro & $\begin{array}{c}667.9 \\
51, \\
1.228\end{array}$ \\
\hline 1 & $\begin{array}{l}811- \\
93-8\end{array}$ & 2-Methyl-1,2-propanediamine & $\begin{array}{c}\mathrm{C}_{4} \mathrm{H}_{12} \\
\mathrm{~N}_{2}\end{array}$ & Nitro & $\begin{array}{c}2539 . \\
76 \\
1.505\end{array}$ \\
\hline 1 & $\begin{array}{c}1951 \\
94- \\
80-0\end{array}$ & 2-Piperidinone, N-[4-bromo-n-butyl]- & $\begin{array}{l}\mathrm{C}_{9} \mathrm{H}_{16} \\
\mathrm{BrNO}\end{array}$ & Nitro & $\begin{array}{c}2051 . \\
81 \\
1.366\end{array}$ \\
\hline 1 & $\begin{array}{c}75- \\
31-0\end{array}$ & 2-Propanamine & $\begin{array}{c}\mathrm{C}_{3} \mathrm{H}_{9} \\
\mathrm{~N}\end{array}$ & Nitro & $\begin{array}{c}343.9 \\
84, \\
0.957\end{array}$ \\
\hline 1 & $\begin{array}{c}1543 \\
1- \\
05-7\end{array}$ & 3-Buten-1-amine, N,N-diethyl- & $\begin{array}{l}\mathrm{C}_{8} \mathrm{H}_{17} \\
\quad \mathrm{~N}\end{array}$ & Nitro & $\begin{array}{c}667.9 \\
51, \\
1.280\end{array}$ \\
\hline 1 & $\begin{array}{c}1590 \\
1- \\
42-5\end{array}$ & 3,3,5-Trimethylcyclohexylamine & $\begin{array}{l}\mathrm{C}_{9} \mathrm{H}_{19} \\
\quad \mathrm{~N}\end{array}$ & Nitro & $\begin{array}{c}667.9 \\
51, \\
1.313\end{array}$ \\
\hline 1 & $\begin{array}{c}1332 \\
5- \\
10-5\end{array}$ & 4-Amino-1-butanol & $\begin{array}{c}\mathrm{C}_{4} \mathrm{H}_{11} \\
\mathrm{NO}\end{array}$ & Nitro & $\begin{array}{c}1215 . \\
9 \\
1.914\end{array}$ \\
\hline 1 & $\begin{array}{l}693- \\
95-8\end{array}$ & 4-Methylthiazole & $\begin{array}{l}\mathrm{C}_{4} \mathrm{H}_{5} \\
\mathrm{NS}\end{array}$ & Nitro & $\begin{array}{c}1075 . \\
91 \\
1.195\end{array}$ \\
\hline 1 & $\begin{array}{c}7628 \\
4- \\
12-3\end{array}$ & 5-Pyrrolidino-2-pyrrolidone & $\begin{array}{c}\mathrm{C}_{8} \mathrm{H}_{14} \\
\mathrm{~N}_{2} \mathrm{O}\end{array}$ & Nitro & $\begin{array}{c}2151 . \\
8 \\
1.155\end{array}$ \\
\hline 1 & $\begin{array}{c}0- \\
00-0\end{array}$ & 5H-Tetrazol-5-amine & $\begin{array}{l}\mathrm{CH}_{3} \\
\mathrm{~N} 5\end{array}$ & Nitro & $\begin{array}{c}1035 . \\
91, \\
1.855\end{array}$ \\
\hline 1 & $\begin{array}{c}0- \\
00-0\end{array}$ & $\begin{array}{l}\text { 6-Amino-5-cyano-4-isobutyl-2-phenyl-4H-pyran- } \\
\text { 3-carboxylic acid ethyl ester }\end{array}$ & $\begin{array}{l}\mathrm{C}_{19} \\
\mathrm{H}_{22} \\
\mathrm{~N}_{2} \mathrm{O}_{3}\end{array}$ & Nitro & $\begin{array}{c}2323 . \\
79 \\
1.742\end{array}$ \\
\hline 1 & $\begin{array}{c}3378 \\
8- \\
00-0\end{array}$ & 6-Undecylamine & $\begin{array}{c}\mathrm{C}_{11} \\
\mathrm{H}_{25} \mathrm{~N}\end{array}$ & Nitro & $\begin{array}{c}2531 . \\
76 \\
1.921\end{array}$ \\
\hline 1 & $\begin{array}{l}101- \\
40-6\end{array}$ & Benzedrex & $\begin{array}{l}\mathrm{C}_{10} \mathrm{H}_{2} \\
1 \mathrm{~N}\end{array}$ & Nitro & $\begin{array}{l}2159 . \\
8 \\
1.234\end{array}$ \\
\hline 1 & 5279 & Benzene, (1-nitropropyl)- & $\mathrm{C}_{9} \mathrm{H}_{11}$ & Nitro & 2123. \\
\hline
\end{tabular}




\begin{tabular}{|c|c|c|c|c|c|}
\hline & $\begin{array}{c}-14- \\
1\end{array}$ & & $\mathrm{NO}_{2}$ & & $\begin{array}{c}81 \\
1.419\end{array}$ \\
\hline 1 & $\begin{array}{c}91- \\
08-7\end{array}$ & Benzene, 1,3-diisocyanato-2-methyl- & $\begin{array}{l}\mathrm{C}_{9} \mathrm{H}_{6} \\
\mathrm{~N}_{2} \mathrm{O}_{2}\end{array}$ & Nitro & $\begin{array}{c}1647 . \\
85 \\
2.132\end{array}$ \\
\hline 1 & $\begin{array}{c}1283 \\
53- \\
59-3\end{array}$ & $\begin{array}{l}\text { Boron, (N,2-dimethyl-2- } \\
\text { propanamine)tris(trifluoromethyl)-, (t-4)- }\end{array}$ & $\begin{array}{l}\mathrm{C}_{8} \mathrm{H}_{13} \\
\mathrm{BF}_{9} \mathrm{~N}\end{array}$ & Nitro & $\begin{array}{l}1087 . \\
91 \\
1.175\end{array}$ \\
\hline 1 & $\begin{array}{c}7460 \\
9- \\
73-7\end{array}$ & cis-2-Nitro-4-t-butylcyclohexanone & $\begin{array}{c}\mathrm{C}_{10} \\
\mathrm{H}_{17} \\
\mathrm{NO}_{3}\end{array}$ & Nitro & $\begin{array}{c}1075 . \\
91 \\
1.261\end{array}$ \\
\hline 1 & $\begin{array}{l}927- \\
83-3\end{array}$ & Diazene, bis(1,1-dimethylethyl)- & $\begin{array}{c}\mathrm{C}_{8} \mathrm{H}_{18} \\
\mathrm{~N}_{2}\end{array}$ & Nitro & $\begin{array}{c}1067 . \\
91 \\
1.346\end{array}$ \\
\hline 1 & $\begin{array}{c}6247 \\
4- \\
28-6\end{array}$ & $\begin{array}{l}\text { Dimethylamine, } \mathrm{N} \text { - } \\
\text { (diisopropylphosphino)methyl- }\end{array}$ & $\begin{array}{l}\mathrm{C}_{9} \mathrm{H}_{22} \\
\mathrm{NP}\end{array}$ & Nitro & $\begin{array}{l}2543 . \\
76 \\
1.452\end{array}$ \\
\hline 1 & $\begin{array}{c}4218 \\
5- \\
03-5\end{array}$ & Ethanamine, 2-propoxy- & $\begin{array}{l}\mathrm{C}_{5} \mathrm{H}_{13} \\
\mathrm{NO}\end{array}$ & Nitro & $\begin{array}{c}823.9 \\
36 \\
1.208\end{array}$ \\
\hline 1 & $\begin{array}{l}625- \\
48-9\end{array}$ & Ethanol, 2-nitro- & $\begin{array}{l}\mathrm{C}_{2} \mathrm{H}_{5} \\
\mathrm{NO}_{3}\end{array}$ & Nitro & $\begin{array}{l}275.9 \\
9 \\
2.587\end{array}$ \\
\hline 1 & $\begin{array}{c}7177 \\
3- \\
95-0\end{array}$ & l-Alanine ethylamide, (S)- & $\begin{array}{l}\mathrm{C}_{5} \mathrm{H}_{12} \\
\mathrm{~N}_{2} \mathrm{O}\end{array}$ & Nitro & $\begin{array}{l}347.9 \\
83 \\
0.957\end{array}$ \\
\hline 1 & $\begin{array}{l}1113 \\
-41- \\
3\end{array}$ & L-Valine, 3-mercapto- & $\begin{array}{l}\mathrm{C}_{5} \mathrm{H}_{11} \\
\mathrm{NO}_{2} \mathrm{~S}\end{array}$ & Nitro & $\begin{array}{c}1087 . \\
91 \\
1.135\end{array}$ \\
\hline 1 & $\begin{array}{c}1527 \\
-89- \\
5\end{array}$ & m-Methoxybenzontrile & $\begin{array}{l}\mathrm{C}_{8} \mathrm{H}_{7} \\
\mathrm{NO}\end{array}$ & Nitro & $\begin{array}{c}1095 . \\
91 \\
1.980\end{array}$ \\
\hline 1 & $\begin{array}{c}5559 \\
0- \\
26-6\end{array}$ & N-Methyl-7-azabicyclo $(2,2,1)$ hept-2-ene & $\begin{array}{l}\mathrm{C}_{7} \mathrm{H}_{11} \\
\mathrm{~N}\end{array}$ & Nitro & $\begin{array}{l}315.9 \\
86 \\
1.043\end{array}$ \\
\hline 1 & $\begin{array}{c}4774 \\
-33- \\
8\end{array}$ & N,N',N"-Methylidynetrisformamide & $\begin{array}{l}\mathrm{C}_{4} \mathrm{H}_{7} \\
\mathrm{~N}_{3} \mathrm{O}_{3}\end{array}$ & Nitro & $\begin{array}{l}835.9 \\
34 \\
1.208\end{array}$ \\
\hline 1 & $\begin{array}{l}108- \\
03-2\end{array}$ & Propane, 1-nitro- & $\begin{array}{l}\mathrm{C}_{3} \mathrm{H}_{7} \\
\mathrm{NO}_{2}\end{array}$ & Nitro & $\begin{array}{c}1075 . \\
91 \\
1.313\end{array}$ \\
\hline 1 & $\begin{array}{c}1034 \\
95- \\
51-8\end{array}$ & Tricyclo[3.1.0.0(2,4)]hex-3-ene-3-carbonitrile & $\begin{array}{l}\mathrm{C}_{7} \mathrm{H}_{5} \\
\mathrm{~N}\end{array}$ & Nitro & $\begin{array}{c}1095 . \\
91 \\
1.954\end{array}$ \\
\hline 1 & $\begin{array}{l}123- \\
82-0\end{array}$ & Tuaminoheptane & $\begin{array}{l}\mathrm{C}_{7} \mathrm{H}_{17} \\
\mathrm{~N}\end{array}$ & Nitro & $\begin{array}{l}315.9 \\
86 \\
0.964\end{array}$ \\
\hline 1 & $\begin{array}{c}0- \\
00-0\end{array}$ & $\begin{array}{l}\text { Undecanoic acid isopropyl ester, 10-hydroxy-11- } \\
\text { morpholin-4-yl- }\end{array}$ & $\begin{array}{l}\mathrm{C}_{18} \\
\mathrm{H}_{35} \\
\mathrm{NO}_{4}\end{array}$ & Nitro & $\begin{array}{c}2535 . \\
76 \\
1.894\end{array}$ \\
\hline 5 & $\begin{array}{l}527- \\
35-5\end{array}$ & Phenol, 2,3,5,6-tetramethyl- & $\begin{array}{c}\mathrm{C}_{10} \\
\mathrm{H}_{14} \mathrm{O} \\
\end{array}$ & $\begin{array}{l}\text { Oxy.Be } \\
\text { nzene }\end{array}$ & $\begin{array}{c}1251 . \\
89,\end{array}$ \\
\hline
\end{tabular}




\begin{tabular}{|c|c|c|c|c|c|}
\hline & & & & & 1.399 \\
\hline 5 & $\begin{array}{l}527- \\
60-6\end{array}$ & Phenol, 2,4,6-trimethyl- & $\begin{array}{c}\mathrm{C}_{9} \mathrm{H}_{12} \\
\mathrm{O}\end{array}$ & $\begin{array}{c}\text { Oxy.Be } \\
\text { nzene }\end{array}$ & $\begin{array}{c}1387 . \\
88 \\
3.241\end{array}$ \\
\hline 2 & $\begin{array}{l}122- \\
03-2\end{array}$ & Benzaldehyde, 4-(1-methylethyl)- & $\begin{array}{c}\mathrm{C}_{10} \\
\mathrm{H}_{12} \mathrm{O}\end{array}$ & $\begin{array}{c}\text { Oxy.Be } \\
\text { nzene }\end{array}$ & $\begin{array}{c}791.9 \\
39, \\
1.340\end{array}$ \\
\hline 1 & $\begin{array}{l}122- \\
78-1\end{array}$ & Benzeneacetaldehyde & $\begin{array}{c}\mathrm{C}_{8} \mathrm{H}_{8} \\
\mathrm{O}\end{array}$ & $\begin{array}{c}\text { Oxy.Be } \\
\text { nzene }\end{array}$ & $\begin{array}{c}1163 . \\
9 \\
2.033\end{array}$ \\
\hline 1 & $\begin{array}{c}4814 \\
1- \\
64-6\end{array}$ & $\begin{array}{l}\text { Benzenemethanol, } \$-[1- \\
\text { (ethylmethylamino)ethyl]-, }\left[\mathrm{R}-\left(\mathrm{R}^{*}, \mathrm{~S}^{*}\right)\right]-\end{array}$ & $\begin{array}{l}\mathrm{C}_{12} \\
\mathrm{H}_{19} \\
\mathrm{NO}\end{array}$ & $\begin{array}{l}\text { Oxy.Be } \\
\text { nzene }\end{array}$ & $\begin{array}{c}667.9 \\
51 \\
1.287\end{array}$ \\
\hline 1 & $\begin{array}{l}536- \\
60-7\end{array}$ & Benzenemethanol, 4-(1-methylethyl)- & $\begin{array}{c}\mathrm{C}_{10} \\
\mathrm{H}_{14} \mathrm{O}\end{array}$ & $\begin{array}{c}\text { Oxy.Be } \\
\text { nzene }\end{array}$ & $\begin{array}{c}1251 . \\
89 \\
1.393\end{array}$ \\
\hline 1 & $\begin{array}{c}2219 \\
-78- \\
5\end{array}$ & Phenol, 2-ethyl-4,5-dimethyl- & $\begin{array}{c}\mathrm{C}_{10} \\
\mathrm{H}_{14} \mathrm{O}\end{array}$ & $\begin{array}{c}\text { Oxy.Be } \\
\text { nzene }\end{array}$ & $\begin{array}{c}1251 . \\
89 \\
1.399\end{array}$ \\
\hline 1 & $\begin{array}{l}370- \\
98-9\end{array}$ & Phenol, 4-[2-(methylamino)ethyl]- & $\begin{array}{l}\mathrm{C}_{9} \mathrm{H}_{13} \\
\mathrm{NO}\end{array}$ & $\begin{array}{l}\text { Oxy.Be } \\
\text { nzene }\end{array}$ & $\begin{array}{l}180 \\
3.577\end{array}$ \\
\hline 1 & $\begin{array}{c}5613 \\
-46- \\
7\end{array}$ & $\begin{array}{c}\text { Phenol, 4,4'-(1-methylethylidene)bis }[2,6 \text { - } \\
\text { dimethyl- }\end{array}$ & $\begin{array}{c}\mathrm{C}_{19} \\
\mathrm{H}_{24} \mathrm{O}_{2}\end{array}$ & $\begin{array}{l}\text { Oxy.Be } \\
\text { nzene }\end{array}$ & $\begin{array}{c}2131 . \\
8 \\
1.795\end{array}$ \\
\hline 20 & $\begin{array}{l}432- \\
25-7\end{array}$ & 1-Cyclohexene-1-carboxaldehyde, 2,6,6-trimethyl- & $\begin{array}{c}\mathrm{C}_{10} \\
\mathrm{H}_{16} \mathrm{O}\end{array}$ & $\begin{array}{c}\text { Oxy.Ri } \\
\text { ng }\end{array}$ & $\begin{array}{c}1427 . \\
88 \\
1.676\end{array}$ \\
\hline 6 & $\begin{array}{l}930- \\
37-0\end{array}$ & Oxirane, (methoxymethyl)- & $\begin{array}{c}\mathrm{C}_{4} \mathrm{H}_{8} \\
\mathrm{O}_{2}\end{array}$ & $\begin{array}{c}\text { Oxy.Ri } \\
\text { ng }\end{array}$ & $\begin{array}{c}843.9 \\
34, \\
1.967\end{array}$ \\
\hline 6 & $\begin{array}{c}1192 \\
-22- \\
9\end{array}$ & Oxirane, 3-ethyl-2,2-dimethyl- & $\begin{array}{c}\mathrm{C}_{6} \mathrm{H}_{12} \\
\mathrm{O}\end{array}$ & $\begin{array}{c}\text { Oxy.Ri } \\
\text { ng }\end{array}$ & $\begin{array}{c}699.9 \\
48, \\
1.122\end{array}$ \\
\hline 5 & $\begin{array}{c}3840 \\
1- \\
84-2\end{array}$ & 1,6-Dioxaspiro[4.4]nonane, 2-ethyl- & $\begin{array}{c}\mathrm{C}_{9} \mathrm{H}_{16} \\
\mathrm{O}_{2}\end{array}$ & $\begin{array}{c}\text { Oxy.Ri } \\
\text { ng }\end{array}$ & $\begin{array}{c}1199 . \\
9 \\
1.320\end{array}$ \\
\hline 4 & $\begin{array}{c}96- \\
48-0\end{array}$ & Butyrolactone & $\begin{array}{c}\mathrm{C}_{4} \mathrm{H}_{6} \\
\mathrm{O}_{2}\end{array}$ & $\begin{array}{c}\text { Oxy.Ri } \\
\text { ng }\end{array}$ & $\begin{array}{c}995.9 \\
18, \\
2.614\end{array}$ \\
\hline 4 & $\begin{array}{c}8918 \\
2- \\
08-1\end{array}$ & Cyclobut-1-enylmethanol & $\begin{array}{l}\mathrm{C}_{5} \mathrm{H}_{8} \\
\mathrm{O}\end{array}$ & $\begin{array}{c}\text { Oxy.Ri } \\
\text { ng }\end{array}$ & $\begin{array}{c}751.9 \\
43, \\
1.419\end{array}$ \\
\hline 4 & $\begin{array}{l}503- \\
30-0\end{array}$ & Trimethylene oxide & $\begin{array}{l}\mathrm{C}_{3} \mathrm{H}_{6} \\
\mathrm{O}\end{array}$ & $\begin{array}{c}\text { Oxy.Ri } \\
\text { ng }\end{array}$ & $\begin{array}{c}319.9 \\
86, \\
1.485\end{array}$ \\
\hline 3 & $\begin{array}{c}2408 \\
-37- \\
9\end{array}$ & Cyclohexanone, 2,2,6-trimethyl- & $\begin{array}{c}\mathrm{C}_{9} \mathrm{H}_{16} \\
\mathrm{O}\end{array}$ & $\begin{array}{c}\text { Oxy.Ri } \\
\text { ng }\end{array}$ & $\begin{array}{c}1155 . \\
9 \\
1.307\end{array}$ \\
\hline 3 & $\begin{array}{c}3168 \\
-90- \\
9\end{array}$ & Ethanone, 1-(2-methyl-1-cyclopenten-1-yl)- & $\begin{array}{c}\mathrm{C}_{8} \mathrm{H}_{12} \\
\mathrm{O}\end{array}$ & $\begin{array}{c}\text { Oxy.Ri } \\
\text { ng }\end{array}$ & $\begin{array}{c}947.9 \\
23, \\
1.115\end{array}$ \\
\hline 3 & 2312 & Oxetane, 2,2,3-trimethyl- & $\mathrm{C}_{6} \mathrm{H}_{12}$ & Oxy.Ri & 1079. \\
\hline
\end{tabular}




\begin{tabular}{|c|c|c|c|c|c|}
\hline & $0-$ & & $\mathrm{O}$ & ng & 91 \\
\hline & $43-6$ & & & & 1.287 \\
\hline 2 & $\begin{array}{c}3637 \\
-61- \\
4\end{array}$ & Cyclopentanemethanol & $\begin{array}{l}\mathrm{C}_{6} \mathrm{H}_{12} \\
\mathrm{O}\end{array}$ & $\begin{array}{l}\text { Oxy.Ri } \\
\text { ng }\end{array}$ & $\begin{array}{c}1071 . \\
91 \\
1.261\end{array}$ \\
\hline 2 & $\begin{array}{c}1192 \\
-62- \\
7\end{array}$ & Ethanone, 1-(2-furanyl)- & $\begin{array}{c}\mathrm{C}_{6} \mathrm{H}_{6} \\
\mathrm{O}_{2}\end{array}$ & $\begin{array}{l}\text { Oxy.Ri } \\
\text { ng }\end{array}$ & $\begin{array}{l}995.9 \\
18 \\
1.888\end{array}$ \\
\hline 1 & $\begin{array}{c}1355 \\
02- \\
40-8\end{array}$ & 1,3-Benzodioxole, 3a,7a-dihydro-2,2,4-trimethyl- & $\begin{array}{c}\mathrm{C}_{10} \\
\mathrm{H}_{14} \mathrm{O}_{2}\end{array}$ & $\begin{array}{l}\text { Oxy.Ri } \\
\text { ng }\end{array}$ & $\begin{array}{l}947.9 \\
23 \\
1.109\end{array}$ \\
\hline 1 & $\begin{array}{c}0- \\
00-0\end{array}$ & $\begin{array}{l}\text { 1b,5,5,6a-Tetramethyl-octahydro-1-oxa- } \\
\text { cyclopropa[a]inden-6-one }\end{array}$ & $\begin{array}{c}\mathrm{C}_{13} \\
\mathrm{H}_{20} \mathrm{O}_{2}\end{array}$ & $\begin{array}{l}\text { Oxy.Ri } \\
\text { ng }\end{array}$ & $\begin{array}{c}1935 . \\
82 \\
1.478\end{array}$ \\
\hline 1 & $\begin{array}{c}6790 \\
-37- \\
0\end{array}$ & 2-Methyl-3-vinyl-oxirane & $\begin{array}{l}\mathrm{C}_{5} \mathrm{H}_{8} \\
\mathrm{O}\end{array}$ & $\begin{array}{l}\text { Oxy.Ri } \\
\text { ng }\end{array}$ & $\begin{array}{l}583.9 \\
6 \\
1.307\end{array}$ \\
\hline 1 & $\begin{array}{c}1403 \\
5- \\
34-8\end{array}$ & 2,6-Bis(1,1-dimethylethyl)-4-(1-oxopropyl)phenol & $\begin{array}{c}\mathrm{C}_{17} \\
\mathrm{H}_{26} \mathrm{O}_{2}\end{array}$ & $\begin{array}{l}\text { Oxy.Ri } \\
\text { ng }\end{array}$ & $\begin{array}{l}2063 . \\
81 \\
1.465\end{array}$ \\
\hline 1 & $\begin{array}{l}100- \\
73-2\end{array}$ & 2H-Pyran-2-carboxaldehyde, 3,4-dihydro- & $\begin{array}{c}\mathrm{C}_{6} \mathrm{H}_{8} \\
\mathrm{O}_{2}\end{array}$ & $\begin{array}{l}\text { Oxy.Ri } \\
\text { ng }\end{array}$ & $\begin{array}{c}1327 . \\
89 \\
3.742\end{array}$ \\
\hline 1 & $\begin{array}{l}121- \\
00-6\end{array}$ & 3-tert-Butyl-4-hydroxyanisole & $\begin{array}{c}\mathrm{C}_{11} \\
\mathrm{H}_{16} \mathrm{O}_{2}\end{array}$ & $\begin{array}{l}\text { Oxy.Ri } \\
\text { ng }\end{array}$ & $\begin{array}{l}1799 . \\
84 \\
2.251\end{array}$ \\
\hline 1 & $\begin{array}{c}96- \\
08-2\end{array}$ & $\begin{array}{l}\text { 7-Oxabicyclo[4.1.0]heptane, 1-methyl-4-(2- } \\
\text { methyloxiranyl)- }\end{array}$ & $\begin{array}{c}\mathrm{C}_{10} \\
\mathrm{H}_{16} \mathrm{O}_{2}\end{array}$ & $\begin{array}{l}\text { Oxy.Ri } \\
\text { ng }\end{array}$ & $\begin{array}{c}1663 . \\
85 \\
1.254\end{array}$ \\
\hline 1 & $\begin{array}{c}1264 \\
34- \\
25-1\end{array}$ & $\begin{array}{l}\text { Cyclopropanemethanol, } \$, \ddagger \text {-dimethyl-2- } \\
\text { methylene- }\end{array}$ & $\begin{array}{l}\mathrm{C}_{7} \mathrm{H}_{12} \\
\mathrm{O}\end{array}$ & $\begin{array}{l}\text { Oxy.Ri } \\
\text { ng }\end{array}$ & $\begin{array}{c}1075 . \\
91 \\
1.254\end{array}$ \\
\hline 1 & $\begin{array}{c}1467 \\
-36- \\
3\end{array}$ & Ethanone, 1-(2,3,4-trimethylphenyl)- & $\begin{array}{c}\mathrm{C}_{11} \\
\mathrm{H}_{14} \mathrm{O}\end{array}$ & $\begin{array}{l}\text { Oxy.Ri } \\
\text { ng }\end{array}$ & $\begin{array}{c}1563 . \\
86 \\
2.092\end{array}$ \\
\hline 1 & $\begin{array}{c}5633 \\
5- \\
71-8\end{array}$ & Ethanone, 1-(3-ethylcyclobutyl)- & $\begin{array}{l}\mathrm{C}_{8} \mathrm{H}_{14} \\
\mathrm{O}\end{array}$ & $\begin{array}{l}\text { Oxy.Ri } \\
\text { ng }\end{array}$ & $\begin{array}{c}1023 . \\
92 \\
1.129\end{array}$ \\
\hline 1 & $\begin{array}{c}5280 \\
6- \\
35-6\end{array}$ & $\begin{array}{c}\text { Methanone, (4-methoxyphenyl)(6-methyl-1,3- } \\
\text { benzodioxol-5-yl)- }\end{array}$ & $\begin{array}{c}\mathrm{C}_{16} \\
\mathrm{H}_{14} \mathrm{O}_{4}\end{array}$ & $\begin{array}{l}\text { Oxy.Ri } \\
\text { ng }\end{array}$ & $\begin{array}{l}2683 . \\
75 \\
1.742\end{array}$ \\
\hline 1 & $\begin{array}{c}5076 \\
-20- \\
0\end{array}$ & Oxirane, tetramethyl- & $\begin{array}{l}\mathrm{C}_{6} \mathrm{H}_{12} \\
\mathrm{O}\end{array}$ & $\begin{array}{l}\text { Oxy.Ri } \\
\text { ng }\end{array}$ & $\begin{array}{l}699.9 \\
48 \\
1.122\end{array}$ \\
\hline 1 & $\begin{array}{c}5076 \\
-19- \\
7\end{array}$ & Oxirane, trimethyl- & $\begin{array}{l}\mathrm{C}_{5} \mathrm{H}_{10} \\
\quad \mathrm{O}\end{array}$ & $\begin{array}{l}\text { Oxy.Ri } \\
\text { ng }\end{array}$ & $\begin{array}{l}595.9 \\
58 \\
1.162\end{array}$ \\
\hline 9 & $\begin{array}{c}1191 \\
-96- \\
4\end{array}$ & Cyclopropane, ethyl- & $\mathrm{C}_{5} \mathrm{H}_{10}$ & $\begin{array}{l}\text { Satura } \\
\text { ted } \\
\text { Ring }\end{array}$ & $\begin{array}{c}315.9 \\
86, \\
1.063\end{array}$ \\
\hline 3 & $\begin{array}{l}110- \\
82-7\end{array}$ & Cyclohexane & $\mathrm{C}_{6} \mathrm{H}_{12}$ & $\begin{array}{l}\text { Satura } \\
\text { ted }\end{array}$ & $\begin{array}{c}611.9 \\
57\end{array}$ \\
\hline
\end{tabular}




\begin{tabular}{|c|c|c|c|c|c|}
\hline & & & & Ring & 1.036 \\
\hline 2 & $\begin{array}{l}\text { 108- } \\
87-2\end{array}$ & Cyclohexane, methyl- & $\mathrm{C}_{7} \mathrm{H}_{14}$ & $\begin{array}{c}\text { Satura } \\
\text { ted } \\
\text { Ring }\end{array}$ & $\begin{array}{c}719.9 \\
46, \\
1.023\end{array}$ \\
\hline 2 & $\begin{array}{c}3875 \\
-51- \\
2\end{array}$ & Cyclopentane, (1-methylethyl)- & $\mathrm{C}_{8} \mathrm{H}_{16}$ & $\begin{array}{c}\text { Satura } \\
\text { ted } \\
\text { Ring }\end{array}$ & $\begin{array}{c}1103 . \\
91, \\
1.082\end{array}$ \\
\hline 2 & $\begin{array}{c}96- \\
37-7\end{array}$ & Cyclopentane, methyl- & $\mathrm{C}_{6} \mathrm{H}_{12}$ & $\begin{array}{c}\text { Satura } \\
\text { ted } \\
\text { Ring }\end{array}$ & $\begin{array}{c}547.9 \\
63, \\
1.030\end{array}$ \\
\hline 2 & $\begin{array}{c}1978 \\
1- \\
68-1\end{array}$ & Cyclopropane, 1-ethyl-2-methyl-, cis- & $\mathrm{C}_{6} \mathrm{H}_{12}$ & $\begin{array}{c}\text { Satura } \\
\text { ted } \\
\text { Ring }\end{array}$ & $\begin{array}{c}475.9 \\
7, \\
1.036\end{array}$ \\
\hline 2 & $\begin{array}{c}1630 \\
-94- \\
0\end{array}$ & Cyclopropane, 1,1-dimethyl- & $\mathrm{C}_{5} \mathrm{H}_{10}$ & $\begin{array}{c}\text { Satura } \\
\text { ted } \\
\text { Ring }\end{array}$ & $\begin{array}{c}339.9 \\
84, \\
1.049\end{array}$ \\
\hline 2 & $\begin{array}{c}1863 \\
1- \\
83-9\end{array}$ & Cyclopropane, ethylidene- & $\mathrm{C}_{5} \mathrm{H}_{8}$ & $\begin{array}{c}\text { Satura } \\
\text { ted } \\
\text { Ring }\end{array}$ & $\begin{array}{c}391.9 \\
79, \\
1.122\end{array}$ \\
\hline 1 & $\begin{array}{c}6976 \\
-28- \\
9\end{array}$ & 1-Methylpentyl cyclopropane & $\mathrm{C}_{9} \mathrm{H}_{18}$ & $\begin{array}{c}\text { Satura } \\
\text { ted } \\
\text { Ring }\end{array}$ & $\begin{array}{c}951.9 \\
23, \\
0.990\end{array}$ \\
\hline 1 & $\begin{array}{l}591- \\
21-9\end{array}$ & 1,3-Dimethylcyclohexane,c\&t & $\mathrm{C}_{8} \mathrm{H}_{16}$ & $\begin{array}{c}\text { Satura } \\
\text { ted } \\
\text { Ring }\end{array}$ & $\begin{array}{c}811.9 \\
37, \\
1.016\end{array}$ \\
\hline 1 & $\begin{array}{l}185- \\
94-4\end{array}$ & Bicyclo[2.1.0]pentane & $\mathrm{C}_{5} \mathrm{H}_{8}$ & $\begin{array}{c}\text { Satura } \\
\text { ted } \\
\text { Ring }\end{array}$ & $\begin{array}{c}375.9 \\
8, \\
1.122\end{array}$ \\
\hline 1 & $\begin{array}{c}5790 \\
5- \\
86-9\end{array}$ & Cyclobutane, 1,1,2,3,3-pentamethyl- & $\mathrm{C}_{9} \mathrm{H}_{18}$ & $\begin{array}{c}\text { Satura } \\
\text { ted } \\
\text { Ring }\end{array}$ & $\begin{array}{c}1063 . \\
91 \\
1.340\end{array}$ \\
\hline 1 & $\begin{array}{c}4806 \\
-61- \\
5\end{array}$ & Cyclobutane, ethyl- & $\mathrm{C}_{6} \mathrm{H}_{12}$ & $\begin{array}{c}\text { Satura } \\
\text { ted } \\
\text { Ring }\end{array}$ & $\begin{array}{c}547.9 \\
63, \\
1.030\end{array}$ \\
\hline 1 & $\begin{array}{l}598- \\
61-8\end{array}$ & Cyclobutane, methyl- & $\mathrm{C}_{5} \mathrm{H}_{10}$ & $\begin{array}{c}\text { Satura } \\
\text { ted } \\
\text { Ring }\end{array}$ & $\begin{array}{c}667.9 \\
51, \\
1.346\end{array}$ \\
\hline 1 & $\begin{array}{c}4292 \\
-75- \\
5\end{array}$ & Cyclohexane, hexyl- & $\begin{array}{l}\mathrm{C}_{12} \\
\mathrm{H}_{24}\end{array}$ & $\begin{array}{c}\text { Satura } \\
\text { ted } \\
\text { Ring }\end{array}$ & $\begin{array}{c}2107 . \\
81, \\
1.247\end{array}$ \\
\hline 1 & $\begin{array}{c}5410 \\
5- \\
66-7\end{array}$ & Cyclohexane, undecyl- & $\begin{array}{l}\mathrm{C}_{17} \\
\mathrm{H}_{34}\end{array}$ & $\begin{array}{c}\text { Satura } \\
\text { ted } \\
\text { Ring }\end{array}$ & $\begin{array}{c}2279 . \\
79 \\
1.307\end{array}$ \\
\hline 1 & $\begin{array}{l}287- \\
92-3\end{array}$ & Cyclopentane & $\mathrm{C}_{5} \mathrm{H}_{10}$ & $\begin{array}{c}\text { Satura } \\
\text { ted } \\
\text { Ring }\end{array}$ & $\begin{array}{c}311.9 \\
87, \\
1.049\end{array}$ \\
\hline 1 & $\begin{array}{c}7466 \\
3- \\
86-8\end{array}$ & Cyclopropane, 1-ethyl-2-heptyl- & $\begin{array}{l}\mathrm{C}_{12} \\
\mathrm{H}_{24}\end{array}$ & $\begin{array}{c}\text { Satura } \\
\text { ted } \\
\text { Ring }\end{array}$ & $\begin{array}{c}1643 . \\
85, \\
1.142\end{array}$ \\
\hline 1 & $\begin{array}{l}930- \\
18-7\end{array}$ & Cyclopropane, 1,2-dimethyl-, cis- & $\mathrm{C}_{5} \mathrm{H}_{10}$ & $\begin{array}{c}\text { Satura } \\
\text { ted } \\
\text { Ring }\end{array}$ & $\begin{array}{c}311.9 \\
87, \\
1.056\end{array}$ \\
\hline
\end{tabular}




\begin{tabular}{|c|c|c|c|c|c|}
\hline 1 & $\begin{array}{l}118- \\
65-0\end{array}$ & 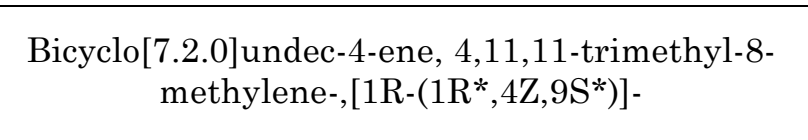 & $\begin{array}{l}\mathrm{C}_{15} \\
\mathrm{H}_{24}\end{array}$ & $\begin{array}{c}\text { Sesquit } \\
\text { erpene }\end{array}$ & $\begin{array}{c}1775 . \\
84, \\
1.366\end{array}$ \\
\hline 1 & $\begin{array}{c}0- \\
00-0\end{array}$ & Di-epi-ł-cedrene & $\begin{array}{l}\mathrm{C}_{15} \\
\mathrm{H}_{24}\end{array}$ & $\begin{array}{l}\text { Sesquit } \\
\text { erpene }\end{array}$ & $\begin{array}{c}1771 . \\
84, \\
1.360\end{array}$ \\
\hline 1 & $\begin{array}{l}473- \\
13-2\end{array}$ & $\begin{array}{l}\text { Naphthalene, } 1,2,3,4,4 \mathrm{a}, 5,6,8 \mathrm{a} \text {-octahydro-4a,8- } \\
\text { dimethyl-2-(1-methylethenyl)-, [2R-(2\$,4a } \neq, 8 \mathrm{a} \cdot)] \text { - }\end{array}$ & $\begin{array}{l}\mathrm{C}_{15} \\
\mathrm{H}_{24}\end{array}$ & $\begin{array}{l}\text { Sesquit } \\
\text { erpene }\end{array}$ & $\begin{array}{c}1839 . \\
83 \\
1.432\end{array}$ \\
\hline 22 & $\begin{array}{c}75- \\
18-3\end{array}$ & Dimethyl sulfide & $\begin{array}{l}\mathrm{C}_{2} \mathrm{H}_{6} \\
\mathrm{~S}\end{array}$ & Sulfur & $\begin{array}{l}359.9 \\
82 \\
1.280\end{array}$ \\
\hline 2 & $\begin{array}{l}3550 \\
8- \\
06-6\end{array}$ & 1-Mercapto-2-propanol, acetate & $\begin{array}{l}\mathrm{C}_{5} \mathrm{H}_{10} \\
\mathrm{O}_{2} \mathrm{~S}\end{array}$ & Sulfur & $\begin{array}{c}1279 . \\
89 \\
1.511\end{array}$ \\
\hline 2 & $\begin{array}{l}2995 \\
6- \\
99-8\end{array}$ & Disulfide, bis(1,1,3,3-tetramethylbutyl) & $\begin{array}{c}\mathrm{C}_{16} \\
\mathrm{H}_{34} \mathrm{~S}_{2}\end{array}$ & Sulfur & $\begin{array}{l}1243 . \\
89 \\
1.940\end{array}$ \\
\hline 1 & $\begin{array}{l}637- \\
89-8\end{array}$ & 4-Mercaptophenol & $\begin{array}{l}\mathrm{C}_{6} \mathrm{H}_{6} \\
\mathrm{OS}\end{array}$ & Sulfur & $\begin{array}{c}1075 . \\
91 \\
1.247\end{array}$ \\
\hline 1 & $\begin{array}{c}0- \\
00-0\end{array}$ & 5-Oxohexanethioic acid, S-t-butyl ester & $\begin{array}{l}\mathrm{C}_{10} \\
\mathrm{H}_{18} \mathrm{O}_{2} \\
\quad \mathrm{~S}\end{array}$ & Sulfur & $\begin{array}{c}1243 . \\
89 \\
1.940\end{array}$ \\
\hline 1 & $\begin{array}{c}75- \\
15-0\end{array}$ & Carbon disulfide & $\mathrm{CS}_{2}$ & Sulfur & $\begin{array}{c}387.9 \\
79 \\
1.208\end{array}$ \\
\hline 1 & $\begin{array}{c}3326 \\
6- \\
06-7\end{array}$ & Ketone, methyl 2-methyl-1,3-oxothiolan-2-yl & $\begin{array}{c}\mathrm{C}_{6} \mathrm{H}_{10} \\
\mathrm{O}_{2} \mathrm{~S}\end{array}$ & Sulfur & $\begin{array}{c}715.9 \\
46, \\
1.181\end{array}$ \\
\hline 1 & $\begin{array}{l}124- \\
63-0\end{array}$ & Methanesulfonyl chloride & $\begin{array}{c}\mathrm{CH}_{3} \\
\mathrm{ClO}_{2} \\
\mathrm{~S}\end{array}$ & Sulfur & $\begin{array}{c}623.9 \\
56, \\
2.086\end{array}$ \\
\hline 1 & $\begin{array}{c}0- \\
00-0\end{array}$ & Sulfurous acid, hexyl tetradecyl ester & $\begin{array}{c}\mathrm{C}_{20} \\
\mathrm{H}_{42} \mathrm{O}_{3} \\
\quad \mathrm{~S}\end{array}$ & Sulfur & $\begin{array}{c}2235 . \\
79 \\
1.175\end{array}$ \\
\hline 1 & $\begin{array}{c}6317 \\
-18- \\
6\end{array}$ & Thiocyanic acid, methylene ester & $\begin{array}{l}\mathrm{C}_{3} \mathrm{H}_{2} \\
\mathrm{~N}_{2} \mathrm{~S}_{2}\end{array}$ & Sulfur & $\begin{array}{c}831.9 \\
35, \\
1.221\end{array}$ \\
\hline 2 & $\begin{array}{c}6751 \\
7- \\
14-0\end{array}$ & Neoisolongifolene, 8,9-dehydro- & $\begin{array}{l}\mathrm{C}_{15} \\
\mathrm{H}_{22}\end{array}$ & $\begin{array}{l}\text { Unsatu } \\
\text { rated } \\
\text { Ring }\end{array}$ & $\begin{array}{c}1959 . \\
82, \\
1.630\end{array}$ \\
\hline 1 & $\begin{array}{l}629- \\
20-9\end{array}$ & 1,3,5,7-Cyclooctatetraene & $\mathrm{C}_{8} \mathrm{H}_{8}$ & $\begin{array}{l}\text { Unsatu } \\
\text { rated } \\
\text { Ring }\end{array}$ & $\begin{array}{c}979.9 \\
2, \\
1.307\end{array}$ \\
\hline 1 & $\begin{array}{c}5187 \\
4- \\
62-5\end{array}$ & 4-t-Pentylcyclohexene & $\begin{array}{l}\mathrm{C}_{11} \\
\mathrm{H}_{20}\end{array}$ & $\begin{array}{l}\text { Unsatu } \\
\text { rated } \\
\text { Ring }\end{array}$ & $\begin{array}{c}1075 . \\
91 \\
1.228\end{array}$ \\
\hline 1 & $\begin{array}{l}275- \\
51-4\end{array}$ & Azulene & $\mathrm{C}_{10} \mathrm{H}_{8}$ & $\begin{array}{l}\text { Unsatu } \\
\text { rated } \\
\text { Ring }\end{array}$ & $\begin{array}{c}1403 . \\
88, \\
2.158\end{array}$ \\
\hline 1 & 5293 & Cyclohexene, 2-ethenyl-1,3,3-trimethyl- & $\mathrm{C}_{11}$ & Unsatu & 1251. \\
\hline
\end{tabular}




\begin{tabular}{|c|c|c|c|c|c|}
\hline & $-90-$ & & $\mathrm{H}_{18}$ & $\begin{array}{l}\text { rated } \\
\text { Ring }\end{array}$ & 89 \\
\hline & 3 & & & Ring & 1.393 \\
\hline 1 & $\begin{array}{c}0- \\
00-0\end{array}$ & Cycloisolongifolene, 9,10-dehydro- & $\begin{array}{l}\mathrm{C}_{15} \\
\mathrm{H}_{22}\end{array}$ & $\begin{array}{l}\text { Unsatu } \\
\text { rated } \\
\text { Ring }\end{array}$ & $\begin{array}{c}1959 . \\
82, \\
1.624\end{array}$ \\
\hline 1 & $\begin{array}{c}2149 \\
733\end{array}$ & Deltacyclene & $\mathrm{C}_{9} \mathrm{H}_{10}$ & $\begin{array}{l}\text { Unsatu } \\
\text { rated } \\
\text { Ring }\end{array}$ & $\begin{array}{c}1163 . \\
9 \\
1.379\end{array}$ \\
\hline 1 & $\begin{array}{c}91- \\
20-3\end{array}$ & Naphthalene & $\mathrm{C}_{10} \mathrm{H}_{8}$ & $\begin{array}{l}\text { Unsatu } \\
\text { rated } \\
\text { Ring }\end{array}$ & $\begin{array}{c}1403 . \\
88 \\
2.158\end{array}$ \\
\hline 1 & $\begin{array}{c}2156 \\
4- \\
79-4 \\
\end{array}$ & Naphthalene, 1,2-dihydro-2-methyl- & $\begin{array}{l}\mathrm{C}_{11} \\
\mathrm{H}_{12}\end{array}$ & $\begin{array}{l}\text { Unsatu } \\
\text { rated } \\
\text { Ring }\end{array}$ & $\begin{array}{c}1523 . \\
87 \\
1.874\end{array}$ \\
\hline
\end{tabular}


Table A.A2 ANOVA and column statistics for variables measured by Springtail/Water Spray treatment.

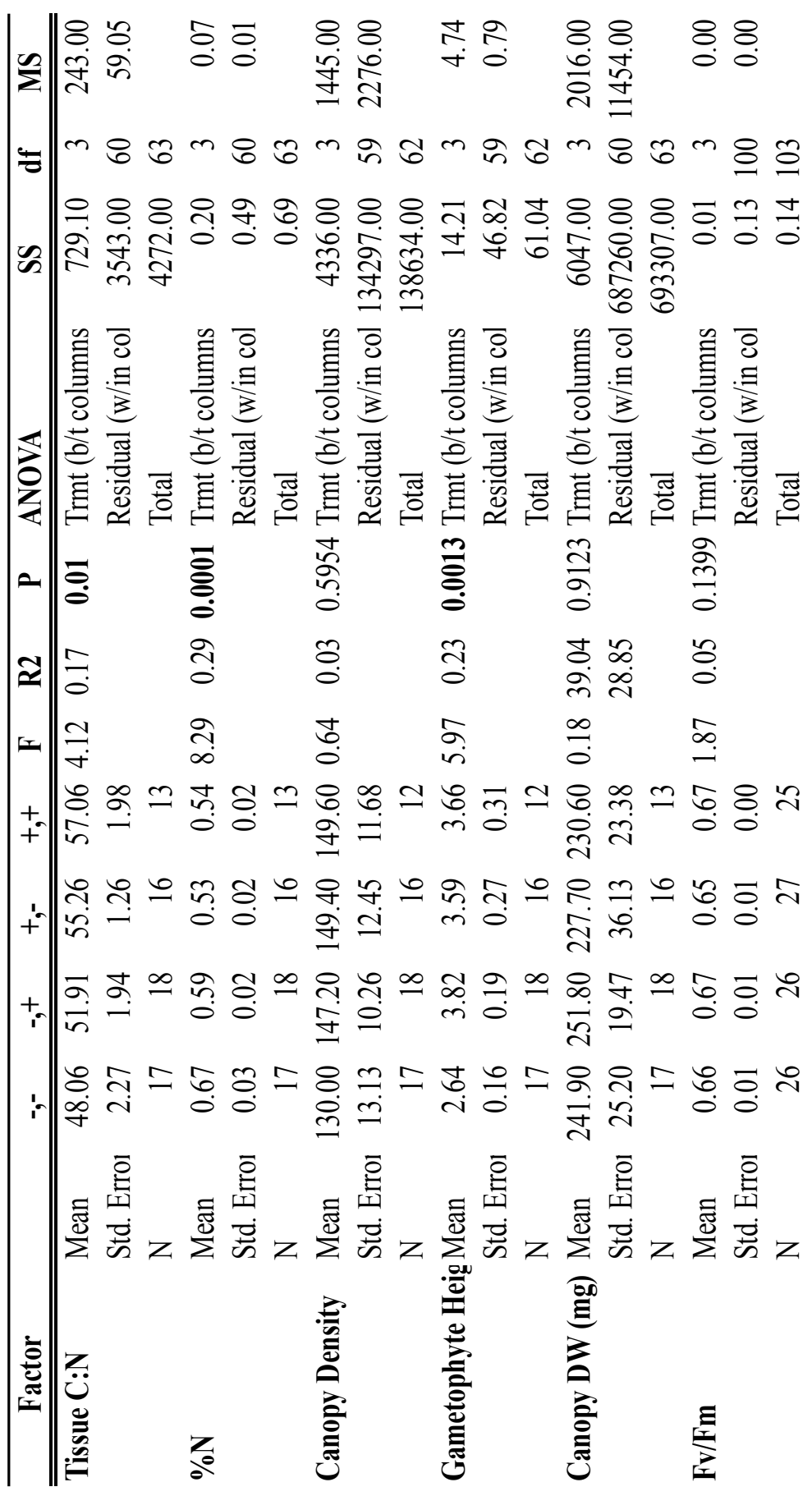


Figure A. A.1 Total number of volatile organic compounds detected from the headspace of matched populations of mosses grown in a common garden greenhouse. C. purpureus females originated from two Portland, OR populations emitted $104.0 \pm 12.1 \mathrm{SE} ; \mathrm{N}=11$, and males of the same populations emitted $31.9 \pm 6.70 \mathrm{SE} ; \mathrm{N}=11 ; \mathrm{P}<0.0001$ compounds as detected by GCxGC analysis. B. argenteum females from two Portland, OR populations emitted $155.7 \pm 71.50 \mathrm{SE} ; \mathrm{N}=3$, and matched males emitted 56.00 $\pm 20.40 \mathrm{SE} ; \mathrm{N}=3 ; \mathrm{P}=0.25$ compounds.

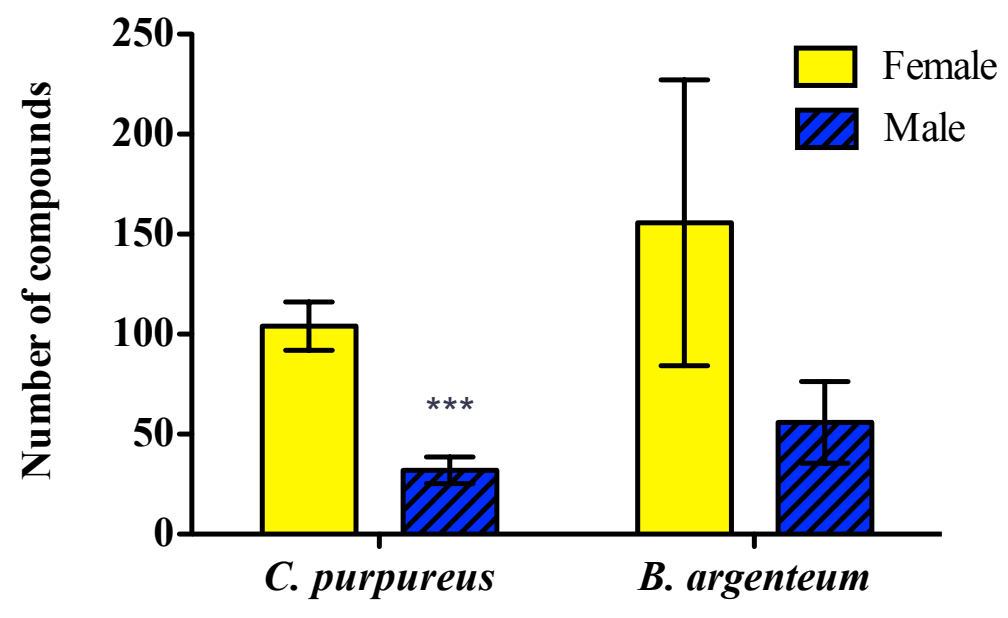


Figure A.A2 Moss Volatile Organic Compounds from C. purpureus individuals by sex and IAUPAC compound class. Symbols represent data mean, bars are \pm SE. Females are represented by circles, $\mathrm{N}=1$; males are represented by triangles, $\mathrm{N}=14$. 2-Way ANOVA reveal significant differences between males and females $(\mathrm{P}=0.003)$ and between compound classes $(\mathrm{P}<0.0001)$. $)$. Y-axis shows peak area under the curve $(\log )$, a relative measure of compound abundance.

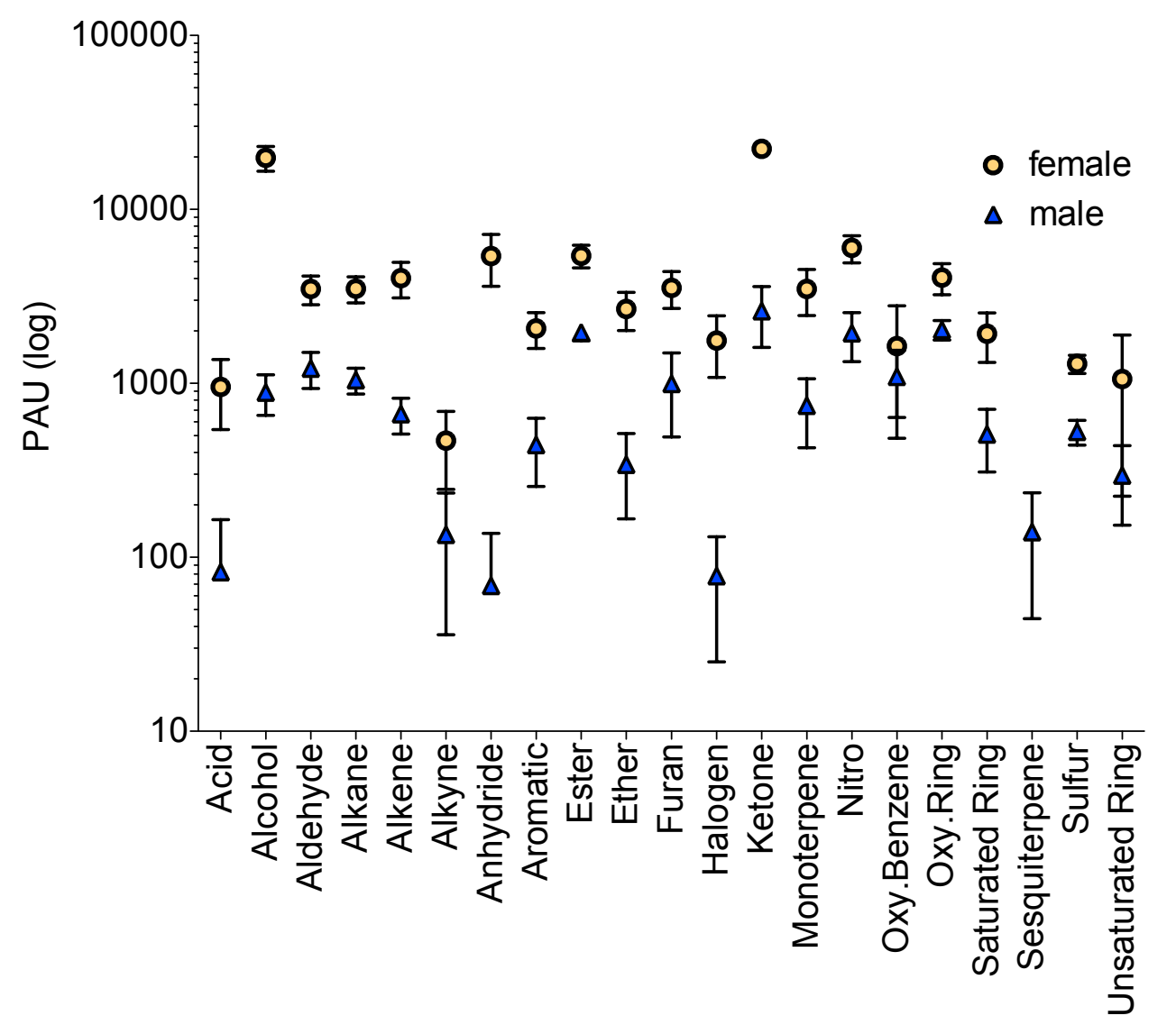


Appendix A.A3 Volatile profiles binned by IAUPAC compound class of five representative male and female C. purpureus mosses. a) five female mosses vary from one another significantly $(\mathrm{F}=3.26, \mathrm{P}=0.02)$ and have greater overall compound PAC than males, b) five male mosses differ from one another significantly $(\mathrm{F}=2.52 ; \mathrm{P}=0.05)$. $\mathrm{Y}$-axis shows peak area under the curve, a relative measure of compound abundance.

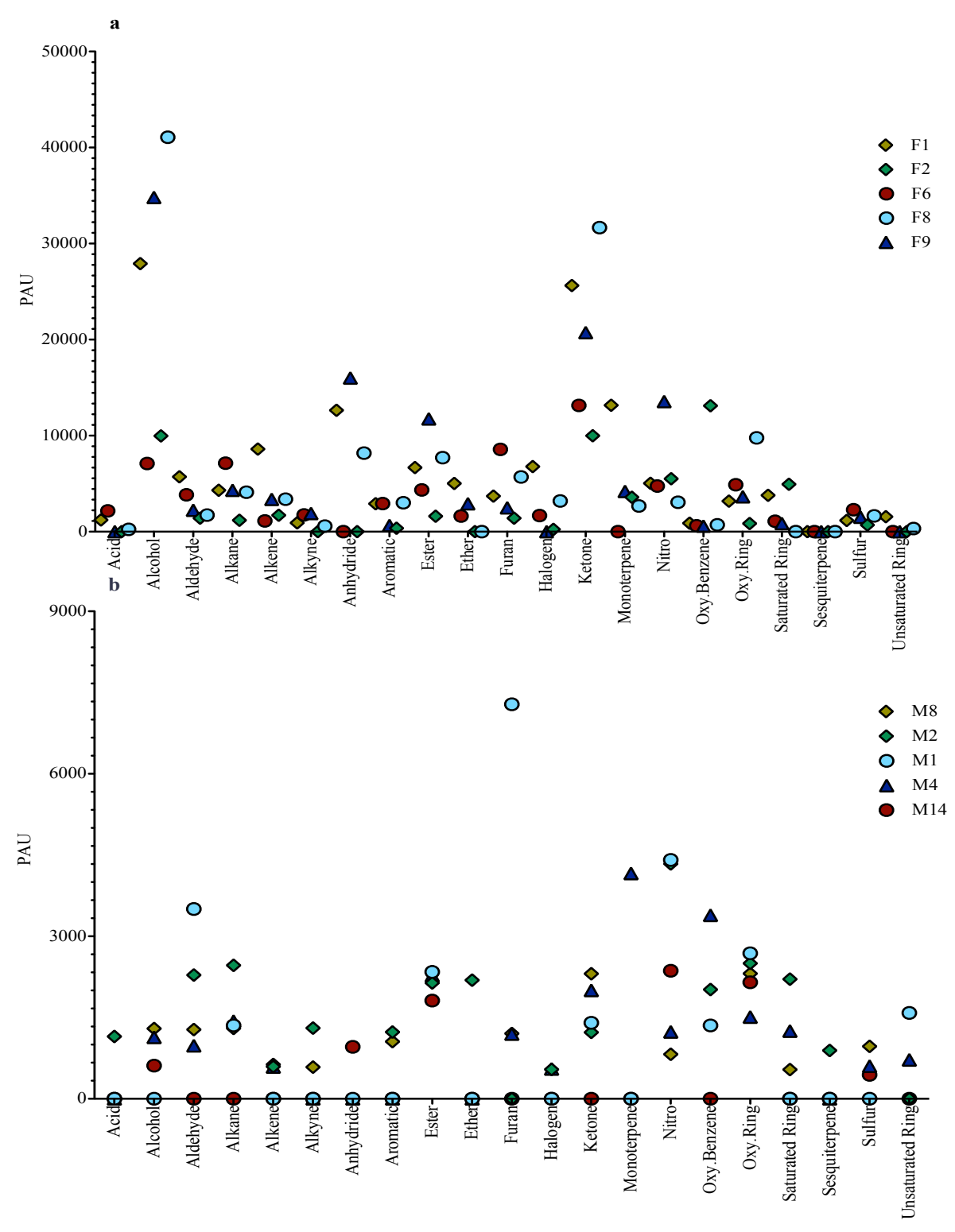

IUPAC Compound Class 
Fig. A.A4 Microcosm canopy measurements. Columns represent data mean by treatment, bars are \pm SE. Data for a-e are averaged values from $5-6 \mathrm{~mm}^{2}$ cores taken from each microcosm. Fv/Fm values are means of averaged data points from each microcosm by treatment a) Tissue $\mathrm{C}: \mathrm{N}$, b) percent tissue $\mathrm{N}$, c) gametophye density (mean number per core per microcosm), d) gametophyte height of three randomly chosen gametophytes per core ( $\mathrm{mm}), \mathrm{e})$ canopy dry weight of moss tissue (mg) f) efficiency of PSII by dark-adapted Fv/Fm measurements. ANOVA and column statistics are in Table A.A1
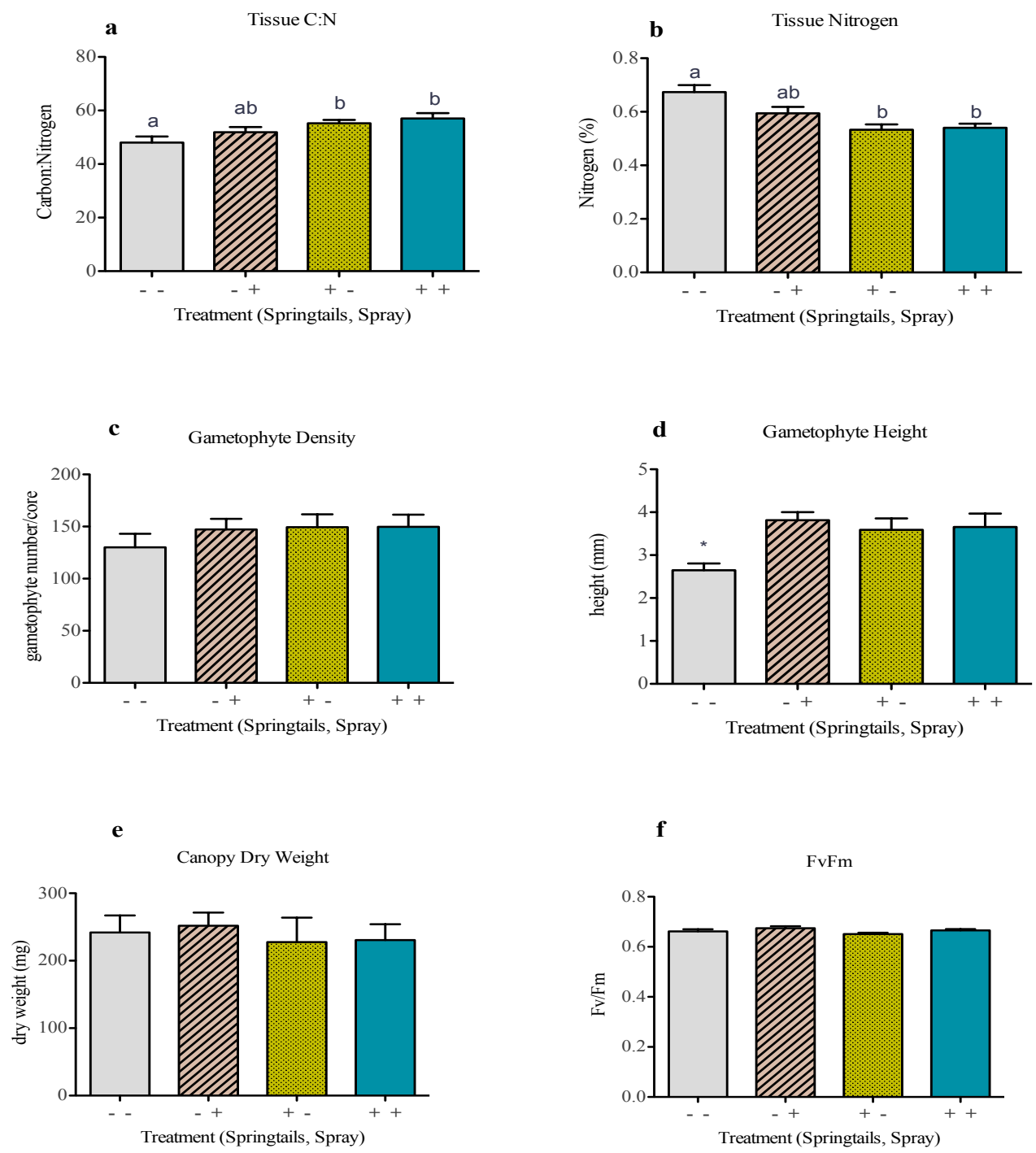
**Data represented in Fig. A.A4 were not included in Chapter 2 as they were not significant in our full mixed-model ANOVA statistical analyses. Data here represent raw numerical differences among and between treatments without tray, growth chamber effect, or that springtail treatment is nested in tray. 


\section{Appendix B}

\section{Testing biotic and abiotic influences on the reproductive biology of the monoicous moss, Physcomitrella patens}

\section{Introduction}

The majority of flowering plants are hermaphrodites, (monoecious)

(Charlesworth, 1985), yet many of these plants form associations with pollinators or use abiotic vectors for pollen transport in effort to avoid selffertilization. Outcrossing thus insures lowered rates of self-fertilization and therefore potentially avoids inbreeding depression (Ashman, 2009, Holsinger, 2000, Lloyd et al., 1996). Little is known about whether monoicous mosses suffer from a similar predicament yet hermaphrodites have the advantage of not having to find a mate for successful sporophyte production (Longton, 2006). Mosses with separate sexes or dioicous mosses ( $60 \%$ of known species) (Wyatt \& Anderson, 1984) are generally presumed to be dispersal limited, and monoicous mosses less limited assuming the moss is selfcompatible.

Sporophyte inbreeding depression as a result of self-fertilization is shown to be quite low in monoicous mosses, yet high in dioicous species (Jesson et al., 2007). These data indicate that the variable haploid-dominant mating systems may have different evolutionary trajectories, allowing the self-fertilizing mosses to purge deleterious alleles, ultimately eliminating 
inbreeding depression. The evolutionary argument against self-fertilization is that in a changing environment, those clones may not survive yet an organism with recombined genes may. Further, a greater heterozyogote deficiency was found in monoicous mosses over dioicous mosses (Eppley et al., 2006) and there is pronounced post-zygotic selection for more heterozygous sporophytes by female dioicous peat mosses (Szövényi et al., 2009), thus indicating that dioicous mosses tend to favor heterozygosity while monoicous mosses do not.

To our knowledge, all moss canopies maintain a diverse microbiota (Bragina et al., 2013, Davey \& Currah, 2006) and thus provide food sources and opportune sheltered microclimates for microarthropods (Andrew et al., 2003b, Gerson, 1969). Dioicous mosses gain greater reproductive success through their association with both microarthropods acting as biotic dispersal vectors in addition abiotic dispersal vectors. Bulk water flow, and the newly documented microarthropod vector, Collembola (Cronberg et al., 2006, Rosenstiel et al., 2012) are the known mechanisms for moss sperm dispersal. What has not been investigated is if monoicous species also yield greater sporophyte production when in association with microarthropods. To determine whether the addition of Collembola (Sinella curviseta, Folsomia candida) or water spray increase fertilization and outcrossing rates in a highly-selfing monoicous moss, I designed laboratory microcosm experiments using the model moss species, Physcomitrella patens. P. patens is a mostly 
laboratory grown moss species that has been used extensively in studies of genetics, evolution, stress tolerance, genomics, development, and biotechnology (Cove, 2005, Prigge \& Bezanilla, 2010, Quatrano et al., 2007, Rensing et al., 2008, Schaefer, 2002), but much less so in an ecological context.

Strains of $P$. patens have been genetically transformed to express green fluorescent protein (GFP), or red fluorescent protein (RFP). The transformed mosses were shown to predictably express the fluorescent proteins and to be capable of outcrossing, evidenced by a hybrid sporophyte (Perroud et al., 2011). I aimed to take advantage of this excellent system to test not only the rate and magnitude of sporophyte production with different sperm dispersal agents in $P$. patens, but also the genetic effects of the various sperm vectors. Microarthropods moving sperm between individual mosses, both monoicous and dioicous could result in increased genetic diversity, potentially more so than with water alone. If true, a moss-microarthropod mutualism would have key characters akin to that of the flowering plant fitness benefits from involvement with pollinators (Loveless \& Hamrick, 1984).

In this study, I test the relative effects of two potential sperm vectors (Collembola and water spray), and the combination of the two on three laboratory strains of $P$. patens in two ways: 1) using each strain alone, and 2) in factorial arrays, intended to allow for potential outcrossing between the three lines. For the single strains alone, I predicted that there would not be 
a significant increase in sporophyte production with the addition of microarthropods on $P$. patens, but instead, a marginal increase in sporophyte production with both microarthropod and water spray treatments. For the factorial out-crossing experiments, I predicted that the addition of microarthropods would increase evidence of outcrossing even against a high background rate of self-fertilization.

\section{Materials and Methods}

Both genetically modified and unmodified Physcomitrella patens (Hedw) strains were used for all experiments. Plant material was obtained from existing laboratory cultures in St. Louis, MO (courtesy of FP Perroud). I used material from strains of wild type P. patens Villersexel (VxWt France) and 'Grandsen 2004' (GdWt) strains, as well as Villersexel individuals transformed to express the RFP (VxRFP) protein and Grandsen strains transformed to express GFP (GdGFP) proteins. All lines were found to maintain stable fluorophore expression. For details on strain transformation and origins see (Perroud et al., 2011).

Cultures were propagated by homogenization of green tissue from each individual P. patens strain (ProScientific, MD) in sterilized tap water (MultiGen 7XL Homogenizer, ProScientific Inc., CT). The homogenized moss solution was transferred via pipette to 12 well-plates with either $B C D$ media designed for the growth of $P$. patens (Cove et al., 2009). Cultures were grown 
in controlled growth chambers (Adaptis A1000, Conviron) at $21^{\circ} \mathrm{C}$ with a 12 hour photoperiod (light levels 50-100uE) for four weeks. A subset of each strain of $P$. patens was examined with fluorescent microscopy to verify the expression of the respective expected flourophores.

\section{Sporophyte production experiment}

After four-five weeks at constant temperature conditions as described above, mosses of VxWt, GdGFP and VxRFP and their surrounding nutrient agar were transferred from BCD media plates to magenta jars (microcosms) with a sterile media of 2:1 sand, peat mixture (Fig. A.B1). Transferred cultures were moved into a gametoecia-inducing growth chamber environment (Cove et al., 2009) of $15^{\circ} \mathrm{C}$ and an eight hour photoperiod $(\sim 100 \mu \mathrm{E})$ with constant relative humidity of $75 \%$ until sporophytes formed in abundance (4-8 weeks). Each microcosm received one of the following treatments: 1) sterilized spring water spray ( $\sim \mathrm{mL}$ per spray), 2) Collembola ( 15-20 individual adult Folsomia candida), 3) neither treatment, or 4) both treatments. Six wild type microcosms were established for each treatment and three microcosms of each transformed line were established for each treatment. In the microcosms without water spray treatments, $1 \mathrm{~mL}$ of water was added to the substrate each time the microcosm were sprayed in order to keep the microcosms equally hydrated, but avoiding abiotic sperm transfer by means of water spray. Spray treatments were repeated once every week until mass 
sporophyte production occurred. Springtails were added again after one month to the appropriate microcosms.

Sixty days after propagation, one $12 \mathrm{~mm}$ core was taken from the center of each moss patch from each microcosm for analysis. Each gametophyte per core, number of sporophytes per core and the heights of three randomly chosen gametophytes were recorded.

Collected data was analyzed using mixed-model ANOVA to assess the relative contributions of variables collected to sporophyte production as well as variances between treatments in variables collected. Statistical analyses were conduced using JMP10 (SAS Institute, 2012).

\section{Methods and Results - Outcrossing experiment}

P. patens lines were propagated as outlined previously. Upon gametophyte maturation at constant temperature, $\mathrm{P}$. patens individual plugs were transferred to either sterile $2 ; 1$ sand soil substrate or sterilized Turface MVP substrate in Magenta jars.

Methods. Two experiments of 48 jars apiece were established. All substrates were equally watered. Jars were established with both pairwise microcosms of: each strain with itself (GdWt-GdWt VxWt-VxWt, GdGFPGdGFP, VxRFP-VxRFP) and with one other strain (GdWt-GdGFP, GdWtVxRFP, GdGFP-VxRFP, VxWt-GdGFP, VxWt-VxRFP), as well as all three strains represented in the same jar. I was unable to attain VxGFP and 
GdRFP lines to complete a full-factorial design. I chose to test only the effects of microarthropods on outcrossing in these experiments and to supply all microcosms with adequate water spray. Half of each outcrossing design had 15-20 F. candida added to each jar two times over the 4-6 week development time in the gametoecia-inducing growth chamber environment.

Results. Upon abundant sporophyte production in all jars, (P. patens is highly fecund and nearly every shoot develops a sporophyte after 1-2 months in the proper environmental conditions) mosses were examined under compound microscopy and fluorescent tissue of the transformed lines was verified with fluorescent microscopy. (Olympus BX60, epifluorescent compound microscope).

A variety of microscopes and filter sets were used in order to optimize viewing as after many attempts, I was not confident that I would be able to accurately determine autofluorescence from fluorophore fluorescence in a hybrid. Moss tissues are highly autofluorescent under excitation.

Presumably the sporophyte tissue is diploid and therefore will be a product of the mother and father moss. If a GFP labeled male for example fertilizes a RFP female then the sporophyte should emit both fluorophores under proper excitation wavelengths. Similarly, if a non-labeled parental Gransden moss were fertilized by a GFP labeled male moss sperm, then the sporophyte should express GFP proteins. Multiple filter sets were tested to attempt to visualize both GFP and mCherry (the RFP flourophore) in the same view. 
With various filter set-ups I was able to distinguish each strain from one another without doubt, but I was not able to obtain proper confidence in my ability to discern if a sporophyte was expressing both fluorophores without a positive control. It was determined that the potential instrumentation cost of establishing the best possible microscope-filter combination, and still unresolved uncertainty in the data collection methods, the project was terminated.

Accurately visualizing tissues with GFP fluorophores only (Olympus BX60 epifluorescent compound microscope) was possible, as the excitation wavelength to excite GFP does not thoroughly excite autofluorescent moss tissue. In a subsequent experiment of GdGFP/GdWt only arrays, strains were grown side-by-side in the previously described methods (Fig. A.B1). Additionally, mosses that were all flooded with sterile tap water to induce reproduction following suggested protocols (Cove et al., 2009) and also treated with water spray and/or Collembola. Over 300 sporophytes of the GdWt strain were randomly assessed for GFP expression in the sporophyte. I found no GdWt sporophytes expressing the GFP protein in the sporophyte as detectable by available instrumentation, although the GdGFP individuals were easily identified by microscopy as expected. Thus, these findings cannot support previous findings revealing a $3 \%$ outcrossing rate in the transformed strains with water flooding alone (Perroud et al., 2011), nor do they support 
the hypothesis that $P$. patens would show evidence of outcrossing under biotic and abiotic manipulations.

\section{Results - Single-strain sporophyte production experiment}

Overall, I found that at the time of collection, the mean number of sporophytes per core varied significantly by strain, with the wild type producing significantly more sporophytes (mean $20.3 \pm 1.51 ; \mathrm{N}=24$ ) than GdGFP or VxRFP. GdGFP produced significantly more sporophytes per core (mean $9.2 \pm 1.51, \mathrm{~N}=12$ ) than RFP (mean $2.3 \pm 0.68$; N=12), (Fig. A.B2, Fig. A.B3a $; \mathrm{F}$ ratio $=39.30 ; \mathrm{P}<0.0001)$. Similarly, the average height of wild type gametophytes was taller than either transformed strain $($ Fig. A.B3b; F ratio $=$ 17.53; $\mathrm{P}<0.0001)$. I also found that there were differential gametophyte densities by strain with GdWt having slightly but significantly lower gametophyte mean per core (Fig. A.B3c; F ratio 6.59; $\mathrm{P}=0.003$ ).

Gametophyte density and height by treatments and strain are depicted in Figure A.B3. The treatment by strain analysis shows no overall significance, but there were strain by treatment differences (For Tukey HSD significance: Table AB.1)

\section{Discussion}

The most interesting finding in this study is that transformed $P$. patens strains are not all equally fecund, but this does not appear to be due to any of 
the treatments applied to the strains. The wild type (VxWT) consistently produced the most sporophytes over either transformed line (Fig. A.B2). It is possible that the transformed strains are simply slower to develop, but all microcosms were initially propagated at the same time with the same amount of tissue. Sporophyte harvest was delayed by an additional two weeks to attempt to wait for all three lines to be seemingly equivalent in sporophyte yield, but the tissues began to look yellow, indicating declining health and cores from all microcosms were extracted.

The wild type strain did not have significant differences in percent sporophyte yield between treatments, but the wild type microcosms without Collembola had almost $80 \%$ of gametophytes bearing sporophytes, but less in microcosms with Collembola added (Fig. A.B3a; 70\% (+,+) and 58\% (+,-)) indicating that perhaps the addition of the Collembola was in fact detrimental to sporophyte yield in the wild type strain, directly contrary to similar tests with dioicous species (Cronberg et al., 2006, Rosenstiel et al., 2012). The lowest sporophyte yield in the wild type strain was with the Collembola/no water treatment, this treatment also yielded the highest mean sporophyte percentage in a transformed line (Fig. A.B3a; GFP, 59\% (+,-)). The same treatment also shows the lowest gametophyte density in the GFP strain (Fig. A.B3c). Interestingly, both transformed lines with all treatments except for the aforementioned GFP (+.-) show higher gametophyte densities, 
indicating that their efforts could be going towards primary production (Waite \& Sack, 2010)

These data highlight a potentially interesting avenue for future studies. P. patens is a laboratory moss, thus is highly adapted to life in growth chambers and has essentially been bred to be an extremely efficient selffertilizer, thus it is not at all surprising that adding a treatment to these already uber-fecund strains has no positive effect. On the other hand, the transformed strains do not appear to have identical vigor to the Wt strains, indicating that perhaps there is a reproductive trade-off for expressing fluorescent proteins. Further tests on the physiology and reproductive biology of the transformed strains compared to the wild type strains may be pertinent, particularly if the tranformants are expected to perform as the wild types do in laboratory experiments.

The outcrossing experiment did not yield results. I postulate this could be due to one of three reasons: 1) I lacked the instrumentation to adequately assess and observe hybrid sporophytes via fluorescent microscopy, 2) there were no hybrid sporophytes produced, or 3) the sporophyte capsule itself is not fully diploid tissue and/or the proteins were not expressed in the sporophyte capsule stage. Unfortunately, I cannot determine which of these the actual reasons are. If there is a very strong selection pressure to selffertilize it is possible that no hybrids were produced due to pre-zygotic barriers (Szovenyi et al., 2009). Further, I used conditions specifically 
designed for highly fecund $P$. patens, if the strains were acclimated to a less defined environment, or I used a more common abundant monoicous species, perhaps there would be more room for diverse vectors for transport.

Another possible explanation for the lack of increased sporophyte production with biotic vectors is that monoicous mosses may not have evolved the relationship to microarthropods that dioicous mosses have.

Hermaphroditic mosses have been found to be relatively free of inbreeding depression, thus do not suffer from self-fertilization the way that dioicous mosses do (Eppley et al., 2007, Jesson et al., 2007, Szovenyi et al., 2009). If a monoicous moss does not suffer from selfing and easily can self due to proximity alone, there may not be any pressure to evolve a relationship with microarthropods such as investing in volatile cues (Marino et al., 2009, Rosenstiel et al., 2012). Another possibility is that in monoicous species, Collembola are not the ideal invertebrate to use in experimental tests. Possibly mites or another common moss-dwelling microarthropod would be a better choice as they cover much less distance in the canopies, often travelling on the same gametophyte and not among and in between gametophyte patches as Collembola do (pers obs).

Moss mating systems are a grossly understudied aspect of bryophyte biology, particularly when compared to their younger flowering relatives, yet intriguing new research on the evolution of dioecy in land plants indicate that mosses are consistently evolving dioicy (McDaniel et al., 2013a), unlike 
flowering plants who tend to be hermaphroditic or of a mixed sexual system (Geber et al., 1999). Bryophytes have been evolving over hundreds of millions of years and our understanding of the mechanisms and interactions effecting moss reproduction is in its infancy. This work only confirms that there are previously untested differences between the mating systems of mosses as well as species-specific differences. This work also highlights that there are potential ramifications for genetic modification that could go unnoticed without controlled comparisons and it is important to qualify when and where it is acceptable and rational to use modified organisms to ask ecological and biological questions. Although this study did not uncover the mystery of monoicous moss relationships to microarthropods, it presents a platform by which to launch further studies and highlights the magnitude of what we don't know about what lies just below our feet. 


\section{Tables and Figures}

Table A.B1 Tukey's HSD post hoc analysis results for mixed model ANOVA on the effects of strain by treatment (+ or - invertebrates) on total sporophyte production.

\begin{tabular}{|c|c|c|c|c|c|}
\hline Level & & & & & $\begin{array}{l}\text { Least Square } \\
\text { Mean }\end{array}$ \\
\hline -+, WildType & $\overline{\mathrm{A}}$ & & & & 22.33 \\
\hline --, WildType & $\mathrm{A}$ & & & & 22 \\
\hline +-, WildType & $\mathrm{A}$ & B & & & 19 \\
\hline ++, WildType & $\mathrm{A}$ & B & $\mathrm{C}$ & & 18 \\
\hline,+- GFP & $\mathrm{A}$ & B & $\mathrm{C}$ & $\mathrm{D}$ & 11 \\
\hline,-- GFP & $\mathrm{A}$ & B & $\mathrm{C}$ & $\mathrm{D}$ & 10.67 \\
\hline,-+ GFP & $\mathrm{A}$ & B & $\mathrm{C}$ & $\mathrm{D}$ & 9.67 \\
\hline,++ GFP & & B & $\mathrm{C}$ & $\mathrm{D}$ & 5.33 \\
\hline +-, RFP & & B & $\mathrm{C}$ & $\mathrm{D}$ & 3.67 \\
\hline,++ RFP & & & $\mathrm{C}$ & $\mathrm{D}$ & 2.67 \\
\hline,$-+ \mathrm{RFP}$ & & & & $\mathrm{D}$ & 2 \\
\hline,-- RFP & & & & $\mathrm{D}$ & 1 \\
\hline
\end{tabular}


Figure A.B1 Growth environments of P. patens a) P. patens on BCD agar after four weeks, b) a pairwise $P$. patens microcosm and c) three-way experimental microcosms.

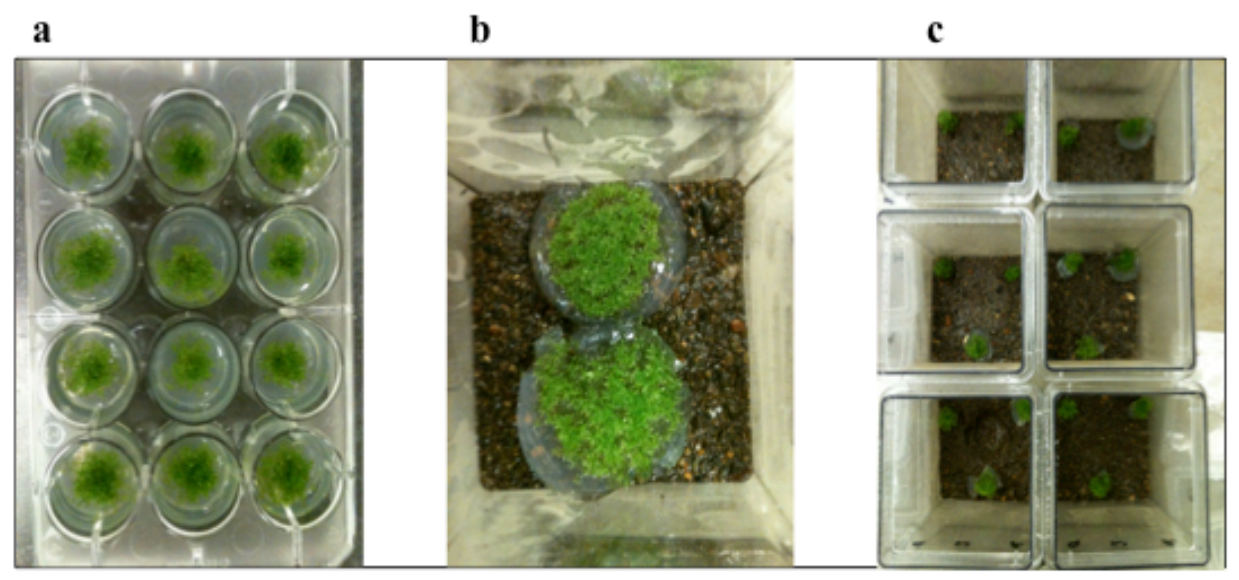


Figure A.B2 Total number of sporophytes produced in microcosm arrays. Columns represent mean sporophyte number, bars are $\pm \mathrm{SE}$.

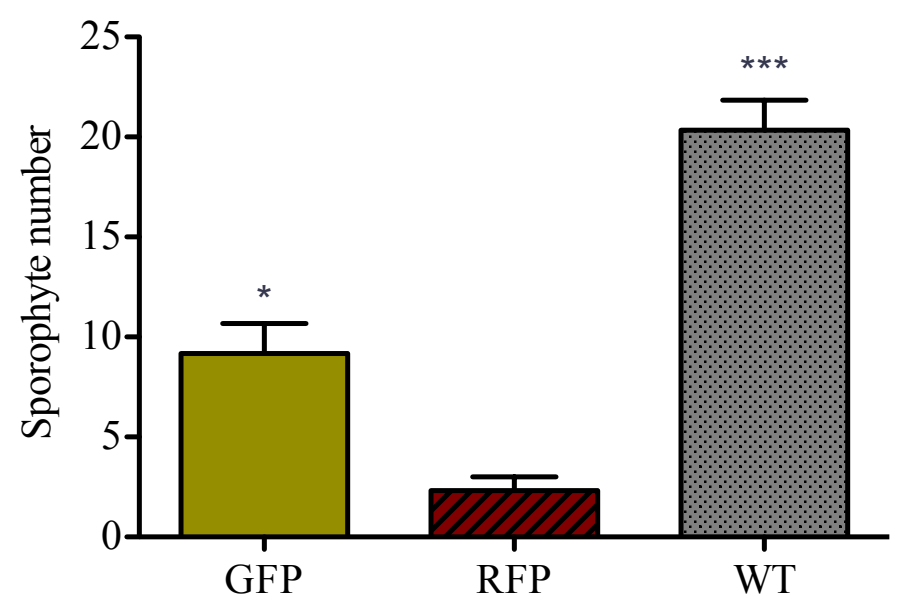


Figure A.B3 Three P. patens strains responses to four fertilization treatments. Symbols are data mean, error bars represent \pm SE. Circles are wild type strains, triangles represent RFP, and squares represent GFP strains. a) Percent of gametophytes counted bearing sporophytes by treatment, b) average height of three random gametophytes of each strain by treatment, and c) gametophyte density of each strain by treatment.
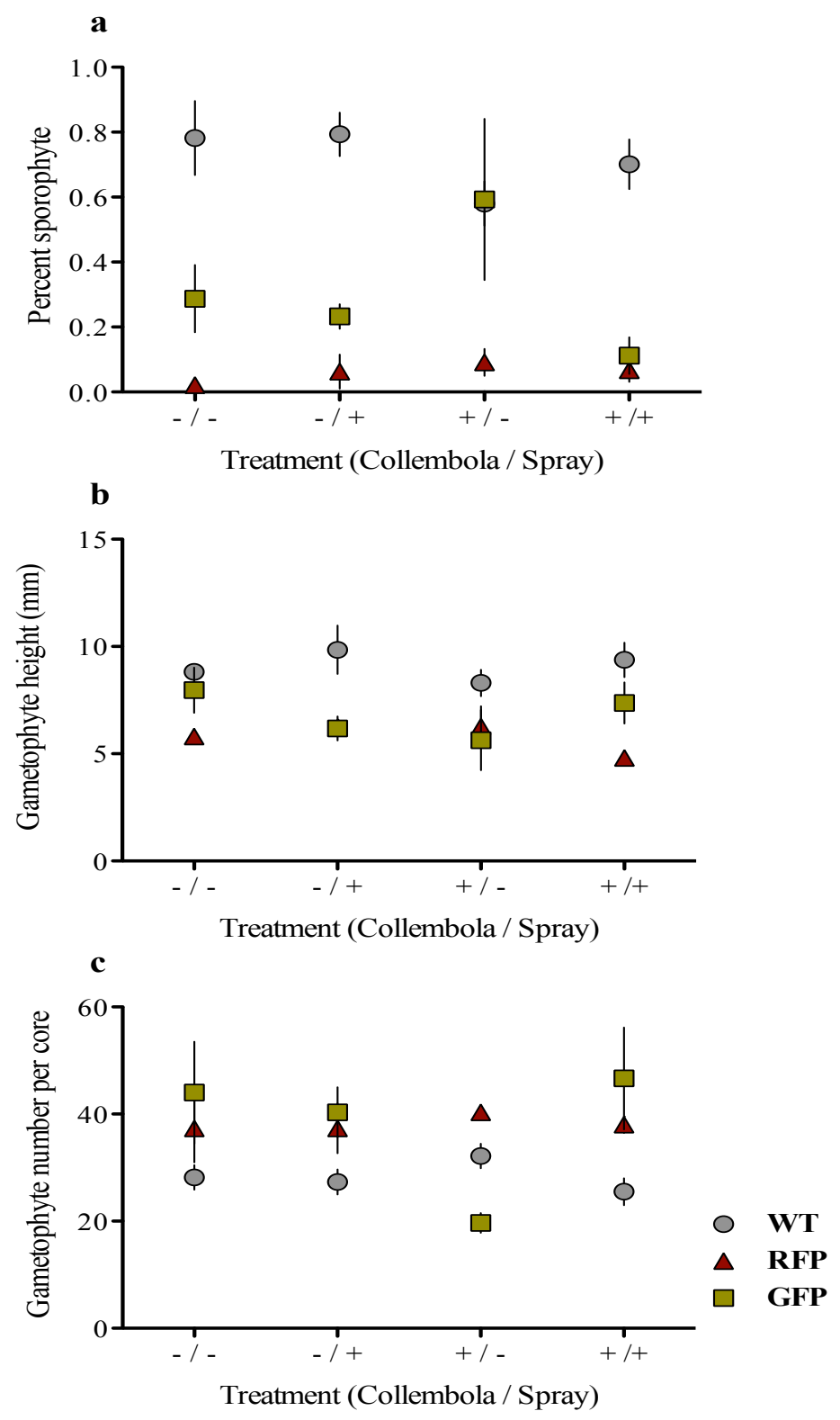


\section{Appendix C}

\section{Testing mutualism in two of Earth's most ancient lineages: the mosses and microarthropods}

\section{Introduction}

Pollination syndromes have been a subject of intense interest for naturalists and scientists since the mid-late 1700's when pioneers such as Klreuter, Sprengel, and shortly thereafter, Darwin, began to intensely study interactions between flowers and their pollinators (reviewed in Fenster et al., 2004). Theories concerning pollination biology have generally been formulated around angiosperms, and empirically tested on angiosperm systems. In particular, a great deal of literature has been developed to explain and test how the mutualistic relationship between plants and their pollinators has shaped characters and phylogenies of both players (Thompson \& Cunningham, 2002, Waser et al., 1996) as well as what factors act to maintain the intricate dance of plant-animal mutualism (Bascompte et al., 2006, Bronstein et al., 2006).

The upper Ordovician ( 450Mya) represents a time of great evolutionary importance where fossil records place the radiation of the land plants as well as that of the terrestrial arthropods (Cloudsley-Thompson, 1988, Goffinet, 2009). Often overlooked by plant mating and breeding system biologists, the bryophyte system presents a unique opportunity to 
apply traditional angiosperm theory on a novel and potentially primary 'plant-pollinator' relationship. An exploration of a non-angiosperm "pollination" system would allow us to begin asking plant-pollinator theory questions in a system in which the theory was not developed.

As an ever present reminder of the aquatic origins of sex, sexual reproduction in bryophytes requires that free-motile sperm 'swim' with the aid of water across the terrestrial landscape to fertile females (Muggoch \& Walton, 1942, Paolillo, 1981). This model of 'swimming sperm' has led to the general view that sperm dispersal within bryophytes is limited, with most fertilization occurring within about $10 \mathrm{~cm}$ (Longton, 1976, Shaw, 2000). Nevertheless, this view of moss reproduction comes under scrutiny as research indicates that mosses maintain high genetic variability (Shaw, 1991, Van der Velde \& Bijlsma, 2000), exhibit prevalent multiple paternity while having an apparent heterozygote advantage in out-crossed sporophytes of species with separate sexes (Eppley et al., 2007, Szovenyi et al., 2009), and that gamete dispersal rates are high and dispersal distances are least an order of magnitude farther than previously believed (Bisang et al., 2004, Van der Velde et al., 2001). Furthermore, recent research demonstrates that moss sperm can be dispersed by the common moss-microarthropods: mites and Collembola (Cronberg et al., 2006, Rosenstiel et al., 2012). This is an idea supported by early, often overlooked work by researchers suggesting that arthropods act as ecologically relevant sperm transport vectors (Muggoch \& 
Walton, 1942). Taken together, these studies reveal a need to reevaluate the complexity and drivers of sexual reproductive success in dioicous mosses.

Plant-pollinator syndromes reveal a variety of rewards or benefits that partners may (or not) gain from the relationship, often revealing if a true mutualism is at work or instead, a facultative, non-reward based relationship. We tested the basic tenets of plant-pollinator theory using the common moss, Ceratodon purpureus and Collembola and found a significant relationship between them, where the Collembola differentially responded to sex-specific volatile cues emitted mostly from female mosses (Rosenstiel et al., 2012). In turn, the microarthropods are enhancing sexual reproductive success in mosses as more sporophytes were produced when Collembola were included in microcosms. These data combined demonstrate a "pollination"-like syndrome for mosses, yet little is known about the fitness benefits for either player in this potential mutualism nor the cascade effects and influences acting to maintenance this relationship. In fact, no studies to my knowledge have tested genetic variation and dispersal potential in mosses as it relates to the ubiquitous microarthropod inhabitants of the moss canopies (Andrew et al., 2003b, Gerson, 1969).

The small stature and easily tractable bryophytes present an ideal system by which to base ecosystem-level studies. Mosses are easily replicated in experimental settings without losing many 'natural' features or characters as demonstrated by studies that focus on nutrient cycling, multi- 
trophic interactions, and habitat fragmentation (e.g., Lindo and Gonzalez 2010, Staddon et al. 2010, Chisholm et al. 2011(Srivastava et al., 2004).

In this study I aim to use the tractability of the moss system to explore the fitness consequences for mosses engaged in this novel "pollination system" by testing the mutualistic relationship of plants and insects in the primary group of terrestrial plants, the bryophytes. Here I test the hypothesis that microarthropods are facilitating elevated genetic variation in moss populations through increased sperm dispersal as they move about the moss canopies. I predict that the maintenance on this relationship is reliant on a multi-tiered community of players and that existing genetic variation in male moss fitness traits in combination with microarthropod vectors will result in differential successes as well as diversity (as shown by magnitude of genetically different sporophytes) in the mosses. I ask how the addition of moss dwelling microarthropods affects moss fitness specifically by: measuring relative sporophyte production, sperm dispersal distance, genetic diversity, male fitness characters, canopy structure, sporophyte height, photosynthetic capacity, nutrient levels and ultimately community-wide dynamics in custom grown bryospheres.

\section{Materials and Methods}

Ceratodon purpureus (Hewd) was collected from two naturally occurring populations in Portland, Oregon and one in North Plains, Oregon in 2010. 
Each of the three populations were located a minimum of $9 \mathrm{~km}$ apart. Single gametophytes of mosses from each population isolated and identified by sex under a microscope (Leica MZ9 stereo-microscope). All individuals were finely chopped and planted separately in $6.35 \mathrm{~cm}^{2}$ pots on a mix of sand and peat moss (2:1), and grown in a common garden greenhouse. This process was repeated until many pots of the same individuals were growing simultaneously.

\section{Male moss identity}

We wanted to be able to definitively identify each potential male moss father of the fertilized females upon sporophyte production. To do so we used several primers for microsatellites have already been developed for $C$. purpureus, (Clarke et al., 2009) and DNA sequences are available from GenBank. To identify each male we used four microsatellite primers to obtain a distinct fingerprint of each male. Genomic DNA of individual $C$. purpureus gametophytes from each male (at least 6 replicates from same and different pots) was extracted and analyzed using microsatellites developed to assess worldwide C. purpureus variation (Clarke et al., 2008). DNA extractions were conducted using a high-throughput system for rapid extraction and amplification of genomic DNA by use of Plant REDExtract- $N$ Amp kit components (Sigma-Aldrich, USA) and using a modified version of manufacturer protocols. DNA extractions were accomplished by placing a 
single gametophyte in $20 \mu \mathrm{L}$ of extraction solution (Sigma) and briefly disrupting the tissue using pipette tips. Samples were incubated (Applied Biosystems Thermocycler 2720) at $65^{\circ} \mathrm{C}$ for 10 minutes (m), followed by $10 \mathrm{~m}$ at $95^{\circ} \mathrm{C}$ and then held at $4^{\circ} \mathrm{C} .20 \mu \mathrm{L}$ of neutralization solution is then added to the incubated samples, vortexed briefly and used immediately for amplification or kept at $4^{\circ} \mathrm{C}$ until use with REDExtract-N-Amp PCR Ready Mix. We used 5' forward (FAM labeled) and reverse primers (MWG Eurofins Operon, Germany) as described in Clarke et al. 2008. Of five previously published markers, four reliably amplified the desired DNA regions (CEPU 105, 108,109 and 111(Clarke et al., 2008)).

For a $10 \mu \mathrm{L}$ reaction, $5 \mu \mathrm{L}$ REDE-mix, $0.5 \mu \mathrm{L}$ of both forward and reverse primers ( $0.5 \mu \mathrm{M}$ final concentration), $3 \mu \mathrm{L}$ of RNAase free sterile water and $1 \mu \mathrm{L}$ of DNA template. DNA was amplified with Applied Biosystems Thermocycler 2720 with conditions as follows: $10 \mathrm{~m}$ at $94^{\circ} \mathrm{C}$, followed by 35 cycles of: $30 \mathrm{~s}$ at $94^{\circ} \mathrm{C}, 30 \mathrm{~s}$ at $57^{\circ} \mathrm{C}, 45 \mathrm{~s}$ of $72^{\circ} \mathrm{C}$, and followed by $10 \mathrm{~m}$ at $72^{\circ} \mathrm{C}$ and held at t $4^{\circ} \mathrm{C}$ until use or storage at $4^{\circ} \mathrm{C}$. For CEPU 111 the hold at $72^{\circ} \mathrm{C}$ was extended to $20 \mathrm{~m}$ to allow ample time for the final addition of the adenosine nucleotide, our preliminary analyses indicated the extension time was necessary to confidently identify fragment lengths for this particular amplified region. PCR products were tested for DNA using 1.5\% agarose gel in $1 \mathrm{x}$ TAE Buffer. Gels were run at $90 \mathrm{v}$ for $50 \mathrm{~m}$, visualized on a Typhoon scanner to verify primer adequacy (by FAM fluorescence) and then 
verified by ethidium bromide or SYBR green staining and visualized and photographed using a transilluminator. Samples were kept at $4^{\circ} \mathrm{C}$ until $1 \mu \mathrm{L}$ per sample was processed by the Center for Genome Research and Biocomputing Core Labs at Oregon State University (Corvallis, OR, USA) for sequencing via an $\mathrm{AB} 3730$ capillary DNA sequencer to resolve fluorescently labeled DNA fragments for genotyping applications. Rox40 internal standard was used to score alleles (bp) using Peak Scanner Software (Applied Biosystems).

\section{Bryosphere propagation}

Females. Replicates of three female individuals (from previously described cloned pots) of each of three populations were cut from the green gametophytes to equal $4 \mathrm{~g}$ per individual, $12 \mathrm{~g}$ per population, combined to a total $36 \mathrm{~g}$ of female moss tissue. The tissue was sifted by hand to mix the populations and homogenized (Cole Parmer) in $25 \mathrm{ml}$ tap water in small batches. All blended tissue was combined to make a final homogeneous female moss solution and then divided into $1650 \mathrm{ml}$ Falcon tubes of female solution, one per array.

Males. Replicates of the previously genotyped individual males (two from each of three populations) were cut from pots ending in $4 \mathrm{~g}$ of each individual male. Two groupings of males, each with one male of each population were then deemed Males A or Males B. Male tissue of each grouping was 
combined, mixed and homogenized in small batches, resulting in two $50 \mathrm{~mL}$ Falcon tubes, each containing 12g of mixed male tissue, either Males A or B.

\section{Bryospheres.}

Sixteen $20 \mathrm{G}$ plastic pots of $43 \mathrm{~cm}$ diameter were used for the bryospheres. All pots were filled with a blend of sand and peat moss (2:1) and thoroughly watered until all substrate was well saturated. Since the mosses do not grow roots, the pots were intentionally large to provide adequate buffering from excessive cold and drought (tested previously). A plastic cap of $10.5 \mathrm{~cm}$ diameter was placed in the center of each pot, covering the center of the pot during application of the females.

Female solution was spread evenly across the pot surface in a crisscross pattern using a syringe (except for where the plastic core was placed). The process was repeated in all bryospheres.

The plastic cap was removed from the center of each pot. Half of the arrays received $10 \mathrm{~mL}$ of Males A solution and the other half $10 \mathrm{~mL}$ of Males B in the $4.3 \mathrm{~cm} \mathrm{~d}$ center of the bryospheres. The male moss was applied with a syringe. Male concentration was higher than that of females per area by about $2 \mathrm{x}$.

Pots were kept in the greenhouse and hand watered with a fine-mist nozzle heard two times per day for the first two months of growth. Mosses were kept at constant light temperature $\left(18^{\circ} \mathrm{C}\right)$ and a fourteen-hour 
photoperiod with lights levels $\sim 200 \mu \mathrm{E}$ and a nighttime temperature of $10^{\circ} \mathrm{C}$. Open Top Chamber rings constructed of Sun-Lite ${ }^{\circledR}$ (Solar Components Corporation, NH, USA) fiberglass solar glazing material that transmits full spectrum sunlight, were placed around the top of each pot. The rings slightly tapered at the open end to a $35.6 \mathrm{~cm}$ diameter and the base of the ring fit snugly into the bryosphere pots with a $40.4 \mathrm{~cm}$ base. The rings served as a barrier to prevent excess invertebrate immigration or emigration as well as to test OTC rings for a simultaneous bryosphere warming experiment. After a uniform mat of gametophytic growth was detected in all pots, the bryospheres were moved outside (February, 2013). Once outside the pots were lifted $30 \mathrm{~cm}$ from the concrete patio ground by plastic trays allowing for the pots to drain if needed and to further prevent invertebrate movement into the pots from the ground. There were no overhanging branches or trees and the pots were separated from other mosses or grasses by at least $20 \mathrm{~m}$. A large building or tall conifer trees shadowed the pots for approximately half of every day. Pots were watered three times per week to supplement rainfall when necessary; otherwise the bryospheres experienced natural Portland, OR conditions.

\section{Microarthropod additions}

Natural populations of moss-dwelling invertebrates were added to bryospheres by collecting moss patches from the Portland, and North Plains, 
OR regions. C. purpureus patches were used for the majority of the additions, other co-occurring mosses were used when necessary. Patches were collected into a plastic bag and immediately brought back to the greenhouse, weighed into nine $100 \mathrm{~g}$ rations, misted with tap water and added to modified collapsible Berlese funnels for live extraction via humidity and light gradient (Andrew et al., 2003a, Macfadyen, 1953, Walter, 1987, Yanoviak et al., 2004). A custom built tent was designed for live extractions to occur without moving the bryospheres from their outside location. The tent was moved over the entire bryosphere array to avoid a treatment effect and funnels were suspended over the bryospheres that were designated for invertebrate additions. The $15 \mathrm{w}$ incandescent light bulbs used in each funnel were suspended high enough above the bryospheres that there was no heat added to the bryospheres. Invertebrates were live extracted for 48 hours after which the tent was removed from over all bryospheres. One control extraction into an ethanol solution was conducted with all extractions into bryospheres allowing for a representative sample of the abundance and composition of each invertebrate addition. Nine extractions were conducted over the course of one year. Dried mosses were weighed and returned to the local environment. 


\section{Canopy physiology}

In February of 2014, one year after bryosphere establishment sporophytes had begun to develop in seven of 16 bryospheres. Before more sporophyte development, canopy measures were taken on leafy gametophytic moss cover. We determined bryosphere moss canopy chlorophyll content by chlorophyll fluorescence (Gitelson et al., 1999) using a hand-held meter, (Opti-Sciences, CCM-300 Chlorophyll Content Meter, Hudson NH, USA), using standard manufacturer recommended protocols, five values per bryosphere were averaged to obtain one data point per bryosphere. To non-invasively assess chlorophyll fluorescence parameters we measured maximum quantum yield of PSII (Fv/Fm). Fluorescence was measured on dark-adapted C. purpureus at five locations in each bryosphere before sunrise (Bilger et al., 1995, Proctor, 2010). Chlorophyll fluorescence was measured with an OSp5 portable chlorophyll fluorometer (Opti-Sciences, Hudson, NH) using standard protocols, and fluorometer settings were chosen to optimize the fluorescence response during the various measurement efforts.

\section{Sporophyte collection and location}

After 15 months (May 2014), twelve of sixteen bryospheres had developed sporophytes, some pots with hundreds. Sporophytes were collected in three phases as they matured. Mature sporophytes were identified as having fully developed and reddened calyptra that had turned perpendicular to the seta. 
Each sporophyte that developed was surveyed for distance from center and angle vector, pulled from the bryosphere with forceps along with its maternal gametophyte if possible. Each sporophyte's height was recorded and placed into a conical tube with the maternal tissue for future genetic analyses.

\section{Data Analysis}

Mixed-model ANOVA was performed on both sporophyte distance and from center and sporophyte height by treatment and Male genotype mix, and the interactions between them. T-tests were conducted to discern differences between treatments in CFR and Fv/Fm. Sporophyte height was regressed against distance from center. All statistical analyses were performed using JMP 10 (SAS Institute, 2012).

\section{Results}

\section{Male moss identity}

Each male was identified by one to three microsatellite region alleles. We found within and among population variation in all three populations. Males have subsequently been numbered Male 1-6, with individual Males 1-3 of group A, and 4-6 of group B. All replicated individuals displayed repeated allelic identities therefore were successfully identified as identifiable potential fathers of the sporophytes. 


\section{Microarthropod additions}

Microarthropod extractions were conducted nine times over 15 months. Each extraction also had a paired control extraction. An average $\sim 400$ microarthropods (per bryosphere) were extracted during each extraction event. The majority of invertebrates are composed of Collembolans and oribatid mites, but most all other major invertebrate groups were represented including: Diptera, Coleoptera, Hymenoptera, Archnida and Annelida.

\section{Canopy physiology}

Chlorophyll content (reported as Chlorophyll Fluorescence Ratio, CFR) was measured in February when sporophytes first began growing and in May after sporophyte harvest, and in both time points CFR was slightly higher though not significantly so in the bryospheres without microarthropods (Fig. A.C1; $\mathrm{t}=0.22 ; \mathrm{P}=0.82$ in February and $\mathrm{t}=0.71 ; \mathrm{P}=0.49$ in May; $\mathrm{N}=16$ ). Similarly, Fv/Fm values were also slightly higher in bryospheres without microarthropods added, but not significantly so (Fig. A.C2; t=0.85; P=0.40).

\section{Sporophytes}

Of the sixteen bryospheres, sporophytes were collected from thirteen. The rest did not develop sporophytes. Of the thirteen, eight were bryospheres with added microarthropods and the sporophyte production varied significantly between treatments $(\mathrm{t}=6.60 ; \mathrm{P}<0.0001 ; \mathrm{N}=1067) .839$ 
sporophytes were collected from bryospheres with invertebrates, and 228 from bryospheres without the invertebrate treatments (Fig. A.C3). Mean sporophyte distance from center was significantly greater in bryospheres without added invertebrates (Fig A.C4a; $\mathrm{t}=6.70 ; \mathrm{P}<0.0001 ; \mathrm{N}=1067$ ). Mean sporophyte distance from males center without invertebrates was $13.61 \mathrm{~cm} \pm$ $0.29 \mathrm{SE}$, and with invertebrates $\mathrm{N}=839$; mean distance from center $11.42 \mathrm{~cm}$ $\pm 0.15 \mathrm{SE}$. Sporophyte height was also significantly different between invertebrate treatments (Fig. A.C4b t=3.20; $\mathrm{P}=0.0014 ; \mathrm{N}=1067$ ). Mean sporophyte height was greater in bryospheres with added invertebrates $(22.80 \mathrm{~mm} \pm 0.14 \mathrm{SE})$ than without $(21.85 \mathrm{~mm} \pm 0.26 \mathrm{SE})$. Male group A fathered significantly more sporophytes (762) than did male group B (305 sporophytes). This occurred in both treatments (Fig. A.C5). We also found a significant positive correlation between distance from center and sporophyte height (Fig. A.6; F=36.05; $\mathrm{P}<0.0001$ ). We found that male genotype was not a predictor of sporophyte distance from center alone, but it was when in interaction with treatment (with or without microarthropods), and, treatment as well as the interaction between treatment and male genotype were also significant predictors of sporophyte height (Table A.C1).

\section{Continuing work}

Paternity and genetics. All individuals used in the bryospheres (6 males, 9 females) are currently being grown in axenic culture for genetic sequencing. 
The haploid father of each sporophyte will be derived through identification of maternal gametophyte and subtraction of that from the attached diploid sporophyte. Upon subtracting the maternal haploid genotype from the diploid sporophyte genotype, paternity can be derived by comparison of deduced paternal genotype to already genotyped males. Further, the spores from the sporophytes will be isolated, counted and germinated providing a direct measure of fitness above and beyond sporophyte production (McDaniel et al., 2007). These data, once collected will provide us with the first assessment of moss genetic diversity as it relates to interactions with invertebrates as well as a unique opportunity to understand male competitive ability, and fitness traits. Further, male and female individual C. purupreus genomes have recently been sequenced. C. purpureus has chromosomally derived sex determination and much of the genome is located on the sex chromosome (McDaniel et al., 2013b) given this experimental set-up we plan to begin identifying the genetic origins of sexually dimorphic male and female fitness traits.

\section{Community and canopy measures.}

We will identify the fungal community related to each bryosphere by ergosterol quantification and fungal functional group identification by TRFLP analysis. We will also conduct 454-sequencing on the $16 \mathrm{~S}$ ribosomal subunit to identify bacterial OTUs. We predict that there will be differences 
in the bryospheres in microbial assemblages as it relates to added microarthropods as well as by male genotype grouping as well as spatially between the moss sexes.

Passive flow canopy respiration rates will be quantified using IRGA LI-6400 (LI-COR, USA) in a custom-built bryosphere respiration chamber. The same chamber will that will also be used to sample the headspace volatile organic compounds (VOCs) collected over the canopy onto an analytical thermal desorption (ATD) cartridge and subsequently analyzed via a LECO Pegasus 4D GC×GC-TOFMS system (LECO, St. Joseph, MI, USA), These data will allow us to determine the relative influence of microarthropods and genotype on canopy-level VOCs. Finally, a nutrient analysis will be conducted on moss tissue and shallow soil, minimally for carbon and nitrogen and potentially for full nutrient and metal analysis.

\section{Conclusions and Significance}

These data distinctly show that in a simulated ecosystem, the addition of naturally occurring invertebrates to $C$. purpureus moss arrays increases sporophyte production in bryospheres that had invertebrates added. These results were more pronounced than we could have predicted (Fig. A.C3). Due to the extreme treatment effect (with or without added invertebrates) in our bryospheres, we will be performing the aforementioned further analyses in hopes to obtain a comprehensive understanding of the factors influencing 
this system. We saw no differences in canopy physiology between treatments which indicates that in this first year, the mosses have comparable photosynthetic capacity, yet we predict that as the bryospheres mature, there may be more pronounced differences that arise due to shifts in nutrient availability. These early results have interesting implications concerning individual male fitness traits, in particular that the Males A group fathers more sporophytes in both treatments than Males B (Fig. A.C5), and that the interaction between males and treatment are significant, implying that perhaps certain males are better adapted to microarthropod-mediated transport than others. Also interesting is that sporophyte heights are significantly correlated with the distance from the male center (Fig. A.C6). Future work into the possible influences on sporophyte height including photosynthetic capacity and water relations is much needed as data on these energetic transfers is generally lacking in the literature (Ligrone et al., 2012).

Through investigation into the respective roles of, and potential benefits for both the moss and invertebrate partners in this prospective mutualism, we will better understand the fundamentals of the ubiquitous plant-insect interactions and the factors that maintain it. Here we begin the investigation with an in-depth analysis of the effects of microarthropods on mosses, in particular on moss fitness. Upon completion of the proposed experiment, we will have a greater understanding of how mossmicroarthropod interactions have shaped the ecology, evolution and 
functional dynamics of the moss ecosystem and have data by which to assess the compelling idea that mosses and microarthropods may represent one of the earliest, and unexplored, plant-animal fertilization mutualisms.

To our knowledge, this study will be the first to reveal whether genetic variation among moss populations can be attributed to male gamete dispersal as facilitated by microarthropod vectors. Investigation into what the plant partner may be gaining from such an interaction could help answer an unresolved question in plant biology, namely, why after 450 million years, do such a high percentage of mosses maintain the ancestral state of separate sexes (about $60 \%$ vs. $6 \%$ in angiosperms). This study will also begin to reveal potential reasons that mosses emit sex-specific volatile cues and the significance of such cues for moss-dwelling microarthropods. Why would these microarthropods be attracted to sex-specific volatile cues emitted from female mosses? Do these patterns have parallels with sex-specific volatile cues and sex-specific rewards for pollinators as in the angiosperm system? Understanding the fitness consequences of this non-angiosperm "pollination" system will greatly broaden our understanding of the diversity of "pollinator" interactions within the plant kingdom and support the development of an emerging novel system for use in future tests of "pollination" theory as well as genetic basis for fitness traits and maintenance on mutualism and community-wide processes. 


\section{Acknowledgements}

We would like to thank the National Science Foundation, Doctoral

Dissertation Improvement Grant (no. 1210957, awarded to S. Eppley and E. Shortlidge) for providing funding this project and to the PSU Research Greenhouse facilities. We also thank: S.C. Woll, T. Schroyer, E. Blue, T. Deakova, T. Williams for laboratory help, M. Balkan for ongoing microbial analyses, and the S. McDaniel lab for extended collaboration underway for this ongoing project. 


\section{Tables and Figures}

Table A.C1. Results of mixed-model ANOVA and post hoc Tukey's HSD analyses on sporophyte distance from center and sporophyte height as they relate to male moss individual groups (A or B) and invertebrate treatments (+ or -).

\begin{tabular}{|c|c|c|c|c|c|c|c|c|}
\hline $\begin{array}{c}\text { Distance from } \\
\text { Center }\end{array}$ & $\begin{array}{c}\text { Estima } \\
\text { te }\end{array}$ & $\begin{array}{l}\text { Std } \\
\text { Error }\end{array}$ & $\begin{array}{c}\mathbf{t} \\
\text { Ratio }\end{array}$ & $\begin{array}{c}\text { Prob }>1 \\
t \mid\end{array}$ & $\begin{array}{l}\text { Tukey's } \\
\text { HSD }\end{array}$ & & & $\begin{array}{l}\text { Least } \\
\text { Sq } \\
\text { Mean }\end{array}$ \\
\hline Intercept & 12.08 & 0.30 & 40.53 & $<.0001$ &,$- \mathrm{A} \quad \mathrm{A}$ & & & 13.80 \\
\hline Male Genotype (A B) & 0.11 & 0.30 & 0.38 & 0.7031 &,$+ \mathrm{B} \quad \mathrm{A}$ & & & 13.02 \\
\hline \multicolumn{9}{|l|}{ Invertebrate } \\
\hline Treatment (+ -) & 0.28 & 0.30 & 0.94 & 0.3473 &,$- \mathrm{B} \quad \mathrm{A}$ & & B & 10.92 \\
\hline \multirow[t]{3}{*}{ Treatment*Genotype } & 1.33 & 0.30 & 4.46 & $<.0001$ &,$+ \mathrm{A}$ & & B & 10.59 \\
\hline & \multicolumn{4}{|c|}{$\mathbf{t}$} & \multicolumn{3}{|l|}{ Tukey' } & Least \\
\hline & $\begin{array}{c}\text { Estima } \\
\text { te }\end{array}$ & $\begin{array}{l}\text { Std } \\
\text { Error }\end{array}$ & $\begin{array}{c}\text { Rati } \\
\text { o }\end{array}$ & $\begin{array}{c}\text { Prob }>1 \\
t \mid\end{array}$ & $\begin{array}{l}\text { S } \\
\text { HSD }\end{array}$ & & & $\begin{array}{l}\text { Sq } \\
\text { Mean }\end{array}$ \\
\hline Intercept & 21.90 & 0.28 & 79.62 & $<.0001$ &,$+ \mathrm{B} \quad \mathrm{A}$ & & & 23.03 \\
\hline Male Genotype (A B) & 0.43 & 0.28 & 1.57 & 0.1173 &,$+ \mathrm{A} \quad \mathrm{A}$ & $\mathrm{B}$ & & 22.66 \\
\hline Invertebrate & & & & & & & & \\
\hline Treatment (+ -) & -0.95 & 0.28 & -3.47 & 0.0005 &,$- \mathrm{A}$ & $\mathrm{B}$ & $\mathrm{C}$ & 21.99 \\
\hline Treatment* Genotype & 0.62 & 0.28 & 2.24 & 0.0251 &,$- \mathrm{B}$ & & $\mathrm{C}$ & 19.89 \\
\hline
\end{tabular}


Figure A.C1 Chlorophyll content from bryospheres at two time points. $(\mathrm{N}=16)$

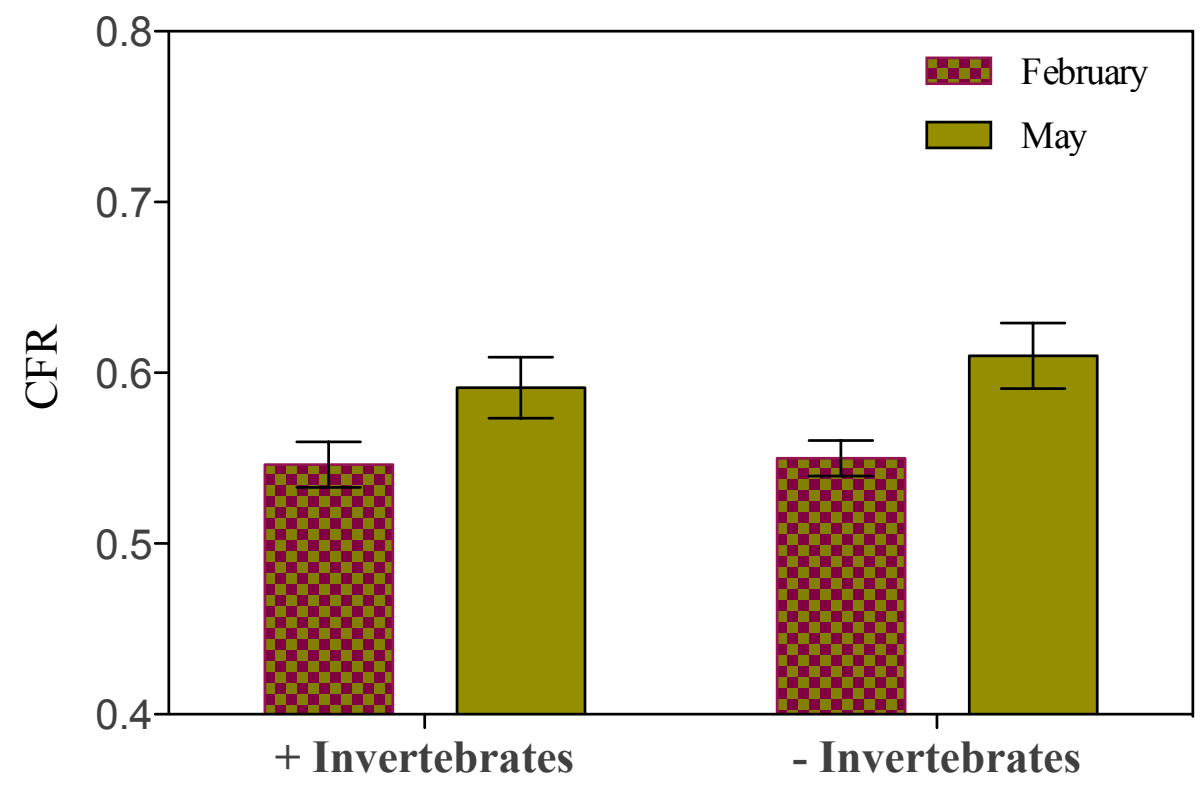


Figure A.C2 Fv/Fm values of C. purpureus canopies after one year of growth in both treatments $(\mathrm{N}=16)$.

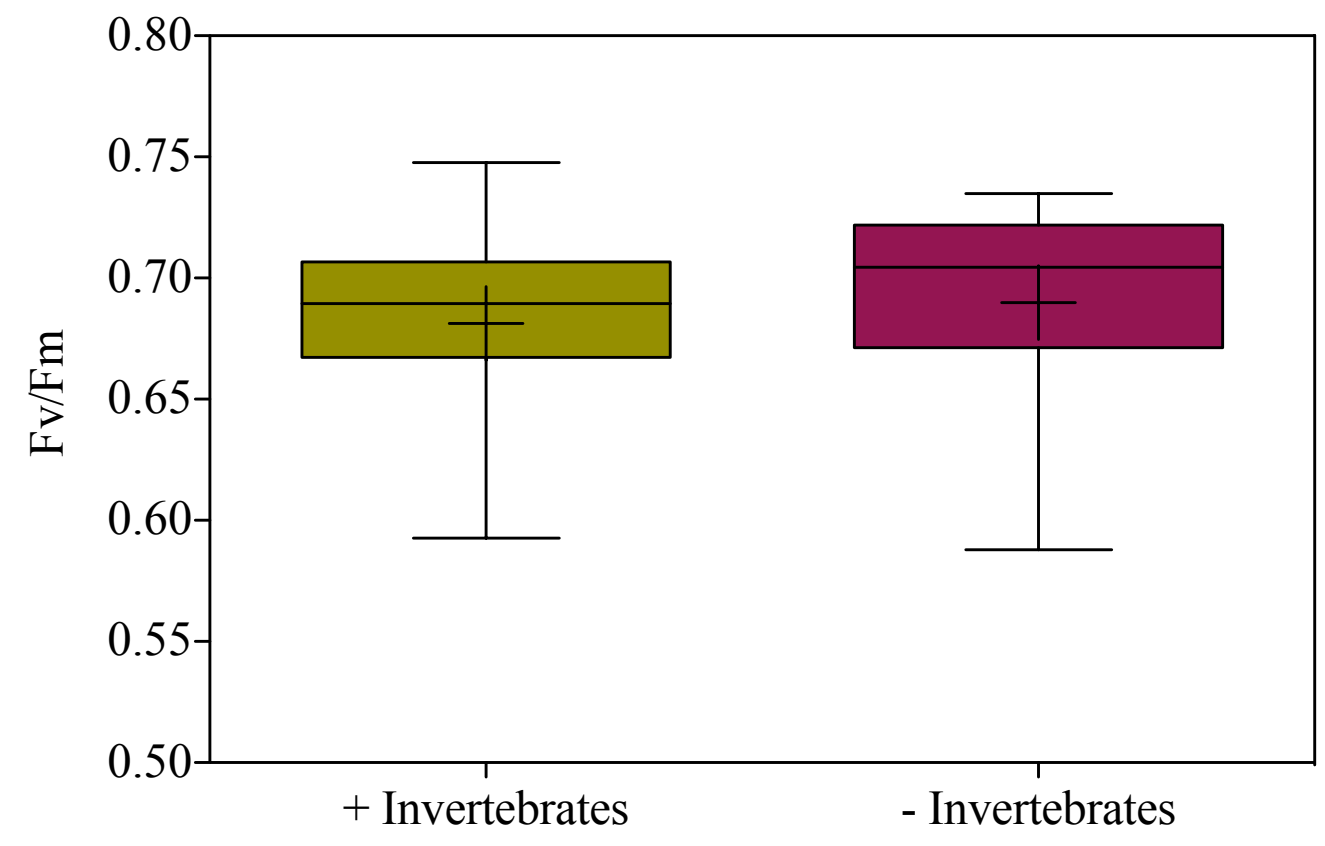


Figure A.C3 Total sporophytes produced in bryospheres after 16 months ( $\mathrm{N}=1067 ; 839$ in +Invertebrates, 228 in -Invertebrates).

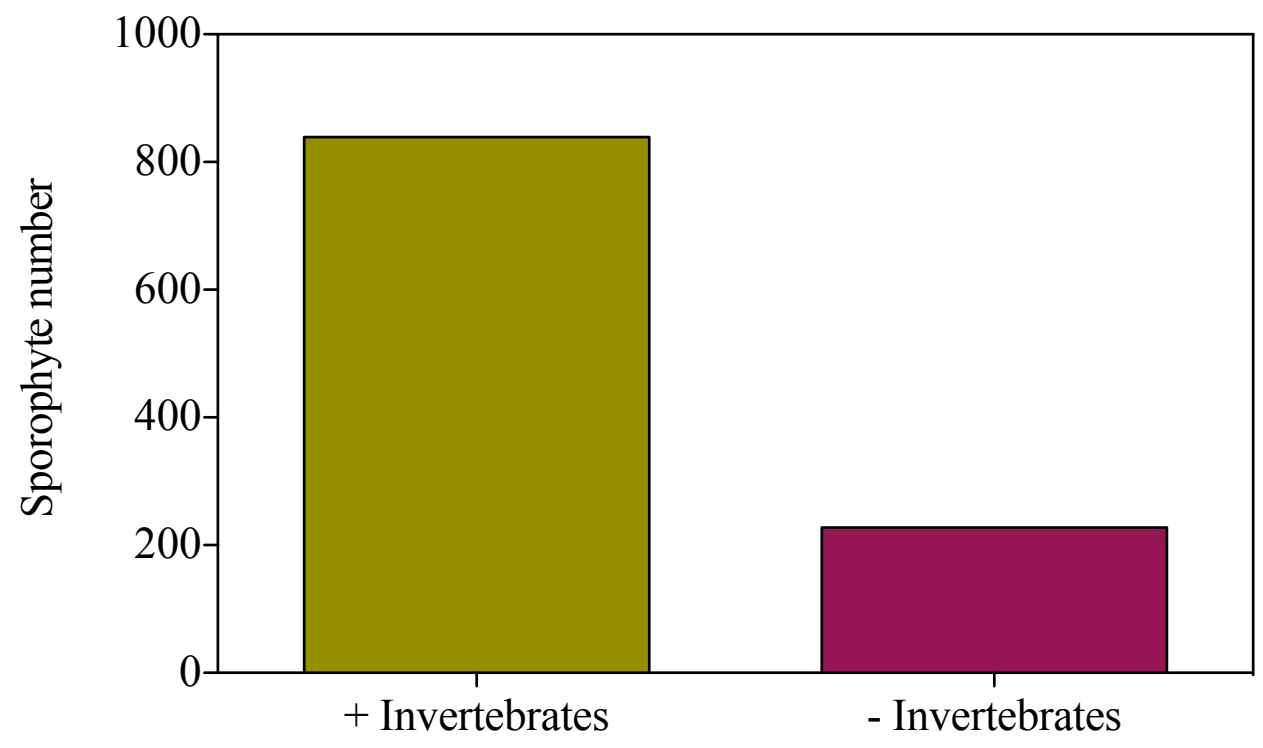


Figure A.C4 Sporophytes in bryospheres. a) Each sporophyte's measured distance from center - bryospheres without invertebrates had a greater mean sporophyte distance from center ( $\mathrm{t}=6.70 ; \mathrm{P}<0.0001 ; \mathrm{N}=1067)$, b) Each sporophyte's height - bryospheres with invertebrates' sporophytes were taller $(\mathrm{t}=3.20 ; \mathrm{P}=0.0014 ; \mathrm{N}=1067)$.
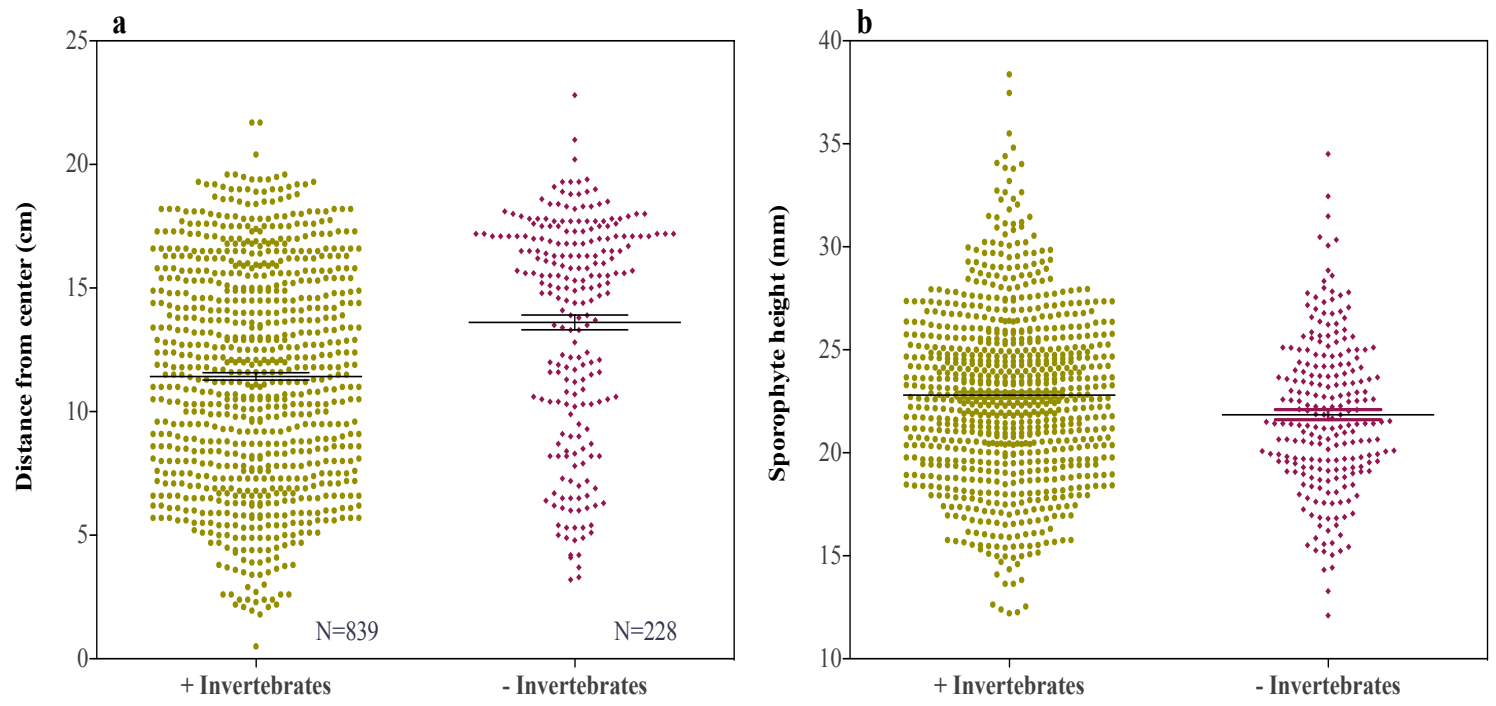
Figure C5 Sporophytes produced by each male grouping - Males A (762 sporophytes) or B (305 sporophytes).

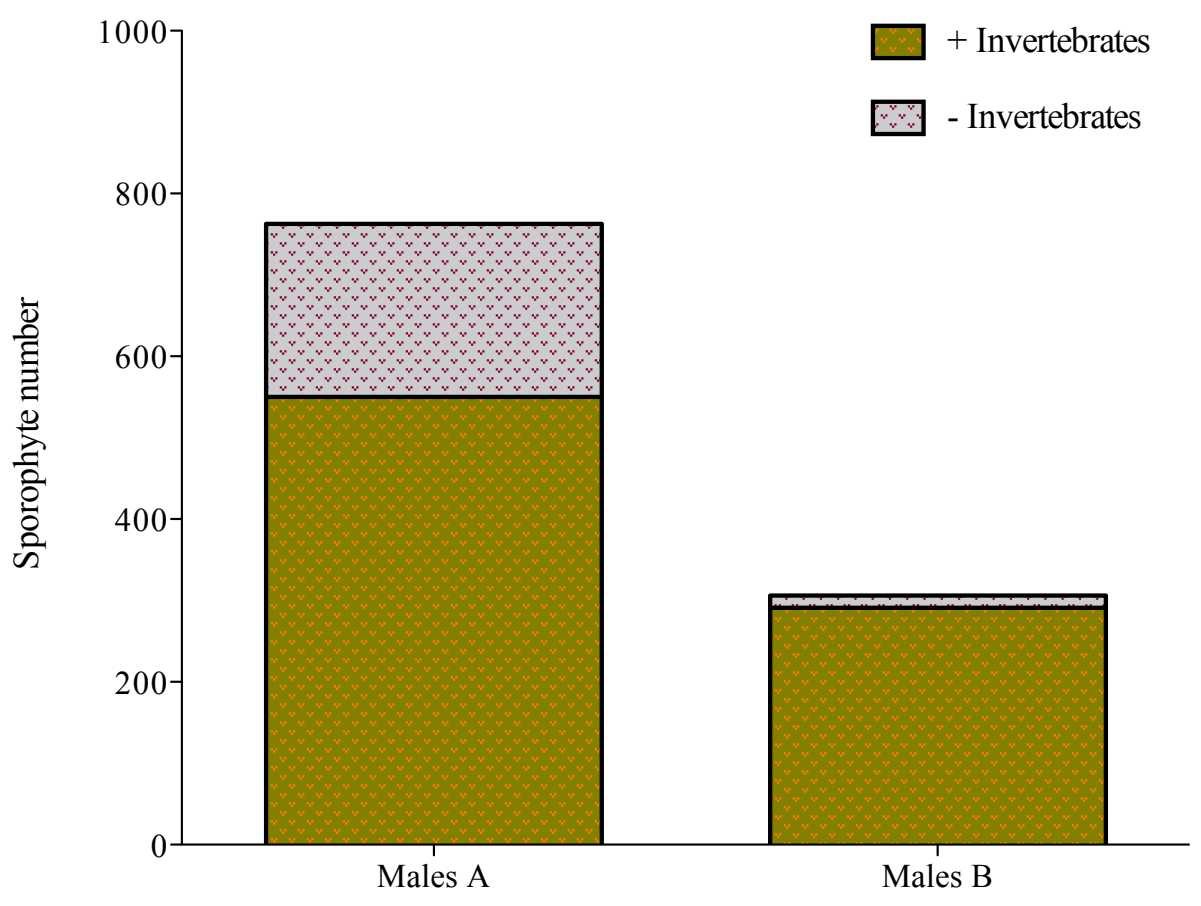


Fig. A.C6 Correlation between sporophytes distance from center and sporophyte height. The further away from center, the taller the sporophyte $(\mathrm{P}<0.0001)$.

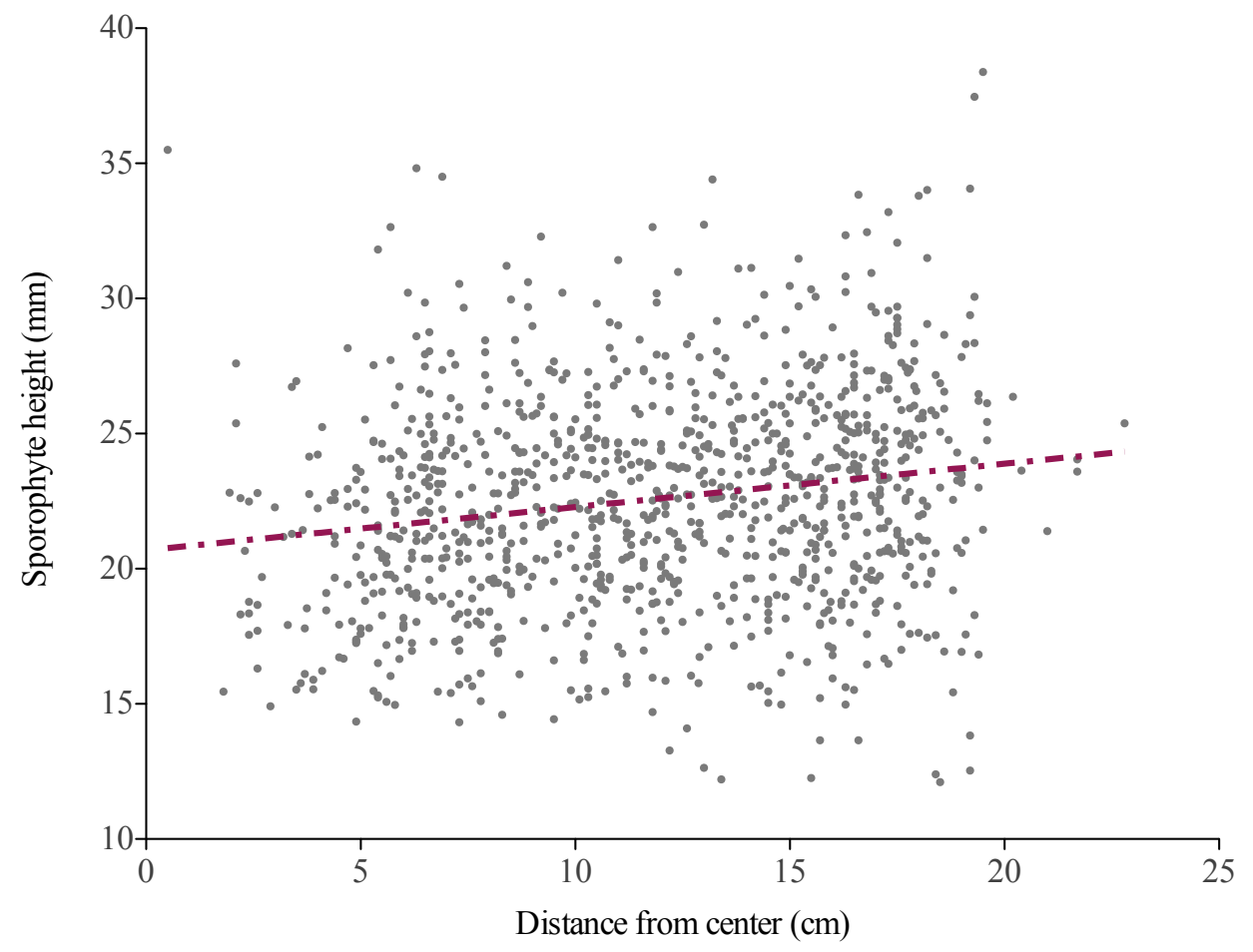




\section{Appendices References}

Andrew N, Rodgerson L, Dunlop M (2003a) Variation in invertebratebryophyte community structure at different spatial scales along altitudinal gradients. Journal of Biogeography, 30, 731-746.

Andrew NR, Rodgerson L, Dunlop M (2003b) Variation in invertebratebryophyte community structure at different spatial scales along altitudinal gradients. Journal of Biogeography, 30, 731-746.

Ashman TL (2009) Sniffing out patterns of sexual dimorphism in floral scent. Functional Ecology, 23, 852-862.

Bascompte J, Jordano P, Olesen JM (2006) Asymmetric coevolutionary networks facilitate biodiversity maintenance. Science, 312, 431-433.

Bilger W, Schreiber U, Bock M (1995) Determination of the quantum efficiency of photosystem II and of non-photochemical quenching of chlorophyll fluorescence in the field. Oecologia, 102, 425-432.

Bisang I, Ehrlen J, Hedenas L (2004) Mate limited reproductive success in two dioicous mosses. Oikos, 104, 291-298.

Bragina A, Berg C, Müller H, Moser D, Berg G (2013) Insights into functional bacterial diversity and its effects on Alpine bog ecosystem functioning. Scientific reports, 3.

Bronstein JL, Alarcón R, Geber M (2006) The evolution of plant-insect mutualisms. New Phytologist, 172, 412-428.

Charlesworth D (1985) Distribution of dioecy and self-incompatibility in angiosperms. Evolution: Essays in honour of John Maynard Smith, 237-268.

Clarke LJ, Ayre DJ, Robinson SA (2008) Somatic mutation and the Antarctic ozone hole. Journal of Ecology, 96, 378-385.

Clarke LJ, Ayre DJ, Robinson SA (2009) Genetic structure of East Antarctic populations of the moss Ceratodon purpureus. Antarctic Science, 21, 51-58.

Cloudsley-Thompson JL (1988) Palaeontology and Phylogeny. In: Evolution and Adaptation of Terrestrial Arthropods. pp 1-9. Springer.

Cove D (2005) The moss Physcomitrella patens. Annu. Rev. Genet., 39, 339358.

Cove DJ, Perroud P-FO, Charron AJ, Mcdaniel SF, Khandelwal A, Quatrano RS (2009) Culturing the moss Physcomitrella patens. Cold Spring Harbor Protocols, 2009, pdb. prot5136.

Cronberg N, Natcheva R, Hedlund K (2006) Microarthropods mediate sperm transfer in mosses. Science, 313, 1255-1255.

Davey ML, Currah RS (2006) Interactions between mosses (Bryophyta) and fungi. Botany, 84, 1509-1519. 
Eppley S, Taylor P, Jesson L (2006) Self-fertilization in mosses: a comparison of heterozygote deficiency between species with combined versus separate sexes. Heredity, 98, 38-44.

Eppley SM, Taylor PT, Jesson LK (2007) Self-fertilization in mosses: a comparison of heterozygote deficiency between species with combined versus separate sexes. Heredity, 98, 38-44.

Fenster CB, Armbruster WS, Wilson P, Dudash MR, Thomson JD (2004) Pollination syndromes and floral specialization. Annual Review of Ecology Evolution and Systematics, 35, 375-403.

Geber MA, Dawson TE, Delph LF (eds) (1999) Gender and Sexual Dimorphism in Flowering Plants, Berlin Heidelberg, Springer-Verlag.

Gerson U (1969) Moss-Arthropod Associations. The Bryologist, 72, 495-500.

Gitelson A, Buschmann C, Lichtenthaler H (1999) The chlorophyll fluorescence ratio F735/F700 as an accurate measure of the chlorophyll content in plants. Remote sensing of environment, 69, 296-302.

Holsinger KE (2000) Reproductive systems and evolution in vascular plants. Proceedings of the National Academy of Sciences, 97, 7037-7042.

Jesson LK, Taylor P, Eppley SM (2007) Inbreeding depression in mosses occurs in a species with separate sexes but not in a species with combined sexes. American Journal of Botany, (in press).

Ligrone R, Duckett JG, Renzaglia KS (2012) Major transitions in the evolution of early land plants: a bryological perspective. Annals of Botany, 109, 851-871.

Lloyd D, Barrett SH, Harder L (1996) Pollen Dispersal and Mating Patterns in Animal-Pollinated Plants. In: Floral Biology. pp 140-190. Springer US.

Longton RE (1976) Reproductive biology and evolutionary potential in bryophytes. Journal of the Hattori Botanical Laboratory, 41, 205-223.

Longton RE (2006) Reproductive Ecology of Bryophytes: What Does It Tell Us about the Significance of Sexual Reproduction? Lindbergia, 31, 16-23.

Loveless MD, Hamrick JL (1984) Ecological determinants of genetic structure in plant populations. Annual review of ecology and systematics, 65-95.

Macfadyen A (1953) Notes on methods for the extraction of small soil arthropods. The Journal of Animal Acology, 65-77.

Marino P, Raguso R, Goffinet B (2009) The ecology and evolution of fly dispersed dung mosses (Family Splachnaceae): Manipulating insect behaviour through odour and visual cues. Symbiosis, 47, 61-76.

Mcdaniel SF, Atwood J, Burleigh JG (2013a) Recurrent evolution of dioecy in bryophytes. Evolution, 67, 567-572.

Mcdaniel SF, Neubig KM, Payton AC, Quatrano RS, Cove DJ (2013b) Recent gene capture on the UV sex chromosomes of the moss, Ceratodon purpureus. Evolution. 
Mcdaniel SF, Willis JH, Shaw AJ (2007) A linkage map reveals a complex basis for segregation distortion in an interpopulation cross in the moss Ceratodon purpureus. Genetics, 176, 2489-2500.

Muggoch H, Walton J (1942) On the dehiscence of the antheridium and the part played by surface tension in the dispersal of spermatocyctes in Bryophyta. Proceedings of the Royal Society B-Biological Sciences, 130, 448-461.

Paolillo DJJ (1981) The swimming sperms of land plants. BioScience, 31, 367373.

Perroud PF, Cove DJ, Quatrano RS, Mcdaniel SF (2011) An experimental method to facilitate the identification of hybrid sporophytes in the moss Physcomitrella patens using fluorescent tagged lines. New Phytologist, 191, 301-306.

Prigge MJ, Bezanilla M (2010) Evolutionary crossroads in developmental biology: Physcomitrella patens. Development, 137, 3535-3543.

Proctor MCF (2010) Recovery rates of chlorophyll-fluorescence parameters in desiccation-tolerant plants: fitted logistic curves as a versatile and robust source of comparative data. Plant Growth Regulation, 62, 233240.

Quatrano RS, Mcdaniel SF, Khandelwal A, Perroud PF, Cove DJ (2007) Physcomitrella patens: mosses enter the genomic age. Current Opinion in Plant Biology, 10, 182-189.

Rensing SA, Lang D, Zimmer AD et al. (2008) The Physcomitrella genome reveals evolutionary insights into the conquest of land by plants. Science, 319, 64-69.

Rosenstiel TN, Shortlidge EE, Melnychenko AN, Pankow JF, Eppley SM (2012) Sex-specific volatile compounds influence microarthropodmediated fertilization of moss. Nature, 489, 431-433.

SAS Institute (2012) JMP for Windows. Release 10.0.0. Cary, N.C.

Schaefer DG (2002) A new moss genetics: Targeted Mutagenesis in Physcomitrella patens. Annual Review of Plant Biology, 53, 477-501.

Shaw AJ (1991) The genetic structure of sporophytic and gametophytic populations of the moss, Funaria hygrometrica Hedw. Evolution, 45, 1260-1274.

Shaw AJ (2000) Population ecology, population genetics and microevolution. In: Bryophyte Biology. (eds Shaw AJ, Goffinet B) pp 369-402.

Cambridge, Cambridge University Press.

Srivastava DS, Kolasa J, Bengtsson J et al. (2004) Are natural microcosms useful model systems for ecology? Trends in Ecology \& Evolution, 19, 379-384.

Szövényi P, Ricca M, Shaw A (2009) Multiple paternity and sporophytic inbreeding depression in a dioicous moss species. Heredity, 103, 394403. 
Thompson JN, Cunningham BM (2002) Geographic structure and dynamics of coevolutionary selection. Nature, 417, 735-738.

Van Der Velde M, Bijlsma R (2000) Amount and structure of intra-and interspecific genetic variation in the moss genus Polytrichum. Heredity, 85, 328-337.

Van Der Velde M, During HJ, Van Der Zande L, Bijlsma R (2001) The reproductive biology of Polytrichum formosum: clonal structure and paternity revealed by microsatellites. Molecular Ecology, 10.

Vanderpoorten A, Goffinet B (2009) Introduction to Bryophytes, Cambridge, Cambridge Unversity Press.

Waite M, Sack L (2010) How does moss photosynthesis relate to leaf and canopy structure? Trait relationships for 10 Hawaiian species of contrasting light habitats. New Phytologist, 185, 156-172.

Walter DE (1987) Trophic behavior of" mycophagous" microarthropods. Ecology, 68, 226-229.

Waser NM, Chittka L, Price MV, Williams NM, Ollerton J (1996) Generalization in pollination systems, and why it matters. Ecology, 77, 1043-1060.

Wyatt R, Anderson LE (1984) Breeding systems in bryophytes. In: The Experimental Biology of Bryophytes. (eds Dyer AF, Duckett JG) pp 3964. London, Academic Press.

Yanoviak SP, Walker H, Nadkarni NM (2004) Arthropod assemblages in vegetative vs. humic portions of epiphyte mats in a neotropical cloud forest. Pedobiologia, 48, 51-58. 